\title{
Dynamics of Population Coding in the Cortex
}

\author{
Dissertation
}

zur Erlangung des Doktorgrades der Mathematisch-Naturwissenschaftlichen

Fakultäten der Georg-August-Universität zu Göttingen

\author{
vorgelegt von \\ Björn Naundorf \\ geboren in Offenbach am Main
}

Göttingen, 2005 
D7

Referent:

Koreferent:

Tag der mündlichen Prüfung:
Prof. Dr. Theo Geisel

Prof. Dr. Reiner Kree

28.6.2005 


\section{Contents}

$\begin{array}{lll}1 & \text { Introduction } & 7\end{array}$

2 Fundamentals 11

2.1 Single neurons as computational units $\ldots \ldots \ldots \ldots \ldots \ldots$

2.2 Action potentials as general communication paradigm in the cortex . . . . . . . . . . 12

2.3 Cortical "Noise": Ongoing activity in the cortex . . . . . . . . . . . . . 13

2.4 Intrinsic neuronal noise and the precision of single neurons . . . . . . . . . . . . . 14

2.5 Neural coding . . . . . . . . . . . . . . . . . . . . . . . 16

2.5.1 Population rate codes . . . . . . . . . . . . . . . . . . 16

2.5 .2 Spike-timing codes . . . . . . . . . . . . . . . . . 17

2.6 The Hodgkin-Huxley theory of AP initiation . . . . . . . . . . . . . . . . . 17

2.7 Reduced neuron models . . . . . . . . . . . . . . . . . . . . . . . . . 20

2.8 Modeling of cortical noise . . . . . . . . . . . . . . . . . . . . 21

2.8.1 From random synaptic inputs to Gaussian stochastic processes: The diffusion

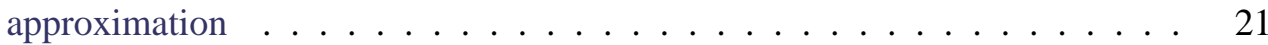

2.8.2 The effective leak approximation . . . . . . . . . . . . . 23

2.8 .3 Mean and variance modulation . . . . . . . . . . . . . . . . . . . . 24

2.9 Listening to the cortex: In vivo intracellular recordings . . . . . . . . . . . . . . 24

3 Dynamic response properties of populations of neuronal oscillators 25

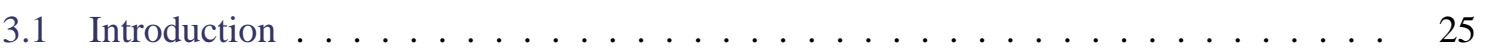


3.2 Studying population dynamics through noise driven neurons $\ldots \ldots \ldots$

3.2.1 The Silberberg-Experiment: Variance vs. mean coding . . . . . . . . . . . . 26

3.2.2 The Fellous-Experiment: Variance coding with fluctuating conductances . . 26

3.3 Theoretical predictions of dynamic neuronal responses $\ldots \ldots \ldots \ldots$

3.3.1 Ensemble dynamics and the linear response regime . . . . . . . . . . . . 28

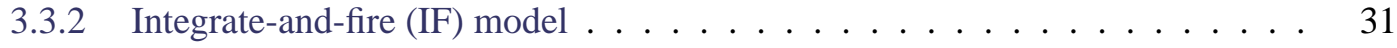

3.3.3 Summary of the predictions implied by the leaky IF model . . . . . . . . . . 31

3.3.4 Failure of IF models to describe the experimental results . . . . . . . . . . . 32

3.4 The generalized $\theta$-neuron: A generic phase oscillator model $\ldots \ldots \ldots$

3.4.1 From normal forms to phase oscillators . . . . . . . . . . . . . . . 33

3.4.2 The generalized $\theta$-neuron $\ldots \ldots \ldots \ldots \ldots \ldots \ldots \ldots \ldots$

3.5 Fokker-Planck equation for the dynamics of neuronal populations $\ldots \ldots$. . . . . 35

3.5.1 The limit of fast-decaying synaptic currents . . . . . . . . . . . . 36

3.5 .2 Time-dependent firing rate $\ldots \ldots \ldots \ldots \ldots \ldots \ldots$

3.5.3 Parameter choice . . . . . . . . . . . . . . . . . 38

3.6 Stationary response properties $\ldots \ldots \ldots \ldots \ldots \ldots \ldots$

3.6.1 Firing rate in the limit of fast-decaying synaptic currents . . . . . . . . . 40

3.6.2 Low-rate approximation . . . . . . . . . . . . . . . . . . . . . 40

3.6 .3 High-rate approximation . . . . . . . . . . . . . . . . . 42

3.6 .4 Medium-rate approximation . . . . . . . . . . . . . . . 42

3.6 .5 Coefficient of variation . . . . . . . . . . . . . . . . . . . 44

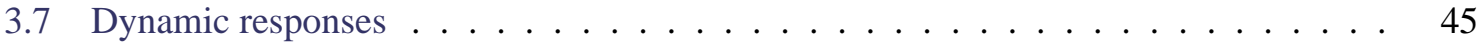

3.7 .1 General framework . . . . . . . . . . . . . . . . . . . . . 45

3.8 Universality of the high frequency behavior $\ldots \ldots \ldots \ldots$. . . . . . . . . . . . 47

3.8.1 Dynamics insensitive at $\mathrm{AP}\left(\theta_{s}=\pi\right) \ldots \ldots \ldots \ldots$. . . . . . . 47

3.8.2 The generic case $\left(\theta_{s} \neq \pi\right) \ldots \ldots \ldots \ldots \ldots$. . . . . . . . 47

3.9 A sparse matrix representation of the Fokker-Planck operator . . . . . . . . . . . . . 49

3.9.1 Eigenvalues and eigenfunctions for a correlated noise input . . . . . . . . . . 49

3.9.2 Properties of eigenvalues and eigenfunctions $\ldots \ldots \ldots$. . . . . . . 50 
3.9 .3 Matrix equation . . . . . . . . . . . . . . . . 50

3.9.4 Eigenvalues and eigenfunctions for a white noise input . . . . . . . . . . 53

3.9.5 Efficient computation of the stationary density and rate . . . . . . . . 54

3.10 Linear population response for arbitrary input frequencies . . . . . . . . . . . . 57

3.10.1 Dynamic responses for $\alpha=0$ to a current and noise modulation . . . . . . . 57

3.10 .2 Dynamic responses for $\alpha>0 \ldots \ldots \ldots \ldots$. . . . . . . . . . 61

3.10 .3 Nonlinear response for large stimulation amplitudes $\ldots \ldots$. . . . . . . . 65

3.11 Summary and discussion $\ldots \ldots \ldots \ldots \ldots \ldots$

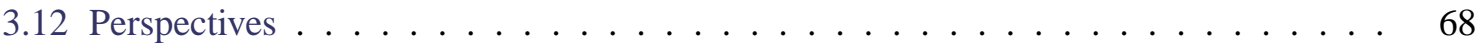

4 Anomalous action potential initiation in cortical neurons $\quad 69$

4.1 Action potentials: Energy budget considerations of the cortex . . . . . . . . . . . 69

4.2 Hodgkin-Huxley type models of cortical neurons . . . . . . . . . . . . . 70

4.3 Preprocessing of in vivo and in vitro recordings . . . . . . . . . . . . . . . 73

4.4 Phase-plane representation of action potential dynamics . . . . . . . . . . . . . . 75

4.4.1 Embedding of membrane potential recordings . . . . . . . . . . . . 75

4.5 AP initiation in cortical neurons $\ldots \ldots \ldots \ldots \ldots \ldots \ldots$

$4.5 .1 \quad$ Fast onset dynamics $\ldots \ldots \ldots \ldots \ldots \ldots$

4.5.2 Large variability . . . . . . . . . . . . . . . . . . . 79

4.6 Two antagonistic dynamical features in cortical AP initiation . . . . . . . . . . . . 79

4.7 Three hypotheses to explain the anomalous AP initiation in cortical neurons . . . . . 83

4.7.1 Ion channel stochasticity . . . . . . . . . . . . . . . . . 83

4.7.2 Where do APs initiate $\ldots \ldots \ldots \ldots \ldots$. . . . . . . . . . . 85

4.7.3 Sodium channel coupling at the node of Ranvier . . . . . . . . . . . . 88

4.7.4 Non-canonical sodium channel activation . . . . . . . . . . . . . . . . . . 91

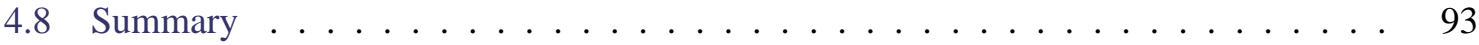

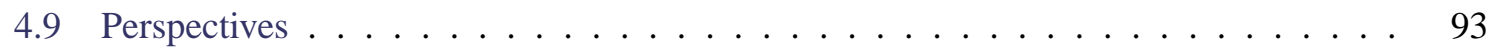

5 Functional consequences of anomalous action potential initiation: An exactly solvable model $\quad 95$ 


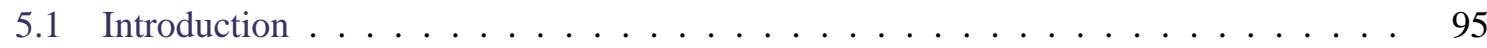

5.2 Subthreshold adaptation . . . . . . . . . . . . . . . . . . . . . . 96

5.3 Experimental evidence . . . . . . . . . . . . . . . . . . . . 96

5.4 The Gaussian neuron model with subthreshold adaptation $(V-\psi$ model $) \quad \ldots \ldots$

5.5 Spike-triggered average membrane potential . . . . . . . . . . . . . . . . 102

5.6 Dynamic response theory . . . . . . . . . . . . . . . . . . . . . . . . . 103

5.6.1 Response function for inputs with large amplitudes . . . . . . . . . . . 103

5.6 .2 Linear response theory . . . . . . . . . . . . . . . . . . . . . 105

$5.7 \quad V-\dot{V}$ correlation at action potential onset $\ldots \ldots \ldots \ldots$. . . . . . . . . . . . 109

5.7.1 An alternative mechanism to explain the AP onset variability in vivo . . . . . 109

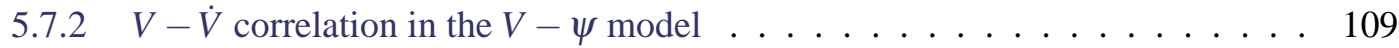

5.8 Gaussian neuron with a threshold in the $V-\dot{V}$ plane $\ldots \ldots \ldots$. . . . . . . . . . 109

5.9 Comparison with in vivo recordings . . . . . . . . . . . . . . . . . . . 112

5.9 .1 Methods ............................ 113

5.9.2 Results: AP initiation in vivo is not a threshold process in the $V-\dot{V}$ plane . . . 115

5.10 Summary and discussion $\ldots \ldots \ldots \ldots \ldots \ldots \ldots \ldots$

5.11 Perspectives . . . . . . . . . . . . . . . . . . . . . . . . 119

6 Summary and outlook 121

$\begin{array}{ll}\text { Bibliography } & 127\end{array}$

$\begin{array}{ll}\text { Acknowledgements } & 137\end{array}$ 


\section{Introduction}

In the nervous system, neurons communicate by sending and receiving action potentials (Bernstein, 1868; Huxley, 1963). Action potentials are actively generated pulses which are used for long-range communication as well as for communication within the cortex. They form the biophysical basis for all sensory processing and computation in the brain.

While the generation of action potentials on the cellular level is a well established process, it is yet unknown how the cortex combines them to form a neural code, which is used as a basis for communication and computation in the cortex.

Over the past decades, several experimental and theoretical studies fundamentally constrained the coding paradigms. One important constraint is imposed by the response properties of single cortical neurons. Recent measurements indicate that even in primary sensory areas many neurons respond to sensory stimuli with very low firing rates. It was reported, for example that in the somatosensory cortex, a single neuron typically emits action potentials at a firing rate substantially below $0.1 \mathrm{~Hz}$ (Margrie et al., 2002). For the auditory cortex it was even demonstrated, that neurons often emit one or no action potential per stimulus (DeWeese et al., 2003). These examples show that activity in the cortex is very sparse. Complementary theoretical studies corroborated these results, by quantifying the energy consumption of the cortex and comparing it with the energy required for neuronal signaling (Attwell \& Laughlin, 2001; Laughlin \& Sejnowski, 2003; Lennie, 2003). The authors of the studies concluded that in the human brain the energy supply of the cortex supports an average rate of less than one action potential per second and neuron.

Sparse coding on the single neuron level was demonstrated to work very well given that the timing of individual spikes is very precise (Diesmann et al., 1999). In in vivo recordings it was, however, demonstrated that the responses of the same neuron under repeated stimulation exhibit a large degree of variability both in their timing and in the number of evoked action potentials (Shadlen \& Newsome, 1998; Margrie et al., 2002; DeWeese et al., 2003).

On the other hand, it was demonstrated in a number of psychophysical experiments that cognitive processing of sensory stimuli can be achieved with a high accuracy on a time scale of approximately $150 \mathrm{~ms}$ for complex visual discrimination tasks (VanRullen \& Thorpe, 2001) and even less than $100 \mathrm{~ms}$ for auditory localization tasks (Populin \& Yin, 1998).

How can the cortex reliably process sensory information with millisecond precision, given that neurons respond very variable and often with less than one action potential per second? To answer this question it is instrumental to consider the functional organization of the cortex. Neurons in the cortex are densely packed and typically organized in a columnar architecture, where in each column, large numbers of neurons encode for similar stimulus attributes (It was for instance estimated that a hypercolumn in the visual cortex of a cat comprises more than $10^{5}$ neurons (O'Kusky \& Colonnier, 1982)). Thus, it may not suffice to study the stimulus encoding properties of single neurons, but one should rather consider populations of neurons (Hebb, 1949; Georgopoulos et al., 1986; Lee et al., 1988; Young \& Yamane, 1992; Fitzpatrick et al., 1997). What determines the coding properties of a 
population of neurons? Which cellular mechanisms of a single cell are important to understand the limiting factors of population coding? And more generally, what are the time-scales which determine the speed of processing in neuronal populations?

During recent years, researchers started to approach these questions experimentally. Because it is to date impossible to assess the coding properties of neuronal populations in the living brain, the typical experimental paradigm is to record intracellularly from single neurons in acute brain slices in vitro. The response of a neural population is in these types of experiments replaced with trial averaged responses. In in vivo recordings, the membrane potential trace of a cortical neuron is characterized by large fluctuations due to a large number of seemingly randomly incoming synaptic inputs, reflecting the ongoing spontaneous activity in the cortex. Thus, in the in vitro experiments, fluctuating currents are injected into the neuronal somata, whereby the statistics of the currents are chosen such that the resulting membrane potential fluctuations match the fluctuations observed in vivo (Destexhe \& Paré, 1999). This experimental paradigm allows to assess the population coding properties of a population of identical neurons in a very controlled fashion and realistic regime.

Using this recording technique, also the speed with which a population of neurons can respond to transient stimuli was assessed (Fellous et al., 2003; Silberberg et al., 2004). It is a common assumption that this speed is mainly determined by the stationary population firing rate or the membrane time constants of the single neurons which constitute the population. It thus came quite as a surprise that the experimentally measured response time can be much shorter than the integration time, when a stimulus paradigm is chosen in which information is encoded in the amplitude of the fluctuating input current.

To explain these counter-intuitive experimental results, I will develop in the first part of my thesis a theoretical framework to investigate the response of neuronal populations to time-dependent stimuli. To assess the response properties, I use a generic phase-oscillator model of a pyramidal neuron, the normal form of the dynamics of Type-I excitable membranes. Using the Fokker-Planck formalism, I assess the stationary, as well as the dynamical response properties in the presence of an in vivo like fluctuating synaptic input current.

The stationary response is characterized by an irregular spiking activity similar to what is observed in in vivo recordings of pyramidal neurons. To investigate the linear response properties, a novel approach is presented which is based on a sparse matrix representation of the Fokker-Planck operator. Using this approach, I derive the high frequency limit of the dynamic firing rate response. For the class of phase-oscillator models, the decay of the response amplitude turns out to be a power-law with an universal exponent, which is the same for conductance-based neuron models.

Furthermore, I show that the generic phase oscillator model can reproduces key aspects of the experimental results. As in the experiment, the speed with which the population can respond to timedependent stimuli is not limited by the population averaged stationary firing rate or the membrane time constant. Surprisingly, it crucially depends on the speed with which an action potential is dynamically initiated. For a very rapid initiation, a population can respond to frequencies up to $1 \mathrm{kHz}$, while a single neuron in the ensembles emits action potentials at a rate which is two orders of magnitude lower. Comparing the experimental results with the population response properties of the widely used class of integrate-and-fire models, it is revealed that this class of neuron models can in principle not reproduce the dynamics of real neurons in a realistic regime.

From the population response properties of the generic phase oscillator model, an important prediction is derived: To enable the cortex to rapidly process sensory stimuli, as observed in psychophysical ex- 
periments, cortical neurons should be specifically optimized to exhibit a fast action potential initiation dynamics.

To test this theoretical prediction and to elucidate the coding properties of cortical neurons in vivo, I analyze in chapter 4 the action potential initiating mechanism in intracellular recordings of cortical neurons in vivo and in vitro. It turns out that action potential initiation in these neurons is characterized by two key features: a very rapid initiation dynamics and a large variability of onset potentials. I then compare these dynamical features with the action potential initiation dynamics in recently proposed conductance-based neuron models. Unexpectedly, it turns out that the dynamical key features exhibited by cortical neurons are outside the range of behavior which can be described by the classical Hodgkin-Huxley theory. This suggests that a basic assumption of the theory is violated qualitatively, which has far reaching consequences for the modeling of dynamical neuronal responses.

While it is widely accepted that Hodgkin-Huxley type models do not well describe the dynamics of single voltage-gated ion channels, which are usually described by complex Markov models (Hille, 2001), it is commonly believed that on the cellular level they reproduce the mean field channel dynamics and the dynamics of action potentials. Thus, Hodgkin-Huxley type models are used to model the neuronal dynamics on all scales of cortical architecture, ranging from studies on spike timing (Schneidman et al., 1998) to large scale modeling of cortical networks (Hansel \& Sompolinsky, 1996).

The analysis of action potential initiation in cortical neurons further reveals a subthreshold adaptation mechanism. In chapter 5, I study the dynamical consequences of this mechanism in a new phenomenological model, the Gaussian neuron model, which includes the two key features of cortical action potential initiation in vivo. In contrast to conventional neuron models, in which the dynamics of the membrane potential is defined in terms of stochastic differential equations, the new model is based on the direct definition of a stochastic process with a given statistics. This alternative approach allows to give explicit analytic expressions for most stationary response properties and the dynamical population response to arbitrary time-dependent stimuli in a realistic regime. This specifically qualifies the model to be used in analytical studies on network dynamics, which often require explicit expressions for the rate transmission functions.

The further analysis of the dynamic response properties of the Gaussian neuron model, suggests that populations of cortical neurons are specifically optimized to respond with a high-pass filter characteristics. While the analysis of the generic phase-oscillator model revealed that the rapid onset dynamics leads to support of high-frequent stimuli in the population response, the subthreshold adaptation leads to a suppression of low frequent inputs.

The thesis is organized as follows. This introduction is followed by chapter 2 , in which I introduce fundamental concepts which are used throughout the thesis. In chapter $\mathbf{3}$, the theory of timedependent population coding in a generic neuron model, the generalized $\theta$-neuron, is developed. Using this model, an important prediction is derived: A direct dependence of the speed with which a population of neurons can represent time-varying signals on the action potential initiation dynamics. To elucidate the dynamical population response of populations of cortical neuron, I investigate in chapter 4 the action potential initiation in in vivo recordings of cortical pyramidal cells and show that key features of cortical AP initiation are not captured by the classical Hodgkin-Huxley theory. The functional consequences of cortical action potential initiation are further investigated in chapter 5, using a phenomenological neuron model which allows to calculate stationary and time-dependent response properties analytically. In chapter 6, I summarize the results obtained in this thesis and give a brief outlook on future research perspectives. 
1. Introduction 


\section{Fundamentals}

\subsection{Single neurons as computational units}

The concept that the brain is composed of single neurons which constitute independent units was not established until the beginning of the nineteenth century. Scientists of this period debated whether each neuron is a single entity or whether all neurons in the cortex were connected to form one enormous syncytium. Only by the developing new experimental techniques was it possible to resolve this issue. By using staining techniques, which made it possible to visualize single neurons, the famous histologist Ramon y Cajal (1906) persuaded the scientific community that each neuron is a closed unit connected through specialized contact points, which were called synapses.

Ultimately this issue was completely resolved when it became possible to visualize single synapses by using electron microscopy. At these connection points between neurons it was shown that there are two membranes, one belonging to the presynaptic neuron and the second belonging to the postsynaptic neuron, which are separated by a tiny interstitial space, the synaptic cleft (an exception constitute gap junctions, which are special direct connections between neurons).

Cortical neurons can be divided into several categories. The classic categorization is mainly based on the morphology of the cell body and the dendritic tree, there are, however, also other criteria which categorize neurons in the cortex with respect to the different areas they project to or with respect to the types of synaptic projections which can be excitatory or inhibitory. The most prominent cell type in the cortex are the pyramidal cells (Braitenberg \& Schüz, 1998). Their cell body has the shape of a pyramid with local dendrites extending from its base, and a long apical dendrite extending from the pyramid's apex towards the surface of the cortex, where it branches out extensively. Their main axons usually extend into the white matter and, in addition, a number of collateral axons make projections onto neighboring cells. Pyramidal cells are predominantly found in layers II/III and layer V/VI of the cortex. Due to their large dendritic trees they are believed to locally "integrate" the activity from their surrounding hypercolumnar network and send it through their axons in distant regions of the cortex. Recent studies revealed that the dendritic structure itself may take part in the computation that a single neuron performs (Poirazi et al., 2003; Polsky et al., 2004). In Fig. 4.8 on page 87, the morphology of reconstructed a layer $\mathrm{V}$ pyramidal cell is shown. Note extensive vertical axonal collaterals which go up to the cortical surface.

Physiologically, neurons can have two different effects on other cells. For a given neuron this effect is, with few exceptions, either excitatory or inhibitory (Dale's law). It was shown that pyramidal cells form exclusively excitatory synapses onto other efferent neurons, while another cell-class, the interneurons, form inhibitory synapses. Interneurons differ in their morphology from pyramidal cells. They are typically much smaller and usually make dendritic and axonal connections only locally in the cortex. Regarding their intrinsic dynamics, pyramidal cells differ substantially from interneurons (Tateno et al., 2004). As pyramidal neurons make up approximately $80 \%$ of all neurons in the cortex, I will focus in this thesis exclusively on pyramidal cortical neurons. 
Functionally, I've so far described the "effect" neurons can have onto one another. But how do neurons physically communicate with each other? In the next section I discuss the universal communication principle that is used in nervous system of all animals.

\subsection{Action potentials as general communication paradigm in the cortex}

The generation of action potentials (APs) is probably the most general principle nature invented for communication within the nervous system. APs are electric pulses which are actively generated by neurons and they are used both for short intra-cortical as well as long-range communication, where they can propagate unattenuated along axons for distances up to the meter range. APs can be found in practically all animals which exhibit a nervous system, ranging from invertebrates to humans.

The time-course of an AP was measured for the first time almost 150 years ago by the neurophysiologist Julius Bernstein (Bernstein, 1868), using a custom-made apparatus, the "differential rheotome". In his experiments, Bernstein stimulated an isolated frog nerve with a current pulse. At the opposite end of the nerve the change of the so-called injury current was measured via two electrodes positioned at the intact surface of the nerve and at the injured cross section. In this way, Bernstein measured what he called the "negative Schwankung des Nervenstroms" with sub-millisecond resolution. Given the experimental technology of the mid 19th century, the significance of these seminal experiments is tremendous and it took more than 80 years until Hodgkin and Huxley revealed the detailed biophysical theory of AP generation.

The Hodgkin-Huxley theory of the AP, developed during 1938-1952, is one of the great success stories of theoretical biology and one of the foundations of computational neuroscience. It is based on an intense collaboration between experiment and theory. In their experiments, in which they intracellularly measured the neuronal membrane potential (MP), they revealed that APs are actively generated pulses, during which the sign of MP can even reverse.

Moreover, Hodgkin and Huxley were able to dissect the ionic conductance involved in the generation of an AP, by using a so-called voltage-clamp protocol. They observed that step depolarization of a squid axon triggers an inward current followed by an outward current. Using ionic substitution, they demonstrated that this net current could be separated into two distinct components. The rapid inward current is carried by sodium ions, whereas the outward current is mainly carried by potassium ions. They further showed that these two currents result from independent permeability changes for sodium and potassium ions. At the time, this was a conceptional breakthrough, as voltage-gated ionic channels were not yet discovered.

Their most remarkable achievement, however, was the formulation of a quantitative model to describe the dynamics of the AP. This model could not only reproduce the AP shape in the squid axon but also the speed of AP propagation, the after-hyperpolarization of the membrane and sub-threshold MP oscillations. Today, the Hodgkin-Huxley theory of AP initiation is still the most biophysically realistic theory of AP initiation and a commonly used framework to describe the dependence of voltage-gated conductances beyond the AP. Hodgkin-Huxley type models are used to describe the MP dynamics in all kinds of neurons, ranging from the squid axon to different types of neurons in the neocortex. In Sec. 2.6, I will briefly describe the classical Hodgkin-Huxley theory of AP initiation. In Sec. 4.2, two recently proposed Hodgkin-Huxley type neuron models are studied, which were specifically extended to model the MP and spiking dynamics of cortical neurons. 


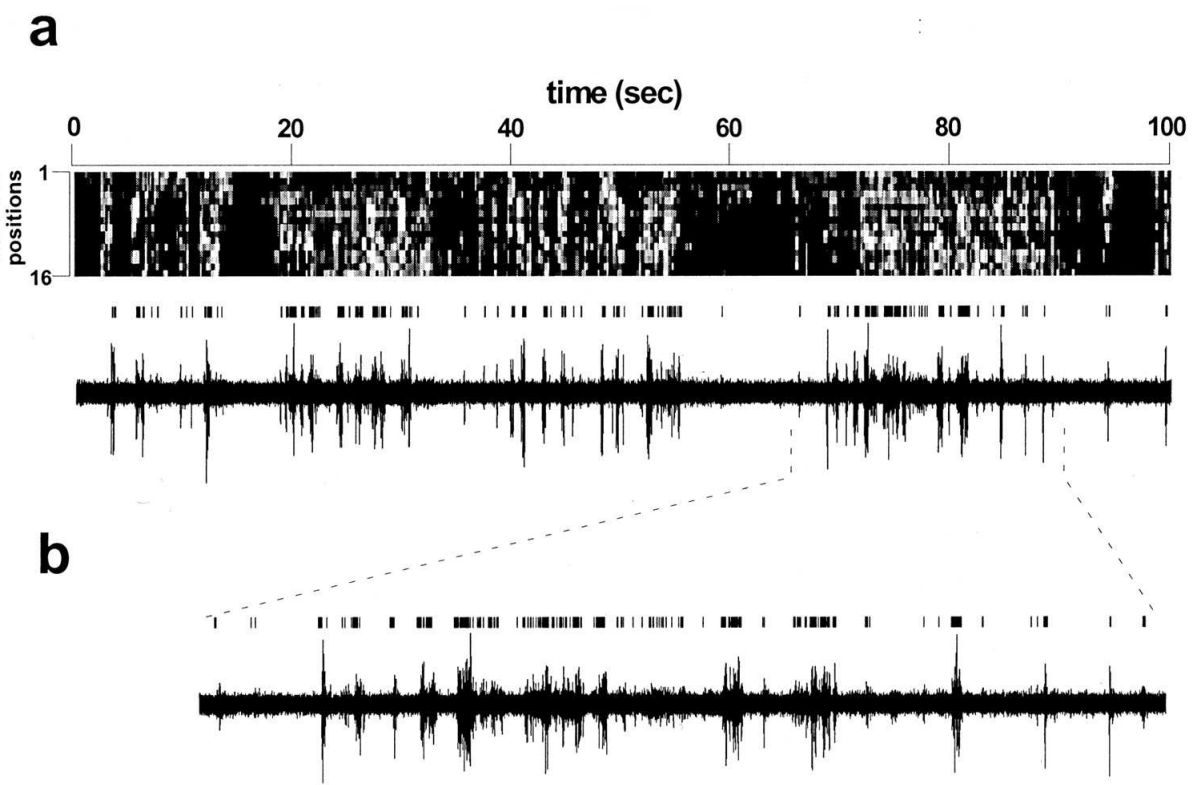

Figure 2.1: Spontaneous activity in the visual cortex of an awake behaving ferret. (a) Top trace: Time series graph was computed from a single $100 \mathrm{~s}$ acquisition trial for a P27 animal. Spike discharge rate at each electrode is encoded in gray scale along a different horizontal row (electrode 1 is the topmost row, and electrodes 2-16 are successive rows down). Bin width: $40 \mathrm{~ms}$. Bottom trace: Spike activity in electrode 10 for the same recording trial. (b) Close-up of the activity of electrode 10. The trace exhibits episodes of increased spontaneous activity. (Adapted from Chiu \& Weliky (2001))

From a theoretical point of view, the generation of APs provides a highly-nonlinear operation that fundamentally expands the information processing capabilites of a network of neurons. Imagine the extreme case, in which a neuron was a linear device. In this case, the dynamics of the intricate neural networks which constitute the cortex could be replaced by a single neuron with a complicated transmission function. Obviously, no complex computation could be realized in this case. It was first realized by McCulloch \& Pitts (1943), who studied a simple rate-based neuron model, that the inclusion of a non-linearity in the input-output relation of a neuron enables a nervous system to achieve any desired computation. They actually showed that a network of nonlinear model neurons is equivalent to a Turing machine and thus is able to perform any desired computation.

\subsection{Cortical "Noise": Ongoing activity in the cortex}

What is the ground state of the cortex in the absence of any sensory inputs and, more specifically, what is the dynamics of single neurons in this state? One might think, that, in particular in the primary sensory areas in the cortex, e.g. in the primary visual cortex, neuronal activity should either vanish or be very regular. Surprisingly, one finds a completely different behavior. In Fig. 2.1, the spontaneous spiking activity of many neurons recorded in parallel from the visual cortex of an awake ferret is shown. Each neuron emits spikes very irregularly with a rate of about $2 \mathrm{~Hz}$. During visual stimulation the firing rate increases, but nevertheless, the principle feature of temporally irregular spike emission 


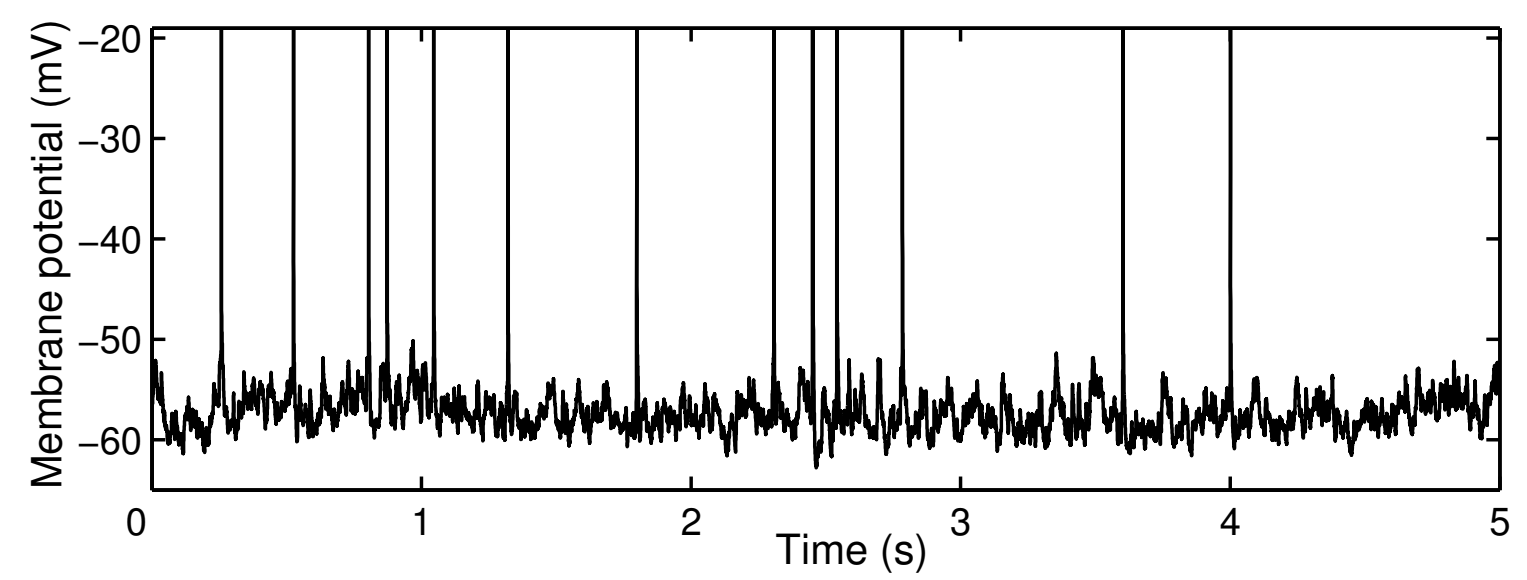

Figure 2.2: MP trace of a cortical neuron recording from the visual cortex of an anesthetized cat. The MP fluctuates due to ongoing synaptic bombardment. Even in the absence of a visual stimulus, the neuron emits APs irregularly at a rate of about $2 \mathrm{~Hz}$.

prevails. If this irregularity is analyzed statistically, it turns out that the spike train of a single neuron is almost indistinguishable from a random Poissonian process, which is completely determined by the mean number of emitted spikes per time interval (Softky \& Koch, 1993; Shadlen \& Newsome, 1998).

What is the impact of this spontaneous activity of the cortex on the MP dynamics of a single neuron? As I discussed above, each pyramidal neuron in the cortex receives inputs from about $10^{4}$ afferent neurons. If each afferent neuron exhibits a spontaneous rate of $1 \mathrm{~Hz}$, this input leads to an overall synaptic input with a frequency of about $10 \mathrm{kHz}$. In intracellular recordings of single neurons this "synaptic bombardment" results in a very variable time course of the MP (Fig. 2.2).

The functional role of these spontaneous subthreshold MP fluctuations was explored by numerous studies. It was argued that "synaptic noise" may enable single neurons to increase their discrimination properties to tuned inputs (Anderson et al., 2000; Volgushev \& Eysel, 2000) or enable a cortical network to dynamically change its sensitivity (Chance et al., 2002). Recent studies, in which the dynamics of an extended region of the visual cortex was recorded using optical imaging techniques, indicate that ongoing spontaneous activity may reflect a complex spatio-temporal state of the cortex which provides the context in which the brain evaluates sensory inputs (Arieli et al., 1996).

\subsection{Intrinsic neuronal noise and the precision of single neurons}

In the ground state of the cortex, single neurons exhibit a fluctuating MP and they spontaneously generate APs in an irregular fashion. Is this "noisy" dynamics only a consequence of the intricate cortical dynamics between neurons or is it also partially due to an intrinsically imprecise dynamics of single neurons? To answer this question, it is instrumental to assess the precision with which single neurons can respond, given that they receive the same stimulus repetitively. This single-neuron precision will then naturally serve as a lower bound for the precision of the cortical information processing.

Experimentally, the precision of single neuron dynamics was assessed in a seminal study by Mainen \& Sejnowski (1995). In this study, the authors injected two different types of current stimuli into isolated 

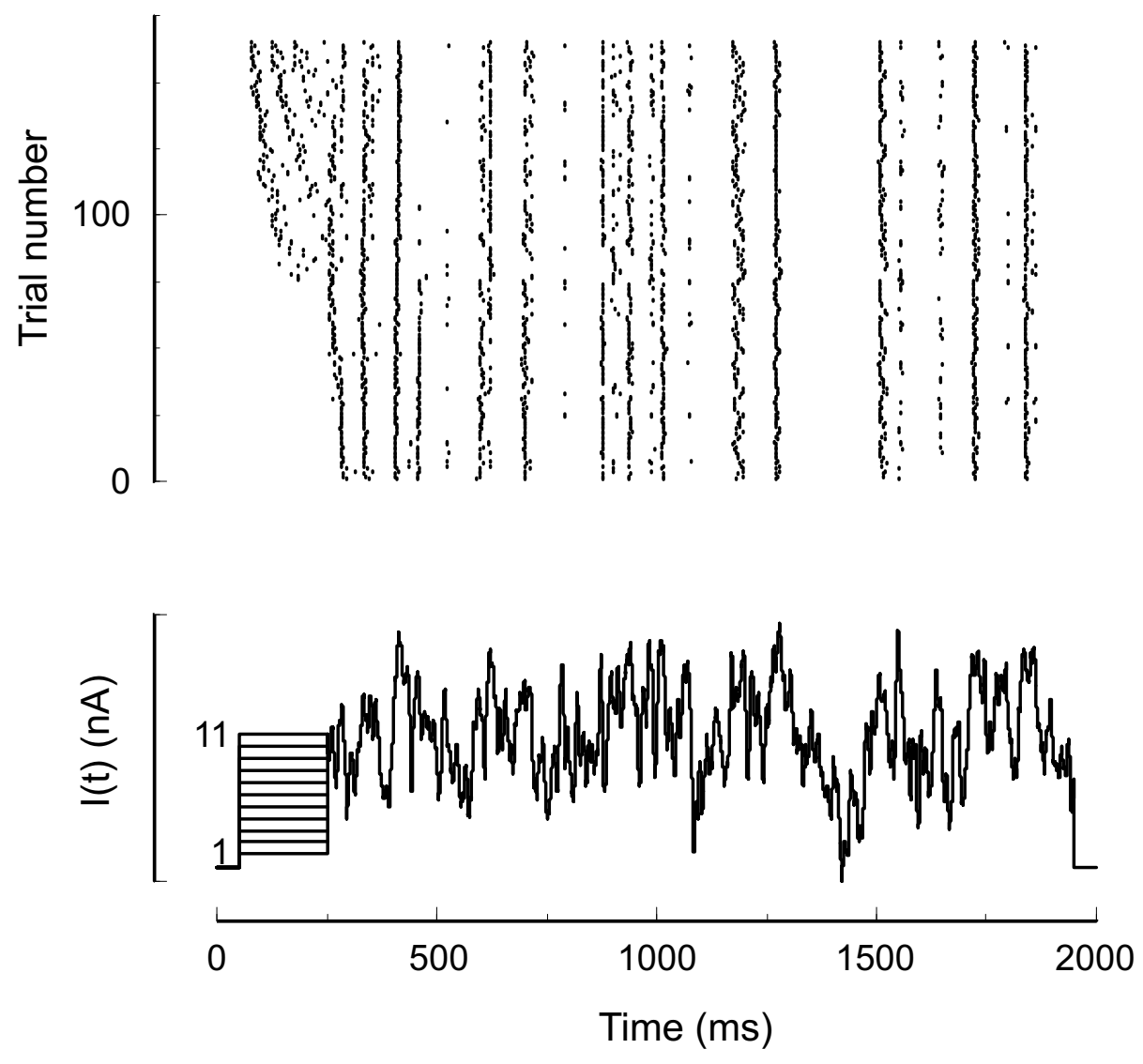

Figure 2.3: Precision of spike timing in a pyramidal neuron. Response of a pyramidal cell (top) to repetitive injection of a step and a subsequent noise current (bottom). Although the initial spike timings differ in response to the initial different step current, subsequent spikes lock onto the same spiking pattern with a high temporal precision, demonstrating that a single neuron is a precise dynamical system with low intrinsic noise. (Adapted from Tiesinga et al. (2002)) 
pyramidal cells in vitro. The first stimulus was a dc-current step and the second a "frozen" fluctuating noise current. When they injected a dc-current step, the trial-by-trial variability of the resulting spiketrain increased rapidly with an increasing length of the trial. When they, however, injected the same fluctuating input current repetitively, the trial-by-trial variability was very low (Fig. 2.3) and the neuron responded with millisecond precision. This response behavior was confirmed in an independent study with different types of input currents (Nowak et al., 1997).

Several following theoretical and experimental studies directly assessed the intrinsic noise sources in models of cortical neurons and their impact on the precision of single neuron coding. (Schneidman et al., 1998; White et al., 2000; Steinmetz et al., 2000; Jacobson et al., 2005). In these studies, two principle noise sources were studied: Channel noise, which is due to stochastic opening of a finite number of voltage-gated channels in a neuronal membrane, and thermal noise. They confirmed the experimental results, showing that the spike output of single neurons can be very precise under "in vivo-like" conditions and exhibit sub-millisecond spike-timing precision. The authors of these studies further showed that channel noise is the most limiting factor in the precision of single neurons. Compared to the size of the MP fluctuations induced by synaptic input, which typically has a standard deviation of $3-5 \mathrm{mV}$, the fluctuations induced by channel noise, however, were shown to be small, ranging from $0.15 \mathrm{mV}$ to $0.5 \mathrm{mV}$ in a pyramidal neuron (Jacobson et al., 2005).

\subsection{Neural coding}

When trying to answer the question, which code the cortex uses to process sensory stimuli and perform computations, it is crucial to keep in mind the large variability in the number and timing of APs in response to the presentation of identical sensory stimuli. There are basically to opposing views on the role of this irregularity and the underlying neuronal code. They are commonly referred to as "Spike timing codes" and "Population codes", and they represent two diametrically opposing views. The debate on the question which code is realized in the brain is still not settled, as enough decisive experimental data is not yet available. Accumulating evidence, however, suggests that both codes are used at the same time in different parts of the cortex.

\subsubsection{Population rate codes}

\subsubsection{Balance of excitation and inhibition}

It has been suggested that the irregular MP fluctuations and the resulting irregular spiking activity of a cortical neuron in vivo is due to balance between excitatory and inhibitory synaptic input, as proposed by Gerstein \& Mandelbrot (1964) and subsequently developed by Calvin \& Stevens $(1967,1968)$. In this setting, a single neuron produces only randomly timed spike patterns and information is encoded only in its mean firing rate. What type of cortical architecture would be required to support this coding scheme? As a single neuron in this scenario provides only a very poor estimate of the instantaneous spike rate, the cortex would have to use ensembles of neurons. Clustering of neurons with similar response properties is a well-established principle in primary sensory and motor areas of the cortex. Nearby neurons are often organized in columns, in which a large number of neurons encodes the same stimulus attributes. 


\subsubsection{Limits due to synchronous noise input}

How many neurons are needed to precisely encode an ensemble rate code? One might think that with an increasing number of neurons, the firing rate can be also estimated with increasing accuracy. This consideration, however, neglects the experimental finding, that neighboring neurons receive similar synaptic inputs and their firing times are thus slightly correlated with a correlation coefficient of roughly 0.12 (Riehle et al., 1997). Correlated noise that is shared by all members of a neuronal ensemble thus potentially places limits on the signaling power of population rate codes.

This issue was specifically addressed by Mazurek \& Shadlen (2002). They studied the population rate averaging and information transmission capabilities of neuronal populations with weakly correlated input spike trains across the population. One of their main conclusion was that the transmission of time-dependent signals is limited, for a realistic choice of parameters, to less than approximately $115 \mathrm{~Hz}$. This restriction could be an important bottleneck in information processing in the cortex. It is, however, an open issue what statistics the cortex uses to encode and decode information (Shamir \& Sompolinsky, 2004) or whether correlations might even be used to dynamically encode information (Riehle et al., 1997).

\subsubsection{Spike-timing codes}

The second, very appealing picture to explain the irregular dynamics of the cortex is that the exact timing of spikes is used to encode information. It was for instance demonstrated by the group of Abeles (Prut et al., 1998) that repeated patterns of spikes among ensembles of neurons can be found in prefrontal cortex. In visual cortex, synchronous spike patterns were observed in both anaesthesized and awake animals in a variety of conditions (Engel et al., 1999). In simulations of cortical neural networks it was demonstrated that under certain circumstances, embedded subnetworks can support the transmission of precisely timed spike-timing codes (Diesmann et al., 1999; Denker et al., 2004).

One has to keep in mind, however, that there is to date no evidence that the patterns, which are observed in experiments, constitute a neural code. It could be that they just occur at random due to the underlying cortical network. To test this assumption, it would be necessary to establish a connection between behavior and the observed temporal codes.

After these more general considerations on cortical dynamics, I will now in briefly discuss important theoretical models and concepts. I will first discuss the Hodgkin-Huxley theory, which will be very important for the derivation of the results in chapter 4. Afterwards, I will briefly discuss alternative simplified approaches to model neuronal dynamics and, in the end of the chapter, explain, how synaptic ongoing activity can be modeled and derive two important approximation, the diffusion and the effective leak approximation.

\subsection{The Hodgkin-Huxley theory of AP initiation}

The Hodgkin-Huxley theory of AP initiation describes the dynamics of the time-course of an AP by using a biophysical description of the underlying voltage-gated channels.

In a living neuron, as in most cells, a voltage drop across the membrane can be observed. This voltage drop, which is called resting potential, is due to different intra- and extracellular ionic concentrations. 
The potential difference induced by a single sort of ions is described by the Nernst equation (Nernst, 1888):

$$
E=\frac{k_{B} T}{z q} \ln \left(\frac{\text { [outside }]}{[\text { inside }]}\right)
$$

where $k_{B} T$ is thermal energy of an ion, $z q$ is charge carried by a single ion, and [outside] and [inside] denote the intra- and extracellular ionic concentrations. In the original Hodgkin-Huxley model, three types of ions are taken into account: Sodium, potassium and chloride (Hodgkin \& Huxley, 1952). In the following decades, the theory was adapted to include the dynamics of more types of ions, such as calcium ions.

The equilibrium potential for potassium ions $\left(E_{K}\right)$ in a living cell is usually around $-70 \mathrm{mV}$, the sodium potential $\left(E_{N a}\right)$ around $50 \mathrm{mV}$ and the leak potential $\left(E_{L}\right)$ which is due to chloride ions around $-60 \mathrm{mV}$, which is also close to the resting potential of many neurons.

Located in the neuronal membrane are so-called channels, which gate individual types of ions. The total current flowing across the membrane through all its ion channels is called the membrane current. It is determined by Kirchhoff's law, i.e. a sum of the contributions from the different type of channels:

$$
I_{M}=g_{L}\left(V-E_{L}\right)+g_{K}\left(V-E_{K}\right)+g_{N a}\left(V-E_{N a}\right),
$$

where $V$ denotes the MP and $g_{L}, g_{K}$ and $g_{N a}$ the conductances for chloride, potassium and sodium currents, respectively. Often, not the current and the conductances themselves are considered, but current and conductance densities.

The MP change resulting from a current flowing through the membrane is determined by the capacitance of the membrane $C_{m}$. It is related to the current by the following relation:

$$
C_{m} \frac{d V(t)}{d t}=I_{M}+I_{e}
$$

which is a dynamicalal equation for the MP of a neuronal membrane. Here, $I_{e}$ denotes an externally injected current.

The AP dynamics arises, because the sodium and potassium channel conductances $g_{K}(t)$ and $g_{N a}(t)$ are themselves time-dependent, whereas the specific leak conductance $g_{L}=0.3 \mathrm{mS} / \mathrm{cm}^{2}$ is assumed to be independent of the voltage. Hodgkin and Huxley assumed that several particles have to cooperatively come together to form an open channel through which an ionic current could flow. They modeled the probability for the formation of a potassium channel as:

$$
P_{K}(t)=n^{4}(t)
$$

Given that all channels in the membrane are independent, the total conductance for potassium channels is then given by:

$$
g_{K}(t)=\bar{g}_{K} P_{K}(t)=\bar{g}_{K} n^{4}(t),
$$

where $\bar{g}_{K}=36 \mathrm{mS} / \mathrm{cm}^{2}$ denotes the peak conductance of all potassium channels, given by the product of the single channel conductance and the number of channels in the membrane. Today, it is accepted that the voltage-gated potassium channel consists of a four-subunit structure (MacKinnon, 1991), which has to undergo a collective conformational change to open the channel.

The transitions between the open and closed state of each subunit are assumed to occur with voltage- 
dependent rates $\alpha_{n}(V)$ and $\beta_{n}(V)$. They are described by the following first-order differential equation:

$$
\frac{d n(t)}{d t}=\alpha_{n}(V)(1-n(t))-\beta_{n}(V) n(t),
$$

where the first term describes the opening process and the second term the closing process. Conventionally, this equation is brought into a more compact form:

$$
\tau_{n} \frac{d n(t)}{d t}=n_{\infty}(V)-n(t)
$$

with $n_{\infty}(V)=\alpha_{n}(V) /\left(\alpha_{n}(V)+\beta_{n}(V)\right)$ and $\tau_{n}=\left(\alpha_{n}(V)+\beta_{n}(V)\right)^{-1}$. The opening and closing rates were fitted by Hodgkin and Huxley to the following functions:

$$
\alpha_{n}(V)=\frac{10^{-2}(V+55)}{1-\exp (-0.1(V+55))}, \quad \beta_{n}(V)=\exp (-(V+65) / 80) / 8 .
$$

In contrast to the potassium channel, the sodium channel opens only transiently. The probability that it is open is written in the form $m(t)^{k}$, where $m(t)$ is an activation variable similar to $n$. The exponent $k$ is an integer, which was estimated by Hodgkin and Huxley as 3. To describe the transient opening of the channel, Hodgkin and Huxley used a second gate $h$, which is called the inactivation variable. The probability of a sodium channel to be open is thus given by:

$$
P_{N a}(t)=m(t)^{3} h(t),
$$

where the dynamics of $m(t)$ and $h(t)$ are given by:

$$
\begin{aligned}
\tau_{m} \frac{d m(t)}{d t} & =m_{\infty}(V)-m(t) \\
\tau_{n} \frac{d h(t)}{d t} & =h_{\infty}(V)-h(t)
\end{aligned}
$$

The functions $m_{\infty}(V), h_{\infty}(V), \tau_{m}(V)$ and $\tau_{h}(V)$ are defined as for the opening gate of the potassium channel. The opening and closing rates were fitted by:

$$
\begin{array}{cl}
\alpha_{m}(V)=\frac{V+40}{10(1-\exp ((V+40) / 10))} & \beta_{m}=4 \exp (-(V+65) / 18) \\
\alpha_{h}(V)=\frac{V+40}{100 / 7(1-\exp ((V+65) / 20))} & \beta_{h}=1 /(1+\exp (-(V+35) / 10)
\end{array}
$$

The sodium conductance is then defined as:

$$
g_{N a}(t)=\bar{g}_{N a} P_{N a}(t)
$$

with the sodium peak conductance $\bar{g}_{N a}=120 \mathrm{mS} / \mathrm{cm}^{2}$.

In Fig. 2.4, a measured AP of the squid axon is compared with a simulation of the dynamics of the Hodgkin-Huxley model. At the beginning of an AP, sodium channels open which leads to a rapid depolarization of the membrane. The delayed inactivation of sodium channels and opening of potassium channels results in the subsequent downstroke of the AP and a characterstic after-hyperpolarization.

The Hodgkin-Huxley equations were extended in many ways to describe the dynamics of different 

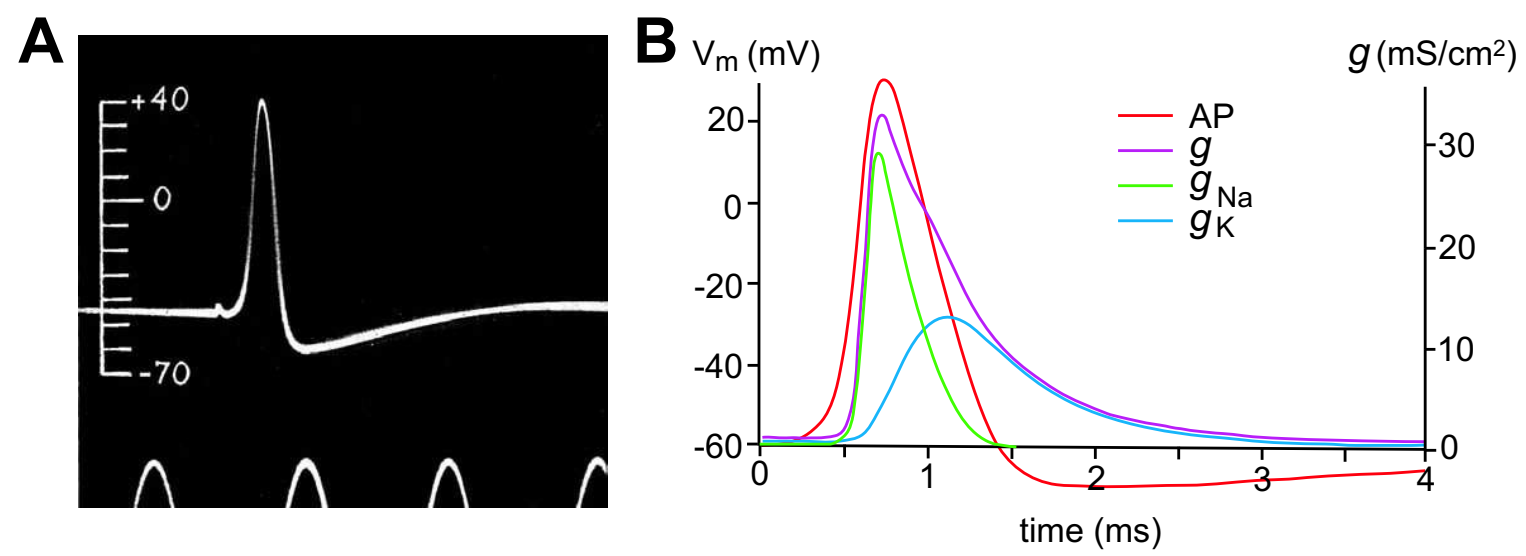

Figure 2.4: Comparison between An AP from the squid giant axon. (A) The first intracellular recording of an AP from the squid axon. (B) Simulated MP $V_{m}$ and ionic conductances according to Eqs. $(2.3,2.5,2.14)$. (Adapted from Hodgkin \& Huxley (1952))

types of neurons, thereby creating a whole class of models, to which I will in the following refer to as Hodgkin-Huxley type models. These models can include more types of channels (e.g. calcium channels) or model large, spatially extended neurons, taking into account many different compartments (in chapter 4, I study two examples of Hodgkin-Huxley type models which were specifically adapted to model the dynamics of cortical pyramidal neurons.

\subsection{Reduced neuron models}

The Hodgkin-Huxley model equations define a three-dimensional dynamical system, which cannot be solved analytically and which is hard to analyze. This turns out to be even more the case if one considers Hodgkin-Huxley type models of cortical neurons, which typically incorporate more than six dynamical degrees of freedom. Often, however, the exact form of an AP is not important and the dynamics can be reduced by e.g. adiabatically eliminating the dynamics of the fast sodium activation. In this spirit, FitzHugh (Fitzhugh, 1960) and subsequently Morris and Lecar (Morris \& Lecar, 1981) derived two-dimensional neuron models.

In the language of dynamical systems theory it was then possible to study the dynamics of these models using phase-plane analysis in terms of stable or unstable fixed points, nullclines, invariant manifolds and by using bifurcation theory (Rinzel \& Ermentrout, 1998).

Probably the most consequent reduction was proposed by Ermentrout \& Kopell (1986). They noticed that in a large sub-class of conductance-based neuron models, the dynamics undergoes a co-dimension one saddle-node bifurcation when the model is brought to the repetitive firing regime. Close to this bifurcation, the dynamics is restricted to a one-dimensional sub-manifold (Strogatz, 2001), the normal form of the bifurcation. They then mapped the resulting dynamics onto a phase oscillator, defining the $\theta$-neuron model. The dynamical properties of this model will be studied in the following chapter to determine its population coding properties.

Besides the neuron models, which can be derived from more complex conductance-based models, there is also the large class of phenomenological models. The most popular of them is the class of 
integrate-and-fire models (Lapicque, 1907; Abbott, 1999), which I will describe in the following chapter. This class of models can be seen as the workhorse of theoretical neuroscience. Because of its simplicity it was used to theoretically study various aspects of neuronal dynamics, ranging from studies on synfire chains (Diesmann et al., 1999), neural networks (Tsodyks \& Sejnowski, 1995; van Vreeswijk \& Sompolinsky, 1996; Brunel \& Hakim, 1999) to dynamical signal processing (Knight, 1972; Lindner \& Schimansky-Geier, 2001; Brunel et al., 2001) in neuronal populations (For a review see (Abbott, 1999)). One has to keep in mind, however, that this class of models is defined purely phenomenologically and thus its range of validity is not a-priori clear.

\subsection{Modeling of cortical noise}

Central to the modeling of ongoing activity in the cortex is the theory of stochastic processes. This theory provides a number of useful tools that I will employ throughout my thesis. For a general introduction see (Stratonovich, 1963; Risken, 1996; Gardiner, 2004).

In the remainder of this chapter, I will give an explicit example, how the effect of a large number of synaptic inputs on the MP of a single neuron can be modeled using the theory of stochastic processes. In particular, I will explain two important approximation, which will be used throughout this thesis: The diffusion approximation and the effective leak approximation.

\subsubsection{From random synaptic inputs to Gaussian stochastic processes: The diffusion approximation}

Different approaches were proposed to model the MP fluctuations which are exhibited by recordings of cortical neurons in vivo. Detailed biophysical models explicitely considered thousands of synapses (Tiesinga et al., 2000; Tuckwell et al., 2002) in single compartmental neurons or even in models which incorporated dendritic morphology. A complementary approach is based on modeling synaptic noise as random processes. In this class of models, it is assumed that the large number of synaptic inputs, which impinge onto a single neuron in parallel, result in an effective stochastic process with certain statistical properties. In this section I will briefly sketch, how the statistical random process can be derived from a model in which a large number of excitatory and inhibitory synapses open at random times, leading to stochastic fluctuations of the conductance of a neuronal membrane and resulting in a fluctuating current across the membrane.

Assume that synaptic inputs impinge onto the neuronal membrane at times $\left\{t_{i}\right\}$, where each synaptic event changes the conductance of the membrane by an amount $g_{\max }$ and decays with a rate $\beta$ :

$$
\frac{d g(t)}{d t}=-\beta g(t)+g_{\max } \sum_{i} \delta\left(t-t_{i}\right)
$$

The solution of this differential equation is given by:

$$
g(t)=g_{\max } \sum_{i} \Theta\left(t-t_{i}\right) e^{-\beta\left(t-t_{i}\right)}
$$

Given that the times $\left\{t_{i}\right\}$ are uncorrelated, the power spectral density (Stratonovich, 1963) of the 
A

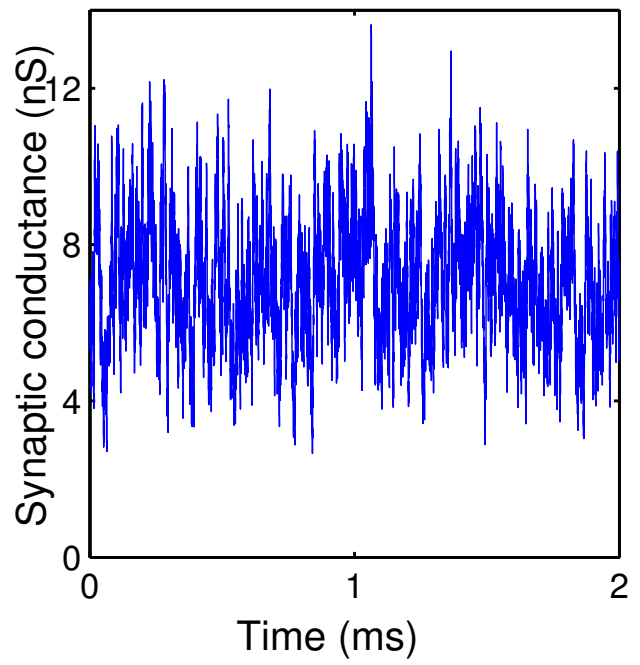

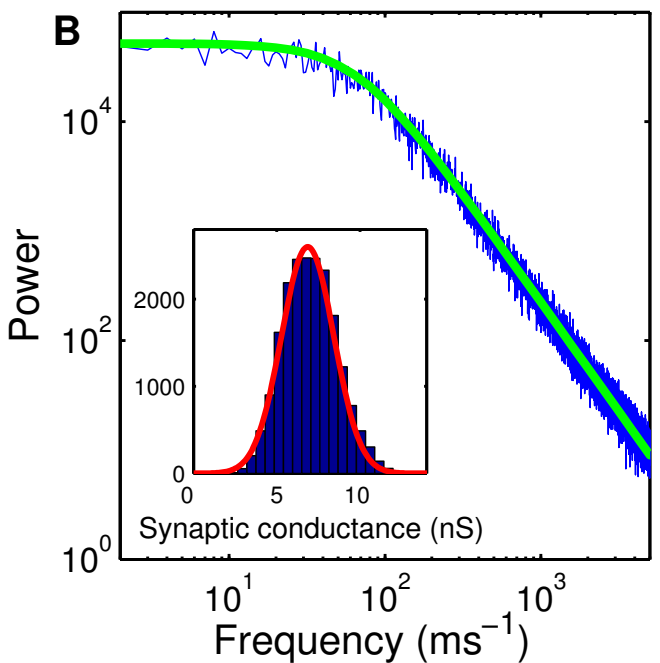

Figure 2.5: The net-effect of a large number of random synaptic inputs. (A) Synaptic conductance resulting from 1000 independent synapses. $\left(\beta=0.21 \mathrm{~ms}^{-1}, g_{\max }=0.72 \mathrm{nS}\right)$ Presynaptic inputs of each synapse are Poisson distributed with an average rate of $\lambda=2 \mathrm{~Hz}$. The conductance trace exhibits an irregular dynamics, resulting from the random timing of presynaptic inputs. (B) Power spectrum of the conductance. It was calculated from the numerical time course in (A). The solid green line is the prediction from Eq. (2.17). Both curves coincide. Inset: Histogram of conductances in (A). The histogram is well fit by a Gaussian with mean and variance given by Eq. (2.18).

conductance $g(t)$ is given by:

$$
P_{g-\langle g\rangle}(\omega)=\frac{g_{\max }^{2} \lambda}{\beta^{2}+\omega^{2}}
$$

where the $\lambda$ denotes the presynaptic rate. The mean open probability and its standard deviation are given by:

$$
\langle g\rangle=\lambda g_{\max } / \beta, \quad \sigma_{g}^{2}=(2 \pi)^{-1} \int P_{g-\langle g\rangle}(\omega) d \omega=\frac{\lambda g_{\max }^{2}}{2 \beta}
$$

It can be further shown that all higher moments do not exist. An equivalent process is given by an Ornstein-Uhlenbeck process (Uhlenbeck \& Ornstein, 1930):

$$
\frac{d g(t)}{d t}=\tau^{-1}(\langle g\rangle-g)+\sqrt{2 \tau^{-1}} \sigma_{g}^{2} \xi(t),
$$

with $\tau=\beta^{-1}$. In Fig. 2.5, a direct simulation of Eq. (2.15) incorporating random synaptic events $\left\{t_{i}\right\}$ is compared with the equivalent Ornstein-Uhlenbeck process. The conductance fluctuates randomly and the distribution of conductances, as well as the power spectral density coincides well with the Ornstein-Uhlenbeck process, demonstrating that the net-effect of a large number of synaptic inputs is effectively equivalent to a simple random process.

Remark: In the derivation I assumed that individual synaptic events are statistically independent. Strictly, this is not true for neurons in the cortex. As shown by Riehle et al. (1997); Shadlen \& Newsome (1998), there may be significant correlations between spike-trains of different cortical neurons. The role of these correlations with respect to computation in the cortex is currently 
unknown. In principle, synchronized input can be incorporated in the above derivation, leading to different expressions for the mean conductance $\langle g\rangle$ and the variance $\sigma_{g}^{2}$. Generally, an increase in synchrony will increase the variability of the conductance fluctuations.

\subsubsection{The effective leak approximation}

So far, I only considered the case of one type of synapses, either excitatory or inhibitory. With a combination of both the total current across the membrane is given by:

$$
I_{\mathrm{syn}}=I^{(e)}+I^{(i)}=g^{(e)}(t)\left(V^{(e)}-V\right)+g^{(i)}(t)\left(V^{(i)}-V\right),
$$

where $g^{(e)}(t)$ is the conductance due to excitatory conductances, $g^{(i)}(t)$ the conductance due to inhibitory synapse and $V^{(e)}$ and $V^{(i)}$ their corresponding reversal potentials.

It was shown by Bernander et al. (1991); Burkitt (2001); Brunel \& Wang (2001); Rudolph \& Destexhe (2003) that if (i) the total membrane conductance compared to the conductance of a single synapse is small and (ii) the voltage deflections due to isolated inputs are small compared to the distance to the reversal potential, an effective leak approximation can be applied.

The dynamics of the MP in a one-compartmental cortical neuron is in general given by:

$$
C_{m} \frac{d V}{d t}=g^{\text {leak }}\left(V^{\text {leak }}-V\right)+\phi(V)+\sum_{j \in\{e, i\}} g^{(j)}(t)\left(V-V^{(j)}\right)
$$

Here, $C_{m}$ denotes the capacity of the membrane and $g^{\text {leak }}$ and $V^{\text {leak }}$ is the leak conductance and reversal potential, respectively. The function $\phi(V)$ includes all nonlinearities due to active, voltage-dependent channels. Introducing the mean MP $\bar{V}$ and the total conductance $G(t)=g^{\text {leak }}+\sum_{j \in\{e, i\}} g^{(j)}(t)$, Eq. (2.21) can be written as:

$$
C \frac{d V}{d t}=G(t)(\bar{V}-V)+\phi(V)+\left(\bar{V}-V^{(e)}\right) g^{(e)}(t)+\left(\bar{V}-V^{(i)}\right) g^{(i)}(t)
$$

Replacing now the time-dependent total conductance $G(t)$ by its mean value $\bar{G}(t)$ yields:

$$
C \frac{d V}{d t}=\bar{G}(\bar{V}-V)+\phi(V)+I_{\text {syn }}(t),
$$

where the conductance input is replaced by a fluctuating current $I_{\text {syn }}(t)$ given by,

$$
I_{\text {syn }}(t)=\left(\bar{V}-V^{(e)}\right) g^{(e)}(t)+\left(\bar{V}-V^{(i)}\right) g^{(i)}(t),
$$

which is independent of the MP $V(t)$. 


\subsubsection{Mean and variance modulation}

The mean and variance of the synaptic input $I_{\mathrm{syn}}(t)$ can be derived from the mean and variance of the time-dependent synaptic conductances:

$$
\begin{aligned}
\left\langle I_{\mathrm{syn}}\right\rangle & =\left(\bar{V}-V^{(e)}\right)\left\langle g^{(e)}\right\rangle+\left(\bar{V}-V^{(i)}\right)\left\langle g^{(i)}\right\rangle \\
\sigma_{I_{\mathrm{syn}}}^{2} & =\left(\bar{V}-V^{(e)}\right)^{2} \sigma_{g^{(e)}}^{2}+\left(\bar{V}-V^{(i)}\right)^{2} \sigma_{g^{(i)}}^{2}
\end{aligned}
$$

If the synaptic time constants are assumed to be the same, this process can be synthesized using an Ornstein-Uhlenbeck process, as described for the time-dependent conductance fluctuations in the previous section.

Given that the synaptic time constants are fixed, the two statistical quantities which can change as a function of time are the mean current $\left\langle I_{\mathrm{syn}}\right\rangle$ and the variance of the current $\sigma_{I_{\mathrm{syn}}}^{2}$. Experimentally, it was demonstrated that the two quantities are indeed two different channels for information coding. In intracellular recordings of orientation selective neurons from cat visual cortex it was shown that stimuli which lead to large differences in the mean MP of a neuron and in the firing rate, the amplitude of the subthreshold fluctuation remained approximately constant (Anderson et al., 2000; Volgushev \& Eysel, 2000). This was even true, when the contrast of the input was changed, resulting in a change in the firing rate of the neuron by a factor of two. Network studies, on the other hand, suggest that in a balanced state of excitatory and inhibitory synaptic inputs, a modulation of the variance of the fluctuations can support a very rapid coding (Tsodyks \& Sejnowski, 1995; van Vreeswijk \& Sompolinsky, 1996, 1998).

\subsection{Listening to the cortex: In vivo intracellular recordings}

In chapter 4 and 5 of my thesis, I compare theoretical models with in vivo intracellular recordings of cortical neurons. This recording technique is very demanding and one of the pillars of experimental neuroscience. In in vivo intracellular recordings, a sharp or patch pipette (a small glass electrode) is inserted directly into the cortex and into the soma of a neuron of an anesthetized (Creutzfeldt, 1983; Pei et al., 1991) or even awake animal (Margrie et al., 2002).

Compared to in vitro recordings in acute slices, in which a neuron is not exposed to network activity, in vivo recordings allow to measure the dynamical properties of cortical neurons in their normal surrounding. In vitro recordings, on the other hand, allow to measure the response properties of single neurons in a much more controlled fashion.

I will not present here the detailed and intricate recording procedure that is used to measure the MP of cortical neurons in vivo. It can be found for example in (Volgushev et al., 2002). 


\section{Dynamic response properties of populations of neuronal oscillators}

\subsection{Introduction}

How fast can a population of cortical neurons transmit time-varying stimuli in vivo? In this chapter I first describe two recent experimental studies which assessed this question. These studies showed that a coding scheme, which modulates the variance of the synaptic input fluctuations, surprisingly leads to faithful transmissions of input currents which are modulated on a much shorter time-scale than the neuronal membrane time constant or the time constant induced by the inverse stationary firing rate. I then discuss several theoretical studies which preceeded these experiments and partially motivated them. In these studies, the dynamic response properties of the class of integrate-and-fire models was assessed. I will show, however, that this model class, although widely used for theoretical studies on neural dynamics, is unable to reproduce the features of the dynamic response properties of real neurons which were observed in the experiments.

To explain the experimental results, I develop in the following a theoretical framework to investigate the stationary and dynamic population response properties of a generic neuronal oscillator model, a generalization of the normal form dynamics exhibited by Type-I excitable neuronal membranes. This model incorporates an important dynamical feature which is missing in integrate-and-fire models: A realistic AP initiation dynamics. The stationary response properties are calculated analytically in the limit of vanishing synaptic time constants, i.e. a temporally uncorrelated fluctuating input current. For the more realistic case of finite synaptic time constants, in which the input current is temporally correlated, approximate solutions for the stationary firing rate are derived. I then focus on the dynamic response properties in the linear response regime and show that, irrespective of model details, the transmission functions in the high-frequency limit always decay as a power law. Surprisingly, the power-law exponent qualitatively depends on the phase $\theta_{s}$ of the oscillator at which a spike is emitted: If at $\theta_{s}$ the dynamics is insensitive to external inputs, transmission functions decay as (i) $\omega^{-3}$ for the case of a modulation of a temporally uncorrelated input and (ii) as $\omega^{-2}$ for a modulation of the mean input current in the presence of a correlated and uncorrelated noise and in the case of a modulation of the amplitude of a temporally correlated noise input. If the insensitivity condition is lifted, all transmission functions decay as $\omega^{-1}$, which is the behavior reported for conductance-based neuron models.

To compute the dynamical transmission function for arbitrary frequencies, I then introduce a novel sparse matrix representation of the Fokker-Planck operator, which describes the dynamics of the population response. Using this matrix representation, the full transmission function for arbitrary stimulation frequencies is computed. It turns out that the cut-off frequency, up to which transient stimuli can be transmitted unattenuated, crucially depends on the AP onset dynamics. When the AP onset dynamics is very rapid, the decay of the transmission function can be shifted to very large frequencies up to $1 \mathrm{kHz}$. Furthermore, the transmission amplitude for modulations of the amplitude of the 
synaptic input current is typically much larger at high frequencies than for a modulation in the mean synaptic current.

In the last section, I will briefly discuss the response to large input stimuli and argue that for typical model parameters the response properties are well captured by the linear response theory.

\subsection{Studying population dynamics through noise driven neurons}

So far, there have been few experimental attempts to assess the information transmission capabilities of cortical neurons in the presence of fluctuating synaptic inputs (Fellous et al., 2003; Silberberg et al., 2004). As it is to date not possible to assess the population coding properties of cortical neurons in vivo, the two experimental studies, which I will briefly sketch in the following, were performed in vitro. As discussed in the previous chapter, cortical neurons in vivo exhibit a continuous bombardment of synaptic inputs, leading to a fluctuating MP. To model this in vivo regime, fluctuating input currents were injected into the somata of the neurons. The statistically properties of these input currents were adjusted such that the statistics of the MP fluctuations matched the statistics of the fluctuations exhibited by in vivo intracellular recordings of cortical neurons. The population response properties were then assessed by calculating trial averages, where different realizations of the fluctuations, but the same stimulus was injected repetitively.

\subsubsection{The Silberberg-Experiment: Variance vs. mean coding}

Is there a difference in the response speed to stimuli which are encoded in the mean input current compared to stimuli which modulate the amplitude of the fluctuating input current? This question was assessed in a study by Silberberg et al. (2004). The surprising outcome of this study was that if the amplitude of the fluctuating input current was changed in a step-like fashion, the average output rate could adapt almost instantaneously, on a time-scale of $1 \mathrm{~ms}$ (Fig. 3.1B). On the other hand, a step change in the mean input current resulted only in a slow response, with a time-constant of approximately $20 \mathrm{~ms}$ (Fig. 3.1A).

The study was performed in two steps. First, realistic synaptic fluctuating currents from whole cell voltage-clamp recordings were obtained with different levels of excitation in vivo. These currents were subsequently injected into the somata of pyramidal neurons in vitro. At a given time, either the mean input current or the variance of the fluctuations was changed abruptly. The amplitude of the change was adjusted such that the stationary rate after the step was the same under both conditions.

\subsubsection{The Fellous-Experiment: Variance coding with fluctuating conductances}

The speed with which a population of neurons can respond to a change in the variance of a fluctuating input was also assessed in a second study (Fellous et al., 2003). In this study, it was shown, in accordance with the first study, that the response to modulations in the variance of the fluctuations can be very fast, on a time scale of less than $10 \mathrm{~ms}$. Moreover, the authors of this study assessed the detection probability of transient stimuli, which they defined as the fraction of the number of APs which where locked to the stimulus, relative to the overall number of generated APs in a trial. 


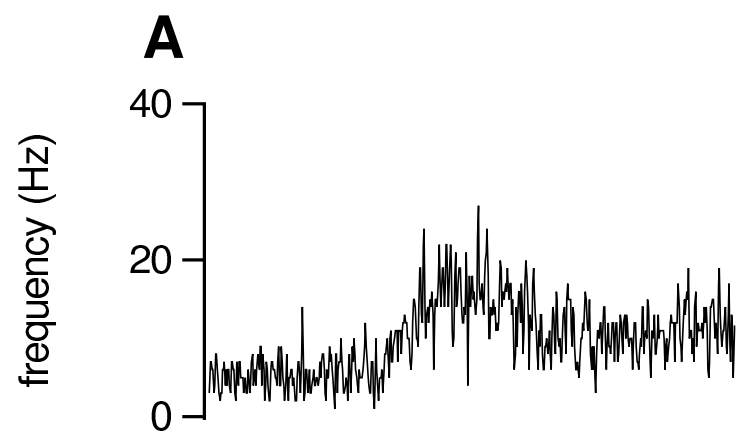

B
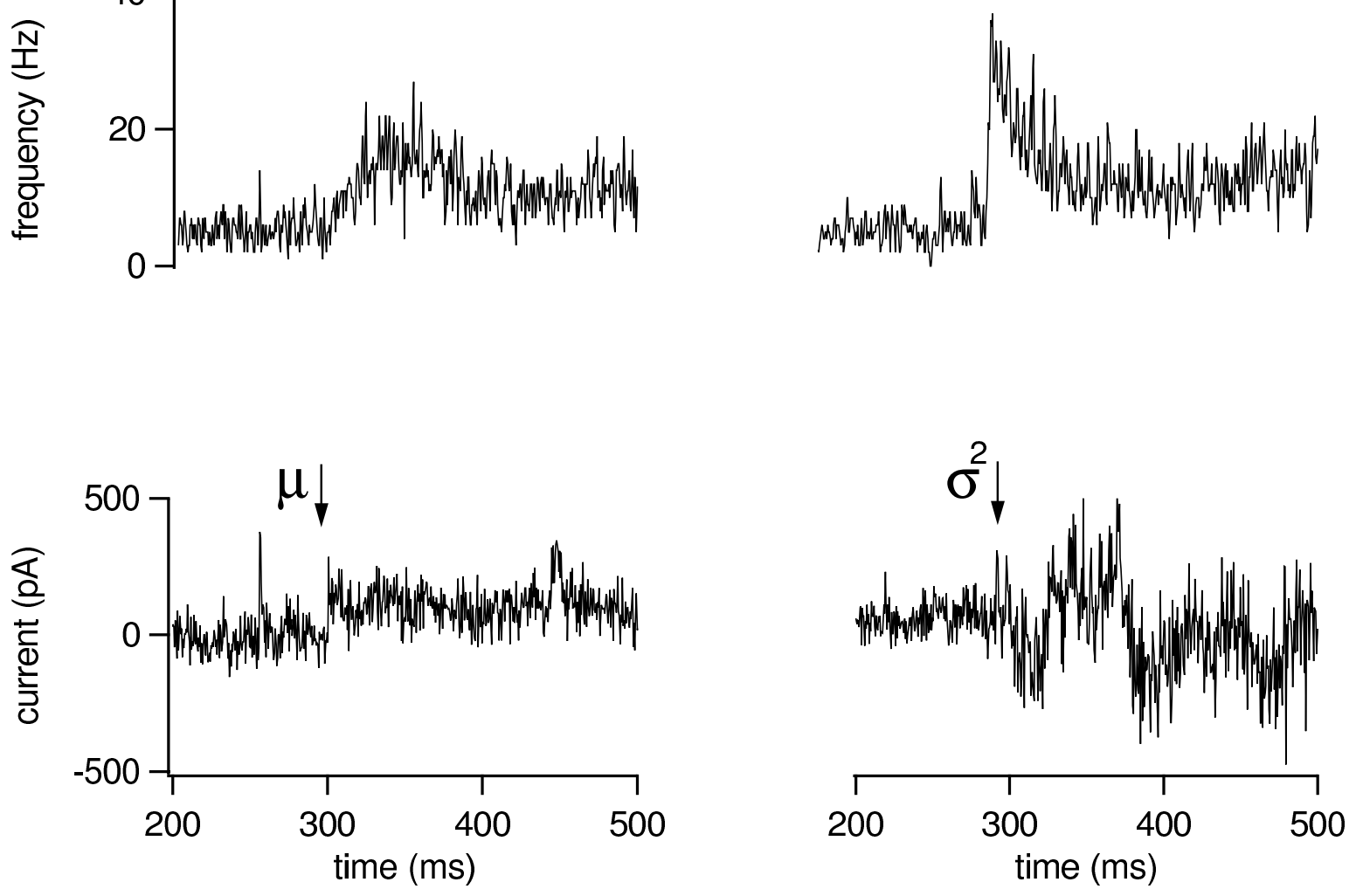

Figure 3.1: Response of a neocortical neuron to currents obtained from voltage-clamp experiments. (A) bottom: A current segment obtained from voltage-clamp experiment. At the transition time, a constant value was added to the current. Top: Histogram of 4000 responses to different current segments. (B) Same as in A but with a step change in the variance of the current. (Figure adapted from Silberberg et al. (2004)) 
For short stimuli, the amplitude had to increase substantially for the faithful prediction, indicating that there might be a contribution of the fast response originating from a large stimulus amplitude (See Fig. 3.2). Intuitively, it is clear that if an input stimulus has a very large amplitude, the neuronal dynamics is completely entrained by the stimulus and the intrinsic neuronal time constants are not limiting the response speed.

Several theoretical studies, however, predicted that the neuronal population response should, even in the limit of small stimulus amplitudes, be instantaneous. In the following section, I will discuss these theoretical studies.

\subsection{Theoretical predictions of dynamic neuronal responses}

Both experiments described above indicate that coding in the variance of the fluctuating input is more efficient in the sense that much higher frequencies can be supported by the neuronal population compared to a coding scheme which is based on modulating the mean input current. While in the first study only one pair of stimulus amplitudes was used, the second study also investigated the dependence of the coding limit on the amplitude of the stimulation. Despite the ability of cortical neurons to respond faithfully to stimuli which don't last as long as the membrane integration time, the study shows that there is nevertheless a lower limit in the stimulus duration which can be coded faithfully. As shown further, this limit can only be overcome by increasing the stimulus amplitude.

The experiments described above are technically demanding and it is thus difficult and time-consuming to assess the dependence of the response properties on all stimulus parameters. Therefore, and because assessing the time limits of population coding is an interesting theoretical problem in itself, there have been many attempts to model the dynamic response properties of populations of cortical neurons using simplified models which incorporate only few dynamical degrees of freedom and which are thus more simple to analyze. In the following section, I will discuss the general approach which was used in previous studies to calculate the response properties of neuronal populations. I will then briefly discuss the class of integrate-and-fire (IF) models and its most prominent representative, the leaky IF (LIF) model, for which the dynamic response properties were previously assessed. Subsequently, I will summarize the main predictions on the dynamic response properties of neurons with fluctuating input currents and explain why the class of IF models fails to explain the experimental results.

\subsubsection{Ensemble dynamics and the linear response regime}

As derived in Sec. 2.8.1, the synaptic input to a single cortical neuron can be described as a randomly fluctuating conductance with a given statistics. To assess the population coding properties in theoretical studies, it is therefore natural to consider ensemble averaged quantities. This approach is schematically depicted in Fig. 3.3. A large population of independent neurons receives an individual fluctuating input which shares the same statistical properties. The assumption behind this approach is that neurons in the cortex are connected only very sparsely, such that the synaptic input each neuron receives is spatially uncorrelated across the population. In addition, a stimulus is fed into the population in parallel. This stimulus can be, as in the experiments described above, a modulation of the mean input current, or a time-dependent modulation of the variance of the fluctuating input. For neurons in the cortex, the external stimulus could be e.g. a moving grating if the neuronal population 
A
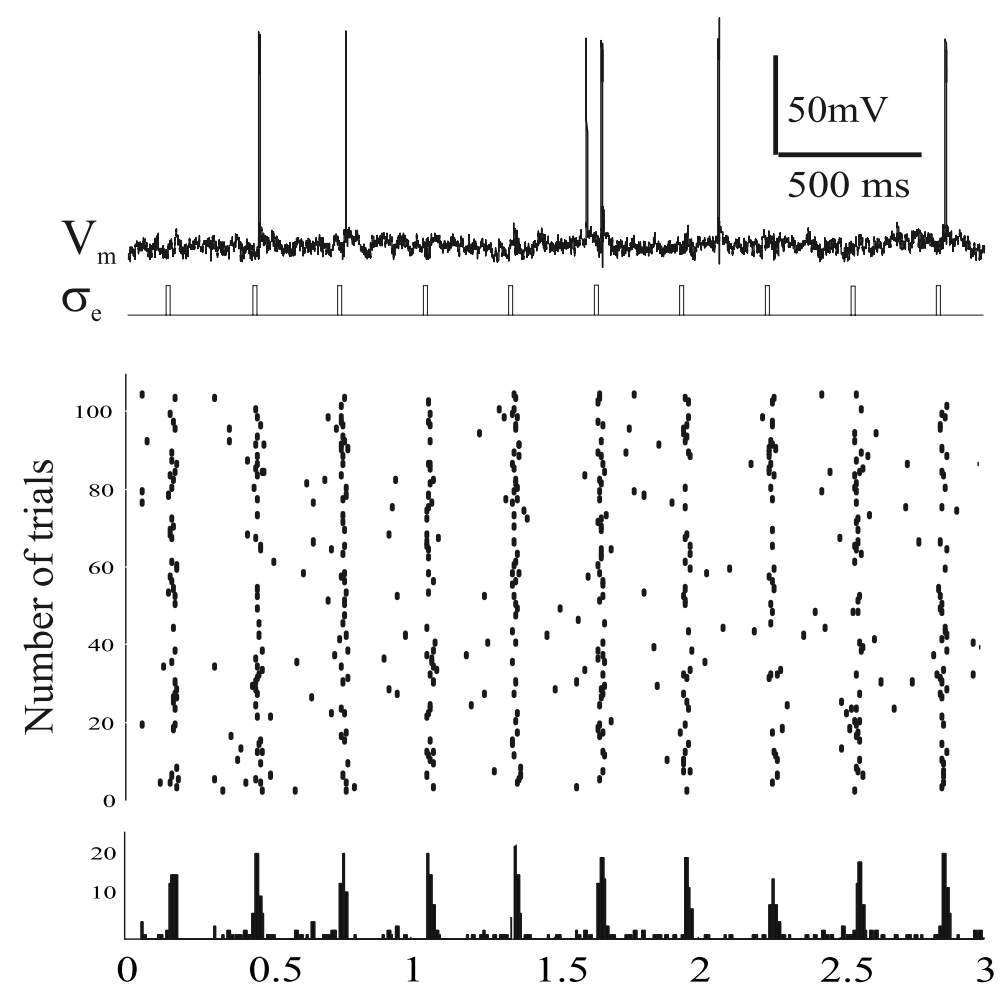

B

Time (s)
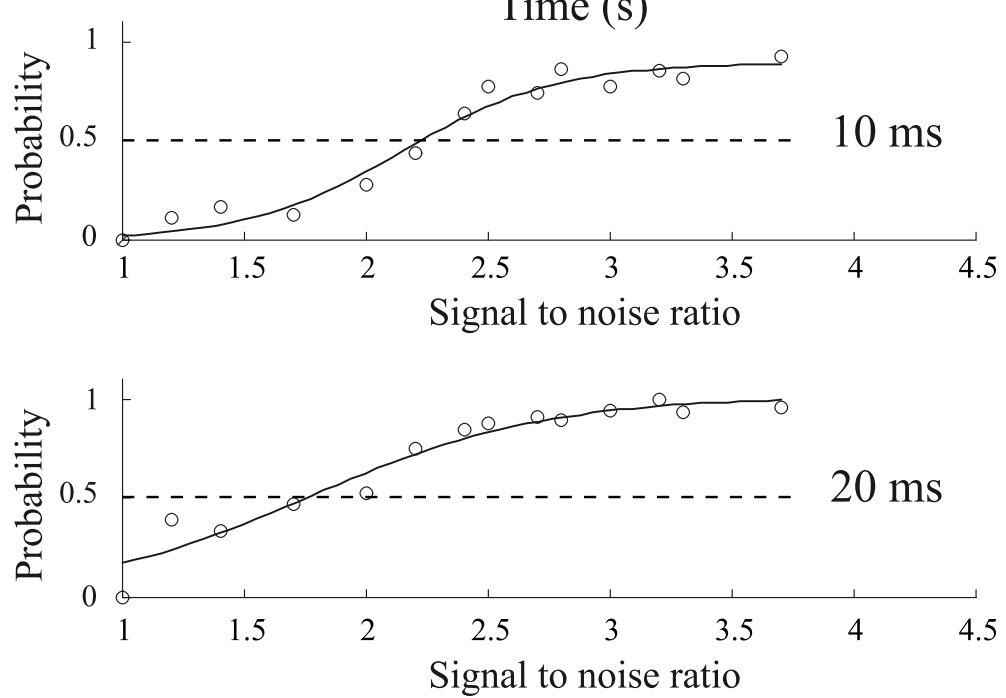

Figure 3.2: Detection in the transients of synaptic inputs. (A) Background synaptic noise was injected into a pyramidal cell. The standard deviation of the excitatory and inhibitory conductances was doubled for a duration of $30 \mathrm{~ms}$ at a rate of $3 \mathrm{~Hz}$, mimicking the arrival of synaptic inputs. The stimulation can be well detected in the population response, as many neurons emit a spike time locked to the signal onset. Top: Sample trace. Middle: Spike rastergram with about 100 trials shown. Bottom: Spike histogram $(10 \mathrm{~ms})$ bins of the rastergram above, showing clear peaks at the time of stimulus onset. (B) Signal detection capability (probability that an AP indicated the presence of a transient input) for varying signal to noise ratios and two different transient lengths. For shorter pulses, the amplitude of the pulse had to be much larger to be able to detect a pulse. (Adapted from Fellous et al. (2003)) 

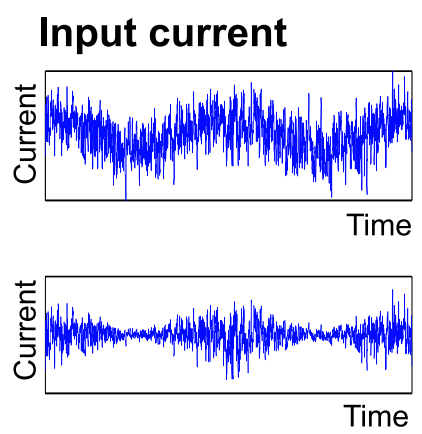
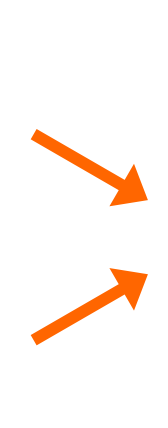

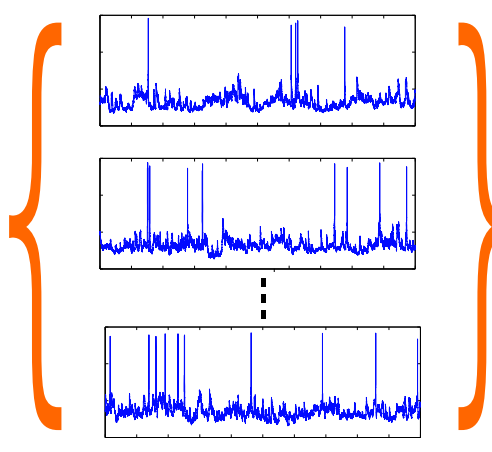

Output rate

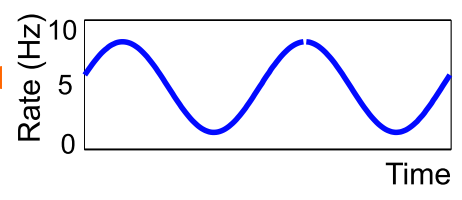

Figure 3.3: Sketch of the population response paradigm. An ensemble of neurons receives a modulated noisy input current or a current, where the noise amplitude is modulated. The noise realization which each neuron receives is different, leading to different MP traces and AP sequences. The output quantity is the population averaged firing rate in the interval $[t, t+d t), v(t)$. As the modulation amplitude is assumed to be sufficiently small, the output rate will be modulated with the same frequency. Thus the relative amplitude and the phase shift with regard to the input signal is sufficient to completely characterize the transmission properties of the neuronal ensemble.

was a hypercolumn from the visual cortex. The output of the population is then given by the ensemble averaged rate $v(t)$ in the interval $[t, t+d t)$.

This approach to assess the time-dependent response properties of a population of neurons is very general. For arbitrary input stimuli, it, however, turns out that it is in most cases impossible to calculate the response properties analytically. Therefore, all theoretical studies assessed the response properties in the linear response regime. Here, it is assumed that the modulation of the time-dependent firing rate is small compared to the stationary firing rate resulting from the fluctuating synaptic input. In this case, it can be assumed that the modulation of the output rate will depend linearly on the amplitude of the stimulus. This dependence can be described by a response function $r(t)$. It directly relates the modulations of the population rate to the time-dependent stimulus via a convolution with the stimulus time-course:

$$
\delta v(t)=r(t) * S(t)=\int_{-\infty}^{t} r\left(t-t^{\prime}\right) S\left(t^{\prime}\right) d t^{\prime},
$$

where $\delta v(t)$ denotes the modulation of the population rate and $S(t)$ the time course of the stimulus. In Fourier representation, the convolution becomes a simple product:

$$
\delta \tilde{v}(\omega)=\sqrt{2 \pi} \tilde{r}(\omega) \tilde{S}(\omega)
$$

The function $\tilde{r}(\omega)$ is in general complex. Its modulus $|\tilde{r}(\omega)|$ describes the damping of the component with frequency $\omega$, whereas its argument $\arg \tilde{v}(\omega)$ describes the relative phase shift of the response. Previous theoretical studies concentrated on determining this response function (or equivalently its Fourier transform) for different types of synaptic input and different types of external stimuli. Although this might appear as a simple problem, the authors of most studies achieved this only for very special cases, e.g. when assuming fast decaying synaptic currents. For more realistic cases, they even restricted themselves to evaluate the response function only for limiting cases. A very popular limiting case turned out to be the high-frequency limit $\omega \rightarrow \infty$. From the decay of the response function in this limit, various studies made predictions about the limiting response speed of neuronal ensembles. Before summarizing the main results, I will discuss the model class for which all of these results were 
obtained.

\subsubsection{Integrate-and-fire (IF) model}

Already in 1907, Lapicque developed a neuron model, which is still very popular and widely used today (Lapicque, 1907; Abbott, 1999). The derivation of this model was a remarkable achievement given that at the time, neither intracellular recordings of single neurons were possible, nor the biophysical mechanisms which lead to the generation of APs had been discovered.

Lapicque modeled a neuron as an electric circuit consisting of a capacitor and a resistor connected in parallel, which represent the capacitance and the leakage resistance of a neuronal membrane. In this model, a current balance equation describes the dynamics of the MP with an externally applied current $I_{\text {ext }}$ :

$$
C \dot{V}(t)+g_{L}\left(V(t)-V_{0}\right)+I_{\text {ext }}(t)=0,
$$

where the resting potential is denoted by $V_{0}$. This equation is effectively an integrator equation for the external current. As this model equation can not generate APs, Lapique introduced a phenomenological rule: Each time the MP $V(t)$ reaches a threshold potential $V_{\text {thres }}$, a spike is said to be "fired" and the MP is set to some reset value $V_{r}$.

Today, the integrate-and-fire (IF) model is used in a wide variety of studies, ranging from the spiketiming of single neurons up to simulations of large networks. In the latter framework it is particularly popular, due to its few parameters and dynamical simplicity (Diesmann et al., 1999). Over the years, Lapicques' original model was extended into a large class of different models. They all assume a particular form of subthreshold integration and a threshold for the initiation of an AP. In some models, even the assumption of a fixed threshold potential was relaxed and a dynamic threshold voltage was assumed. In chapter 5 of this thesis, I will also introduce a model of this type.

The dynamic response properties of ensembles of neurons were systematically studied exclusively using IF models. This topic was first pioneered by Knight (Knight, 1972; Knight et al., 2000; Knight, 2000), who considered a simple noise-free integrator model in which the firing threshold is either fixed or drawn randomly, every time an AP occurs. His results were later extended to models, where also the reset voltage was drawn randomly, and to models in which the input changed either very slowly or to spike response models, where the input is assumed to change very rapidly (Gerstner, 2000). Only recently was the impact of current noise on the dynamic response of the leaky IF model investigated (Brunel \& Hakim, 1999; Brunel, 2000; Brunel et al., 2001; Lindner \& Schimansky-Geier, 2001). In the next section I will briefly summarize the main results of these studies, which are important to understand the aim of the following analysis.

\subsubsection{Summary of the predictions implied by the leaky IF model}

The linear response properties of the LIF model with a fluctuating input current were derived in three regimes which are characterized by a different statistics of the fluctuating synaptic background and different stimulation paradigms. The response in the fourth regime was not derived before, but directly follows from the analysis of the response in the second regime.

First regime: In the first studies (Brunel \& Hakim, 1999; Brunel, 2000), a Gaussian white background input (i.e. synaptic events with a time constant which is zero) and a stimulation of the 
mean input current was assumed. The authors of these studies showed that in this regime the linear response function $\tilde{r}(\omega)$ typically exhibits a cut-off given by the population averaged firing rate. They also assessed the response in the high frequency limit, where they found that the amplitude of the response function decays proportionally to $1 / \sqrt{\omega}$. The phase shift relative to the stimulation was determined as $\pi / 4$.

Second regime: Because of the unrealistic assumption of the first studies which assumed synaptic inputs with an infinitely fast decay, the authors of a following study assessed the response behavior to a stimulation in the mean input current in the presence of synaptic inputs with a non-zero decay time constant (Brunel et al., 2001). The input currents were modeled as an Ornstein-Uhlenbeck process with a single time constant $\tau$. The authors of this study were not able to calculate the full transmission function analytically, but only the high-frequency limit. They found that for a physiologically realistic time constant of the synaptic input, the response function $\tilde{r}(\omega)$ can even increase for intermediate frequencies and stays constant for larger frequencies, even in the limit $\omega \rightarrow \infty$. The phase shift of the response was shown to go to zero, resulting in a perfect input current to spike rate converter.

Third regime: In a third independent study, the response properties for a stimulus which modulates the variance of the background noise was considered (Lindner \& Schimansky-Geier, 2001). For the sake of analytical tractability, the authors considered, as in the first regime, temporally uncorrelated synaptic currents. In a tedious calculation, they determined the complete response function analytically and showed, similarly to the second regime, that in the high frequency limit, the response function goes to a finite value and the relative phase goes to zero.

Fourth regime: The result obtained in the previous regime does also hold for the case of realistic synaptic inputs. However, the mathematical structure for the derivation of the high-frequency limit is almost identical to the second regime. Also in this regime it is easily shown that the response amplitude is finite in the high frequency limit and the relative phase of the response goes to zero.

The results in these four regimes were derived for the LIF model. In particular, the results obtained in the second, third and fourth regime apply, however, for the whole class of IF models, as shown by the authors of the corresponding studies. The only condition necessary for obtaining the response behavior is a fixed threshold for the initiation of APs. This means that the results obtained for the four regimes are typical for the large class of IF models.

\subsubsection{Failure of IF models to describe the experimental results}

Let us recall the outcome of the experiments in Sec. 3.2.1 and 3.2.2. In the first experiment, the response for the coding in the variance of the noise input seemed to be as fast as resolvable by the resolution of the recording, i.e. on the order of $1 \mathrm{~ms}$. Indeed, the authors of this study explained their experimental results with the response behavior of the LIF model in the third regime. However, this explanation is flawed for two reasons:

1. The background synaptic input, which was injected, was obtained from the recordings of real synaptic currents. This input was therefore temporally correlated. The expected response behavior should therefore be according to the second regime. This means that if the LIF model 
was a suitable model for the dynamics of the cortical neurons assessed, the response to a step in the mean input current or in the amplitude of the fluctuations should have had the same effect: An instantaneous response of the population averaged time-dependent firing rate.

2. The response properties of the LIF model were assessed in the linear response regime. From the second experiment we learned, however, that the response speed partially depends on the amplitude of the stimulation. This suggests that the response of real neurons for very small stimuli, for which the response of the LIF model was derived, may be much slower.

In the following section, a generic phase oscillator model, the generalized $\theta$-neuron, is introduced. I will show that this model can, in contrast to the IF models, reproduce the experimental results. Moreover, it predicts a direct connection between the dynamic AP onset dynamics and the response speed of a neuronal population.

\subsection{The generalized $\theta$-neuron: A generic phase oscillator model}

Phase oscillator models have a long history in physics and biology (Coddington \& Levinson, 1955; Winfree, 1967; Guckenheimer, 1975; Winfree, 2001). Recently, they were also introduced in theoretical neuroscience (Ermentrout \& Rinzel, 1984). In contrast to IF models, which are phenomenological models of cortical neurons, they can be derived from the limit cycle dynamics of conductance-based models, thereby reducing the complex dynamics which usually incorporates many degrees of freedom to a single phase variable.

In the following, I will study the stationary and dynamic response properties in a particular phase oscillator model, a generalization of the $\theta$-neuron which models excitable neuronal membranes exhibiting Type-I excitability.

\subsubsection{From normal forms to phase oscillators}

When brought to repetitive firing, conductance-based neuron models which exhibit Type-I excitability typically undergo a saddle-node bifurcation of codimension one (Rinzel \& Ermentrout, 1998). A center-manifold reduction at the bifurcation point leads to a normal form of the dynamics at the bifurcation (Strogatz, 2001):

$$
C \dot{\bar{V}}=\frac{g}{V_{0}}\left(\bar{V}-V^{*}\right)^{2}+\left(\bar{I}(t)-I_{e}\right),
$$

which is a dynamical equation for the MP $\bar{V}$ of the neuron. Here, the input current relative to the rheobase $I_{e}$ of the neuron is denoted by $\bar{I}(t)$. The constants $g, V_{0}$ and $V^{*}$ can, in principle, be deduced from a given multidimensional conductance-based neuron model (an explicit example is given e.g. in (Hansel \& Mato, 2003)). For the following analysis, it is convenient to introduce dimensionless quantities $V(t)$ and $I(t)$ :

$$
\begin{aligned}
V(t) & =\left(\bar{V}(t)-V^{*}\right) / V_{0} \\
I(t) & =\left(\bar{I}(t)-I_{c}\right) /\left(g V_{0}\right)
\end{aligned}
$$

and the effective time constant:

$$
\tau=C / g
$$




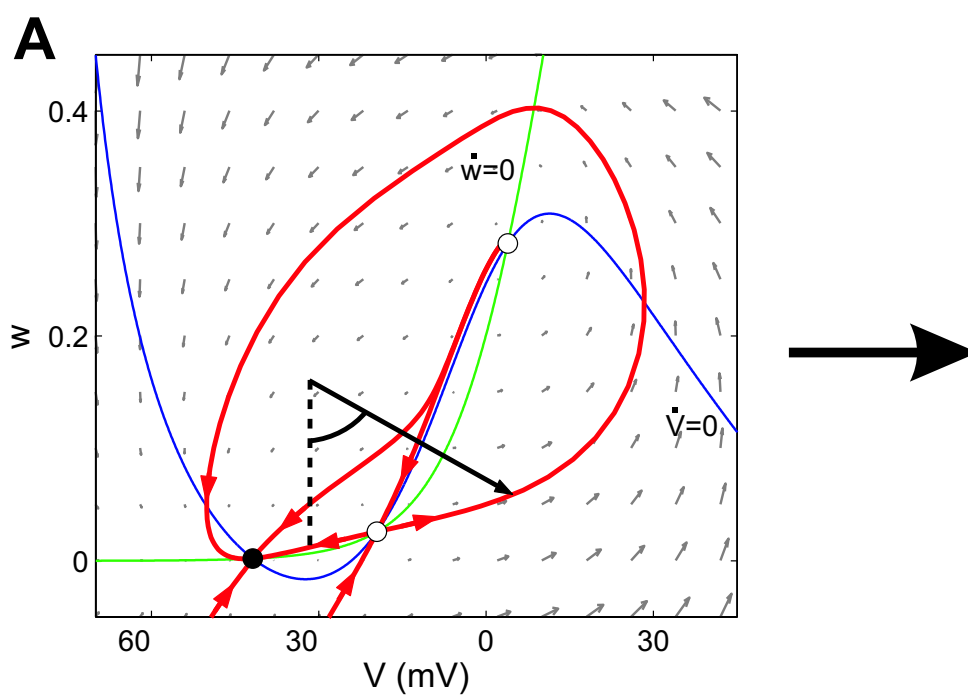

B

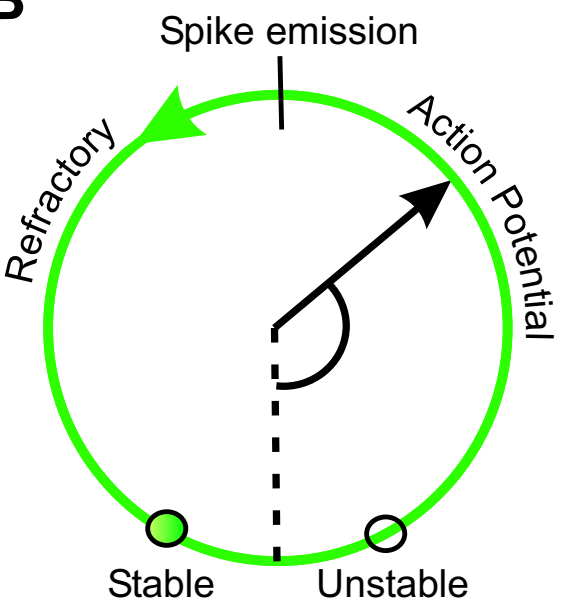

Figure 3.4: (A) Phase plane of a type-I single compartment conductance-based model (Morris-Lecar model (Morris \& Lecar, 1981)) in the excitable regime (filled dot: stable fixed point, open dots: unstable fixed points). The blue and green lines are the $\dot{V}=0$ and the $\dot{w}=0$ nullclines, respectively. The red lines are the stable and unstable manifolds of the saddle and the node. (B) The excitable dynamics can be reduced to a phase oscillator with one degree of freedom parameterized by the angle $\theta$. To initiate an AP, the phase has to surpass the unstable fixed point. At some angle $\theta_{s}$, a spike is said to be emitted and the phase relaxes back to the stable fixed point.

Using these substitutions, the rescaled dynamics is given by:

$$
\tau \dot{V}=V^{2}+I(t)
$$

For $I(t)>0$ the dynamics has a finite "blow-up" time, meaning that it needs a finite time to get from $-\infty$ to $+\infty$. To turn this model into an oscillator, both ends of the real axis are identified and at some value $V_{s}$ a spike is said to be emitted. For constant input currents $I(t)=I_{0}$, the spike rate is given by:

$$
v=\tau^{-1}\left(\int_{-\infty}^{\infty} \frac{d V}{V^{2}+I_{0}}\right)^{-1}=(\tau \pi)^{-1} \sqrt{I_{0}}
$$

This square-root dependence of the firing rate on the input current is a salient feature of Type-I excitable membranes. As shown by Tateno et al. (2004), this dependence also reflects well the dynamics of cortical pyramidal neurons in vitro, when constant currents are injected.

The normal form Eq. (3.8) is equivalent to a phase oscillator, the $\theta$-neuron (Ermentrout \& Rinzel, 1984; Gutkin \& Ermentrout, 1998). Its equation of motion,

$$
\tau \dot{\theta}=(1-\cos \theta)+I(t)(1+\cos \theta),
$$

is found by substituting $V=\tan (\theta / 2)$ with the angle variable $\theta$ in the interval $(-\pi, \pi]$. In the model, a spike is emitted each time $\theta$ reaches the value $\theta_{s}$. By choosing $\theta_{s}=\pi$, the original $\theta$-neuron is obtained. In Fig. 3.4, the reduction of a conductance-based neuron model to a phase oscillator model is illustrated schematically.

What is the main difference between this oscillator model and the class of IF models? As discussed 
above, it is assumed in IF models that APs are generated every time the MP reaches a fixed threshold, which leads to the observed artificial response behavior. In this phase oscillator model on the other hand, the AP dynamics is explicitly included in the model. To generate an AP, an unstable fixed-point has to be crossed. This dynamical feature is much more typical than a threshold dynamics.

\subsubsection{The generalized $\theta$-neuron}

Mathematically, the $\theta$-neuron describes the dynamics of the AP generation close to the bifurcation. The model lacks, however, a crucial feature found in real neurons (cf. chapter 4). The relaxation dynamics close to the stable fixed point and the dynamics at the AP onset is assumed to have the same time constant. Real neurons, however, show a strong asymmetry in the relaxation dynamics and AP onset dynamics, the dynamics at AP onset is typically much faster than the relaxation dynamics. To account for this fast onset dynamics, I generalized the $\theta$-neuron model in the following way:

$$
\tau \dot{V}=V^{2}+I(t)+\alpha(1+\tanh (\beta V)),
$$

where the two additional parameters $\alpha$ and $\beta$ are introduced. The sigmoidal term phenomenologically models the part of the sodium channel activation curve, which is not included in the $V^{2}$-term of the normal form. The parameter $\alpha$ controls the sodium peak conductance and the parameter $\beta$ the width of this activation curve. Both parameters control the rapidness of the AP onset. As for the normal form, an equivalent phase oscillator equation can be found by substituting $V=\tan (\theta / 2)$ :

$$
\tau \dot{\theta}=(1-\cos \theta)+(1+\cos \theta)\{I(t)+\alpha(1+\tanh (\beta \tan (\theta / 2))\}
$$

\subsection{Fokker-Planck equation for the dynamics of neuronal populations}

As described in Sec. 2.8.1, neurons in vivo are subject to an ongoing synaptic bombardment, resulting in a fluctuating MP. To model this situation, we assume a temporally fluctuating input current as derived in Sec. 2.8.2:

$$
I(t)=I_{0}+\sigma z(t),
$$

composed of a mean current $I_{0}$ and a stationary fluctuating part $\sigma z(t)$, where $z(t)$ is a OrnsteinUhlenbeck process with the correlation time $\tau_{c}$.

Eq. 3.10 and Eq. 3.12 describe a realization of the dynamics of a single neuron. To compare the model dynamics with the dynamic firing rate response measured in the experiments, a population of neurons is considered in the following. The state of this population is naturally described by the timedependent probability density function (pdf) $P(\theta, z, t)$, which is the probability of finding a single neuron at time $t$ in the interval $[\theta, \theta+d \theta) \times[z, z+d z)$ (Risken, 1996). The dynamics of the pdf is determined by the Fokker-Planck operator $\hat{L}$ :

$$
\partial_{t} P(\theta, z, t)=\hat{L} P(\theta, z, t),
$$


with,

$$
\begin{aligned}
\hat{L}= & -\tau^{-1} \partial_{\theta}\left\{(1-\cos \theta)+\left(I_{0}+\sigma z+\alpha(1+\tanh (\beta \tan (\theta / 2)))\right)\right. \\
& \cdot(1+\cos \theta)\}+\tau_{c}^{-1} \partial_{z}\left(z+\frac{\tau}{2 \tau_{c}} \partial_{z}\right)
\end{aligned}
$$

The boundary conditions for $P(\theta, z, t)$ are periodic in the $\theta$ - and natural in the $z$-direction:

$$
\begin{aligned}
P(-\pi, z, t) & =P(\pi, z, t) \\
\partial_{\theta} P(-\pi, z, t) & =\partial_{\theta} P(\pi, z, t) \\
\lim _{z \rightarrow \pm \infty} P(\theta, z, t) & =0 \\
\lim _{z \rightarrow \pm \infty} \partial_{\theta} P(\theta, z, t) & =0
\end{aligned}
$$

\subsubsection{The limit of fast-decaying synaptic currents}

It turns out that it is practically impossible to give closed expression even for the stationary response properties in the case that the input current is correlated. However, in the limit $\tau_{c} \rightarrow 0$ the input current becomes Gaussian white noise and the Fokker-Planck operator, which describes the dynamics of the pdf $P(V, t)$ is given by:

$$
\tau \partial_{t} P(V, t)=-\partial_{V}\left(V^{2}+I_{0}+\alpha(1+\tanh (\beta V))\right) P(V, t)+\frac{\sigma^{2}}{2} \partial_{V}^{2} P(V, t)
$$

The equivalent Fokker-Planck equation for the pdf $P(\theta, t)$ is, however, not uniquely defined. The problem that arises is the multiplicative dependence of the noise source on the angle variable $\theta$ in the corresponding Langevin Eq.:

$$
\tau \dot{\theta}=(1-\cos \theta)+(1+\cos \theta)\left(I_{0}+\tau^{-1 / 2} \eta(t)\right)
$$

Langevin Eqs. of this type have to be "interpreted". A very thorough and exhaustive discussion on this issue can be found e.g. in (van Kampen, 1981). It can be shown that in the limit of correlated input noise with a vanishing correlation time, the so-called Stratonovich interpretation has to be chosen. The resulting Fokker-Planck equation is then given by:

$$
\begin{aligned}
\tau \partial_{t} P(\theta, t)= & -\partial_{\theta}\left((1+\cos \theta)\left(I_{0}+\alpha(1+\tanh (\beta \tan (\theta / 2)))\right)\right) \\
& +\frac{\sigma^{2}}{2} \partial_{\theta}(1+\cos \theta) \partial_{\theta}(1+\cos \theta) P(\theta, t)
\end{aligned}
$$

This Eq. can be reformulated as:

$$
\begin{aligned}
\tau \partial_{t} P(\theta, t)= & -\partial_{\theta}\left((1-\cos \theta)\left(I_{0}+\alpha(1+\tanh (\beta \tan (\theta / 2)))-\frac{\sigma^{2}}{2} \sin \theta\right)\right) \\
& +\frac{\sigma^{2}}{2}(1+\cos \theta) \partial_{\theta}^{2}(1+\cos \theta) P(\theta, t)
\end{aligned}
$$

The additional contribution $-\sigma^{2} / 2 \sin \theta(1+\cos \theta)$ in the drift term is often called "spurious drift", because it just arises from the multiplicative nature of the noise. 


\subsubsection{Time-dependent firing rate}

Intuitively, the time-dependent firing rate can be deduced very easily from the flux, which is given by the first term of the Fokker-Planck Eq. (3.22). It is given by the probability current across the line $\theta=\theta_{s}$ which has positive velocity $\dot{\theta}>0$. For $\theta_{s}=\pi$, it is possible to give an explicit expression for the firing rate. There, the dynamics is insensitive of the external input current $I(t)$ and all trajectories have the same velocity $2 \tau^{-1}$. Therefore, at $\theta_{s}=\pi$ the firing rate is exactly equal to the probability current through the entire line $\theta=\pi$ :

$$
v(t)=2 \int_{-\infty}^{\infty} d z P(\pi, z, t)
$$

Although the choice $\theta_{s}=\pi$ is quite convenient for analytical considerations, this spike-phase is rather arbitrary. In the normal form Eq. (3.8), the point $\theta_{s}=\pi$ corresponds to the point $V=\infty$, where the model reflects least the dynamics at the bifurcation. To assess if this particular choice has any influence on the dynamic response properties of the model, I will also calculate the firing rate at $\theta_{s}=\pi-\delta$. The probability current through this line is given by:

$$
\begin{aligned}
J_{\theta}= & \tau^{-1} \int_{-\infty}^{\infty} P\left(\theta_{s}, z, t\right)\left(\left(1-\cos \theta_{s}\right)+(1+\cos \theta) .\right. \\
& \left(I_{0}+\sigma z+\alpha\left(1+\tanh \left(\beta \tan \left(\theta_{s} / 2\right)\right)\right)\right) d z
\end{aligned}
$$

The firing rate is, however, not exactly given by the flux $J_{\theta}$, as we did not take into account the condition that only trajectories should be counted which have a positive velocity. Naturally, there is a contribution from trajectories which are driven back below the threshold due to the external fluctuations. For a correlated input current, however, the introduced error is exponentially small. This can be seen in Eq. (3.12). For small values of $\delta$, the probability distribution $P(\theta, \dot{\theta})$ around $\pi-\delta$ is a Gaussian with a mean value $2-\delta^{2}$ and a width $\propto \delta^{2}$. The negative part of this Gaussian is proportional to:

$$
\left(2 \pi \delta^{4} \sigma^{2}\right)^{-1 / 2} \int_{-\infty}^{0} \exp \left(-\frac{\left(x-\left(2-\delta^{2}\right)\right)^{2}}{2 \delta^{4} \sigma^{2}}\right) d x
$$

For all practical purposes $(\delta<0.5$ and $\sigma<1)$, this integral is smaller than $10^{-10}$. It will turn out, however, that the definition $\theta_{s}=\pi$ does qualitatively change the high-frequency limit of the dynamic response behavior.

In the limit $\tau_{c} \rightarrow 0$, i.e. fast decaying synapses, the probability current is given by:

$$
J_{\theta}^{\left(\tau_{c} \rightarrow 0\right)}=\tau^{-1}\left\{(1-\cos \theta)\left(I_{0}+\alpha(1+\tanh (\beta \tan (\theta / 2)))\right)-\frac{\sigma^{2}}{2}(1+\cos \theta) \partial_{\theta}(1+\cos \theta)\right\} P(\theta, t)
$$

For a spike emission at $\theta_{s}=\pi$, the firing rate is, as before, identical to the current and given by:

$$
v^{\left(\tau_{c} \rightarrow 0\right)}=2 \tau^{-1} P(\pi, t)
$$

As in the case $\tau_{c}>0$, for $\theta_{s} \neq \pi$, the rate is not any longer given by the current. It can be shown that, in this case, the introduced error is however proportional to $\left(\theta_{s}-\pi\right)^{2}$. 


\subsubsection{Parameter choice}

So far, I have not commented on a sensible choice of the model parameters. Because it is not possible to give explicit analytical results for the stationary and dynamic response quantities, it is even more important to define a biologically plausible parameter regime in which these quantities are approximated or evaluated numerically. The parameters which have to be fixed are the time constant $\tau$, the mean input current $I_{0}$, the strength of the fluctuating input $\sigma$ and the synaptic input correlation time $\tau_{c}$.

In the following I will discuss the generalized $\theta$-neuron in three different regimes, namely in the subthreshold, critical and suprathreshold regime. In the subthreshold regime, an estimate of the correlation time of the MP is given by approximating the dynamics for $I_{0}<0$ near the stable fixed point by an Ornstein-Uhlenbeck process. Straightforward linearization around the stable fixed point at $-\sqrt{\left|I_{0}\right|}$ then yields:

$$
\tau_{\text {relax }} \approx \tau\left(2 \sqrt{\left|I_{0}\right|}\right)^{-1}
$$

In the subthreshold noise-driven regime, I choose $I_{0}=-0.1$. The time constant $\tau$ is then adapted via Eq. (3.29), to achieve a relaxation time of approximately $5 \mathrm{~ms}$, which leads to values for $\tau$ of approximately $3 \mathrm{~ms}$.

In the critical regime, i.e. $I_{0}=0$, a realistic relaxation time is achieved by choosing a smaller value of $\tau \approx 0.25$, which I also used in the suprathreshold regime.

The parameters $\alpha$ and $\beta$ parameterize the speed of the AP onset. For the following numerical treatment, I keep $\beta$, which mediates the width of the activation curve and is an intrinsic physiological parameter, fixed to a value of 20 . The parameter $\alpha$, which represents the sodium peak conductance, is changed in the range from 0 to 1 . At this point, I will not fit the parameters $\alpha$ and $\beta$ to the dynamics of a particular neuron model. In chapter 4 , the onset dynamics of cortical APs is discussed in detail.

In Fig. 3.5, three sample realizations of Eqs. $(3.12,3.13)$ are shown for different values of the parameter $\alpha$. If the input current is positive for a sufficient amount of time, APs are initiated. With increasing values of $\alpha$, the sharpness at the onset increases, while the subthreshold fluctuations are not affected.

\subsection{Stationary response properties}

I will first discuss the stationary response properties of the generalized $\theta$-neuron. For the case of realistic synaptic input, i.e. $\tau_{c}>0$, it is not possible to give exact analytical expressions for the firing rate. For the $\theta$-neuron, only approximate solutions for $\tau_{c} \ll \tau$ or $\tau_{c} \gg \tau$ were derived (Brunel \& Latham, 2003). This problem is closely linked to the Kramers' escape rate problem with correlated input noise, for which also only approximate solutions were found so far. It is, however, possible to compute the stationary properties in a very elegant semi-analytical approach using a sparse matrix representation of the Fokker-Planck operator.

Nevertheless, it is important e.g. for analytical studies on neural network dynamics (see e.g. (Brunel \& Hakim, 1999)) to have explicit analytical expressions at hand for the stationary firing rate. In this section I will therefore derive expressions for the rate in the limit of fast-decaying synaptic currents. 


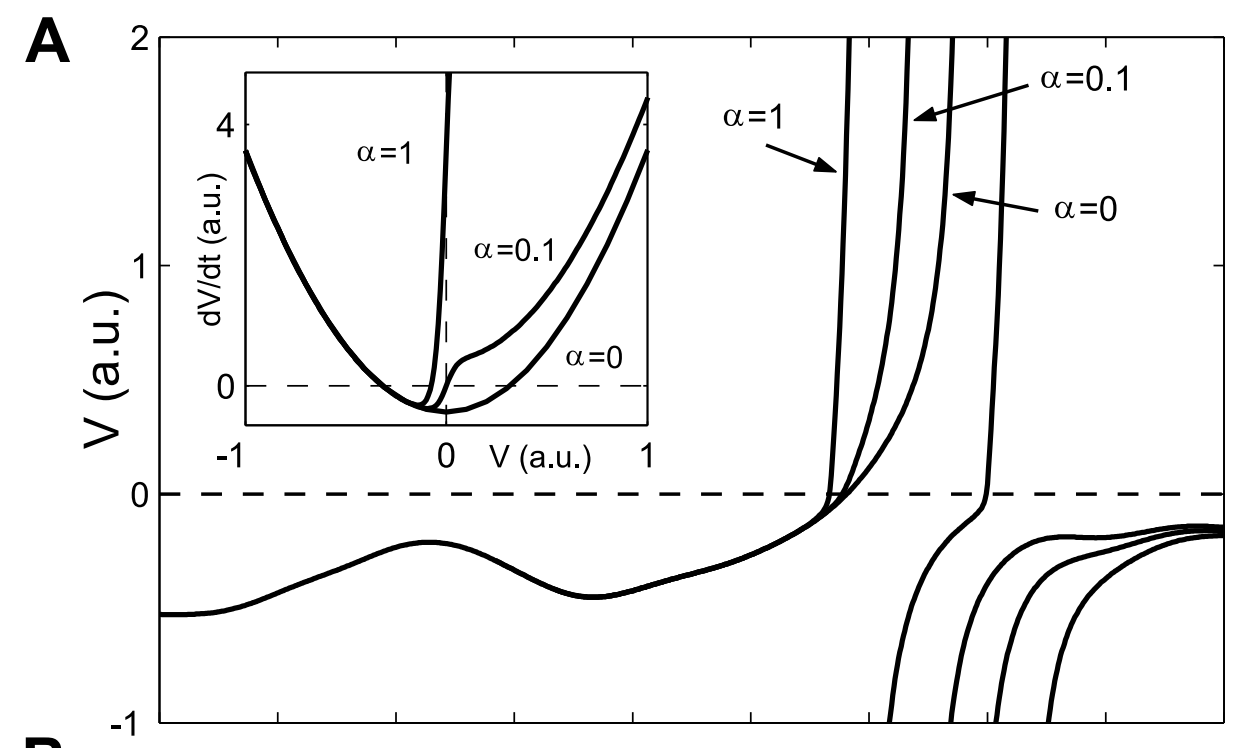

B

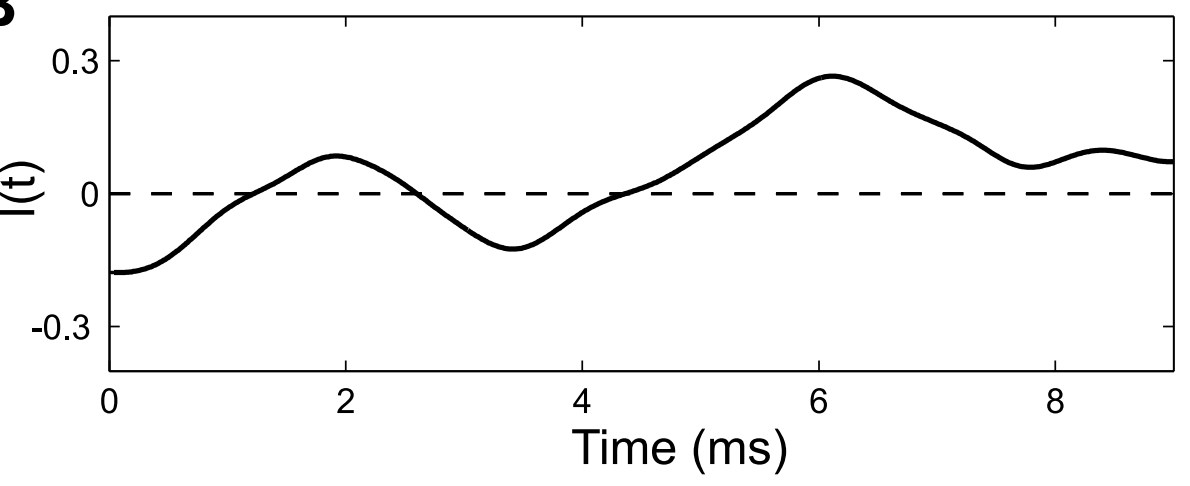

Figure 3.5: Dynamics of the generalized $\theta$-neuron model for different values of the AP onset parameter $\alpha$. An increasing value of the parameter $\alpha$ leads to a sharper AP onset. (A) Sample MP trajectories for $\alpha=0, \alpha=0.1$ and $\alpha=1$. The inset shows the deterministic part of Eq. (3.12). (B) Fluctuating input current $I(t)$. The parameters are: $\tau_{c}=1.5 \mathrm{~ms}, \sigma=0.3, I_{0}=-0.1$ and $\beta=20$. Immediately before the AP onsets, the subthreshold fluctuations of the MP are virtually identical. For $\alpha=1$, the model generates two APs. 


\subsubsection{Firing rate in the limit of fast-decaying synaptic currents}

In the stationary case, the pdf $P(V, t)$ becomes time-independent. It can then be shown that the probability flux given by Eq. (3.27) is independent of $V$. By replacing $P(V, t)$ by the stationary pdf $P_{0}(V)$ and subsequent integration, the stationary pdf and firing rate for the case $\alpha=0$ is given by:

$$
\begin{gathered}
P_{\mathrm{st}}(V)=\frac{2 v \tau}{\sigma^{2}} \int_{V}^{\infty} d V^{\prime} \exp \left\{\frac{2}{\sigma^{2}}\left(\frac{1}{3}\left(V^{3}-V^{\prime 3}\right)+I_{0}\left(V-V^{\prime}\right)\right)\right\} \\
v^{-1}=\frac{2 \tau \sqrt{2 \pi}}{\sigma} \int_{0}^{\infty} d y \exp \left\{-\frac{2}{\sigma^{2}}\left(\frac{y^{6}}{12}+I_{0} y^{2}\right)\right\}
\end{gathered}
$$

The expression for the rate is deduced with the additional constraint that $\int d V P_{\mathrm{st}}(V)=1$.

For $\alpha>0$, the density and firing rate are given by:

$$
\begin{aligned}
P_{\mathrm{st}}(V)=\frac{2 v \tau}{\sigma^{2}} \int_{V}^{\infty} \exp \left\{\frac{2}{\sigma^{2}}\left(\frac{1}{3}\left(V^{3}-V^{\prime 3}\right)+\left(I_{0}+\alpha\right)\left(V-V^{\prime}\right)+\frac{\alpha}{\beta}\left(\ln \cosh V-\ln \cosh V^{\prime}\right)\right)\right\} d V^{\prime}, \\
v^{-1}=\frac{2 \tau}{\sigma^{2}} \int_{\infty}^{\infty} d V \int_{V}^{\infty} d V^{\prime} \\
\quad \exp \left\{\frac{2}{\sigma^{2}}\left(\frac{1}{3}\left(V^{3}-V^{\prime 3}\right)+\left(I_{0}+\alpha\right)\left(V-V^{\prime}\right)+\frac{\alpha}{\beta}\left(\ln \cosh V-\ln \cosh V^{\prime}\right)\right)\right\} \cdot(3.33)
\end{aligned}
$$

Due to the additional term, which tunes the AP onset dynamics, the stationary rate is given by a two-fold integral, which can not be simplified any further. In Fig. 3.6, the stationary pdf and firing rate are shown for different values of $\alpha$ and increasing noise amplitude and input current. The firing rate exhibits two characteristic features. Firstly, it increases gradually with increasing values of the noise amplitude and a constant input current. In contrast to the deterministic model there is no critical current below which the neuron model does not produce any APs. Secondly, the firing curve preserves the square-root type dependence, which is typical for cortical non-adapting pyramidal neurons. For increasing values of $\alpha$, the stationary pdf exhibits a cut-off of increasing sharpness at $\theta=0$, reflecting a more rapid AP onset. For a fixed noise amplitude $\sigma$ and an external current $I_{0}$, the firing rate increases as a function of $\alpha$ and the onset of oscillatory firing already starts at smaller values of $I_{0}$ and $\sigma$.

As outlined in the introduction, the knowledge of the mean firing rate is a crucial ingredient for the analytical understanding of self-consistent network theories. For these studies, it is however important to have simple explicit expressions for the firing rate. This is unfortunately not possible for most neuron models, including the $\theta$-neuron model. In the remainder of this section, I will therefore derive approximate solutions for the firing rate in three regimes (low rate, intermediate rate, high rate regime) in the limit $\alpha=0$.

\subsubsection{Low-rate approximation}

The rate $v$ obeys the scaling relation $v\left(I_{0}, \sigma\right)=\sigma^{2 / 3} v\left(\sigma^{-4 / 3} I_{0}, 1\right)$. This means that in the following discussion we can, without loss of generality, set $\sigma \equiv 1$. In the limit of low rates, i.e. negative $I_{0}$ the 
A

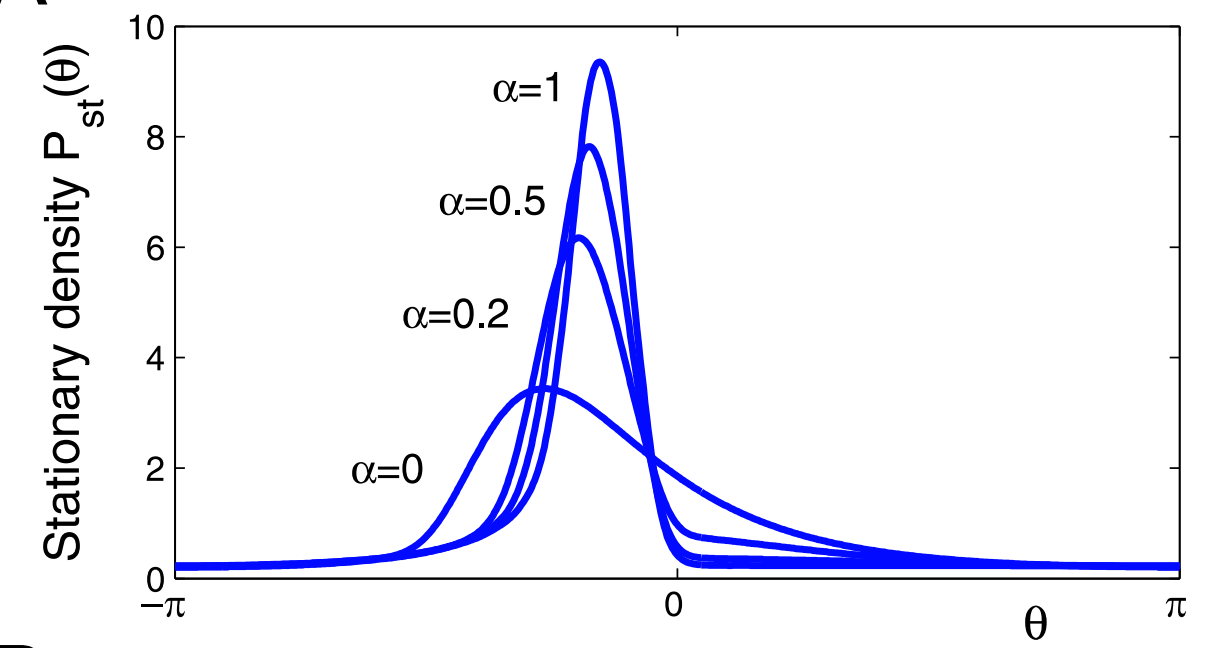

B

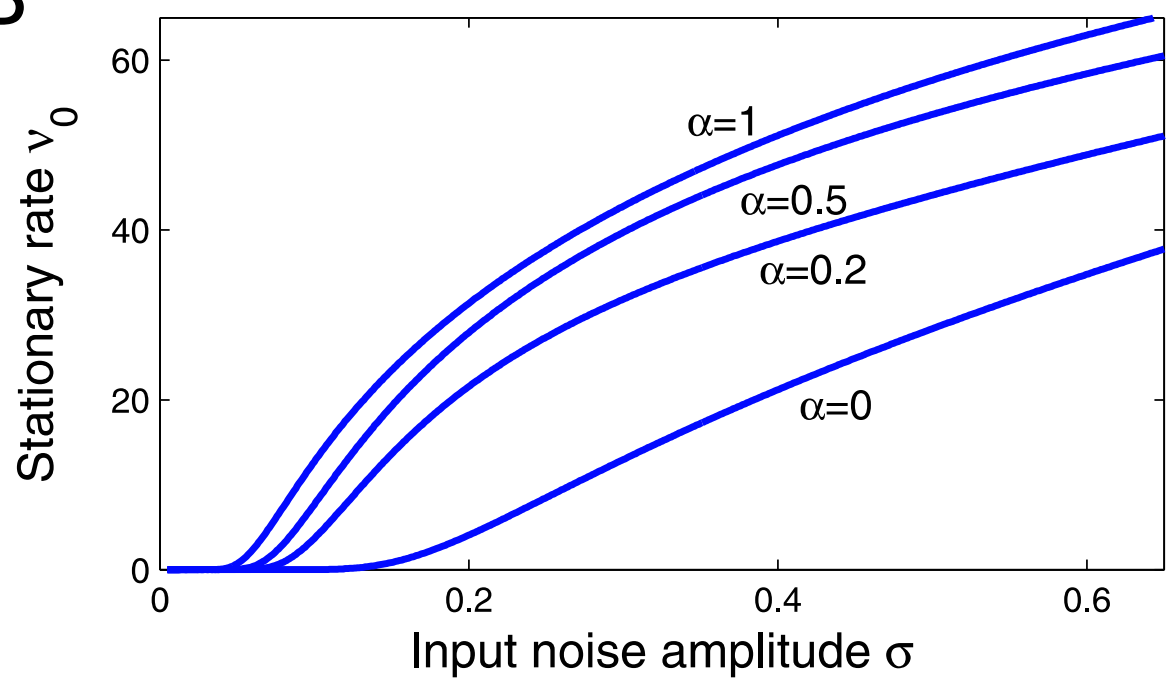

Figure 3.6: (A) Stationary density $P_{\mathrm{st}}(\theta)$ and (B) rate $v$ for increasing values of $\alpha$. In (A), $\sigma$ was adjusted to achieve a constant rate of $30 \mathrm{~Hz}$. The other parameters are $I_{0}=-0.1$ and $\beta=20$. For increasing values of $\alpha$, the pdf exhibits a sharp decay around $\theta=0$. The output rate $v$ increases for a fixed value of $\sigma$ and increasing values of $\alpha$. 
exponent can be expanded in a saddle point approximation:

$$
v^{-1}=2 \tau \sqrt{2 \pi} \int_{0}^{\infty} d y \exp \left\{f\left(y_{\max }\right)+\frac{y^{2}}{2} f^{\prime \prime}\left(y_{\max }\right)\right\}
$$

The exponent $f(y)$ takes its maximum value at the position $y_{\max }=\sqrt{2}\left(-I_{0}\right)^{1 / 4}$. Expanding it around this value in second order and integrating gives:

$$
v \approx \frac{\sqrt{2}}{\pi \tau} e^{-8 / 3\left(-I_{0}\right)^{3 / 2}} \sqrt{-I_{0}}
$$

This approximation is only valid if $f\left(y_{\max }\right)=4 / 3\left(-I_{0}\right)^{3 / 2} \gg 1$ and if $\left|f^{\prime \prime}\left(y_{\max }\right)\right|^{1 / 2} y_{\max }=4\left(-I_{0}\right)^{3 / 4} \gg$ 1.

\subsubsection{High-rate approximation}

For large values of $I_{0}\left(I_{0} \gg 1\right)$, we can approximate the inverse rate again by a Gaussian integral, yielding:

$$
v^{-1} \approx 2 \tau \sqrt{2 \pi} \int_{0}^{\infty} d y \exp \left(-2 I_{0} x^{2}\right)
$$

This recovers the deterministic result,

$$
v=(\pi \tau)^{-1} \sqrt{I_{0}},
$$

from Eq. (3.9). In this limit, the firing rate is independent of the noise strength $\sigma$.

\subsubsection{Medium-rate approximation}

To bridge the high- and low-rate approximation it is possible to expand $v^{-1}$ around $I_{0}=0$ in a series expansion (Colet et al., 1989). This gives:

$$
v^{-1} \approx \tau \sum_{n=0}^{N}(-1)^{n} \frac{B_{n}}{n !} I_{0}^{n}
$$

with

$$
B_{n}=\frac{1}{3}\left(\frac{\pi}{3}\right)^{1 / 2}\left(\frac{9}{2}\right)^{1 / 3} 2^{2 n+1 / 2} \Gamma\left(\frac{2 n+1}{6}\right),
$$

and becomes exact for $N \rightarrow \infty$.

In Fig. 3.7, the stationary density for several values of $I_{0}$ together with the low-, high- and mediumrate approximation of the rate are displayed. While the high-rate approximation works very well close to $I_{0} \gtrsim 0$, the low rate approximation exhibits a maximum at some $I_{0}<0$ and deviates strongly for $I_{0} \lesssim 0$. The medium-rate approximation is shown for $N=4$. Table 3.1 summarizes the solutions in the three regimes. 
A

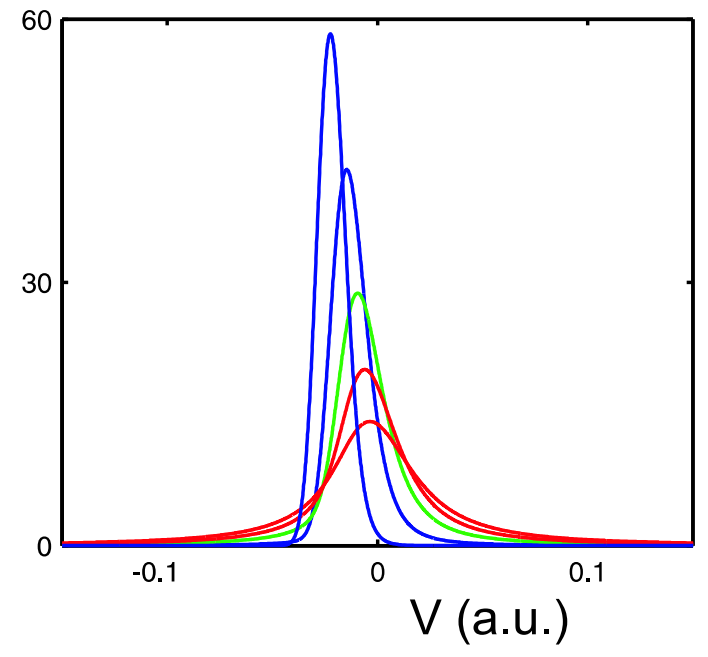

B

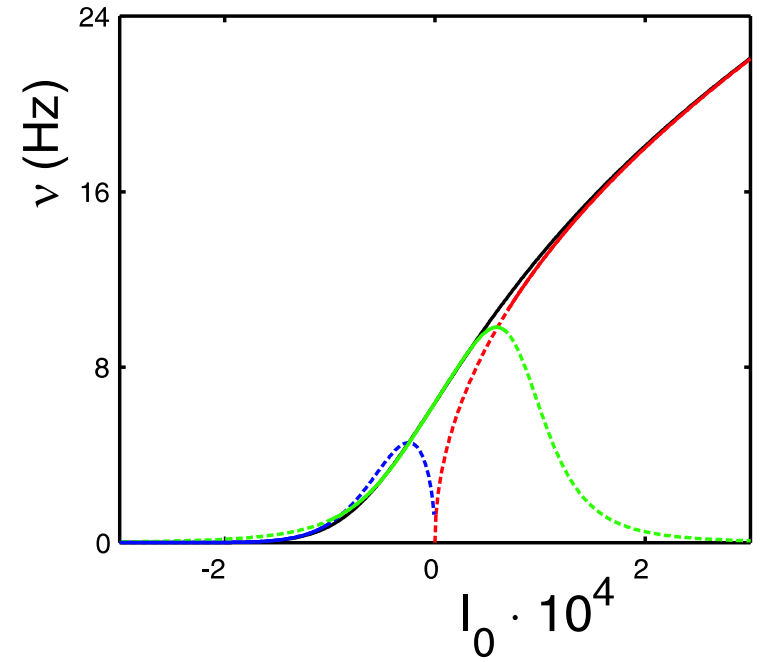

Figure 3.7: (A) Stationary density $P_{\mathrm{st}}(V)$ for several values of $I_{0}$ (red: $I_{0}=[-0.5,-0.2]$, green: $I_{0}=$ 0 , red: $\left.I_{0}=[0.2,0.5]\right)$. (B) Stationary firing $v$ as a function of the input current $I_{0}$ with a fixed noise amplitude $\sigma=10^{-3}$. In blue the low-rate approximation is shown for $I_{0}<0$ and the high-rate approximation in red for $I_{0}>0$. The medium-range approximation is shown in green for $N=4$. The black line denotes the numerical solution of Eq. (3.31). In the regime where the approximations do not apply, the corresponding curve is shown as dashed lines. The different approximations cover the whole range of input current values $I_{0}$ and noise amplitudes $\sigma$.

\begin{tabular}{|c|c|c|}
\hline$I \ll 0$ & $I \approx 0$ & $I \gg 0$ \\
\hline \hline$\frac{\sqrt{2}}{\pi \tau} e^{-8 / 3\left(-I_{0}\right)^{3 / 2}} \sqrt{-I_{0}}$ & $\left(\tau \sum_{n=0}^{N}(-1)^{n} \frac{B_{n}}{n !} !_{0}^{n}\right)^{-1}$ & $(\pi \tau)^{-1} \sqrt{I_{0}}$ \\
\hline
\end{tabular}

Table 3.1: Summary of the three approximations for the stationary rate in different regimes. In the regime $I \approx 0$, the abbreviation $B_{n}=\frac{1}{3}\left(\frac{\pi}{3}\right)^{1 / 2}\left(\frac{9}{2}\right)^{1 / 3} 2^{2 n+1 / 2} \Gamma\left(\frac{2 n+1}{6}\right)$ was used. 


\subsubsection{Coefficient of variation}

As the dynamics of the model is noise-driven, one can not expect that spikes are emitted in a regular fashion, rather will there be some irregularity in the distribution of inter-spike-intervals. An important measure to quantify this irregularity is the coefficient of variation (CV). It is defined as:

$$
C V:=v \Delta T
$$

where $\Delta T$ denotes the standard deviation of the ISI distribution:

$$
\Delta T=\sqrt{\left\langle T^{2}\right\rangle-\langle T\rangle^{2}}
$$

What is expected for the $C V$ in the case of very small or very large input currents? It is intuitively clear that for very small input currents, the emission of an AP can be compared with a Kramer's like activated process. For such a process, the CV is close to unity (Risken, 1996). On the other hand, for very large input currents, the dynamics will be similar to an oscillator which generates APs very regularly. Thus, the $C V$-value is close to zero in this regime. In the regime where the modulus of the input current is comparable to the noise amplitude, the $C V$-value can be expected to lie between these two extreme regimes. I will first compute it in the limit $I_{0}=0$ and then deduce an expansion for small values of $I_{0}$.

The second moment of the ISI distribution is given by (Gardiner, 2004):

$$
\begin{aligned}
\Delta T^{2}= & \frac{16}{\sigma^{2}} \int_{-\infty}^{\infty} d x_{1} \int_{-\infty}^{x_{1}} d x_{2} \int_{-\infty}^{x_{2}} d x_{3} \int_{-\infty}^{x_{3}} d x_{4} \\
& \exp \left\{\frac{2}{\sigma^{2}}\left(V\left(x_{1}\right)+V\left(x_{2}\right)-V\left(x_{3}\right)-V\left(x_{4}\right)\right)\right\}
\end{aligned}
$$

For the standard $\theta$-neuron, $V(x)$ is given by:

$$
V(x)=x^{3} / 3+I_{0} x
$$

In the limit of small input currents, i.e. $I_{0} \ll \sigma$, the four-fold integral in Eq. (3.42) can be solved analytically, yielding:

$$
\Delta T^{2}=\Gamma\left(\frac{1}{3}\right)^{4} / 27\left(\sigma^{2} / 18\right)^{-2 / 3}
$$

Together with Eq. (3.38), this leads to:

$$
C V=\frac{1}{\sqrt{3}} \approx 0.58,
$$

which is, surprisingly, independent of the noise strength $\sigma$.

For $I_{0} \neq 0$ an expansion for small $k=3 I_{0}\left(4 /\left(3 \sigma^{2}\right)\right)^{2 / 3} \ll 1$ gives:

$$
\Delta T^{2}=\left(\sigma^{2} / 18\right)^{-2 / 3} \sum_{n=0}^{N}(-1)^{n} k^{n} \tilde{B}_{n} / n !
$$


with

$$
\begin{aligned}
\tilde{B}_{n}= & \int_{-\infty}^{\infty} d x_{1} \int_{-\infty}^{x_{1}} d x_{2} \int_{-\infty}^{x_{2}} d x_{3} \int_{-\infty}^{x_{3}} d x_{4} \\
& \left(x_{1}+x_{2}-x_{3}-x_{4}\right)^{n} \exp \left(x_{4}^{3}+x_{3}^{3}-x_{2}^{3}-x_{1}^{3}\right) .
\end{aligned}
$$

Together with Eq. (3.38), this yields in linear order, i.e. for small $|k| \ll 1$, to,

$$
C V=\alpha+\beta v
$$

with $\alpha \approx 0.92$ and $\beta \approx-0.1435 \sigma^{2 / 3}$. This result shows that the $\mathrm{CV}$ increases with decreasing values of the stationary rate. Moreover it provides an important scaling relation for the noise amplitude. One has to keep in mind, however, that the rate and the noise amplitude are not independent. An increase in the noise amplitude will inevitably lead to an increase of the firing rate.

\subsection{Dynamic responses}

In the following, I discuss the dynamic response properties of the generalized $\theta$-neuron. Unlike previous studies which derived the dynamic response properties for IF models, a novel method is introduced, which is based on expanding the Fokker-Planck operator $\hat{L}$ in Eq. (3.14) into a complete orthonormal basis set. Using the resulting matrix representation, I compute the dynamic response properties to a very high accuracy which can hardly be met by direct numerical simulations. This method works in the presence of both white noise and correlated noise inputs for the standard and the generalized $\theta$-neuron model. In addition, using a highly efficient numerical method, this method is at the same time much faster than previously proposed direct simulation techniques.

\subsubsection{General framework}

For an arbitrary time-varying input, the Fokker-Planck operator $\hat{L}(\theta, z, t)$ can always be split into two parts:

$$
\hat{L}(\theta, z, t)=\hat{L}_{0}(\theta, z)+\varepsilon \hat{L}_{1}(\theta, z, t),
$$

where $\hat{L}_{0}(\theta, z)$ is the former time-independent Fokker-Planck operator and $\hat{L}_{1}(\theta, z, t)$ takes all timedependencies of the external input. In the following I require that the time-dependent inputs are small in magnitude, i.e. $\varepsilon \ll 1$.

The time-dependent solution $P_{\mathrm{TD}}(\theta, z, t)$ can then be expanded in powers of $\varepsilon$ around the stationary pdf $P_{0}(\theta, z)$ of the operator $\hat{L}(\theta, z)$ :

$$
P_{\mathrm{TD}}(\theta, z, t)=P_{0}(\theta, z)+\varepsilon \tilde{P}(\theta, z, t)+\mathscr{O}\left(\varepsilon^{2}\right)
$$

This series expansion can now be inserted into the Fokker-Planck equation. In linear order in $\varepsilon$ this yields a dynamical equation for the function $\tilde{P}(\theta, z, t)$ :

$$
\partial_{t} \tilde{P}(\theta, z, t)=\hat{L}_{0}(\theta, z) \tilde{P}(\theta, z, t)+\hat{L}_{1}(\theta, z, t) P_{0}(\theta, z)
$$


As this is a linear equation, it can readily be solved. The formal solution is given by:

$$
\tilde{P}(\theta, z, t)=\int_{-\infty}^{t} e^{\hat{L}_{0}(\theta, z)\left(t-t^{\prime}\right)} \hat{L}_{1}(\theta, z, t) P_{0}(\theta, z) d t^{\prime}
$$

In the following, I will consider time-dependent stimuli of the form:

$$
\hat{L}_{1}(\theta, z, t)=e^{i \omega t} \hat{L}_{1}(\theta, z)+c . c .
$$

The solution of Eq. (3.52) is then given by:

$$
\tilde{P}(\theta, z, t)=\sum_{k} \frac{c_{k}}{i \omega-\lambda_{k}} P_{k}(\theta, z) e^{i \omega t}+c . c .
$$

The $c_{k}$ are the expansion coefficients of $\hat{L}_{1}(\theta, z) P_{0}(\theta, z)$ into the eigenfunctions $P_{k}(\theta, z)$ of $\hat{L}_{0}(\theta, z)$. It will be shown in Sec. 3.9.1, how the eigenfunctions and eigenvalues of the operator $\hat{L}_{0}$ are computed efficiently. The time-dependent firing rate is given by Eq. (3.25):

$$
\begin{aligned}
v(t)= & \tau^{-1} \int_{-\infty}^{\infty} d z\left\{\left(1-\cos \theta_{s}\right)+\left(1+\cos \theta_{s}\right)\left(I_{0}+\sigma z+\alpha\left(1+\tanh \left(\beta \tan \left(\theta_{s} / 2\right)\right)\right)\right)\right\} \\
& \cdot\left(P_{0}\left(\theta_{s}, z\right)+\varepsilon \tilde{P}\left(\theta_{s}, z, t\right)\right) \\
=: & v_{0}+\varepsilon v_{1}(\omega) e^{i(\omega t+\varphi(\omega))}+\text { c.c. }
\end{aligned}
$$

In the linear response regime, the time-dependent firing rate responds with the same frequency as the input modulation. Thus, it is possible to construct any input just by linear superposition:

$$
\hat{L}_{0}+\varepsilon \int d \omega f(\omega) \hat{L}_{1}(\theta, z) e^{i \omega t} \longrightarrow v_{0}+\varepsilon \int d \omega f(\omega) v_{1}(\omega) e^{i(\omega t+\varphi(\omega))}
$$

This means that the theory presented above can describe the response to arbitrary input stimuli, provided that the input modulations are small in magnitude.

While the analysis presented above provides the framework for studying the response to all kind of stimulations, which are small in amplitude, i.e. arbitrary $\hat{L}_{1}(\theta, z)$ we will consider in the following two paradigmatic types of external stimulations:

1. Modulations in the mean input current:

$$
I_{0} \longrightarrow I_{0}+\varepsilon e^{i \omega t}
$$

2. Modulations in the noise amplitude:

$$
\sigma \longrightarrow \sigma+\varepsilon e^{i \omega t}
$$

Inserting these into the Fokker-Planck operator $\hat{L}$, gives different resulting forms of the operator $\hat{L}_{1}$. They are summarized in table 3.2 both for white noise stimulation and correlated input noise. 


\begin{tabular}{|c|c|c|}
\hline & Mean current modulation & Noise amplitude modulation \\
\hline White noise input & $-\tau^{-1} \partial_{\theta}(1+\cos \theta)$ & $\sigma \tau^{-1} \partial_{\theta}(1+\cos \theta) \partial_{\theta}(1+\cos \theta)$ \\
\hline Colored noise input & $-\tau^{-1} \partial_{\theta}(1+\cos \theta)$ & $-\tau^{-1} z \partial_{\theta}(1+\cos \theta)$ \\
\hline
\end{tabular}

Table 3.2: Different forms of the operator $\hat{L}_{1}$ for either a white or correlated input noise current and two types of input modulations. The operator is the same in both cases for modulations in the mean input current, while for modulations in the noise amplitude, $\hat{L}_{1}$ explicitly includes the second dynamical variable $z(t)$.

\subsection{Universality of the high frequency behavior}

In the introduction, I briefly discussed several studies which showed that in the high frequency limit the response modulation does not go to zero for the class of IF models in a realistic parameter regime. I will therefore also derive the high-frequency limit for the generalized $\theta$-neuron model. In the previous subsection, the problem of computing the transmission function $v_{1}(\omega)$, was reduced to an eigenvalue problem of the operator $\hat{L}_{0}$, which could only be solved numerically. In the limit $\omega \rightarrow \infty$, however, the asymptotic decay of $v_{1}(\omega)$ can be calculated analytically. Inserting Eq. (3.54) into Eq. (3.51) leads to:

$$
\left(i \omega-\hat{L}_{0}\right) \tilde{P}(\theta, z, t) e^{-i \omega t}=\hat{L}_{1} P_{0}(\theta, z)
$$

\subsubsection{Dynamics insensitive at $\operatorname{AP}\left(\theta_{s}=\pi\right)$}

I will first discuss the case in which a spike is emitted at the phase $\theta_{s}=\pi$. There, the dynamics is insensitive to external inputs and symmetric, i.e. $\dot{\theta}(\pi-\delta)=\dot{\theta}(\pi+\delta)$. In this case, the modulus of the right hand side vanishes at $\theta_{s}=\pi$ for all operators $\hat{L}_{1}(\theta, z)$ from table 3.2. Therefore, the $\tilde{P}(\theta, z, t)$ has to be at least of order $\omega^{-2}$, such that the left hand side vanishes for $\omega \rightarrow \infty$. Subsequent differentiation of Eq. (3.51) and reinsertion leads to:

$$
\left(\omega^{2}+\hat{L}_{0}\right) \tilde{P}(\theta, z, t)=-\hat{L}_{0} \hat{L}_{1} P_{0}(\theta, z)
$$

The right hand side does not vanish at $\theta=\pi$ in the case of a mean current modulation for both types of noise inputs and in the case of a modulation in the noise amplitude in the presence of a correlated input current. Since both sides have to be real valued, the modulus of $\tilde{P}(\theta, z)$ has to be $\propto \omega^{-2}$ and the phase $\varphi(\omega)$ goes to $-\pi$.

For the case of a white noise input and a modulation in the noise amplitude $\sigma$, the right hand side of Eq. (3.58) is zero, which requires an expansion up to third order in $\omega$. It then turns out, using the same argument as above, that the modulus $\tilde{P}(\theta)$ even decays as $\omega^{-3}$ and the relative phase goes to $-3 \pi / 2$.

One would of course expect that there is a cross-over from the white-noise regime to the correlated noise regime for small values of the correlation time $\tau_{c}$. Indeed, this cross-over from the $\omega^{-3}$ to the $\omega^{-2}$ behavior can be observed and will be studied in Sec. 3.10.1.

\subsubsection{The generic case $\left(\theta_{s} \neq \pi\right)$}

For $\theta_{s}=\pi-\delta, \delta>0$ the right hand side of Eq. (3.57) does not vanish. This means that for large frequencies the rate modulation $v_{1}(\omega)$ decays as $\omega^{-1}$ and the relative phase shift $\varphi(\omega)$ is $-\pi / 2$. 


\begin{tabular}{|c||c|c|c|c||c|c||c|c|}
\hline \multicolumn{1}{|c||}{} & \multicolumn{3}{c||}{ Generalized $\theta$-neuron } & \multicolumn{2}{c||}{ LIF model } & \multicolumn{2}{c|}{ CB models } \\
\hline \hline & \multicolumn{2}{|c||}{$\theta_{s}=\pi$} & \multicolumn{2}{c||}{$\theta_{s} \neq \pi$} & \multicolumn{2}{c|}{ Fixed Threshold } & \multicolumn{2}{c|}{ Limit cycle } \\
\hline Noise correlation & $\tau_{c}>0$ & $\tau_{c} \rightarrow 0$ & $\tau_{c}>0$ & $\tau_{c} \rightarrow 0$ & $\tau_{c}>0$ & $\tau_{c} \rightarrow 0$ & $\tau_{c}>0$ & $\tau_{c} \rightarrow 0$ \\
\hline \hline Mean modulation & $\omega^{-2}$ & $\omega^{-2}$ & $\omega^{-1}$ & $\omega^{-1}$ & $\omega^{0}$ & $\omega^{-1 / 2}$ & $\omega^{-1}$ & $\omega^{-1}$ \\
\hline Noise modulation & $\omega^{-2}$ & $\omega^{-3}$ & $\omega^{-1}$ & $\omega^{-1}$ & $\omega^{0}$ & $\omega^{0}$ & $\omega^{-1}$ & $\omega^{-1}$ \\
\hline
\end{tabular}

Table 3.3: High frequency behavior of the generalized $\theta$-neuron, the leaky integrate-and-fire model and conductance-based models. The response of the LIF model was derived in (Brunel et al., 2001; Lindner \& Schimansky-Geier, 2001), the response of a conductance-based model for $\tau_{c}>0$ and a mean current modulation in (Fourcaud-Trocmé et al., 2003). The asymptotic response of the conductance-based model in the other cases follows from the same argument as for the asymptotic response of the $\theta$-neuron, and is confirmed by direct numerical simulations (data not shown).

The response of the conductance-based model is the generic behavior of any dynamical system which does not have any special symmetries or boundary conditions which suppress the first order expansion in $1 / \omega$.

This demonstrates that the $\theta$-neuron, in contrast to IF models, captures a key property of the dynamic response of more complex neuron models. In table 3.3, the high frequency behavior of the generalized $\theta$-neuron is summarized and compared with the high-frequency behavior of conductance-based model neurons as well as the classical LIF model.

I would like to point out that the $\omega^{-2}$ and $\omega^{-3}$ decay of the $\theta$-neuron is only due to (i) the insensitivity of the dynamics to inputs at $\theta=\pi$ and the symmetric up- and downstroke of the AP around $\theta_{s}=\pi$. Here, both conditions are lifted by defining the spike phase at a different value than $\pi$. Another way to induce a $\omega^{-1}$-decay would be to change the right hand side of Eq. (3.12), such that $\hat{L}_{1} P_{0}$ does not vanish at $\theta=\pi$. This could for instance be achieved by introducing high order terms in $\cos \theta$, which would result in a structural change of the oscillator dynamics.

For small values of $\delta$ and intermediate frequencies, a cross-over between the $\omega^{-2}$ and $\omega^{-1}$ asymptotic decay can be observed. To show this cross-over, it is, however, necessary to calculate the linear response function $v_{1}(\omega)$ for arbitrary frequencies.

A very important point to mention here is that the asymptotic decay of the rate-response function is completely independent of the AP onset parameters $\alpha$ and $\beta$ of the generalized $\theta$-neuron. This already indicates that the high-frequency limit may not be a good criterion to decide, if a given neuron model matches the dynamics of real neurons. The second surprising result is the high-frequency limit in the presence of a white noise input. For the class of IF models, the transmission function decays $\propto 1 / \sqrt{\omega}$ for a modulation in the mean input current, while it does not decay for a modulation of the noise amplitude. The generalized $\theta$-neuron on the other hand seems to exhibit exactly the opposite behavior, namely a faster decay for modulations in $\sigma$, which is even $\propto \omega^{-3}$ and a slower decay $\propto \omega^{-2}$ for modulations in the mean input current, which even turns out to be dependent on the phase $\theta_{s}$ at which a spike is emitted.

These surprising results clearly demonstrate that it is crucial to have a method at hand to determine the full response function with a high accuracy. Only by using this method, will it be possible to show what the typical response properties of the generalized $\theta$-neuron are and to investigate the dependence on the AP onset dynamics. 


\subsection{A sparse matrix representation of the Fokker-Planck operator}

As evident from Eq. (3.54), the linear response properties are complete determined by the spectrum and eigenfunctions of the Fokker-Planck operator $\hat{L}$. In this section, I present a semi-analytical approach to compute the stationary, as well as the dynamic response properties in the presence of either a white or a temporally correlated noise current. This approach allows for the computation of the response properties with a very high accuracy without any approximations.

The principle approach is to expand $\hat{L}$ into a complete orthonormal basis leading to a sparse matrix representation for which eigenvalues and eigenfunctions can be computed numerically. This procedure is well-known and described e.g. in (Risken, 1996). There are, however, two major subtleties which render this problem non-trivial:

1. The resulting matrix is very large in the parameter regime we are interested in (up to $10^{6} \times 10^{6}$ )

2. The operator $\hat{L}$ is not Hermitian, and thus standard diagonalization procedures such as the Lanczos algorithm can not be applied.

The first problem is solved by using a basis-set which results in a very sparse matrix representation. This matrix representation has the property that the number of non-zero entries scales linearly with the number of basis functions. I will then use a high performance iterative scheme, the Arnoldi method (Lehoucq et al., 1998), to compute the eigenfunctions and the spectrum of this matrix, which solves the second problem.

The basis set for the case of Gaussian white noise input and in the case of a correlated noise input will naturally be different, as will be the matrix representation of the operator $\hat{L}$. In principle, it would be sufficient to consider only the case of correlated noise inputs and then take the limit $\tau_{c} \rightarrow 0$. Nevertheless, I will consider the case of a Gaussian white noise input explicitely and separately from the case of a correlated input current. I do this for two reasons:

1. The computation of eigenvalues and eigenfunctions for a white noise input is not as computationally time-consuming because the required basis set and thus the resulting matrix representation is considerably smaller than in the case of a correlated input current.

2. The white noise case serves as an independent check for the spectrum and the eigenfunctions of the case with a correlated noise input in the limit $\tau_{c} \rightarrow 0$.

\subsubsection{Eigenvalues and eigenfunctions for a correlated noise input}

In a first step, the pdf $P(\theta, z, t)$ is replaced in an eigenmode Ansatz with $e^{\lambda_{k} t} P_{k}(\theta, z)$. By inserting this Ansatz into Eq. (3.14), the exponential prefactor cancels out yielding,

$$
\lambda_{k} P_{k}(\theta, z)=\hat{L}_{0} P_{k}(\theta, z) .
$$

The time-dependent solution, i.e. the relaxation to the stationary density, can always be given in terms of eigenfunctions and eigenvalues (Risken, 1996):

$$
P(\theta, z, t)=e^{\hat{L}_{0}\left(t-t_{0}\right)} P_{\text {initial }}(\theta, z)=\sum_{k} a_{k} e^{\lambda_{k}\left(t-t_{0}\right)} P_{k}(\theta, z)
$$


where the initial distribution $P_{\text {initial }}$ was decomposed into eigenfunctions $P_{k}(\theta, z)$ :

$$
P_{\text {initial }}(\theta, z)=\sum_{k} a_{k} P_{k}(\theta, z)
$$

\subsubsection{Properties of eigenvalues and eigenfunctions}

It can be shown that due to the imposed boundary conditions, the set $\left\{\lambda_{k}\right\}$, i.e. the spectrum of $\hat{L}(\theta, z)$, is discrete (Gardiner, 2004). It is important to note that there is a macroscopic drift in the system, which means that detailed balance is not fulfilled. Thus, the operator $\hat{L}$ is not Hermitian (Gardiner, 2004). Consequently, the resulting spectrum $\left\{\lambda_{k}\right\}$ and the corresponding eigenfunctions $P_{k}(\theta, z)$ are complex and the eigenfunctions $P_{k}(\theta, z)$ of $\hat{L}_{0}$ are not orthogonal. Nevertheless, the eigenfunctions form a basis.

By complex conjugation of Eq. (3.59), it is easy to show that to every eigenvalue $\lambda_{k}$ with corresponding eigenfunction $P_{k}(\theta, z)$, there exists an eigenvalue $\lambda_{k}^{*}$ with eigenfunction $P_{k}^{*}(\theta, z)$. This guarantees that a real solution can always be constructed. Moreover, it can be shown that a solution with eigenvalue $\lambda=0$ always exists. As apparent from Eq. (3.60), this solution corresponds to the stationary density.

Because $P(\theta, z, t)$ is a pdf, it has to be normalized to unity at all times. Therefore, according to Eq. (3.60), the mean value of all eigenfunctions except $P_{0}(\theta, z)$ has to vanish:

$$
\int_{-\pi}^{\pi} d \theta \int_{-\infty}^{\infty} d z P_{k}(\theta, z)=0
$$

In the following, the eigenvalues will be sorted according to their real part:

$$
0=\lambda_{0}>\operatorname{Re} \lambda_{1}>\cdots>\operatorname{Re} \lambda_{k}>\cdots
$$

\subsubsection{Matrix equation}

To actually compute the spectrum and eigenfunctions I expand the pdf $P(\theta, z)$ into a set of complete orthonormal functions:

$$
P(\theta, z)=\sum_{m=0}^{\infty} a_{n, m} \psi_{n, m}(\theta, z)
$$

which is a linear sum over basis functions $\psi_{n, m}(\theta, z)$. They are defined as:

$$
\psi_{n, m}(\theta, z)=\left(2^{m+1} \sqrt{\pi \tau / 2 \tau_{c}} m !\right)^{-1 / 2} e^{i n \theta} H_{m}\left(\sqrt{2 \tau_{c} / \tau} z\right) e^{-z^{2} \tau_{c} / \tau}
$$

In the $\theta$-directions, they consist of plane waves, while in the $z$-direction harmonic-oscillator functions were used with the Hermite polynomials $H_{m}(z)$ (Abramowitz \& Stegun, 1972). This expansion obeys the imposed boundary conditions.

To derive a matrix representation of $\hat{L}_{0}$, Eq. (3.64) is inserted into Eq. (3.14). Multiplying from left with $\psi_{n^{\prime}, m^{\prime}}^{*}(\theta, z)$ and integrating over the whole domain leads to a matrix eigenvalue equation for the 
$\left(a_{n, m}\right)$ :

$$
\begin{aligned}
\lambda a_{n, m}= & \left(-i \tau^{-1}\left(1+I_{0}\right) n-\tau_{c}^{-1} m\right) a_{n, m} \\
& +(2 \tau)^{-1} i\left(1-I_{0}\right) n\left(a_{n-1, m}+a_{n+1, m}\right) \\
& -\frac{i n \sigma}{2 \sqrt{\tau \tau_{c}}}\left((m+1) a_{n, m+1}+m a_{n, m-1}\right. \\
& +\frac{1}{2}(m+1)\left(a_{n-1, m+1}+a_{n+1, m+1}\right) \\
& \left.+\frac{1}{2}(m+1)\left(a_{n-1, m-1}+a_{n+1, m-1}\right)\right) \\
+ & \left(\sqrt{2 \tau \tau_{c}}\right)^{-1} \sqrt{(m+1)(m+2)} a_{n, m+2} \\
& -i \tau^{-1} \alpha\left(a_{n, m} c_{0}+\frac{1}{2} \sum_{k=1}^{K}\left(i c_{k}+s_{k}\right) a_{n-k, m}+\left(i c_{k}-s_{k}\right) a_{n+k, m}\right) \\
= & \sum_{n^{\prime}, m^{\prime}} L_{n, m ; n^{\prime}, m^{\prime}} a_{n^{\prime}, m^{\prime}}=\mathbf{L} \vec{a} .
\end{aligned}
$$

The coefficients $c_{k}$ and $s_{k}$ denote the Fourier components of $(1+\cos \theta) \tanh (\beta \tan (\theta / 2))$ of the expansion in $\cos (k \theta)$ and $\sin (k \theta)$ up to order $K$, respectively.

To solve this eigenvalue problem numerically, the indices $n$ and $m$ have to be restricted:

$$
-N \leq n \leq N, \quad 0 \leq m \leq M
$$

In all three regimes, the stationary pdf can be expected to be very peaked around the stable fixed point. This implies, that many plane wave basis functions, i.e. up to $N \approx 10^{4}$ will be needed. With $M=50$ the matrix that we have to diagonalize will be of size $10^{6} \times 10^{6}$. To represent this matrix in full form would require $3.8 \cdot 10^{3} \mathrm{~GB}$ of storage capacity, which is well beyond the capacity of even large computer clusters.

With the chosen basis set, it turns out, however, that the matrix $\mathbf{L}$ in Eq. (3.66) is very sparse. For the $\theta$-neuron, i.e. $\alpha=0$, it even connects an element $a_{n, m}$ to the elements $a_{n \pm 1, m \pm 1}$ and $a_{n, m+2}$ only. For $\alpha>0$ the number of nonzero entries in $\mathbf{L}$ only depends on the number of Fourier components $K$ of the AP onset term of the generalized model.

Generically, however, the number of elements in the matrix $\mathbf{L}$ is only of order $N \times M$, i.e. very sparse compared to its full size $N^{2} \times M^{2}$ (Fig. 3.8 shows a sketch of the matrix $\mathbf{L}$ ). This makes it possible to use a high performance iterative algorithm, the Arnoldi-method (Trefethen \& Bau, 1997; Lehoucq et al., 1998), to solve this eigenvalue problem numerically.

The spectrum and stationary pdf are shown in Fig. 3.9 for $\tau_{c}=10 \mathrm{~ms}$ and a stationary rate of $v=$ $10 \mathrm{~Hz}$. The spectrum is composed of several wedges which are located at integer multiples of $\tau_{c}^{-1}$. Close to the tip, the real parts of the eigenvalues in each wedge are first approximately equidistant and grow approximately quadratically for larger excitations. The imaginary parts are approximately equidistant. According to the time evolution in Eq. (3.60), the real part of the eigenvalues determines the decay rate of their corresponding eigenfunction, while their imaginary part determines their oscillating frequency. In Fig. 3.10, several spectra for different values of the AP onset speed $\alpha$ are displayed. For increasing values of $\alpha$, the imaginary parts of the eigenvalues increase, while the real parts decrease, reflecting a smaller relaxation time constant. 


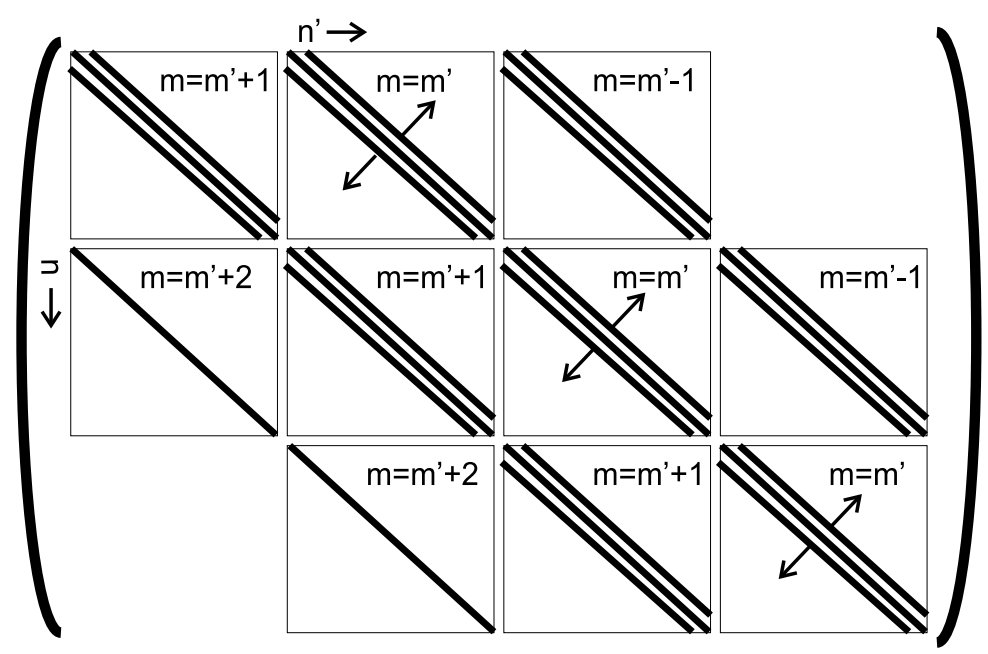

Figure 3.8: Structure of the sparse matrix representation Eq. (3.66) of the Fokker-Planck operator $\hat{L}$, resulting from an expansion into the basis, given by Eq. (3.65).
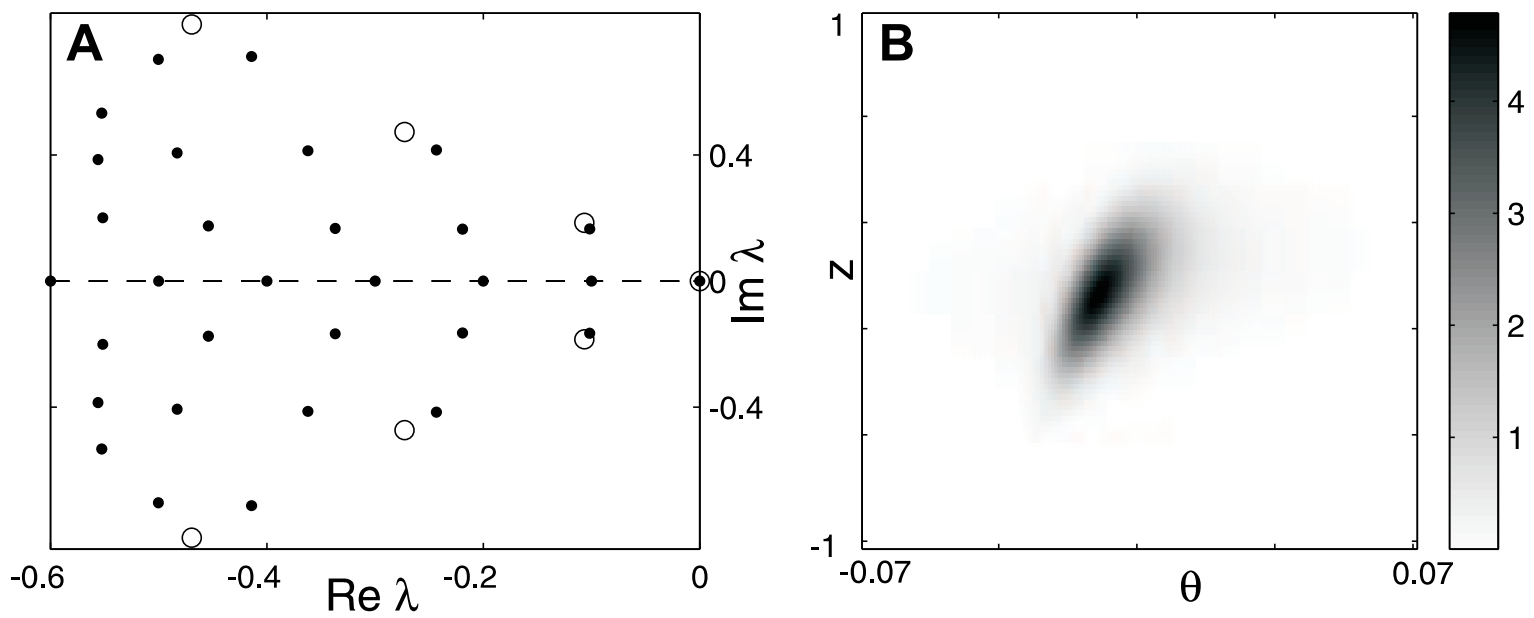

Figure 3.9: (A) Eigenvalues of the operator $\hat{L}_{0}$, filled circles denote eigenvalues for the case of a correlated input noise, open circles are the eigenvalues for the white noise case. The first 27 eigenvalues are shown. The spectrum is decomposed into wedges where the tip of each wedge is located at integer multiples of $\tau_{c}^{-1}$. The real part of an eigenvalue $\lambda_{k}$ determines the damping, the imaginary part the oscillation frequency of a mode $P_{k}(\theta, z)$. (B) Stationary pdf. The pdf is peaked around at 0 and slightly asymmetric, showing that $\theta$ is correlated with the input noise $z$.

Parameters are $\tau_{c}=10 \mathrm{~ms}, I_{0}=0, \alpha=0$ and $v=20 \mathrm{~Hz}$. For the numerical diagonalization, the basis was restricted to $N=5000$ and $M=30$. 
The rate is given by Eq. (3.24) (Here only shown for $\theta_{s}=\pi$ ):

$$
v(t)=\tau^{-1} \sum_{n, m} a_{n, m}^{0} \int_{-\infty}^{\infty} \psi_{n, m}\left(\theta_{s}, z\right) d z,
$$

with the expansion coefficients $a_{n, m}^{0}$ of the stationary rate.

Although a high-performance iterative algorithm was used to compute the spectrum and eigenfunctions, I would like to point out that the computation of the spectrum and eigenfunctions can be very demanding, especially for correlated input currents and $\alpha>0$.

To compute for instance the linear response properties in Sec. 3.10.2, I had to use a very large basis set, requiring up to 8GB of memory capacity. The big advantage is, however, that the spectrum and eigenfunctions can be, once calculated, stored on hard drive and used to calculate the response to different forms of time-varying inputs.

In Sec. 3.9.5, I will present an alternative method which can be used to compute the stationary density and rate without having to solve the full eigenvalue problem.

\subsubsection{Eigenvalues and eigenfunctions for a white noise input}

The Fokker-Planck equation which describes the dynamics of the probability density function is given by Eq. (3.22). The eigenfunctions $P_{k}(\theta)$ of the operator $\hat{L}_{\mathrm{GWN}}$ are expanded into plane waves:

$$
P_{k}^{\mathrm{GWN}}(\theta)=\sum_{n=-\infty}^{\infty} b_{n}^{(k)} e^{i n \theta}
$$

Naturally, the eigenfunctions $P_{k}^{\mathrm{GWN}}$ fulfill the same properties as in the case of a correlated noise input discussed in the previous section.

The corresponding eigenvalue equation for the coefficients $b_{n}$ is derived similarly as for $\tau_{c}>0$. It is given by:

$$
\begin{aligned}
& \lambda b_{n}=\frac{1}{4 \tau}\{-n\left(4 i\left(I_{0}+1\right)+3 n \sigma^{2}\right) b_{n} \\
&+n\left(2 i\left(1-I_{0}\right)+(1-2 n) \sigma^{2}\right) b_{n-1} \\
&+n\left(2 i\left(1-I_{0}\right)-(1+2 n) \sigma^{2}\right) b_{n+1} \\
&+\frac{1}{2}(n-1) n \sigma^{2} b_{n-2} \\
&\left.-\frac{1}{2}(n+1) n \sigma^{2} b_{n+2}\right\} \\
&-i \tau^{-1} \alpha\left(a_{n, m} c_{0}+\frac{1}{2} \sum_{k=1}^{K}\left(i c_{k}+s_{k}\right) a_{n-k, m}+\left(i c_{k}-s_{k}\right) a_{n+k, m}\right) \\
&=\sum_{n^{\prime}, m^{\prime}} L_{n, m ; n^{\prime}, m^{\prime}} a_{n^{\prime}, m^{\prime}}=\mathbf{L}^{\mathrm{GWN}} \vec{a} .
\end{aligned}
$$

To compute eigenvalues and eigenfunctions numerically, the basis set has to be restricted again:

$$
-N \leq n \leq N
$$


In this limit, the stationary firing rate is calculated in a straightforward fashion from Eq. (3.27) (here only for $\theta_{s}=\pi$ ):

$$
v^{\mathrm{GWN}}=2 \tau^{-1} P^{\mathrm{GWN}}\left(\theta_{s}\right)=2 \tau^{-1} \sum_{n=-N}^{N}(-1)^{n} b_{n}
$$

\subsubsection{Efficient computation of the stationary density and rate}

For network simulations, it is crucial to be able to compute the stationary density and rate efficiently. It turns out that the sparse matrix representation offers an easy and computationally efficient way to do this without having to compute the spectrum and eigenfunctions.

As seen in the previous section, the spectrum of the Fokker-Planck operator of the $\theta$-neuron is always discrete with one eigenvalue at 0 and with all other values having a negative real part. The stationary density can thus be computed in the following way:

$$
\begin{aligned}
& \lim _{n \rightarrow \infty}\left(\frac{1}{\mathbf{L}-\mathbf{1}}\right)^{n}(-1)^{n} P_{\text {random }}(\theta, z) \\
& =P_{0}(\theta, z)+\lim _{n \rightarrow \infty} \sum_{k>0} a_{k}\left(\lambda_{k}-1\right)^{-n}(-1)^{n} P_{k}(\theta, z)=P_{0}(\theta, z)
\end{aligned}
$$

Here $P_{\text {random }}(\theta, z)$ is some random function with $\int_{-\infty}^{\infty} P_{\text {random }}(\theta, z) d \theta d z=1$ and the $a_{k}$ are the expansion coefficients of $P_{\text {random }}(\theta, z)$ into eigenfunctions of $\hat{L}$. Since all eigenvalues $\lambda_{k}$ for $k>0$ are smaller than zero, the second term on the right hand side will go to zero. This leaves us with the problem of computing $(\mathbf{L}-1)^{-n}$. With the sparse matrix representation $\mathbf{L}$ from Eq. (3.66), this can be done very efficiently by using the following scheme:

1. Compute the LU-decomposition of $\mathbf{L}-\mathbf{1}$ :

$$
\mathbf{L}-\mathbf{1}=\mathbf{A} \cdot \mathbf{B} .
$$

2. Choose some random initial starting vector $b^{(0)}$.

3. Solve the linear systems $\mathbf{A} y=b^{(k)}$ and $\mathbf{B} b^{(k+1)}=b^{(k)}$.

4. Reiterate step 3 until $b^{(k)}$ converges with desired accuracy to the stationary density.

5. Normalize the stationary density using $\int d \theta d z P_{0}(\theta, z)=\sum_{n, m} b_{n, m}^{(k)} \int d \theta d z \psi_{n, m}(\theta, z) \stackrel{!}{=} 1$.

6. The stationary rate is then given by Eq. (3.68).

In Fig. 3.12, the convergence of the rate using the scheme above is exemplified. After only a few iterations, the rate and the density converges, after which the scheme can be aborted. In Fig. 3.11, the stationary rate is shown as a function of the input correlation time $\tau_{c}$ in the critical regime $I_{0}=0$ and in the subthreshold regime $I_{0}=-0.1$. In the critical regime, an increase in the input correlation only slightly influences the firing rate, while in the subthreshold regime already a small increase in the input correlation leads to a substantial reduction of the rate compared to white noise input. 


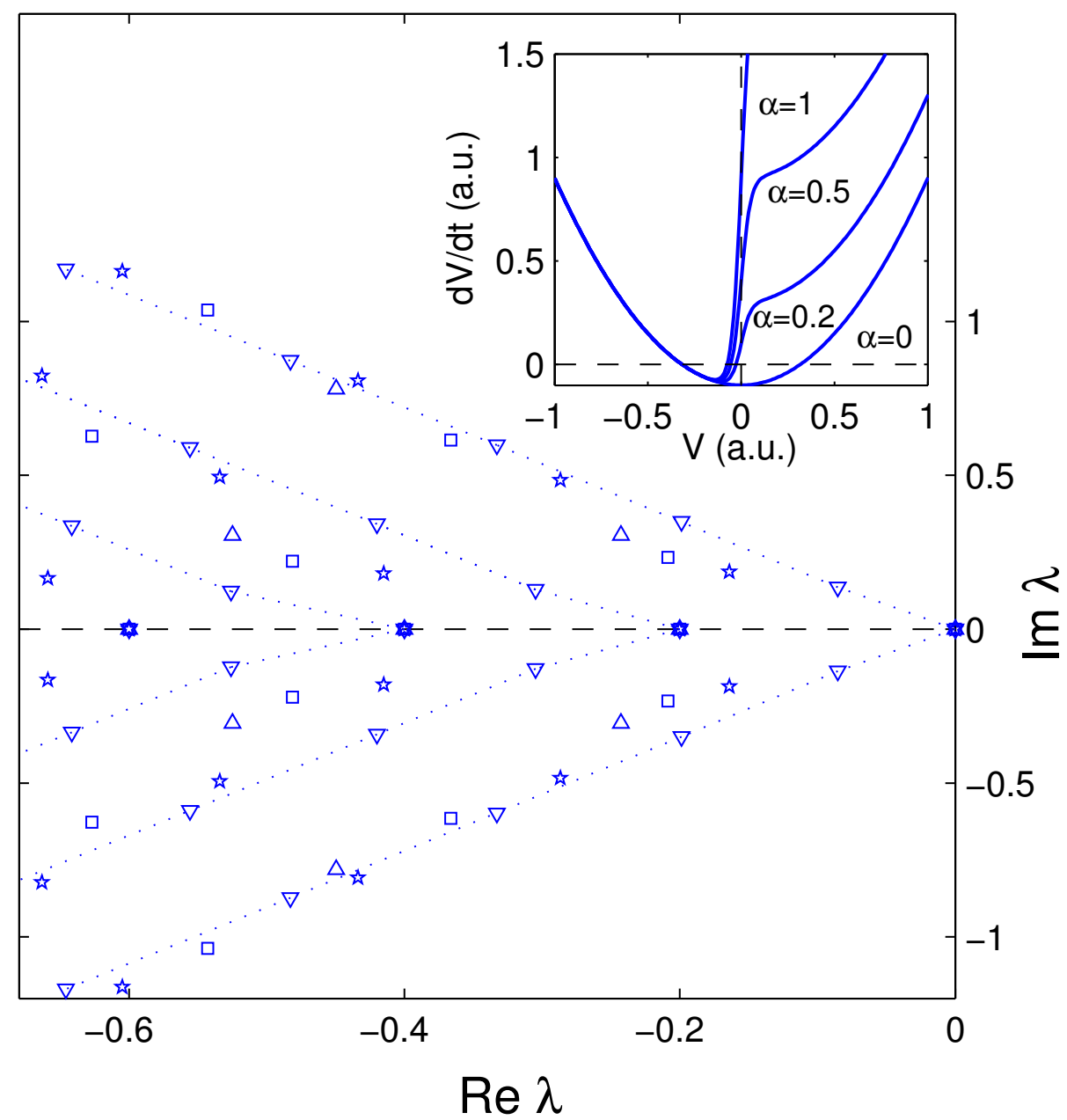

Figure 3.10: Eigenvalues of the operator $\hat{L}_{0}$ for different values of the AP onset speed. $(\alpha=0(\nabla)$, $\alpha=0.2(\star), \alpha=0.5(\square)$ and $\alpha=1.0(\triangle))$. The other parameters are $I_{0}=-0.1, \beta=20$ and $\tau_{c}=5 \mathrm{~ms}$. The noise strength $\sigma$ has been chosen to achieve a stationary rate of $30 \mathrm{~Hz}$. The dotted lines connecting the eigenvalues for $\alpha=0$ have been added as a guide for the eye.

For increasing values of $\alpha$, the imaginary parts of the eigenvalues increase, while the real parts move to smaller values, indicating that for larger values of $\alpha$ the relaxation time of the MP decreases. 


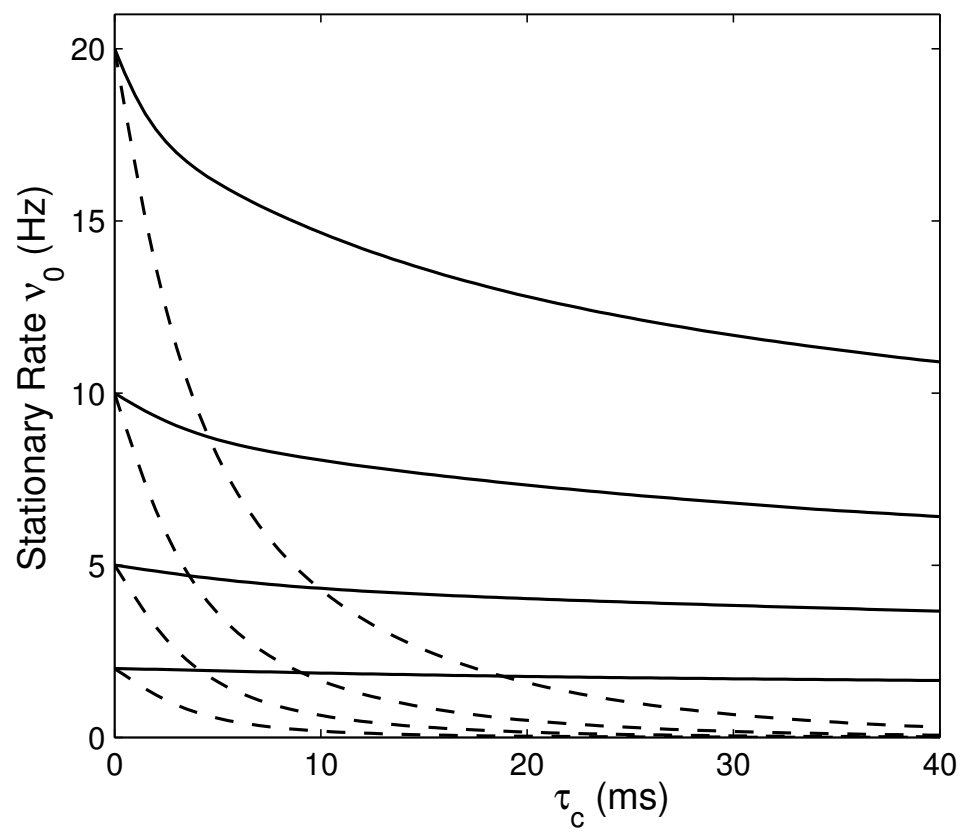

Figure 3.11: Stationary rate for different values for $v\left(\tau_{c}=0\right)$ and $I_{0}$ as a function of $\tau_{c}$. For increasing values of $\tau_{c}$ the stationary rate decreases. Shown are eight curves for all combinations of $v\left(\tau_{c}=\right.$ $0) \in\{2,5,10,20 \mathrm{~Hz}\}$ and $I_{0} \in\{-0.1$ (dashed), 0 (solid) $\}, \alpha=0$.

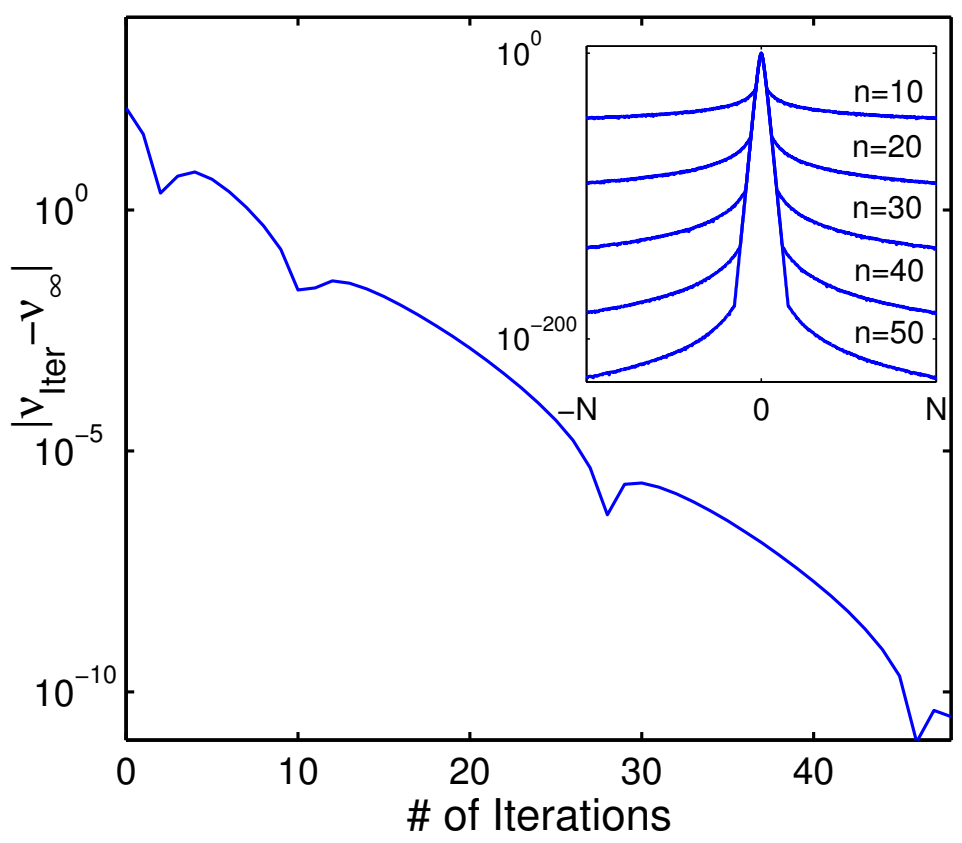

Figure 3.12: Convergence of the stationary rate as a function of the number of iterations. Inset: Stationary density at various iterations. Already after a few iterations, the iterated rate and density converge with a high accuracy. Each iterations takes only a fraction of a second on a standard desktop computer $\left(N=10^{4}\right)$. 


\subsection{Linear population response for arbitrary input frequencies}

In the previous section, I used the matrix representation of the Fokker-Planck operator $\hat{L}$ to compute the stationary response properties of the generalized $\theta$-neuron. In this section, the advantage of the approach will become apparent: Using the spectrum and eigenfunctions I will determine the response to small time-varying stimuli using the method described in Sec. 3.10 with very high accuracy.

First, the case $\alpha=0$, i.e. the classical $\theta$-neuron is considered in the (i) subthreshold, noise driven regime, (ii) the critical regime with $I_{0}=0$ and (iii) the suprathreshold regime, where $\sigma=0$.

\subsubsection{Dynamic responses for $\alpha=0$ to a current and noise modulation}

\subsubsection{Subthreshold regime $I_{0}<0$}

In Fig. 3.13, the linear response amplitude and phase for both types of modulation for different values of the stationary rate $v_{0}$ is shown. The linear response amplitude exhibits a resonance maximum at approximately $v_{0}$ and then decays rapidly to zero. The corresponding phase lag drops to $-\pi$ and shows a small dip at a multiple of the resonance frequency. Obviously, there is no frequency threshold, above which a sinusoidal stimulus cannot be transmitted through the population. However, frequencies above the maximum in the transmission curve are strongly damped. Thus, as cut-off frequency, the frequency is chosen at which the response amplitude maximizes. To determine this frequency we look again at the decomposition of $\hat{L}_{1}(\theta, z) P_{0}(\theta, z)$ in eigenfunctions of $\hat{L}_{1}(\theta, z)$ :

$$
\hat{L}_{1}(\theta, z) P_{0}(\theta, z)=\sum_{k} c_{k} P_{\lambda_{k}}(\theta, z)
$$

Since the mean value of the left hand side vanishes, the first coefficient $c_{1}$ is zero and because $\hat{L}_{1}(\theta, z)$ incorporates one derivative $\partial_{\theta}$, the sum has its maximum contribution for $k=2$. For $k>2$ the coefficients $c_{k}$ rapidly decay to zero. That means that due to the expansion in Eq. (3.54) the second eigenvalue also determines the cut-off frequency:

$$
f_{\text {cutoff }}=\frac{\operatorname{Im} \lambda_{2}}{2 \pi}
$$

\subsubsection{Critical regime $I_{0}=0$}

The response amplitude and phase are shown in Fig. 3.14. In this regime, where the $\theta$-neuron reflects best the dynamics of a saddle-node bifurcation, the response is similar to the response in the subcritical regime: For both types of modulations, the response function exhibits a resonance which is now however more pronounced. The resonance is again more pronounced for modulations in the noise amplitude than for modulations in mean input.

\subsubsection{Suprathreshold regime $I_{0}>0$}

In Fig. 3.15, the response curves in the suprathreshold noise-free regime are displayed. As expected, the response amplitude exhibits a pronounced peak at the stationary firing frequency and, at the same frequency, the phase shows a step-like change from 0 to $-\pi$. 

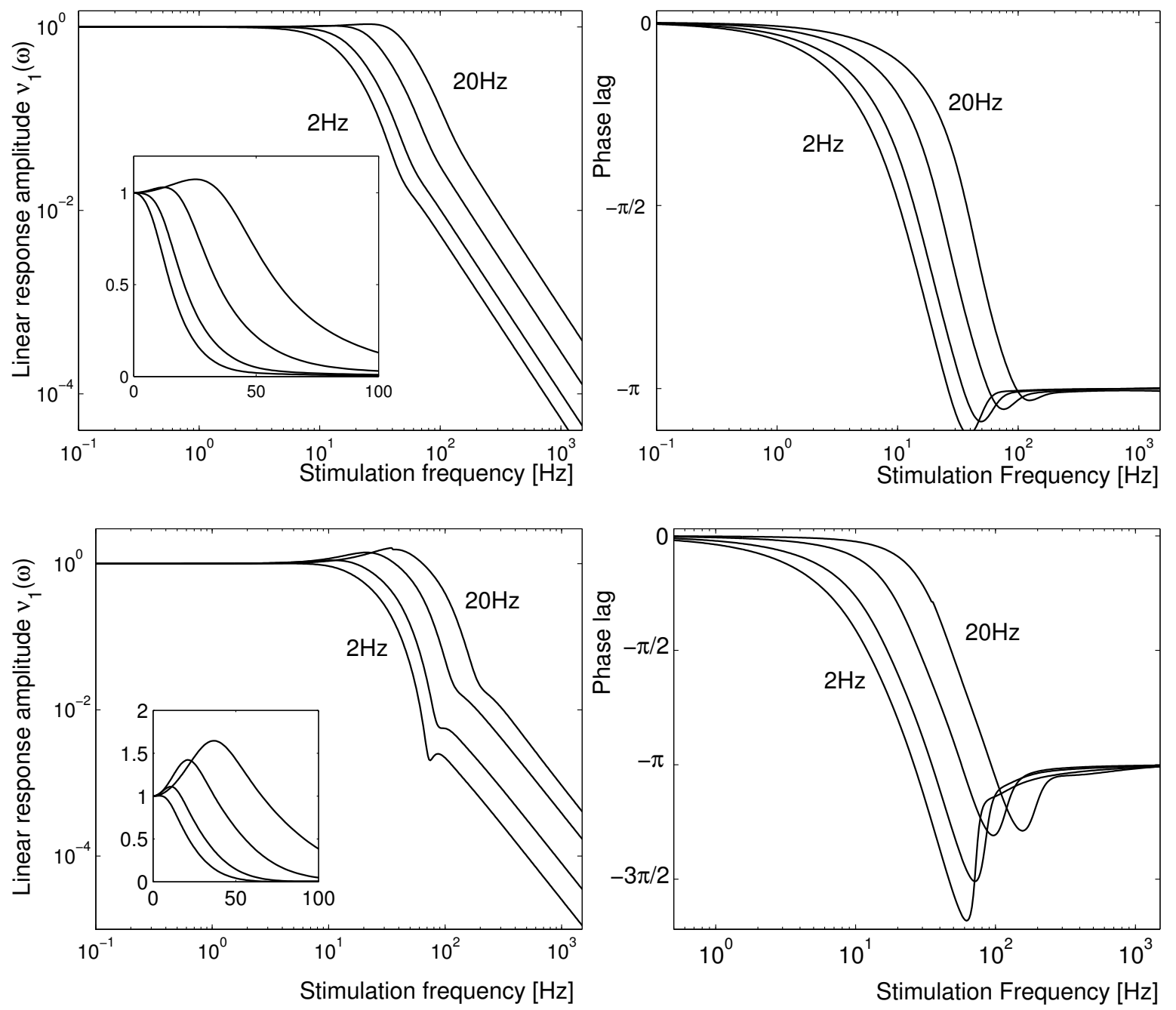

Figure 3.13: Subthreshold response amplitude (left) and phase (right) for different values of $v_{0}$ $(2,5,10,20 \mathrm{~Hz}), \tau_{c}=10 \mathrm{~ms}, I_{0}=5 \cdot 10^{-2}$ and $\alpha=0$ in the case of a modulation of the mean input current (top) and the noise amplitude (bottom). In the case of a current modulation, the amplitude exhibits a maximum at approximately $v_{0}$ and decays rapidly $\propto \omega^{-2}$ for larger values of $\omega$. The relative phase lag drops from zero to $-\pi$ and shows a small dip at the resonance frequency. The insets show the response amplitude for the different rates on a non-logarithmic scale. The resonance for modulations in the noise amplitude is more pronounced than for modulations in the mean input. 

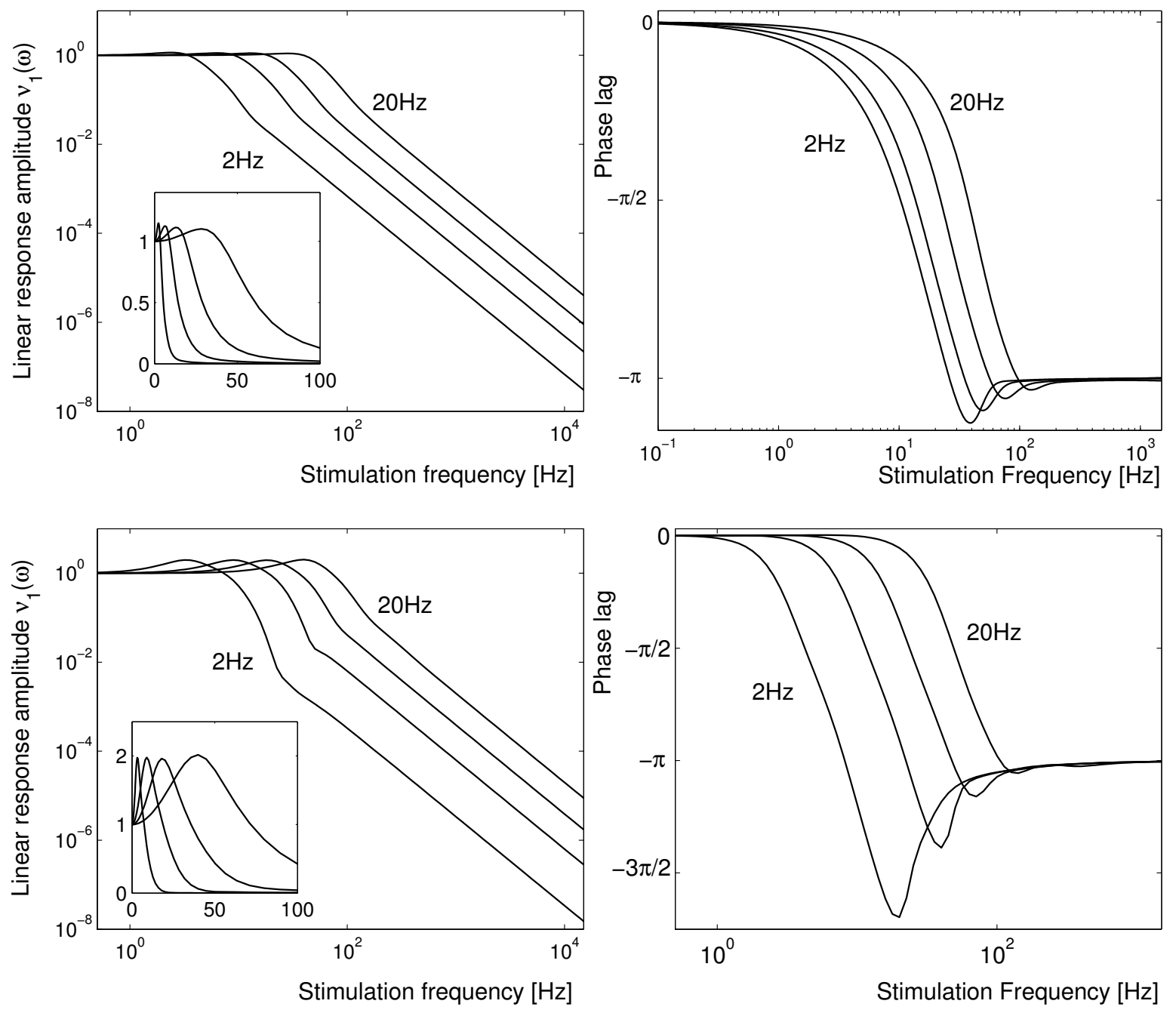

Figure 3.14: Critical response amplitude (left) and phase (right) for different values of $v_{0}$ $(2,5,10,20 \mathrm{~Hz}), \tau_{c}=10 \mathrm{~ms}, I_{0}=0$ and $\alpha=0$ in the case of a modulation of the mean input current (top) and the noise amplitude (bottom). For the current modulation, the amplitude exhibits a maximum at approximately $v_{0}$ and decays rapidly $\propto \omega^{-2}$ for larger values of $\omega$. The relative phase lag drops from zero to $-\pi$ and shows a small dip at the resonance frequency. The insets show the response amplitudes for the different rates on a non-logarithmic scale. For modulations in the noise amplitude, the resonances are more pronounced. 

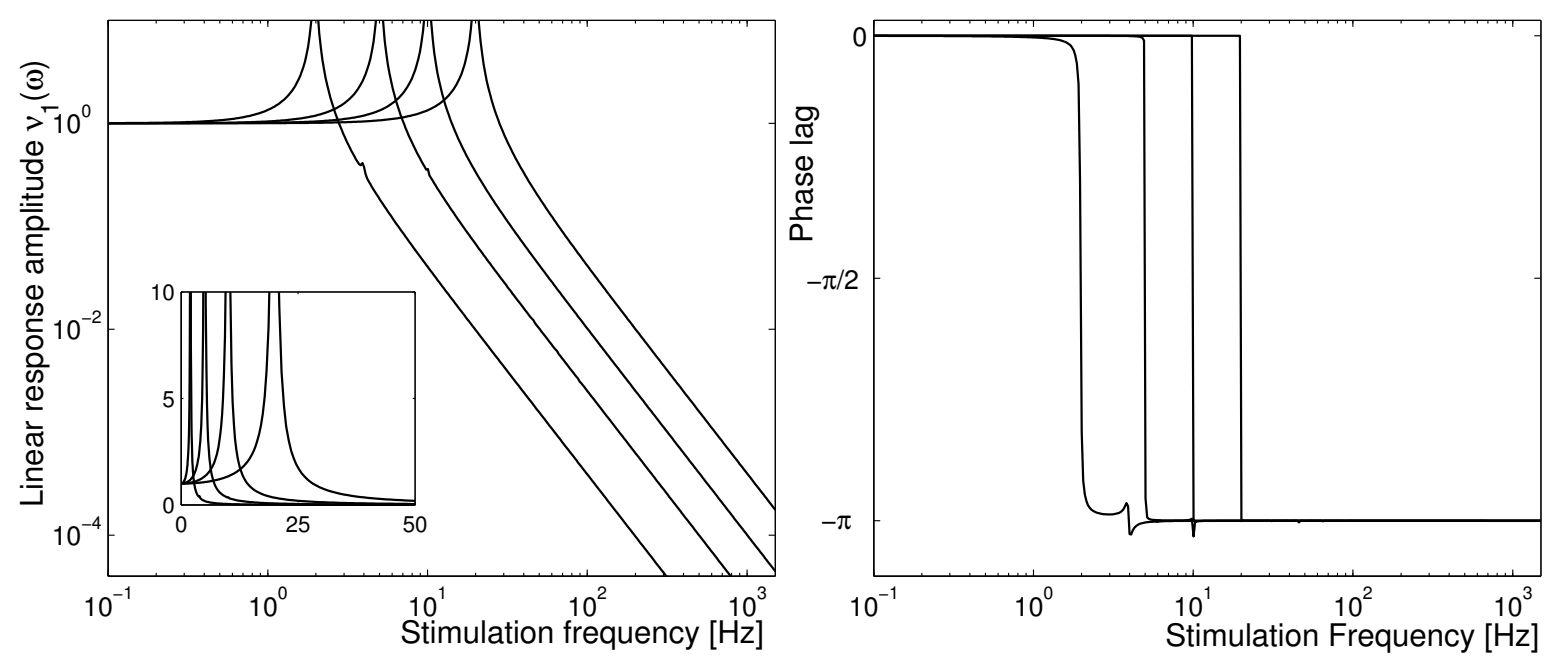

Figure 3.15: Suprathreshold response amplitude (left) and phase (right) for different values of $v_{0}$ $(2,5,10,20 \mathrm{~Hz}), \sigma=0$ and $\alpha=0$ in the case of a modulation of the mean input current. The amplitude exhibits a sharp resonance at $v_{0}$ and decays rapidly $\propto \omega^{-2}$ for larger values of $\omega$. The relative phase lag drops step-like from zero to $-\pi$. The inset shows the response amplitude for the different rates on a non-logarithmic scale.

In the limit $\sigma=0$, the eigenvalues, eigenfunctions and the full transmission function can be computed analytically. Direct integration of the eigenvalue Eq. (3.59) gives:

$$
P_{k}(\theta)=-\frac{\sqrt{8}}{\sqrt{I_{0}^{-3 / 2}\left(I_{0}+1\right)}} \frac{\exp (-2 i k \arctan (\tan (\theta / 2) / \sqrt{J}))}{I_{0}(1+\cos \theta)+(1-\cos \theta)}, \quad \lambda_{k}=2 i \sqrt{I_{0}} k, \quad k \in \mathbb{Z}_{0}
$$

The coefficients $c_{k}$ from Eq. (3.54) are given by:

$$
c_{ \pm 1}=\mp \frac{i}{2 \sqrt{I_{0}} \tau} ; \quad c_{k}=0 \quad k \neq \pm 1
$$

The modulated density and rate follow from straightforward substitution:

$$
\begin{aligned}
\tilde{P}(\theta, t) & =\left(\left(\frac{c_{1}}{i \omega-\lambda_{1}}\right) P_{1}(\theta)+c . c\right) e^{i \omega t} . \\
\Rightarrow\left|v_{1}(\omega)\right| & =\frac{1}{1-\left(\omega \tau /\left(2 \sqrt{I_{0}}\right)\right)^{2}}
\end{aligned}
$$

In contrast to the LIF and conductance-based models, the classical $\theta$-neuron exhibits only a single peak at its stationary rate, which is due to the sign-reversal symmetry $\theta \rightarrow-\theta$ in Eq. (3.10). With $\alpha>0$ this symmetry is lifted and the response amplitude exhibits resonances also at multiples of the stationary rate.

Taken together, these results demonstrate that our method is applicable in three regimes of neuronal dynamics for realistic values of the stationary rate and MP correlation. Before I show the impact of an increased AP onset speed on the response, I will explore the transition in the asymptotic regimes for large frequencies. While this is more of theoretical interest, it demonstrates the superiority of the 

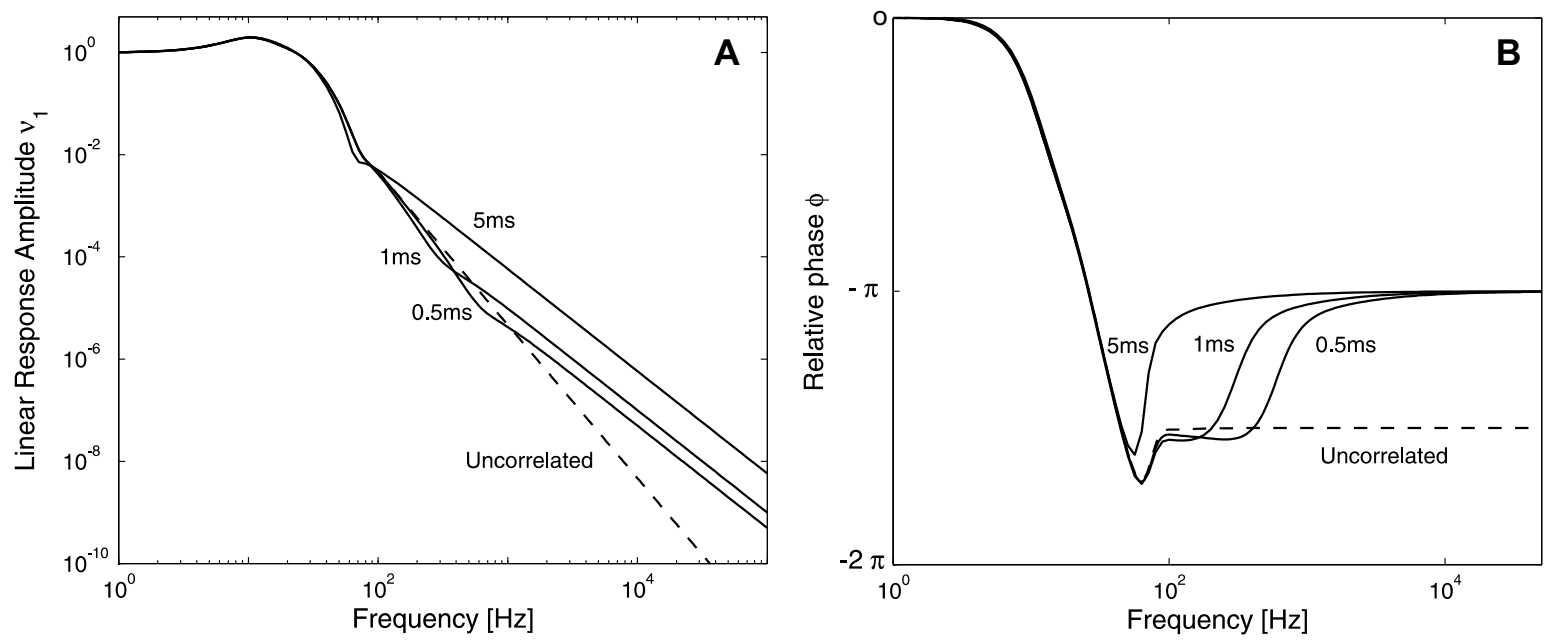

Figure 3.16: (A) Cross-over from a $\omega^{-3}$ decay to a $\omega^{-2}$ decay of the response amplitude for different values of the correlation time $\tau_{c}$ in the case of a modulation in the noise amplitude. (B) Relative phase of the response for the same values of $\tau_{c}$. The phase decays first to $-3 \pi / 2$ and then goes over to $-\pi$. The input current and the noise amplitude is in all three cases fixed. $\left(I_{0}=0, \sigma=10^{-3}\right)$, leading to a stationary rate of $\approx 7 \mathrm{~Hz}$. For an input correlation time of $5 \mathrm{~ms}$, the cross-over is no longer visible.

matrix method, which allows to study the asymptotic decay of the transmission function even at very large frequencies.

\subsubsection{Transitions of the asymptotic decay for large frequencies}

As pointed out in Sec. 3.8, the linear response amplitude for the case of a noise modulation decays as $\omega^{-2}$ for a colored input current, while it decays as $\omega^{-3}$ in the limit $\tau_{c} \rightarrow 0$, i.e. for white noise input. For small values of $\tau_{c}$, a cross-over is expected between these two limiting behaviors. This can indeed be observed as depicted in Fig. 3.16. The linear response amplitude decays as $\omega^{-3}$ up to a cross-over frequency which grows with decreasing values of $\tau_{c}$, whereupon it decays as $\omega^{-2}$. The relative phase of the response reflects this by exhibiting a plateau at $-3 \pi / 2$ and then going to $-\pi$. The cross-over is, however, only visible for small correlation times.

In Fig. 3.17, the response amplitude and phase are shown for different values of the parameter $\delta$. The decay is $\propto \omega^{-2}$ up to a cross-over at a frequency $f_{\text {break. }}$. For larger frequencies the decay is $\propto \omega^{-1}$ and the relative phase goes to $-\pi / 2$. This cross-over can however be at very large frequencies for small values of $\delta$. The same argument holds for the generalized model, since only the spike onset is modeled differently.

\subsubsection{Dynamic responses for $\alpha>0$}

\subsubsection{Excitable regime}

In this section, I discuss the response properties for the case $\alpha>0$ for the two scenarios of a modulation of the noise amplitude and for a modulation of the mean current. It turns out that for both cases, 

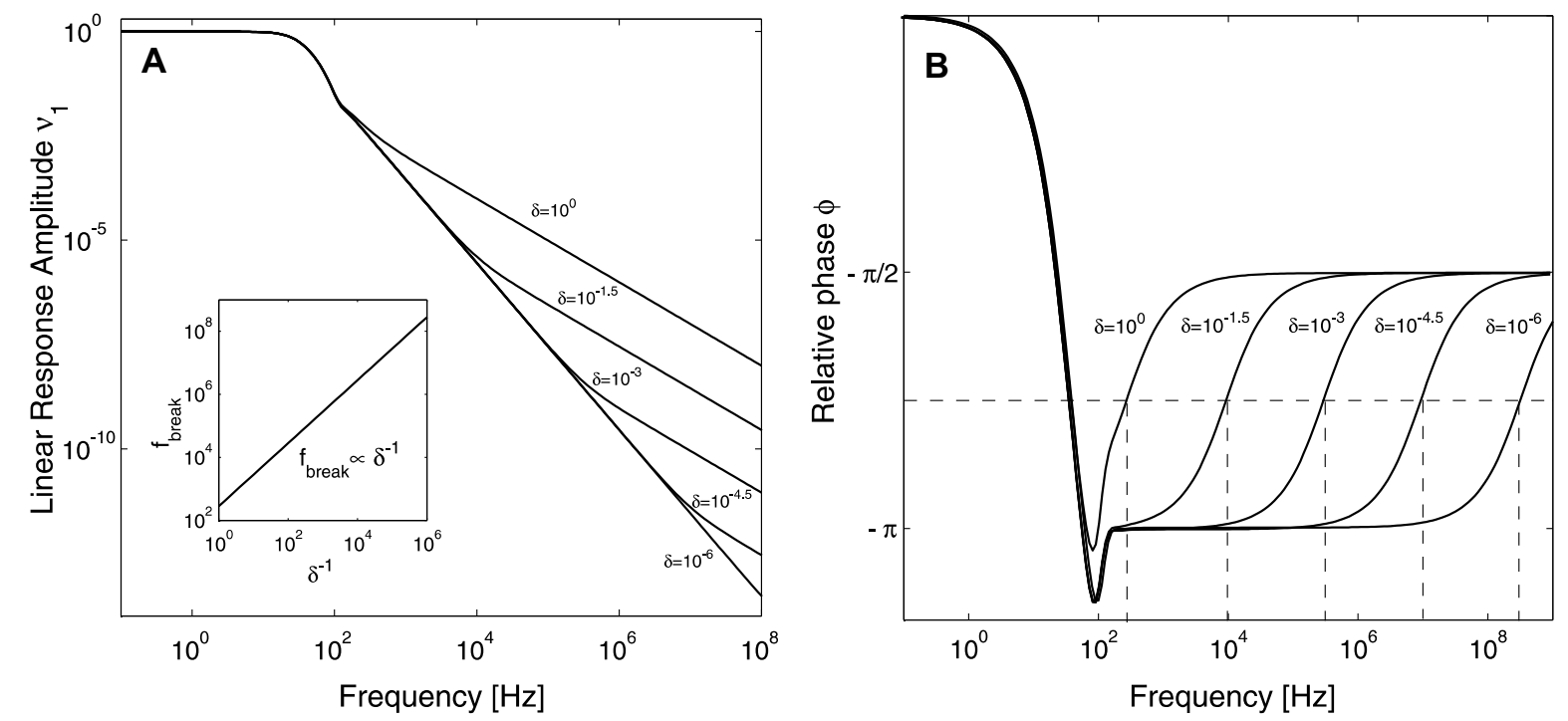

Figure 3.17: (A) Cross-over from a $\omega^{-2}$ decay to a $\omega^{-1}$ decay of the response amplitude for different values of $\delta$. The inset shows the frequency $f_{\text {break }}$ at which the cross-over takes place. It exhibits a linear relation over several orders of magnitude with a slope of 1, i.e $f \propto \delta^{-1}$. (B) Relative phase of the response for different values $\delta$. The phase decays first to $-\pi$ and then goes over to $-\pi / 2$. The cross-over is defined at the points where the phase crosses the line $-3 \pi / 4$ from below.

the frequency up to which input stimuli can be transmitted can be much larger than in the case $\alpha=0$.

In Fig. 3.18, the response behavior for increasing values of $\alpha$ is displayed. For both current and noise modulation, the response curve decays much slower for increasing values of $\alpha$, than for the standard $\theta$-neuron. This is surprising, because the eigenvalue spectrum hardly changes upon an increase in $\alpha$, as shown before in Fig. 3.10. It turns out, however that eigenfunctions belonging to eigenvalues with very large imaginary parts start to contribute in the expansion of $\hat{L}_{1} P_{0}$ into eigenfunctions.

To assess the impact of increasing values of $\alpha$ on the transmission function systematically, the response amplitude at $500 \mathrm{~Hz}$ and $1 \mathrm{kHz}$ as a function of $\alpha$ is shown in Fig. 3.19. The response for modulations of the noise amplitude is typically larger than the response for a mean current modulation, whereby both are growing with increasing values of $\alpha$. The fluctuations in the curve are due to resonances from higher eigenvalues. One should keep in mind that the stationary rate of the neuron is fixed at $20 \mathrm{~Hz}$, which is almost two orders of magnitude smaller than the frequency at which the response amplitude is measured.

\subsubsection{Suprathreshold regime}

In Fig. 3.20, the response amplitude and phase in the suprathreshold regime is displayed. In contrast to the classical $\theta$-neuron, the response of the generalized model also exhibits resonances at multiples of its stationary firing rate, which are more and more damped with increasing values of $\omega$. 

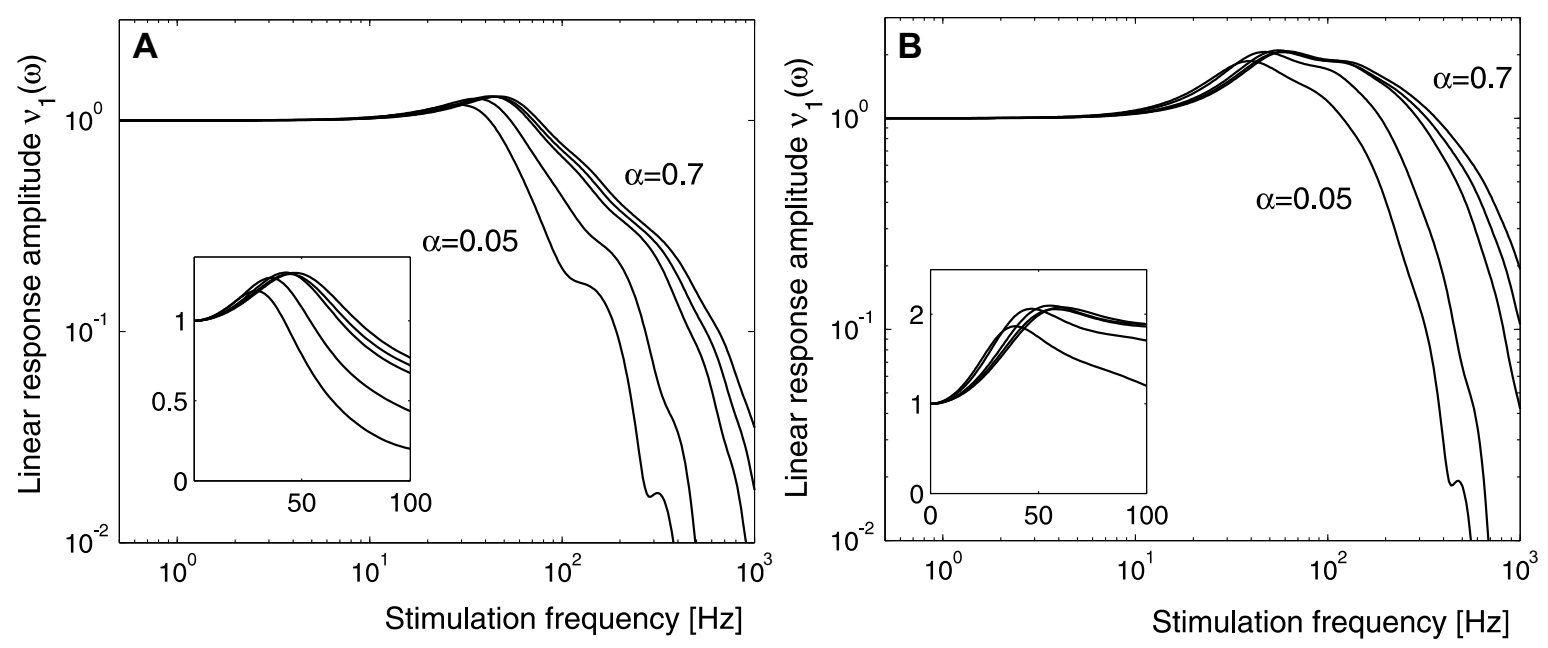

Figure 3.18: (A) Response amplitude for different values of $\alpha(0.05,0.1,0.5,0.6,0.7), \tau_{c}=10 \mathrm{~ms}, I_{0}=$ $10^{-1}$ and $v_{0}=20 \mathrm{~Hz}$ in the case of a modulation of the mean input current and (B) noise amplitude. For increasing values of $\alpha$ the response amplitude increases. For $\alpha=0.7$, frequencies up to $1 \mathrm{kHz}$ can be transmitted practically unattenuated.

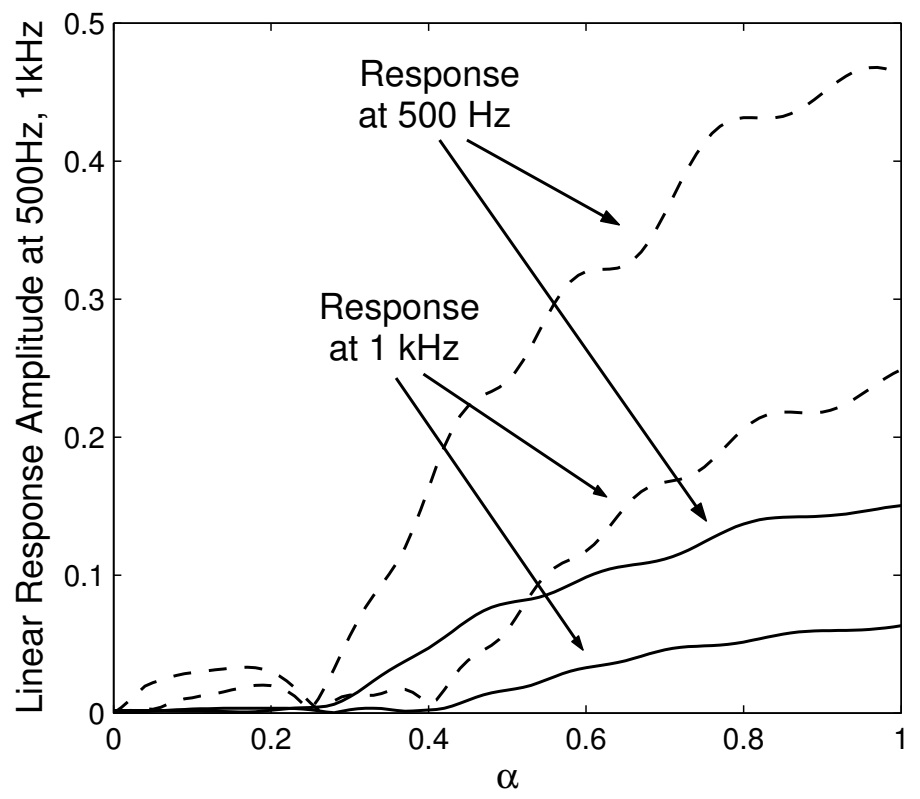

Figure 3.19: Response amplitude at $500 \mathrm{~Hz}$ and $1 \mathrm{kHz}$ as a function of the parameter $\alpha$. Solid: Response amplitude to modulations in the mean current. Dashed: Response to modulations in the noise amplitude. At both frequencies, the noise response is typically larger the mean current response. Both are growing with increasing values of $\alpha$, showing a number of resonances. Further parameters are: $I_{0}=-0.1$. The noise strength $\sigma$ was adjusted for each value of $\alpha$ to give a stationary rate of $20 \mathrm{~Hz}$. 

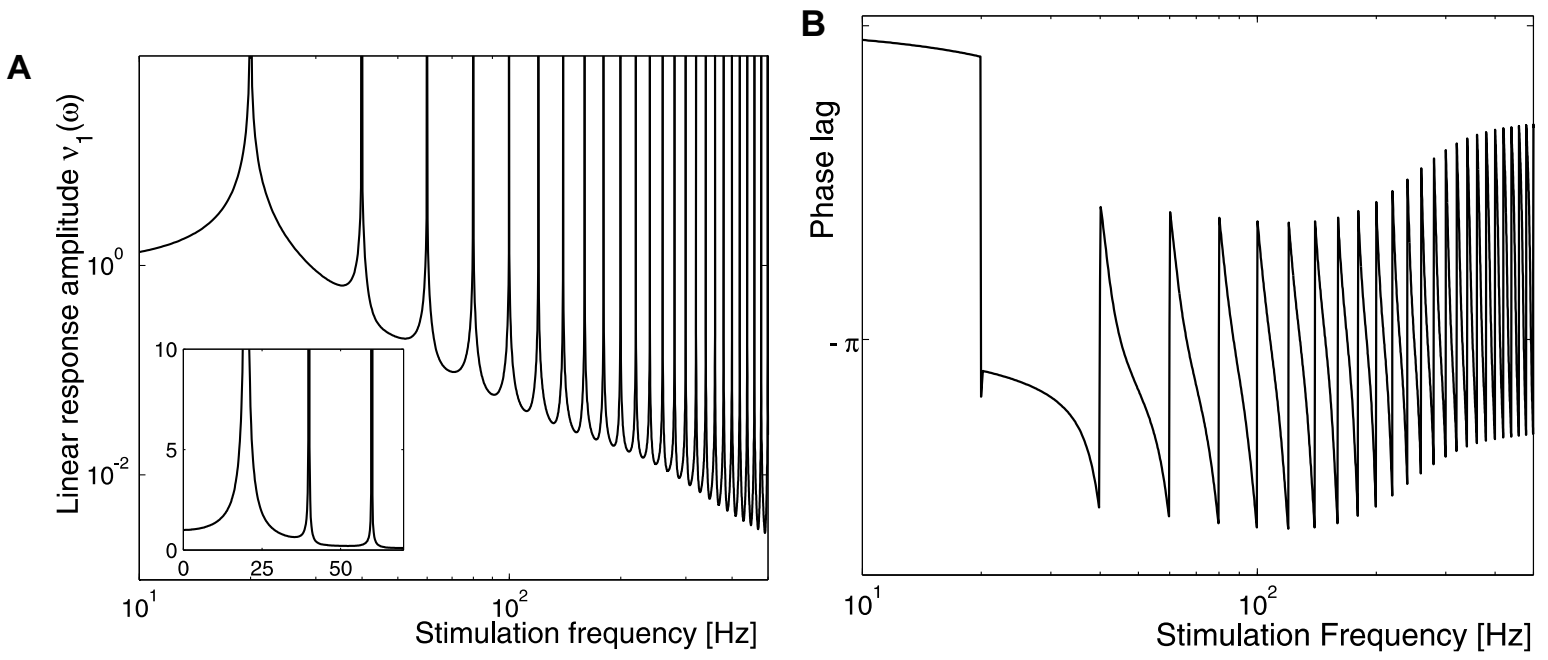

Figure 3.20: (A) Suprathreshold response amplitude and (B) phase for $v_{0}=20 \mathrm{~Hz}, \sigma=0$ and $\alpha=0.2$ in the case of a modulation of the mean input current. In contrast to the classical $\theta$-neuron, the amplitude exhibits resonances not only at $v_{0}$, but also at integer multiples of the stationary rate. For larger frequencies it decays rapidly $\propto \omega^{-2}$ for larger values of $\omega$. The large number of resonances are reflected in the relative phase of the response. The inset show the response amplitude on a non-logarithmic scale.
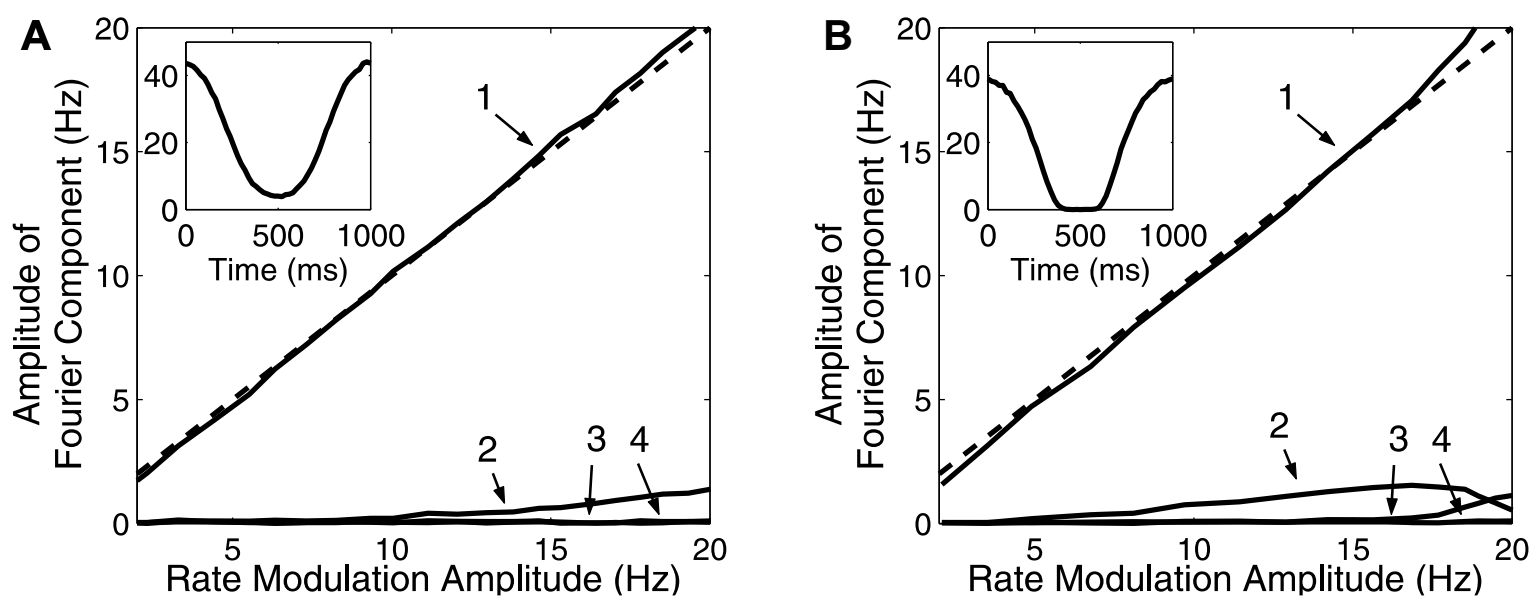

Figure 3.21: Amplitude of the first four Fourier components as a function of overall modulation amplitude of the population averaged firing rate for $(\mathbf{A})$ modulations in the mean input current and (B) for modulations in the noise amplitude $(\alpha=0.7)$. The mean output rate is $20 \mathrm{~Hz}$, the modulation frequency $1 \mathrm{~Hz}$. The dashed line is the diagonal. Up to amplitudes close to the mean output rate, the first Fourier component is indistinguishable from the diagonal, indicating that the response is essentially linear. Starting from amplitudes comparable to the mean rate, the influence of higher order Fourier components becomes substantial. The insets show the rate modulation for a modulation amplitude of $20 \mathrm{~Hz}$. 


\subsubsection{Nonlinear response for large stimulation amplitudes}

So far, only the linear response transmission functions were considered. The obtained results are thus, strictly speaking, only valid in the limit in which the stimulation amplitude goes to zero. Here, I show, however, that the linear response covers a large range of input amplitudes. In principle, the same matrix method employed for the linear response theory could be used if higher order Floquet modes were taken into account (Reichl, 1988). Here, I explore this regime, however, by direct numerical simulation of Eq. (3.12). In Fig. 3.21, the amplitude of the first four Fourier modes of the rate response as a function of the overall amplitude of the rate modulation are shown. For both types of modulations, the first Fourier component clearly dominates the response up to amplitudes close to the mean rate, where nonlinearities are naturally expected, as there are no negative firing rates. This shows that the linear response theory, although rigorously valid for small modulation amplitudes only, predicts the response in a large dynamical range of input amplitudes.

\subsection{Summary and discussion}

The stationary and dynamic response properties of the generalized $\theta$-neuron, a canonical model of Type-I excitable membranes, with adjustable AP onset speed were assessed in the presence of a fluctuating correlated background noise. The stationary response properties were calculated analytically in the limit of fast-decaying synaptic currents. To calculate the dynamic response properties, I introduced a new approach which is based on the expansion of the corresponding Fokker-Planck operator into a complete set of orthonormal functions, leading to a sparse matrix representation. I then computed the eigenvalues and eigenfunctions of this matrix using an iterative scheme, the Arnoldi method. The high frequency limit was calculated analytically. It turned out, that the response amplitude decays as $\omega^{-\gamma}$, where $\gamma$ depends on the kind of stimulation and, surprisingly, the phase at which a spike is emitted. As soon as this point differs from $\pi$, where the dynamics is insensitive to external inputs, the exponent $\gamma$ is 1, giving the same asymptotic response behavior as conductance-based neuron models. Using the eigenvalues and eigenfunctions, I then presented a method to evaluate the dynamic response in linear response theory. There, I found that for the classical $\theta$-neuron model the response exhibits a cut-off behavior: For a modulation in the mean input current as well as for a modulation in the noise amplitude, frequencies above the stationary rate of the neuron are strongly damped. In the generalized $\theta$-neuron, the damping in the regime up to $1 \mathrm{kHz}$ is substantially reduced for both types of input modulations when the AP onset speed is increased, although the high frequency limit is the same as for the classical $\theta$-neuron. The response amplitude for the noise amplitude modulation is typically much larger than the response amplitude for the mean input modulation. The linear response theory, although only derived for small modulations of the input current, turned out to be valid in a large dynamical range, which I demonstrated by direct numerical simulations. Amplitudes of the rate modulation up to the mean output rate turned out to be well described by the linear theory.

Simple phenomenological, yet dynamically realistic models of cortical neurons are of key importance for studies in theoretical neuroscience, starting from studies on spike timing to large scale network simulations or analytical network studies. While stationary response properties, such as mean firing rates or processes which operate on long time scales, e.g. adaptation, were assessed in many models, studies on the dynamic response properties are rare. Most of these studies considered the dynamic response properties of the class of IF models (Knight, 1972; Brunel et al., 2001; Lindner \& SchimanskyGeier, 2001; Fourcaud \& Brunel, 2002). In these studies, it was demonstrated that IF models can 
relay incoming stimuli instantaneously. Recently it was shown, however, that this response behavior strongly disagrees with the response of conductance-based models and rather represents an oversimplification of the model than a feature of real neurons (Naundorf et al., 2003; Fourcaud-Trocmé et al., 2003). While in (Naundorf et al., 2003, 2005b), the response properties of the classical $\theta$-neuron were investigated, Fourcaud-Trocmé et al. (2003) studied another phenomenological neuron model, the EIF model, which mimics the dynamic response properties of a conductance-based model. The results presented in this chapter corroborate and extend some of their results using a generalized model of the classical $\theta$-neuron (Ermentrout \& Kopell, 1986; Gutkin \& Ermentrout, 1998) which, in contrast to IF models, incorporates a dynamic AP onset. While the classical $\theta$-neuron model was originally studied in the suprathreshold, noise-free case (Ermentrout \& Rinzel, 1984; Ermentrout \& Kopell, 1986), recent studies focused on the response in the presence of fluctuating input currents (Gutkin \& Ermentrout, 1998; Lindner et al., 2003; Brunel \& Latham, 2003). These studies indicated that in a large parameter regime the $\theta$-neuron exhibits stationary response properties similar to cortical neurons, e.g. a realistic shape of the f-I curve and irregular firing in the subthreshold regime.

Despite these results, a major point of criticism, questioning the biological relevance of the model remained: While the $\theta$-neuron reflects the dynamics at the onset to repetitive firing, it lacks the sharp AP upstroke found in more detailed conductance-based models and real neurons (FourcaudTrocmé et al., 2003). It was further argued that this deficiency results in a high frequency limit of the linear response amplitude, which decays too fast $\propto \omega^{-2}$, while the linear response amplitude of conductance-based neuron models only decays $\propto \omega^{-1}$. To address these issues, I generalized the classical $\theta$-neuron, incorporating an adjustable AP onset speed, thereby mimicking the fast sodium activation at the AP onset. Surprisingly, the results presented in this chapter demonstrate that the high frequency limit, does not depend at all on the speed at the AP onset, but rather on the phase variable at which APs are emitted. If at this point the dynamics is insensitive to external inputs, as in the classical $\theta$-neuron, the decay of the linear response amplitude is at least $\propto \omega^{-2}$, whereas the decay is always $\propto \omega^{-1}$ if the dynamics is not completely insensitive to external inputs, as is the case in conductance-based neuron models and real neurons. Moreover, the full transmission function reveals that the onset of the high-frequency limit can be shifted to very high frequencies if the speed of the AP onset is increased. These results question the relevance of the high frequency limit as a criterion for the typical transmission speed of neuron models.

In Fig 3.22, the response amplitude curves for the $\theta$-neuron model and the generalized $\theta$-neuron model are summarized and compared with direct numerical simulations of the response of the LIF model. The $\theta$-neuron exhibits a cut-off behavior in its response amplitude to both types of input modulations. Frequencies up to the stationary firing rate can be transmitted unattenuated larger frequencies are strongly damped. For an increasing onset speed and fixed stationary rate the resonance maximum shifts only slightly to larger frequencies, a considerable change occurs, however, at intermediate frequencies up to $1 \mathrm{kHz}$. In this regime the response amplitude is substantially lifted to much larger transmission amplitudes. This effect is much more pronounced for the case of a modulation in the noise amplitude than for modulations in the mean input current, leading to an undamped response for frequencies up to $200 \mathrm{~Hz}$. The LIF model, on the other hand, shows a completely artificial response behavior. The transmission function, for both types of modulations does not decay at all, even for frequencies up to $1 \mathrm{kHz}$. For modulations in the noise amplitude, the transmission function can even grow for increasing stimulation frequencies.

For the computation of the linear response amplitude I did not resort to direct numerical simulations, but used a method based on the eigenfunctions and eigenvalues of the Fokker-Planck operator, describing the dynamics of the probability density function in the presence of a temporally correlated fluctu- 


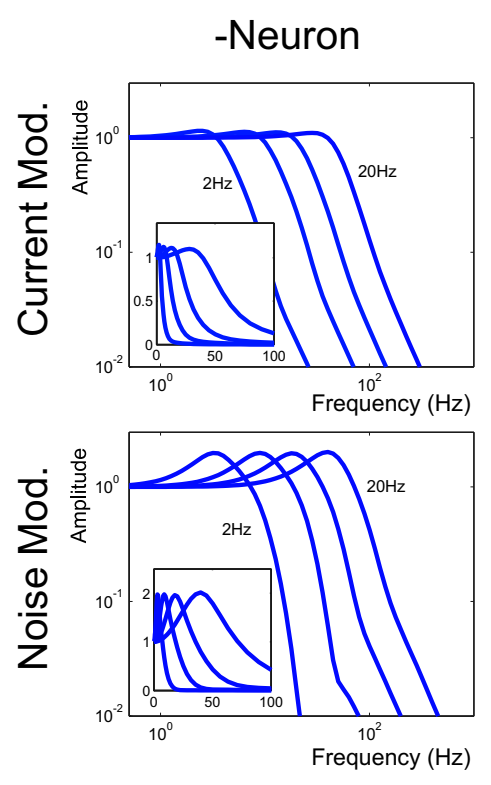

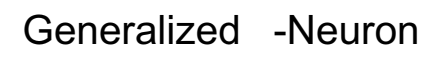
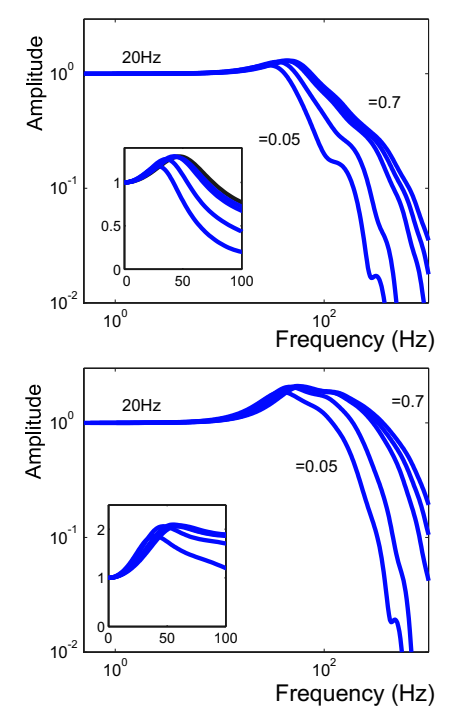

\section{LIF model}
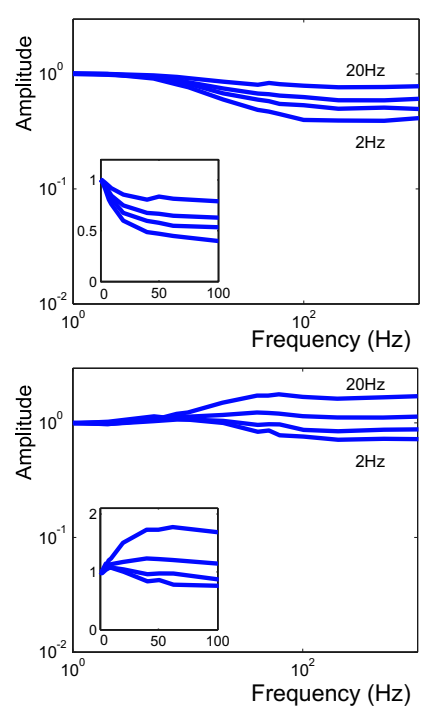

Increasing Action Potential Onset Speed

Figure 3.22: Response amplitude for increasing values of the AP onset speed. In the left column the response of the $\theta$-neuron for modulations in the mean input current and the noise amplitude is shown for different values of the stationary firing rate. The response exhibits a cut-off behavior, frequencies larger than the stationary firing rate are strongly damped. The middle column shows the response of the generalized model for both types of modulation and a stationary rate of 20 $\mathrm{Hz}\left(\tau_{c}=10 \mathrm{~ms}\right)$. For increasing values of the AP onset speed the response amplitude grows for frequencies in the interval from $100 \mathrm{~Hz}$ to $1 \mathrm{kHz}$, while the resonance maximum only slightly shifts to larger frequencies. The response of the noise modulation is much larger in this interval than the response to modulations in the mean current. For comparison, the right column shows the response of the leaky integrate-and-fire (LIF) model. The response amplitude does not decay for large frequencies, for modulations of the noise amplitude it can even grow with increasing input frequencies. Parameters in the LIF simulation are as in (Brunel et al., 2001), except $\tau_{s}=10 \mathrm{~ms}$, $\sigma=5 \mathrm{mV}, I_{0}=14.6 ; 16.2 ; 17.5 ; 19.5 \mathrm{mV}$ for a mean firing rate of $2,5,10,20 \mathrm{~Hz}$ (From Naundorf et al. (2005a)). 
ating input current. While this approach is in general well-known (see e.g. (Risken, 1996) and (Knight et al., 2000; Mattia \& Del Giudice, 2002) for an application to the non-leaky IF model in the presence of an uncorrelated background noise), I derived a sparse matrix representation, for which I computed eigenvalues and eigenfunctions with very high numerical accuracy using a fast iterative scheme, the Arnoldi method (Trefethen \& Bau, 1997; Lehoucq et al., 1998). Compared to previous studies on dynamic response properties (Brunel et al., 2001; Fourcaud \& Brunel, 2002; Fourcaud-Trocmé et al., 2003), this allowed for the computation of the linear response properties with an accuracy that would be hard to meet by a direct simulation of the single neuron dynamics.

Besides this, our results provide a direct link to experiments. In a recent study (Brumberg, 2002) it was shown that the AP width in neocortical neurons is strongly correlated with the critical frequency up to which a neuron can phase lock to sinusoidal input stimulations. This is indeed the same result which I found for the generalized $\theta$-neuron: With an increasing AP onset speed, the response amplitude shifts to larger frequencies, enabling the model to respond to frequencies much larger than its stationary rate. In a second study, which was motivated by the fast response properties of LIF models (Lindner \& Schimansky-Geier, 2001; Silberberg et al., 2004), it was demonstrated that cortical neurons subject to fluctuating input currents change their instantaneous firing much faster when stimulated with a step input in the noise amplitude than with a step mean input current (Silberberg et al., 2004). This behavior is well reproduced by the generalized $\theta$-neuron. For increasing values of the AP onset speed, the response amplitude at high frequencies is much larger for stimulations of the noise amplitude, compared to stimulations of the mean input current. Both results strongly suggest that the generalized $\theta$-neuron, despite its simplicity and analytical tractability, captures well a key aspect the AP generating mechanism of real neurons. Future experimental studies will have to show to what extent the generalized $\theta$-neuron predicts correctly the dependence of the dynamic response properties on the AP generating mechanism.

\subsection{Perspectives}

Ultimately, this study aims at understanding the dynamics of cortical neurons and their computational properties. I've started this chapter by discussing experiments which assessed the dynamic response properties of single neurons. Although these studies are a first step in the experimental assessment of the transformation properties of real neurons in an in vivo like regime, a systematic investigations of these properties is still to be done. In particular, the connection between AP onset dynamics and population coding in the presence of fluctuating background currents can be tested in in vitro recordings of cortical neurons. A first important step in this direction is to investigate in detail the AP initiation dynamics in cortical neurons, which I will do in the following section.

A very important issue, which I didn't cover in this study, is the role of feedback network connections. In theoretical studies, using a simple binary neuron model, it was shown that strong inhibitory feedback connections can lead to a substantial increase in the temporal precision, with which a population of such neurons can follow time-dependent stimuli (van Vreeswijk \& Sompolinsky, 1996, 1998). Indeed, a straightforward generalization of the results in these studies shows that this increase in temporal precision is not restricted to the neuron model used in these studies, but also holds for the generalized $\theta$-neuron model. Feedback connections might therefore establish a second independent mechanism which enables cortical neuronal populations to exhibit a rapid response to transient stimuli. 


\title{
4 Anomalous action potential initiation in cortical neurons
}

\begin{abstract}
The coding properties of the generalized $\theta$-neuron, which I derived in the previous chapter, indicate that the speed of neuronal population coding is closely linked to the AP initiation dynamics. For a smooth AP initiation dynamics, a population can only faithfully transmit slowly-modulated input stimuli. Conversely, with a more rapid AP initiation dynamics, the population encoding turned out to be very precise, independent of the neuron's membrane time constant or its stationary firing rate. From the elucidation of the AP onset dynamics in real neurons, one would thus expect to learn about the coding properties of neuronal populations in the cortex. Moreover, the quantitative analysis of the dynamics of AP initiation in cortical neurons allows to calibrate neuron models, such as the generalized $\theta$-neuron model.
\end{abstract}

In this chapter, I thus assess the AP onset dynamics in in vitro and in vivo recordings of cortical neurons, and compare the dynamics with recently proposed Hodgkin-Huxley type models of cortical neurons. Surprisingly, it turns out that two key features of cortical AP initiation are not reflected by these models: A fast initiation dynamics of APs and a large variability of AP onset potentials. A detailed investigation further reveals that these two dynamical features are mutually exclusive in the framework of the Hodgkin-Huxley theory and cannot be achieved together, suggesting that a basic assumption of the theory is violated qualitatively (Naundorf et al., 2005c).

I will then discuss three possible biophysical mechanisms to explain this "anomalous" AP initiation dynamics of cortical neurons: Ion-channel stochasticity, intrinsic coupling of voltage-gated sodium channels and cooperative gating between ion channels. While the first mechanism does most likely not explain the observed dynamics of AP initiation, the latter two are realistic candidates.

\subsection{Action potentials: Energy budget considerations of the cortex}

In the Hodgkin-Huxley theory, the dynamics of AP initiation emerges from the dynamical interplay between voltage-gated channels. An important complementary view on the dynamics of neurons, is to consider the function they are optimized for as a consequence of evolutionary pressure. By revealing the underlying optimization principles, it might as well be possible to elucidate the coding principles realized in the nervous system.

Several recent studies pointed out that a major limiting factor in neural processing is the available energy budget (Attwell \& Laughlin, 2001; Laughlin \& Sejnowski, 2003; Lennie, 2003). In these studies it was suggested that in the cortical grey matter, more than $75 \%$ of the total energy is used for communication, i.e. to drive signals along axons and synapses. By comparing in detail the energy consumption of different neural processes, including the energy uptake during the emission of an AP, 
with estimations of the overall power consumption of the brain, Laughlin \& Sejnowski calculated the average firing rate that is compatible with the energy uptake of the cortex. For the cerebral cortex, it turned out that the permissible traffic is about 5 APs per neuron per second in rat and even less than 1 AP per second in human. The latter estimate is based on the fact that the energy uptake of the human brain accounts for $20 \%$ of the total energy supply of the whole body. Thus, already an increase in the mean firing rate per neuron by a factor of two could hardly be supported by the human metabolism. One would therefore expect that the AP initiating mechanisms are specifically tailored to support this sparse coding.

An important aspect of the analysis in this chapter is to uncover the dynamical features of cortical AP initiation. In the following chapter I will then discuss their functional implications in a phenomenological neuron model for time-dependent population coding.

\subsection{Hodgkin-Huxley type models of cortical neurons}

Several neuron models were proposed to describe the dynamics of cortical neurons in vivo. HodgkinHuxley type neuron models, which incorporate the detailed kinetics of many different voltage-gated channels, are considered to be most realistic. In this chapter, I will compare the dynamics of AP initiation in cortical neurons with the dynamics of two recently proposed conductance-based neuron models. Both models were specifically constructed to match the subthreshold dynamics and the firing statistics of cortical neurons in response to fluctuating synaptic inputs.

\section{Model A}

Model A is a single compartmental Hodgkin-Huxley type neuron model (Destexhe et al., 2001). As shown by the authors of this study, the model reproduces the statistics of MP fluctuations and spiketrain statistics of neocortical neurons in vivo. In this model, the dynamics of the MP is given by:

$$
C_{m} \frac{d V}{d t}=-g_{L}\left(V-E_{L}\right)-I_{N a}(t)-I_{K d}(t)-I_{M}(t)-A^{-1} I_{s y n}(t),
$$

with the current densities:

$$
\begin{aligned}
I_{N a} & =\bar{g}_{N a} m^{3} h\left(V-E_{N a}\right) \\
I_{K d} & =\bar{g}_{K d} n^{4}\left(V-E_{K d}\right) \\
I_{M} & =\bar{g}_{M} p\left(V-E_{M}\right),
\end{aligned}
$$

where $C_{m}=1 \mu \mathrm{F} / \mathrm{cm}^{2}$ is the specific membrane capacitance, $g_{L}=0.045 \mathrm{mS} / \mathrm{cm}^{2}$ is the leak conductance density, and $E_{L}=-80 \mathrm{mV}$ is the leak reversal potential. $I_{N a}$ is the voltage-dependent $\mathrm{Na}^{+}$current and $I_{K d}$ is the 'delayed-rectifier' $\mathrm{K}^{+}$current underlying the generation of APs. $I_{M}$ is a non-inactivating $K^{+}$current causing spike frequency adaptation and $A$ is the total membrane area, which was assumed to $3.4636 \cdot 10^{4} \mu \mathrm{m}^{2}$. The peak conductances of the active currents are given by $\bar{g}_{N a}=51.6, \bar{g}_{K d}=10$ and $\bar{g}_{M}=0.5 \mathrm{mS} / \mathrm{cm}^{2}$. Their reversal potentials are $E_{N a}=50, E_{K d}=-90$ and $E_{M}=-90 \mathrm{mV}$. The functions $m(t), h(t), n(t), p(t)$ are dynamical activation variables, indicating the opening probability of channel subunits. They were described using the usual Hodgkin-Huxley type formalism (see Sec. 2.6) as first order differential equations with opening and closing rates $\alpha_{x}(V)$ and 
$\beta_{x}(V)$, respectively. The rates for the sodium channel activation are given as:

$$
\alpha_{m}=\frac{-0.32\left(V-V_{T}-13\right)}{\exp \left(-\left(V-V_{T}-13\right) / 4\right)-1}, \quad \beta_{m}=\frac{0.28\left(V-V_{T}-40\right)}{\exp \left(-\left(V-V_{T}-40\right) / 5\right)-1},
$$

and the rates for the inactivation variable $h(t)$ of the sodium channel as:

$$
\alpha_{h}=0.128 \exp \left(-\left(V-V_{T}-V_{S}-17\right) / 18\right), \quad \beta_{h}=\frac{4}{1+\exp \left(-\left(V-V_{T}-V_{S}-40\right) / 5\right)},
$$

where $V_{T}=-58 \mathrm{mV}$ and $V_{S}=-10 \mathrm{mV}$. The voltage-dependent rates of the $K^{+}$-current were defined as:

$$
\alpha_{n}=\frac{-0.032\left(V-V_{T}-15\right)}{\exp \left(-\left(V-V_{T}-15\right) / 5\right)-1}, \quad \beta_{n}=0.5 \exp \left(-\left(V-V_{T}-10\right) / 40\right)
$$

The rates of the activation variable $p$, describing the activation of non-inactivating $K^{+}$channels, were modeled as:

$$
\alpha_{p}=\frac{10^{-4}(V+30)}{1-\exp ((V+30) / 9)}, \quad \beta_{p}=\frac{-10^{-4}(V+30)}{1-\exp ((V+30) / 9)}
$$

The current $I_{M}$ is mainly responsible for spike-frequency adaptation, as described in detail in (Paré et al., 1998).

The total synaptic current consists of excitatory and inhibitory parts:

$$
I_{s y n}=g_{E}(t)\left(V-E_{E}\right)+g_{I}(t)\left(V-E_{I}\right),
$$

with the reversal potentials $E_{E}$ and $E_{I}$ of excitatory and inhibitory synaptic currents, respectively. The conductances $g_{E}(t)$ and $g_{I}(t)$ were modeled as Ornstein-Uhlenbeck processes with correlation times $\tau_{E}$ and $\tau_{I}$ (see Sec. 2.8.1).

To compare the AP dynamics between cortical neurons and the model in the presence of a fluctuating input, the model was used with the same parameter as in (Destexhe et al., 2001). To study the impact of the sodium activation on the dynamics of AP initiation, the model was, in addition, modified in the following ways:

- The sodium peak conductance $\bar{g}_{N a}$ was changed.

- The width of the activation curve was decreased.

- The $m(t)^{3}$-term describing the activation of the sodium channels was replaced by the sodium activation curve measured by Huguenard et al. (1989), with two parameters $k$ and $V_{1 / 2}$ :

$$
I_{N a}=\bar{g}_{N a}\left(1+\exp \left(-\left(V+V_{1 / 2}\right) / k\right)\right)^{-1} h\left(V-E_{N a}\right)
$$

Several combinations of $k, V_{1 / 2}$ and $\bar{g}_{N a}$ were used to change the AP onset rapidness while preserving a stationary firing rate of $10 \mathrm{~Hz}$ in the entire regime where the model was able to generate APs.

\section{Model B}

To assess if adaptation currents have an impact on AP initiation in vivo, I implemented a second model, developed by Wang et al. (2003), which also includes adaptation currents. The model has 
two compartments, one representing the soma and the initial segment $\left(V_{s}\right)$ and the other representing the dendritic tree $\left(V_{d}\right)$. The AP generating currents are located in the soma. High threshold $\mathrm{Ca}^{2+}$ as well as $\mathrm{Ca}^{2+}$-dependent $\mathrm{K}^{+}$currents are present in both compartments. In addition, the somatic compartment includes slow $\mathrm{Na}^{+}$-activated $\mathrm{K}^{+}$channels.

The dynamics of the model is defined by the following differential equations:

$$
\begin{aligned}
C_{m} \frac{d V_{s}}{d t}=-I_{L}-I_{N a}-I_{C a, s}-I_{K C a}-\left(g_{c} / p\right)\left(V_{s}-V_{d}\right)+I_{e x t}(t) \\
\frac{d h}{d t}=\phi_{h}\left(\alpha_{h}\left(V_{s}\right)(1-h)-\beta_{h}\left(V_{s}\right) h\right) \\
\frac{d n}{d t}=\phi_{n}\left(\alpha_{n}\left(V_{s}\right)(1-n)-\beta_{n}\left(V_{s}\right) n\right) \\
\frac{d\left[C a^{2+}\right]_{s}}{d t}=-\alpha_{C a, s} I_{C a, s}-\left[C a^{2+}\right]_{s} / \tau_{C a, s} \\
\frac{d\left[N a^{+}\right]}{d t}=-\alpha_{N a} I_{N a}-3 R_{\text {pump }}\left(\varphi_{N a}\left(\left[N a^{+}\right]_{e q}\right)\right) \\
C_{m} \frac{d V_{d}}{d t}=-I_{L}-I_{C a, d}-I_{K C a, d}-\left(g_{c} /(1-p)\right)\left(V_{d}-V_{s}\right) \\
\frac{d\left[C a^{2+}\right]_{d}}{d t}=-\alpha_{C a, d} I_{C a, d}-\left[C a^{2+}\right]_{d} / \tau_{C a, d}
\end{aligned}
$$

The membrane capacity is denoted by $C_{m}=1 \mu \mathrm{F} / \mathrm{cm}^{2}$, the external current by $I_{\text {ext }}(t)$ and the leak current by $I_{L}=g_{L}\left(V-V_{L}\right)$. The coupling current between the soma and the dendrite is proportional to $V_{d}-V_{s}$, with the coupling conductance $g_{c}=2 \mathrm{mS} / \mathrm{cm}^{2}$. The ratio of the somatic to the total surface area of the neuron is given by $p=0.5$.

The peak conductance densities are given by $g_{L}=0.1, g_{N a}=45, g_{K}=18, g_{C a, s}=g_{C a, d}=1, g_{K C a, s}=$ $g_{K C a, d}=5$ and $g_{K N a}=5 \mathrm{mS} / \mathrm{cm}^{2}$. The reversal potentials are given by $E_{L}=-65, E_{N a}=55, E_{K}=-80$ and $E_{C a}=120 \mathrm{mV}$. The sodium current in the somatic compartment is given by:

$$
I_{N a}=g_{N a} m_{\infty}^{3}\left(V_{s}\right) h\left(V_{s}-E_{N a}\right),
$$

with $m_{\infty}\left(V_{s}\right)=\alpha_{m}\left(V_{s}\right) /\left(\alpha_{m}\left(V_{s}\right)+\beta_{m}\left(V_{s}\right)\right), \alpha_{m}(V)=-0.1(V+33) \exp (0.1(V+33))$ and $\beta_{m}(V)=$ $4 \exp (-(V+58) / 12)$. The inactivation variable $h$ is described by $\alpha_{h}(V)=0.07 \exp (-(V+50) / 10)$ and $\beta_{h}(V)=(\exp (-0.1(V+20)+1)$. The delayed rectifier current density is given by:

$$
I_{K}=g_{K} n^{4}\left(V_{s}-E_{K}\right),
$$

with the activation variable $n$ given by $\alpha_{n}(V)=-0.01(V+34) /(\exp (-0.1(V+34))-1)$ and $\beta_{n}=$ $0.125 \exp (-(V+44) / 25)$. The temperature factors are $\phi_{h}=\phi_{n}=4$. The time constants are $\tau_{x}(V)=$ $\left(\alpha_{x}(V)+\beta_{x}(V)\right)^{-1}$. The high-threshold calcium current is defined as:

$$
I_{C a}=\bar{g}_{C a} v_{\infty}^{2}(V)\left(V-E_{C a}\right),
$$

with the activation variable $v_{\infty}(V)=(1+\exp (-(V+20) / 9))^{-1}$. The voltage-independent, calcium 
activated potassium current is given by:

$$
\left.I_{K C a}(V)=\bar{g}_{K C a}\left(\left[\mathrm{Ca}^{2+}\right]_{i} /\left(\left[\mathrm{Ca}^{2+}\right]_{i}+K\right) D\right)\right)\left(V-V_{K}\right),
$$

with $K_{D}=30 \mu \mathrm{M}$. The intracellular calcium concentration $\left[\mathrm{Ca}^{2+}\right]$ is assumed to be governed by a linear equation with $\alpha_{\mathrm{Ca}}$ proportional to the ratio of the membrane and the volume immediately beneath the membrane, $\alpha_{C a}=2 \cdot 10^{-3} \mu \mathrm{M}(\mathrm{ms} \mu \mathrm{A})^{-1} \mathrm{~cm}^{-1}$ in the dendritic compartment and $\alpha_{C a}=$ $6.7 \cdot 10^{-4} \mu \mathrm{M}(\mathrm{ms} \mu \mathrm{A})^{-1} \mathrm{~cm}^{-1}$ in the somatic compartment. The extrusion and buffering processes are described collectively by a first-order decay process with a time constant $\tau_{C a}=80 \mathrm{~ms}$ in the dendrite and $\tau_{\mathrm{Ca}}=240 \mathrm{~ms}$ in the soma. The intracellular $\left[\mathrm{Na}^{+}\right]_{i}$ concentration is incremented by $\mathrm{Na}^{+}$influx through $\mathrm{I}_{\mathrm{Na}}$. The $\mathrm{Na}^{+}$-dependent $\mathrm{K}^{+}$-current is determined by:

$$
I_{K N a}=\bar{g}_{K N a} \omega_{\infty}\left(\left[N a^{+}\right]_{i}\right)\left(V_{s}-E_{K}\right),
$$

with the activation function $\omega_{\infty}\left(\left[N a^{+}\right]_{i}\right)=P_{\max } /\left(1+\left(E C_{50} /\left(\left[N a^{+}\right]_{i}\right)\right)^{n_{H}}\right) . P_{\max }=0.37$ defines the maximum opening probability of the channels, $E C_{50}=38.7 \mathrm{mM}$ is the $\left[\mathrm{Na}^{+}\right]_{i}$ for half activation and $n_{H}=3.5$ is the Hill coefficient. The influx of $\left[\mathrm{Na}^{+}\right]_{i}$ is controlled by $-\alpha_{N a} I_{N a}$ with $\alpha_{N a}=3 \cdot 10^{-4} \mathrm{mM}$. The extrusion of $\left[\mathrm{Na}^{+}\right]_{i}$ by the ion pump was modeled as $-3 R_{\text {pump }}\left(\varphi_{\mathrm{Na}}\left(\left[\mathrm{Na}^{+}\right]_{i}\right)-\varphi\left(\left[\mathrm{Na}^{+}\right]_{e q}\right)\right)$, where $\varphi_{N a}(x)=x^{3} /\left(x^{3}-K_{p}^{3}\right), K_{P}=15 \mathrm{mM}$ and $R_{p u m p}=6 \cdot 10^{-4} \mathrm{mM} / \mathrm{ms}$. The sodium concentration at the resting state is given by $\left[\mathrm{Na}^{+}\right]_{e q}=8 \mathrm{mM}$.

In this model, a fluctuating synaptic current was injected using the same point conductance model, and was subsequently modified in the same way as Model A. In both models, $7 \mathrm{~s}$ long periods of neuronal activity were simulated. In the second model, care was taken that the adaptation currents where statistically stationary.

\subsection{Preprocessing of in vivo and in vitro recordings}

All in vivo and in vitro intracellular recordings were made by Maxim Volgushev at the Ruhr-University in Bochum. The details of the surgery and the maintenence of the animals are described in (Volgushev et al., 2000).

I analyzed data from 31 cells recorded in vivo. For each cell, responses to the presentation of moving gratings of different orientation (duration $5-7 \mathrm{~s}$ ), and periods of spontaneous activity $(10-120 \mathrm{~s}$ ) were recorded. Altogether, 266 in vivo recordings were analyzed. For comparison I also examined data from 16 cells recorded in slices (11 in rat, 3 in mouse, 2 in cat), resulting in 70 in vitro recordings.

Recordings were subsequently chosen such that in each recording at least 5 APs were generated. Special care was taken that the MP and the peak amplitude of APs fulfilled a stationarity criterion. With this stationarity test, cells which exhibited slow drifts in their subthreshold MP or decaying AP amplitudes were excluded, which could indicate intrinsic changes in the cellular excitability, drifts in the recorded voltage or dying cells. The stationarity of the recordings was tested by computing the standard deviation of the MP, the AP peak potentials and the onset potentials for increasing window sizes ranging from $100 \mathrm{~ms}$ to $7 \mathrm{~s}$. A recording was classified as being stationary if the average deviation in peak amplitude and AP onset potential varied less than $10 \%$ for all window sizes. A typical outcome of the stationarity test is shown in Fig. 4.1. Typically, the variability decreases slightly with decreasing width of the window. It is important to note that this decrease does not necessarily reflect non-stationarities in the recordings. Some channel properties, such as long lasting adaptation, 


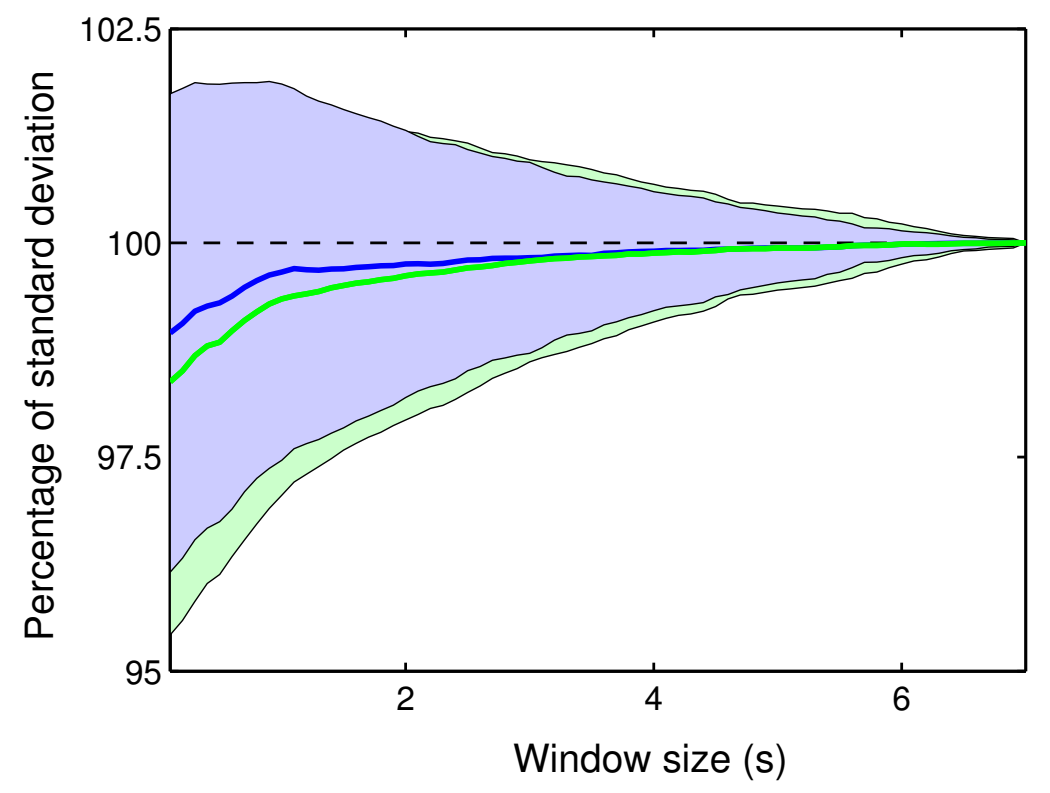

Figure 4.1: Stationarity test of AP peak and onset potentials. For increasing window sizes, ranging from $100 \mathrm{~ms}$ up to $7 \mathrm{~s}$, the average standard deviation (thick solid lines) was determined for the AP peak (green) and onset potential (blue). The filled areas denote the mean \pm standard deviation. They are determined relative to the values of a window size of $7 \mathrm{~s}$. Only traces for which the average standard deviation differed less than $10 \%$ for all window sizes were taken into account for a further analysis. 
may well operate on time scales of about 1 s (Henze \& Buzsáki, 2001). All selected recordings were subsequently interpolated to a resolution of $\Delta t=10 \mu \mathrm{s}$.

\subsection{Phase-plane representation of action potential dynamics}

\subsubsection{Embedding of membrane potential recordings}

A tool that is often used in the study of nonlinear systems is a phase plane representation of the dynamics. In this representation, two dynamical variables are plotted as a function of each other. In the Hodgkin-Huxley equations, this could be for example the membrane potential $V(t)$ and the activation variable of the delayed rectifier $n(t)$. In experiments, it is usually not possible to measure the dynamical channel kinetics of a single channel together and the dynamics of the MP at the same time during the course of an AP. From the theory of time-series analysis it is, however, known that the dynamics of a generic non-linear system can be reconstructed by embedding a trace of some observable, e.g. $V(t)$ in a delay-time vector space (Takens, 1981; Kantz \& Schreiber, 1997). The canonical choice for an $n$-dimensional embedding is given by:

$$
x(t)=(V(t), V(t-\tau), \ldots, V(t-(n-1) \tau))^{T} \in \mathbb{R}^{n},
$$

with the embedding time-lag $\tau$. The dimensionality $n$ is here a free parameter. To recover the complete dynamics of the underlying dynamical system, $n$ has to be deduced from dimensionality analysis (Grassberger \& Procaccia, 1983).

For the following analysis, I will use an embedding space of dimension 2, which allows to graph the resulting dynamics in a 2D space. A drawback of the standard delay-vector embedding is that if $\tau$ is not very large, the two vector components can be expected to be highly correlated if the underlying dynamics is sufficiently smooth and all embedding-vectors will therefore lie very close to the diagonal. Thus, a different embedding is chosen, in which the MP and the numerical first derivative, i. e. the velocity of the MP, are combined in a vector:

$$
x(t)=(V(t),(V(t+\tau)-V(t-\tau)) /(2 \tau))^{T},
$$

where the time $\tau$ is here chosen as the sampling interval of a recording. The construction of this embedding obviously requires only a linear transformation from the standard delay-time vector embedding discussed above.

This particular choice of embedding has several advantages. Most importantly, the two embedding dimensions have a direct intuitive interpretation as they represent the MP and its rate of change. Secondly, the two dimensions are uncorrelated if the dynamics is stationary:

$$
\left\langle V(t) \frac{d}{d t} V(t)\right\rangle=\frac{1}{2} \frac{d}{d t}\left\langle V(t)^{2}\right\rangle=0,
$$

where the brackets $\langle\cdot\rangle$ denote ensemble averages. Thus, deviations from stationarity are directly visible in a phase-plot. Thirdly, in most neuron models, the dynamics of an AP is confined in a two-dimensional sub-manifold of the state-space resulting phase plots can be directly compared with the predictions from the Hodgkin-Huxley theory, as the dynamics of the membrane potential $V(t)$ in Eq. (2.3) is formulated as a first-order differential equation. 


\subsection{AP initiation in cortical neurons}

\subsubsection{Fast onset dynamics}

We now have all tools at hand to compare the AP dynamics which is predicted by Hodgkin-Huxley type models with the AP initiation dynamics of cortical neurons. Representative examples of APs recorded in cats' visual cortices are displayed in Fig. 4.2A,C. These were acquired in vivo and in vitro. In the in vitro recordings, current steps were injected via a patch-pipette. To make sure that only intrinsic, ion channel gated currents through the membrane were involved in the generation of an AP, the amplitude of the injected step current was carefully adjusted to values close to the rheobase of the neuron.

In the MP traces, the dynamics of the AP is characterized by a rapid depolarization with peak potentials around $+10 \mathrm{mV}$. The half width at half height $(\mathrm{HWHH})$ is approximately $1 \mathrm{~ms}$ in the in vivo recording and a bit larger, approximately $1.5 \mathrm{~ms}$, in the in vitro recording. Fig. 4.2E shows, for comparison, a trace of a simulated AP (Model A). The simulated AP shares characteristic features of the measured APs, i.e. a comparable duration and a sign reversal at peak depolarization. However, several differences compared to the measured APs are apparent. Firstly, the peak depolarization is larger in the simulation, indicating a different balance between sodium channel activation, inactivation and activation of the delayed potassium rectifier. Secondly, the simulated AP exhibits a pronounced afterhyperpolarization, which is almost missing in the measured APs.

The most striking difference is, however, the AP onset dynamics. While the cortical APs exhibit a kink-like AP initiation, the simulated AP exhibits a very smooth onset behavior. This difference stands out much more clearly in the corresponding phase-plots (Fig. 4.2B,D,F). There, the kink-like onset behavior manifests itself as a virtually vertical take-off of $V-d V / d t$-trajectories at AP onset. At the start of the AP loops, the velocity increases rapidly from values less than $5 \mathrm{mV} / \mathrm{ms}$ to more than $20 \mathrm{mV} / \mathrm{ms}$. This several-fold increase of the velocity occurs within a range of less than $1 \mathrm{mV}$ and takes less than $0.2 \mathrm{~ms}$. This dynamical feature is shared by both in vivo and in vitro recordings. In contrast, the simulated AP exhibits a very smooth onset dynamics, in which a velocity of $20 \mathrm{mV} / \mathrm{ms}$ is reached over a voltage range of $7-8 \mathrm{mV}$ after about $1 \mathrm{~ms}$.

In Fig. 4.3A-D, phase plots and MP traces of recordings from two neurons with different receptive field types from cat visual cortex are displayed. In both recordings, the neurons were visually stimulated with moving gratings of preferred orientation. The first cell exhibits a simple receptive field. This is reflected by a pronounced modulation of its subthreshold MP and a clearly modulated rate response (Fig. 4.3B). The second cell exhibits a complex receptive field. Although it fires at a high rate of approximately $10 \mathrm{~Hz}$, the MP does not lock to the phase of the moving grating (Fig. 4.3D). In Fig. 4.3A,C, the corresponding phase-plots are displayed. All APs initiate as rapidly as in Fig. 4.2B.

In Fig. 4.3E, a simulated trace of a conductance-based model $\mathrm{A}$ is shown. To model the in vivo fluctuations of the MP, a fluctuating synaptic input was added to the model equations, where the parameters values were adjusted such that the resulting MP fluctuations match the typical stationary MP fluctuations of a cortical neuron in vivo. As for the cortical neuron with a complex receptive field, APs are emitted irregularly with a stationary rate of approximately $10 \mathrm{~Hz}$. The AP onset behavior is, however, as before, very different from the onset dynamics of the cortical neurons. All APs initiate very slowly over a large voltage range, whereas in both simple and complex cells APs rise vertically out of the cloud of subthreshold fluctuations. 

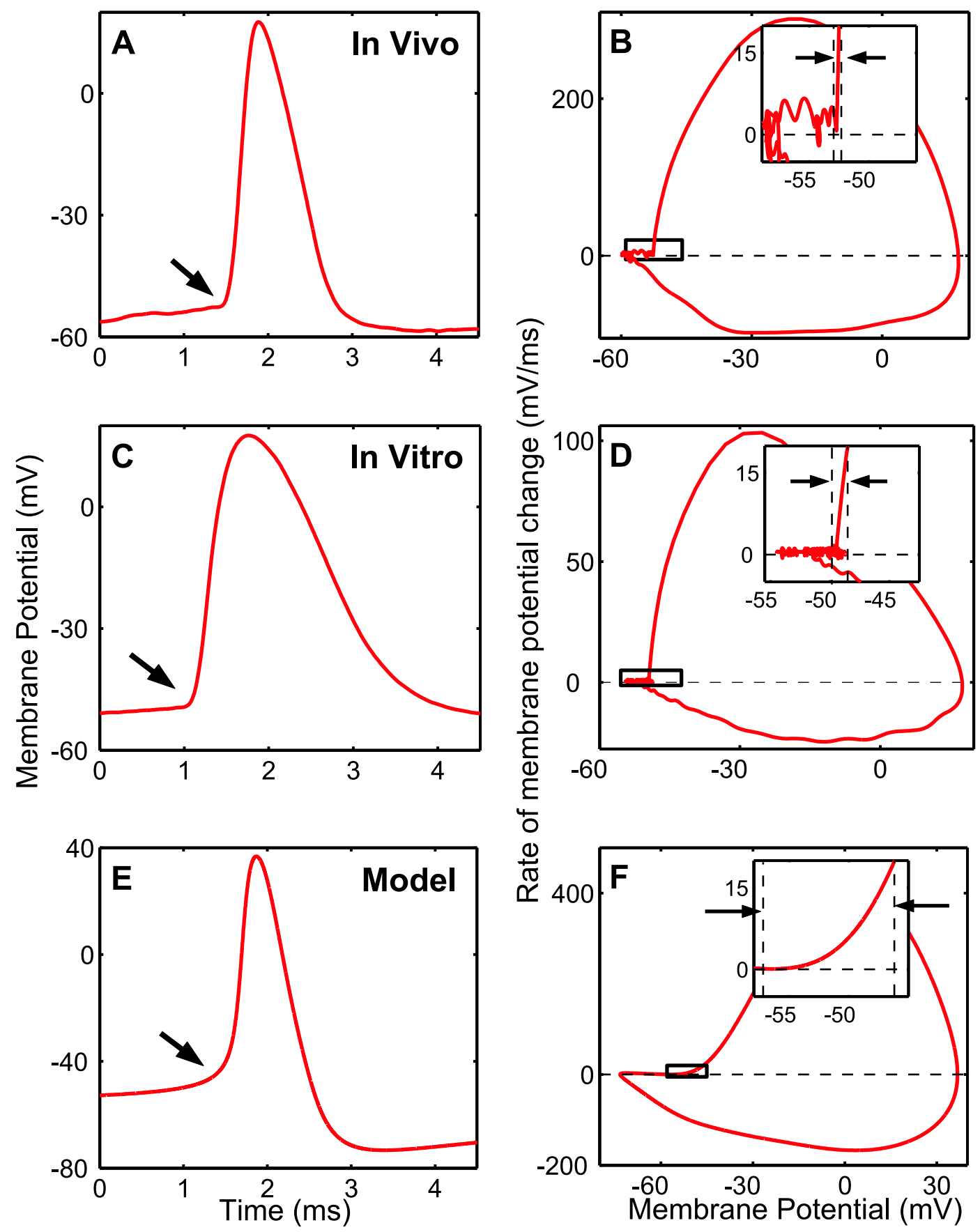

Figure 4.2: Dynamics of AP initiation in neocortical neurons and in a Hodgkin-Huxley type conductance-based model of a neocortical neuron. (A) Plot of an AP recorded in cat visual cortex in vivo. The arrow denotes the characteristic kink at the AP onset. (B) Phase plot (dV/dt vs. V) of the same AP as in (A). The big loop corresponds to the AP. Inset: Initial phase of AP at expanded scale. The AP onset manifests itself as an almost vertical take-off. (C,D) AP recorded in vitro (slice of cat visual cortex, $20^{\circ} \mathrm{C}$ ), in the same representation as in (A,B). Although the AP is broader, and the peak velocity smaller, the AP onset dynamics remains fast, similar as in $(A, B)$. (E,F) Typical shape of an AP from a simulation of a Hodgkin-Huxley type conductance-based model of a neocortical neuron. Note the smooth AP onset in (E) in comparison to (A) and (C), which is reflected by the slow increase of the rate of MP change in $(F)$ in comparison to $(B)$ and $(D)$. 

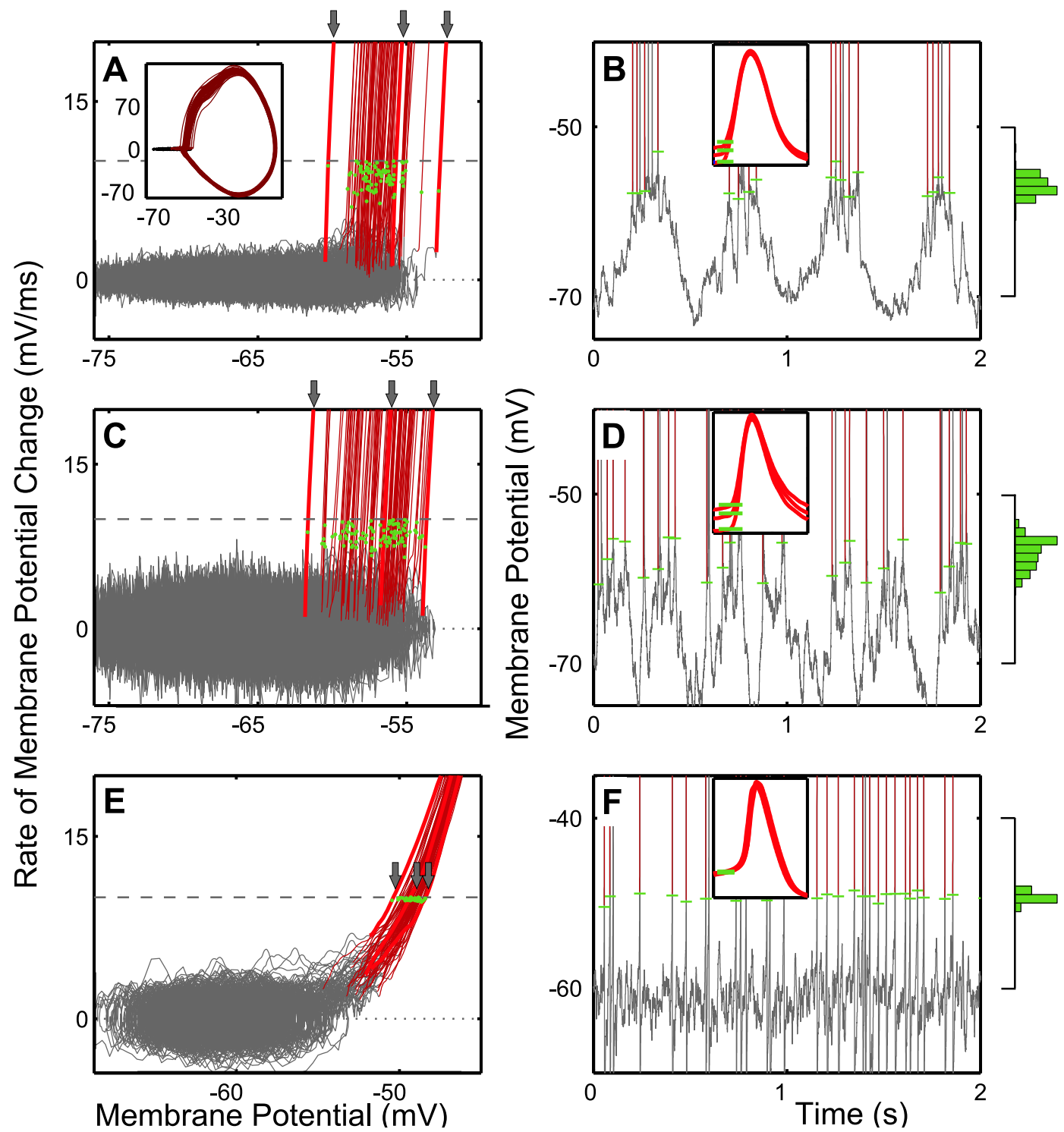

Figure 4.3: Different AP initiation in visual cortex neurons recorded in vivo and in a Hodgkin-Huxley type model subject to synaptic fluctuating input. (A) Response of a neuron with a simple receptive field to a moving grating of optimal orientation represented by a phase plot. For better resolution, only the initial phases of the APs together with subthreshold fluctuations (dark grey) are shown in the main panel. The inset shows the complete trace. APs are shown in red, three sample APs are marked by arrows. Green dots mark the data point immediately before crossing the threshold velocity $(10 \mathrm{mV} / \mathrm{ms})$. (B) Part of the recording shown in (A) as a voltage trace (APs truncated). Green bars: AP onset potentials. Inset: Three sample APs marked in (A). Histogram on the right hand side: Distribution of AP onset potentials in the entire 7s recording. Color code as in (A). (C,D) Response of a neuron with a complex receptive field to a moving grating of optimal orientation. Color code and trace representations as in (A,B). (E,F) Response of a Hodgkin-Huxley type conductance-based model of a neocortical neuron subject to fluctuating synaptic input. Color code and trace representations as in $(A, B)$.

Note the steeper upstroke and larger variability of the onset potentials in the recorded cells compared to the simulation. 


\subsubsection{Large variability}

The phase plots in Fig. 4.3 also display a second salient feature of cortical APs: The onset potentials vary strongly, covering in some instances more than $\Delta V=10 \mathrm{mV}$ (marked by green bars) without an overall trend. Because the rate of change of the MP always rises very abruptly at AP onset, the onset potential of an AP could be reliably estimated as the potential at which the MP rate of change $d V / d t$ reaches a value of $10 \mathrm{mV} / \mathrm{ms}$.

As apparent in Fig. 4.3E,F, also the simulated trace exhibits variable AP onset potentials. The range is, however, much smaller and reaches only values of $\Delta V=3 \mathrm{mV}$. There are thus two features which render the cortical AP initiation distinctly different from APs of Hodgkin-Huxley type models: Firstly, the initial AP phase is approximately 10 times faster in the recorded neurons compared to the conductance-based models. Secondly, the onset variability is approximately 3 times larger in the recorded cells.

So far, I did not specify in detail how the parameters of the Hodgkin-Huxley type model were chosen to mimic the dynamics of cortical neurons. The natural question that arises is: Can the parameters in Hodgkin-Huxley type models in principle be adjusted, such that the dynamics of AP initiation reflects the AP initiation dynamics of cortical neurons? In the following section I will argue that the two observed features of cortical AP initiation are mutually exclusive in the class of Hodgkin-Huxley type models.

\subsection{Two antagonistic dynamical features in cortical AP initiation}

What determines the AP onset dynamics in the class of Hodgkin-Huxley type models? As discussed in Sec. 2.6, the initial phase of an AP is determined by the activation of voltage-dependent sodium channels. There, the opening of sodium channels was described by the sodium activation curve and the dynamics of the associated gating variables:

$$
C_{m} \dot{V}(t)=\bar{g}_{\mathrm{Na}} h(t) m(t)^{3}\left(E_{\mathrm{Na}}-V(t)\right)+I_{\mathrm{K}}+\cdots
$$

As before, $C_{m}$ denotes the membrane capacity, $\bar{g}_{\mathrm{Na}}$ the sodium channel peak conductance, $m(t)$ the sodium channel activation and $h(t)$ the inactivation. The activation and inactivation follow a first order kinetics, where the time constant of the activation $\tau_{m}(V)$ is typically about $0.2 \mathrm{~ms}$. The inactivation constant $\tau_{h}$, as well as the time constants of all other voltage-gated channels are typical much larger of the order of $10 \mathrm{~ms}$.

During the initial AP phase, an upper bound of the MP rate of change is thus given by replacing $m(t)$ by its steady state value $m_{\infty}(V)$ and replacing the inactivation variable, as well as all other channel currents by constants:

$$
\dot{V} \leq I_{0} / C_{m}+\bar{g}_{\mathrm{Na}} h_{0} m_{\infty}^{3}(V)\left(E_{\mathrm{Na}}-V(t)\right) / C_{m}
$$

Here $h_{0}$ denotes the constant amount of sodium channel inactivation and $I_{0}$ is the current flowing through other channels including the leak current.

This upper bound on the AP rate of change establishes a direct link between the activation curve and the AP onset dynamics. It further implies that in the Hodgkin-Huxley framework the AP onset dynamics can be modified in two ways: 
- By changing the shape of the sodium channel activation curve $m_{\infty}^{3}(V)$.

- By changing the effective sodium channel peak conductance $\bar{g}_{\mathrm{Na}} h_{0}$.

How does the AP onset dynamics depend on the shape of the sodium channel activation curve and the effective sodium peak conductance? The influence of these two factors on the AP onset dynamics is illustrated in Fig. 4.4. Using an experimentally obtained activation curve from patches of cortical neurons (Huguenard et al., 1988; Colbert \& Pan, 2002), results in a shallow AP onset (Fig. 4.4A). The AP onset can be steepened by using a hypothetical activation curve with an increased steepness. However, even with a five-fold increase in steepness, the simulated APs do not rise as fast as the recorded APs in Fig. 4.2.

What happens when the effective sodium channel peak conductance is changed? In Fig. 4.4B, it is illustrated that an increased peak conductance leads the onset steepness unaffected and shifts the AP onset potentials. The peak conductance $\bar{g}_{\mathrm{Na}}$ of sodium channels is determined by the number of channels in the membrane and can thus be assumed to be fixed. The inactivation of sodium channels, however, is an important mechanism which scales the effective peak conductance. Varying levels of sodium channel inactivation are thus a suitable candidate mechanism for explaining the observed variability of onset potentials (Azouz \& Gray, 2000; Henze \& Buzsáki, 2001). Thus, the straightforward approach to model the AP initiation of cortical neurons with the Hodgkin-Huxley formalism is to combine a steep activation curve with varying values of the inactivation variable $h_{0}$.

In Fig. 4.4C,D, the impact of inactivation on the onset potential variability is visualized for different shapes of the sodium channel activation curve. For steep activation curves, a 4-fold change in the peak conductance leads only to a small variability of the AP onset potentials (Fig. 4.4C). On the other hand, for broader activation curves, which result in a slow AP onset, the same 4-fold change in the peak conductance leads to a larger onset potential variability (Fig. 4.4D).

This antagonistic behavior in onset steepness and variability can be derived by a straightforward calculation. Right at AP onset, the sodium activation curve starts exponentially:

$$
m^{3}(V)=\left(1+\exp \left(-\left(V-V_{1 / 2}\right) / k\right)\right)^{-3} \stackrel{V \ll V_{1 / 2}}{=} \exp \left(3\left(V-V_{1 / 2}\right) / k\right)
$$

A multiplication of $m^{3}(V)$ by a factor $G$ is therefore equivalent to a shift of the whole curve by $k \log G$. For measured values of $k \approx 5$, a shift of $10 \mathrm{mV}$ would therefore require a 7-fold change in the effective peak conductance. Consequently, for a steep activation curve with $k=1$, the same shift would require a 22000-fold change in sodium channel inactivation, which clearly is physiologically unplausible.

To quantitatively compare the AP onset dynamics in the recordings with the AP dynamics in HodgkinHuxley type models, Fig. 4.5 shows a plot in which the AP onset span is graphed against the AP onset rapidness for real and simulated recordings. The onset rapidness was determined as follows: For each $\mathrm{AP}$, a line was fitted to the AP curve in the phase plane representation at $d V / d t=10 \mathrm{mV} / \mathrm{ms}$. The onset rapidness of a single AP is defined as the slope of this line. The onset rapidness of a recording is then given as the mean onset rapidness of all APs in the recording. The onset span of a recording is a measure of the AP onset variability. It is defined as the difference between the maximum and the minimum voltage at which APs started in the recording.

In the simulations, both models (Model A and B) were used, in which fluctuating synaptic currents were injected. The data points of the model simulations reflect the antagonism between the onset span and rapidness: Simulated APs either exhibited a large onset rapidness or a large onset span, but never 
A
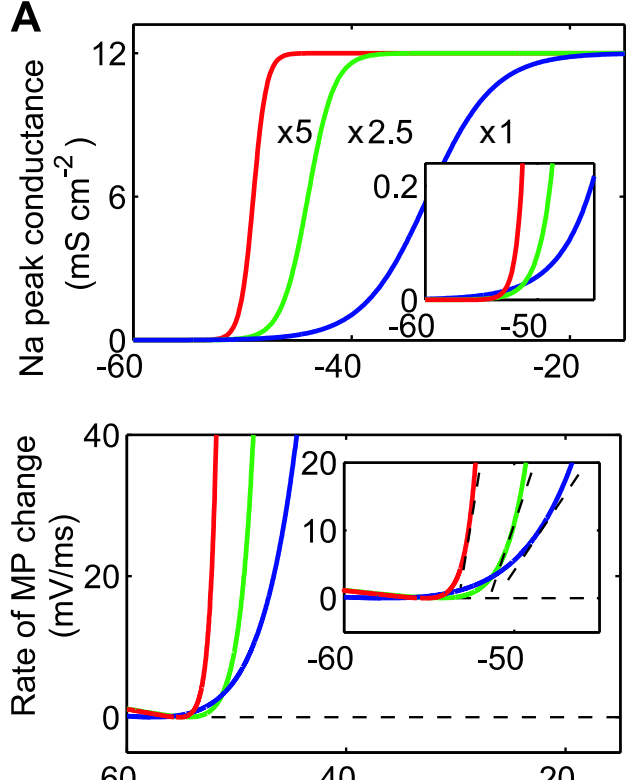

C
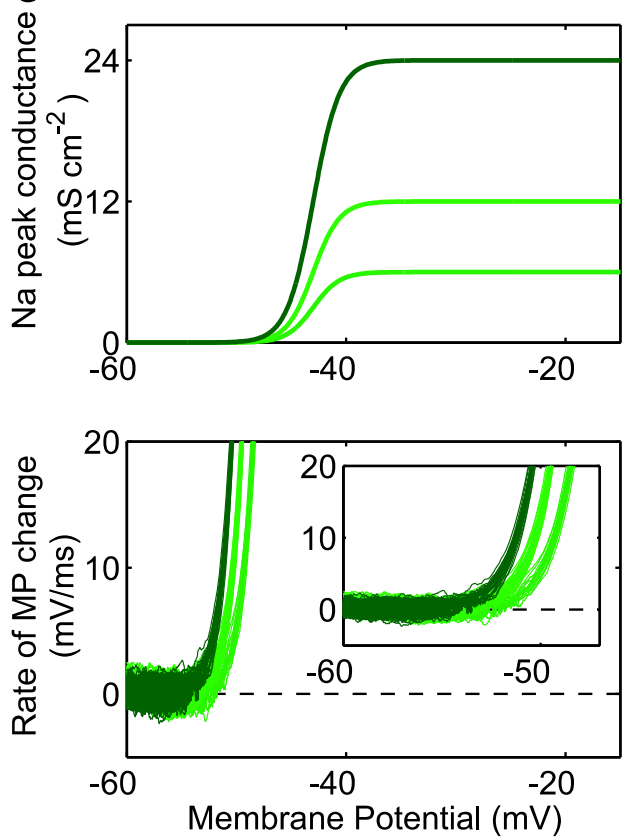

B
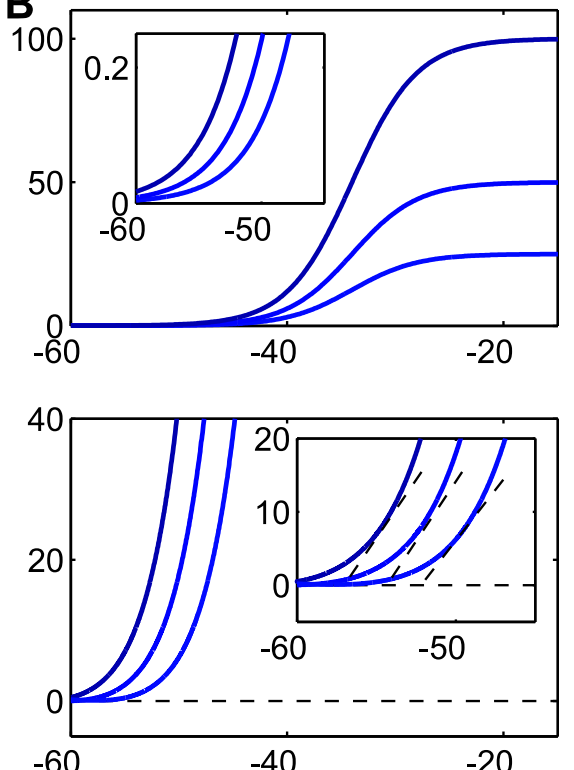

D
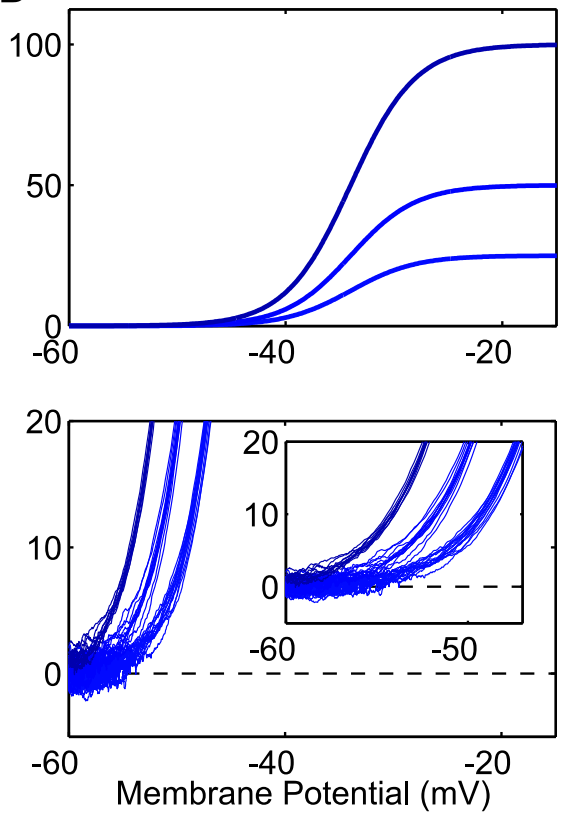

Figure 4.4: Impact of different shapes of the sodium activation curve and peak conductance on the AP initiation in a conductance-based model neuron. (A) An increase of the steepness of the sodium channel activation curves (upper panel) leads to a faster up-stroke of APs (lower panel). Corresponding curves are marked by the same color. Insets: Magnification of activation curves and AP onset, respectively. (B) A change in the sodium peak conductance (upper panel) leads to a shift of the onset potential (lower panel), but has little effect on the onset slope of an AP. Use of color as in $(A)$.

(C, D) Initiation of APs with steep (C) and shallow (D) activation curves and different effective sodium peak conductances driven by fluctuating synaptic input. For each activation curve, several simulated action potentials are superimposed. With a steep activation curve of sodium channels, a five-fold change of the effective peak conductance leads to a much smaller span of onset potentials (C: $2.5 \mathrm{mV})$ than for a shallow activation curve (D: $6 \mathrm{mV})$. Use of color and insets as in (A). 


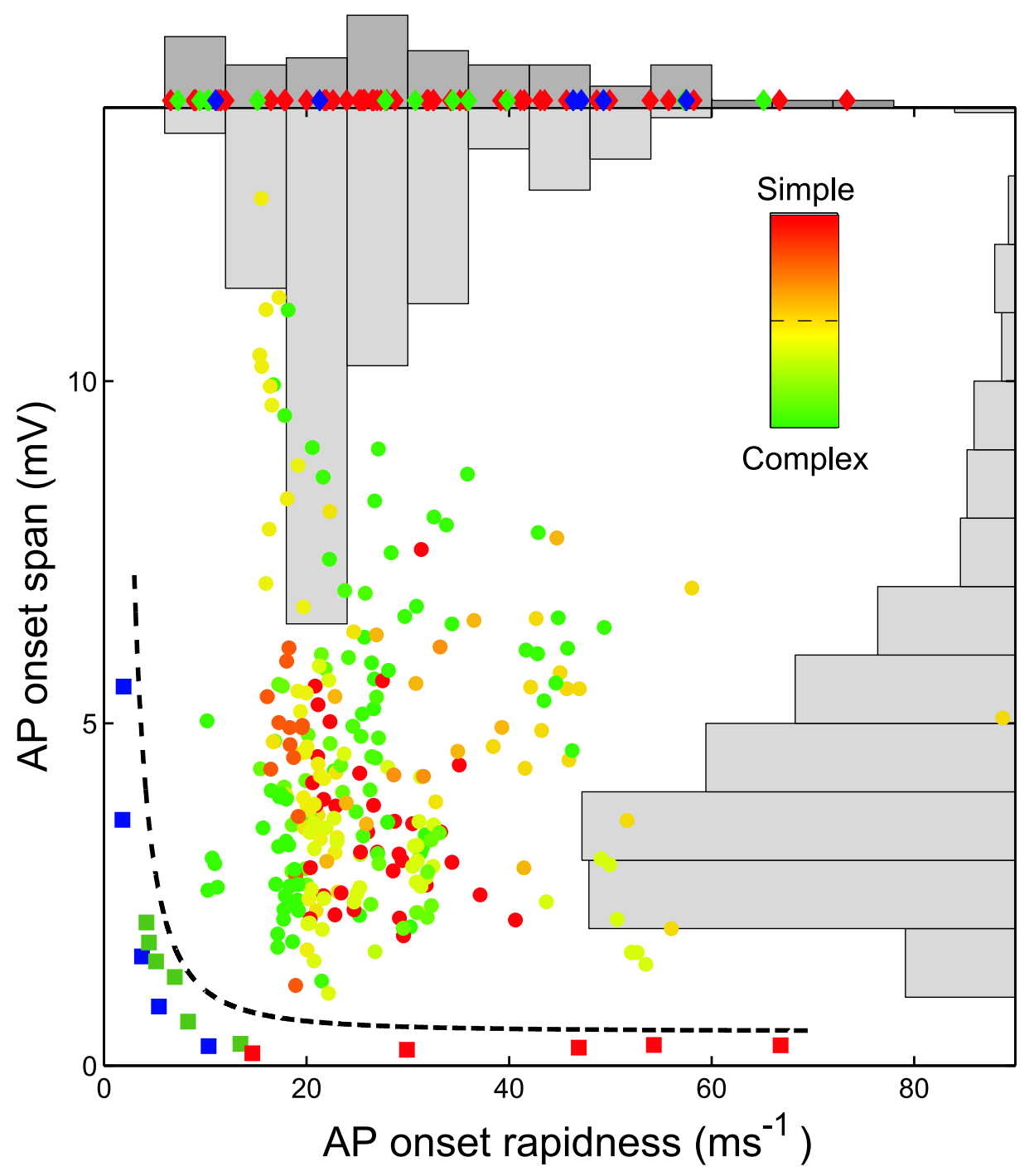

Figure 4.5: Discrepancy in AP initiation between cortical neurons and Hodgkin-Huxley type conductance-based neuron models.

Scatter plot of the AP onset variability vs. the onset rapidness (see main text). Dots: Recordings from cat visual cortex neurons in vivo, color coded for the simple/complex index, green corresponding to 0 (complex cells) and red corresponding to 2 (simple cells). Diamonds (top of the panel): In vitro data (blue: cat visual cortex; green: rat visual cortex: red: mouse hippocampus). Squares: Simulation results from two different conductance-based models driven by fluctuating synaptic input. In each model, a set of activation curves of sodium channels with different steepness and different peak conductance were used (Blue: Model A, Green: Model B, Red: Model A with a steep activation curve). The dashed line separates the experimental data from the simulation results. The histograms show the marginal distributions of the in vivo data (light gray inside the panel) and of the in vitro data (dark gray on top of the panel). 
both. The data points obtained from the simulations are clearly separated from the data points of the in vivo recordings. I also evaluated the AP onset span for in vitro recordings. Their distribution of onset rapidness was very similar to the distribution obtained from the in vivo recordings (Fig. 4.5, histogram on top).

How typical is the rapid onset dynamics in cortical neurons? Does it occur only during evoked activity or is it also present under spontaneous ongoing activity? It turns out that the onset of APs is a very robust phenomenon which occurs in neurons from:

- cat visual cortex in vivo and in vitro

- cat prefrontal cortex in vivo

- rat visual cortex in vitro

- mouse hippocampus in vitro

- mitral cells from the olfactory bulb of zebrafish in vivo

In invertebrate neurons, the picture is more involved. AP recordings from snail neurons of different cells exhibit a large variability of onset speeds. The onset dynamics is in most cases, however, well fit by a standard sodium activation curve.

\subsection{Three hypotheses to explain the anomalous AP initiation in cortical neurons}

What could be the biophysical mechanisms that enables cortical APs to initiate much faster and at the same time with a much larger variability than predicted by the Hodgkin-Huxley theory? Apparently, a basic assumption underlying the AP initiation in cortical neurons seems to be violated qualitatively. In the following, three candidate mechanisms are presented to explain the anomalous AP initiation dynamics in cortical neurons.

\subsubsection{Ion channel stochasticity}

A basic assumption of the Hodgkin-Huxley theory is that the ensemble dynamics of voltage-gated ion channels can be described by products of activation and inactivation variables which, in turn, are modeled as first order kinetics. Strictly, this assumptions is, however, only valid within the limit of an infinite number of channels in the membrane, where each channel carries an infinitesimal current. Neuronal membranes, however, naturally exhibit only a limited number of voltage-gated channels, and the current which flows through an individual channel switches between discrete values (See Fig. 4.6).

This discrete switching will naturally cause discrete changes in the total current flowing through the neuronal membrane. Can this switching explain the anomalous AP initiation observed in cortical neurons? To answer this question, it is important to first assess how many channels on average are involved in the generation of an AP. The conductance of a single voltage-gated Na-channel is approximately given by $\gamma=20 \mathrm{pS}$ (Neumcke \& Stämpfli, 1982) (Note: The single channel conductance 


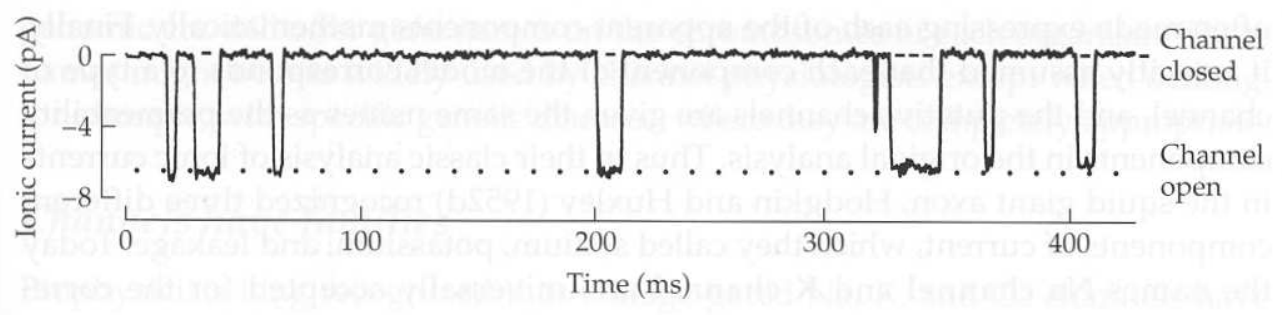

Figure 4.6: Ionic current flowing through a single voltage-gated channel. The channel opens and closes stochastically, the current which flows through the channel switches thereby from 0 to -6.6 pA. (Adapted from Sanchez et al. (1986))

estimate can differ up to a factor of 2 , depending on the recording method and conditions). From the total sodium current flowing through a patch of the membrane, the number of channels in the membrane of a pyramidal cell can be estimated as approximately $N=12000$, yielding a total conductance of $240 \mathrm{nS}$. If all channels are assumed to open and close independently, the fluctuations of the conductance are of order $\sigma_{\gamma}=\sqrt{N} / N \bar{g}_{\mathrm{Na}} \approx 10^{-2} \bar{g}_{\mathrm{Na}}$. To assess if this magnitude of intrinsic channel noise can account for the anomalous AP initiation in cortical neurons, I implemented a discrete Markov model, in which the opening and closing of individual sodium and potassium channels was simulated explicitly (Schneidman et al., 1998). The simplest kinetic model for a population of sodium channels is given by the following scheme (Patlak, 1991):

$\begin{array}{ccccccc} & 3 \alpha_{m} & & 2 \alpha_{m} & & \alpha_{m} & \\ m_{0} h_{1} & \rightleftharpoons & m_{1} h_{1} & \rightleftharpoons & m_{2} h_{1} & \rightleftharpoons & m_{3} h_{1} \\ & \beta_{m} & & 2 \beta_{m} & & 3 \beta_{m} & \\ \alpha_{h} \uparrow \downarrow \beta_{h} & & \alpha_{h} \uparrow \downarrow \beta_{h} & & \alpha_{h} \uparrow \downarrow \beta_{h} & & \alpha_{h} \uparrow \downarrow \beta_{h} \\ & 3 \alpha_{m} & & 2 \alpha_{m} & & \alpha_{m} & \\ m_{0} h_{0} & \rightleftharpoons & m_{1} h_{0} & \rightleftharpoons & m_{2} h_{0} & \rightleftharpoons & m_{3} h_{0} \\ & \beta_{m} & & 2 \beta_{m} & & 3 \beta_{m} & \end{array}$

In this description, each channel has 8 possible states and $\left[m_{i} h_{j}\right]$ is the number of channels within the channel population that are in state $m_{i} h_{j}$. An individual channel is open, when it is in the state $m_{3} h_{1}$, in all other states the channel is closed. Thus, the total sodium membrane conductance is given by:

$$
g_{\mathrm{Na}}=\gamma_{\mathrm{Na}}\left[m_{3} h_{1}\right]
$$

The population of voltage-gated potassium channel is modeled by the following kinetic scheme, which reflects the cooperative switching of four subunits required to open the channel:

$$
n_{0} \underset{\beta_{n}}{\longleftrightarrow} n_{1} \underset{2 \beta_{m}}{\longleftrightarrow} n_{2} \underset{3 \beta_{m}}{\longleftrightarrow} n_{3} \underset{4 \beta_{m}}{\longleftrightarrow} n_{4}
$$

Here, it is assumed that the channel has 5 possible states. The channel is open when it is in the state 
$n_{4}$. The total potassium conductance is then given by:

$$
g_{\mathrm{K}}=\gamma_{\mathrm{K}}\left[n_{4}\right]
$$

The rates $\alpha_{x}, \beta_{x}$ are the standard Hodgkin-Huxley transition rates (see Sec. 2.6).

In each simulation time step $\Delta t$, the number of channels that switch between states $A$ and $B$ with rate $r$ is determined by choosing a random number from a binomial distribution:

$$
\operatorname{Prob}\left(\Delta n_{A B}\right)=\left(\begin{array}{c}
n_{A} \\
\Delta n_{A B}
\end{array}\right) p^{\Delta n_{A B}}(1-p)^{\left(n_{A}-\Delta n_{A B}\right)},
$$

with $p=r \Delta t$. In Fig. 4.7, a simulation of a conductance-based model with different discrete numbers of sodium and a fixed number of potassium channels is shown. The single channel conductance is thereby scaled such that the total sodium conductance is kept constant. The same noisy current was injected into the three models. Despite the different number of channels, APs were emitted very reliably (Fig. 4.7A,C,E). With a decreasing number of channels, the phase plot trajectories became noisier (Fig. 4.7,B,D,F), but the steepness of the AP onsets did, however, not change. In a physiological realistic regime (Fig. 4.7C,D), the onset dynamics even differs only slightly from the limit of a large number of channels (Fig. 4.7A,B). The onset variability increases slightly but also does not reproduce the variability exhibited by APs in in vivo recordings. The scheme given by Eq. (4.29) is the most simple kinetic scheme which was proposed to model the activation and inactivation of the voltage-gated sodium channel. To test, if more complicated schemes would lead to a different activation dynamics, I also implemented schemes which include more dynamical steps for activation and inactivation (Patlak, 1991). With none of the schemes tested, the onset AP onset dynamics substantially changed.

These results imply that channel noise is not a suitable candidate to explain the in vivo recordings shown in Sec. 4.5. One has to keep in mind that here also the single channel conductance was scaled to keep the total conductance constant. With a constant single channel conductance, a membrane with 1200 channels would be unable to generate full APs.

\subsubsection{Where do APs initiate?}

So far, I did not comment on the spatial structure of a pyramidal neuron. In Fig. 4.8, a reconstructed pyramidal cell from layer V of the visual cortex is shown. Synaptic inputs arrive at different locations on the basal and distal dendrites and travel towards the soma. At which location are APs in cortical neurons initiated? Classically, it is assumed that the synaptic inputs which arrive at different positions of the dendritic arbor travel to the cortical soma where they are subsequently integrated and eventually lead to the generation of an AP.

Several recent studies, however, questioned this classical picture. Simultaneous recordings from the soma and the initial segment showed that APs which are generated in response to synaptic stimulation in Layer I are initiated beyond the initial segment in the axon (Häusser et al., 1995; Stuart et al., 1997). A second indication came from experiments, in which the sodium channel blocker TTX was applied locally either to the initial segment or to the axon beyond the initial segment (Colbert \& Johnston, 1996). Application in the axon raised the observed AP threshold by $7-10 \mathrm{mV}$. This was confirmed in experiments, in which the axon of a pyramidal neuron was cut (Colbert \& Pan, 2002). In these experiments, the authors showed that APs in neurons with cut axons initiate at considerably lower potentials. Furthermore, their data indicates (although not commented on by the authors) that 
A

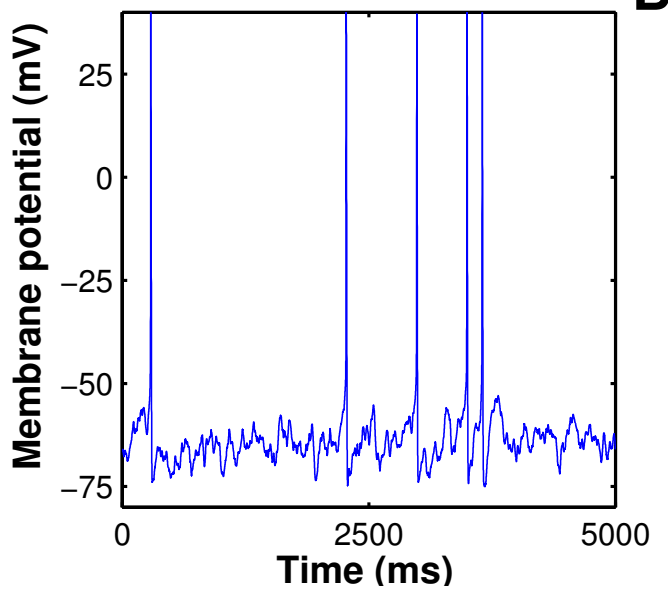

B

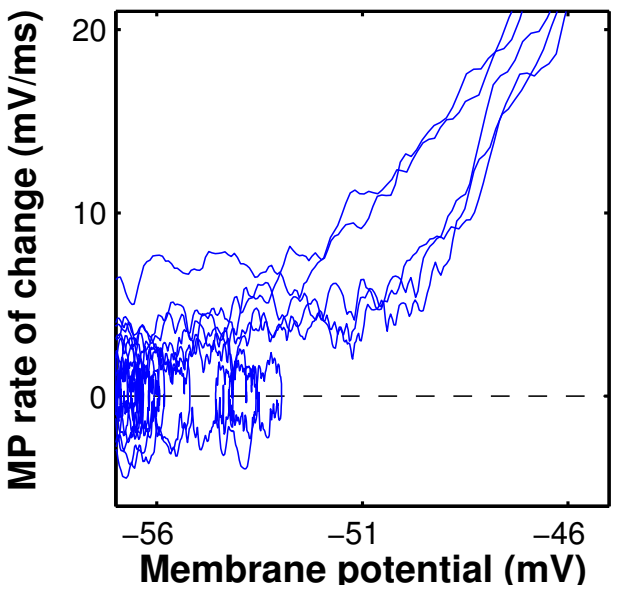

C

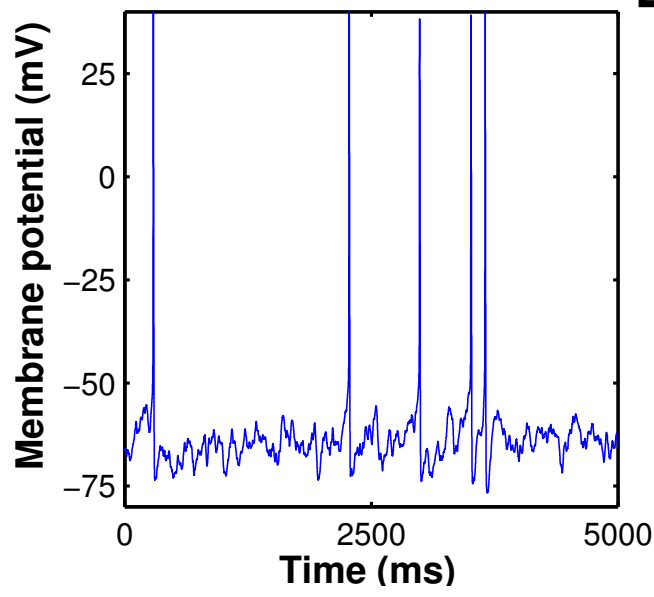

D

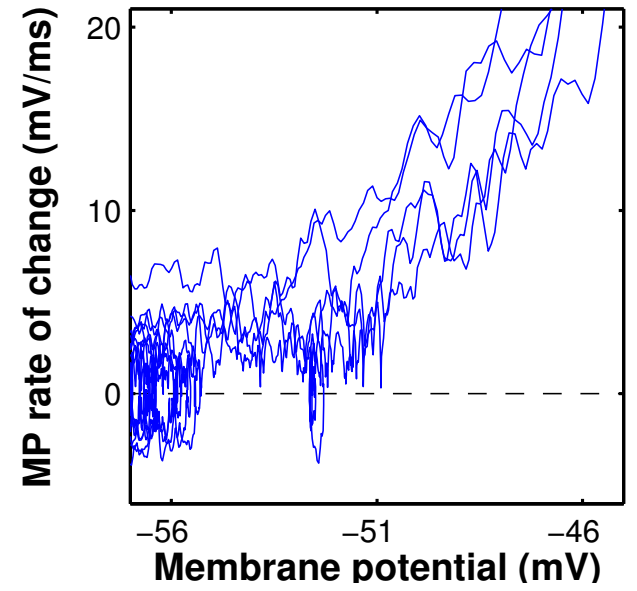

$\mathbf{E}$

$\mathbf{F}$

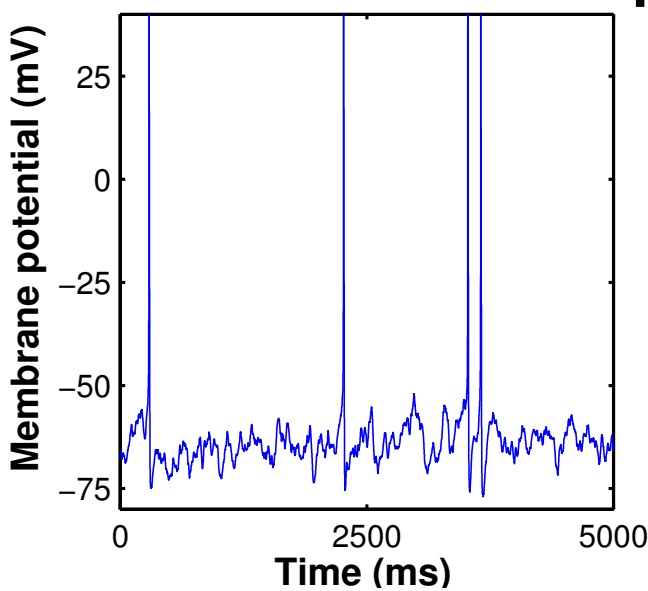

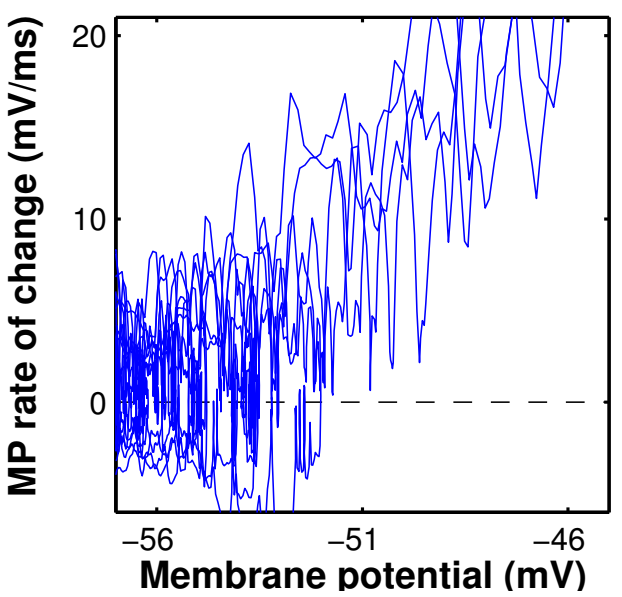

Figure 4.7: Impact of increasing channel noise on the onset dynamics of APs in a conductancebased neuron model. (A,C,E) Voltage trace of a model neuron with the same fluctuating synaptic background input and a different finite number of voltage-gated sodium (A: 120000, C: 12000, E: 1200) and potassium (3600) channels. (B,D,F): Corresponding phase plots. With a decreasing number of sodium channels the AP onset dynamics becomes noisier. The voltage-range over which APs initiate does, however, not change. 


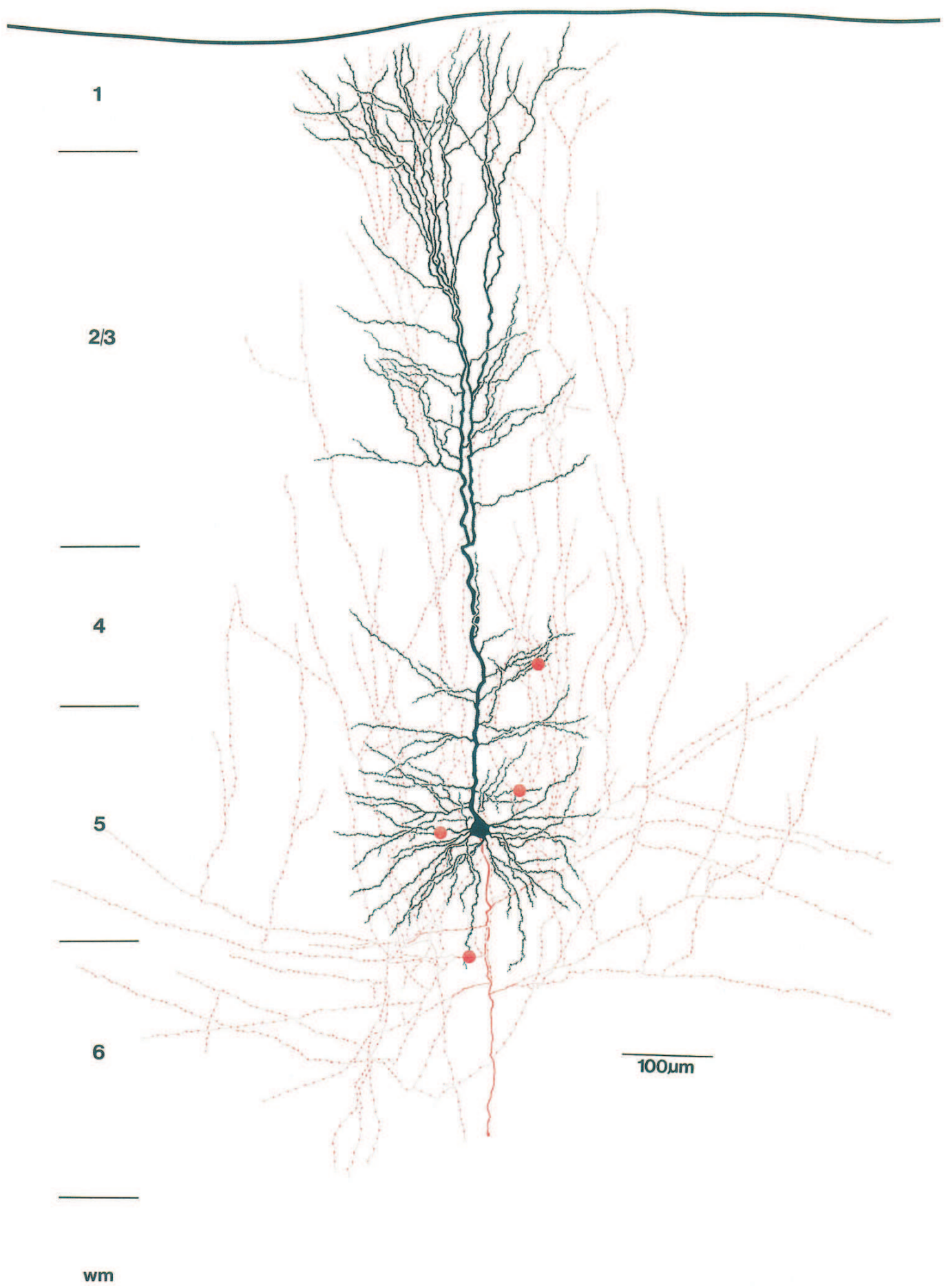

Figure 4.8: Where do APs initiate? Reconstruction of a Layer V cortical pyramidal neuron. For better visualization, the dendritic morphology of the neuron is drawn in black, whereas the axonal arborization is drawn in red. The red dots indicate autaptic contacts established onto basal and oblique dendrites by the axon. Note the extensive vertical axonal collaterals which go up to the cortical surface. Synaptic inputs arrive at all points of the dendritic arbor and give rise to depolarizations or hyperpolarizations at the soma. A central issue in the understanding of single neuron computation is to know at which location APs are initiated. Recent studies indicate that APs are initiated in the axon, rather than in the soma. (Adapted from Lübke et al. (1996)). 
APs which initiate somatically have a smooth onset behavior (as predicted by the Hodgkin-Huxley theory), whereas APs which initiate in the axon exhibit a rapid onset behavior. In recent, highly nontrivial experiments it was even demonstrated that APs in cerebellar Purkinje cells initiate at the first node of Ranvier (Clark et al., 2005).

These studies thus suggest that the special structure at the node of Ranvier may play a central role in the generation of the anomalous APs observed in cortical neurons. In Fig. 4.9, the highly structured organization, which is found at the node, is shown. A special sodium channel subtype, $N a_{V} 1.6$, is highly clustered in the central part of the node and spatially separated by an extracellular protein from other types of voltage-gated channels. The density of sodium channels at the node was estimated as $10^{3}$ channels $/ \mu \mathrm{m}^{2}$ (Chiu, 1980). Why did nature invent this highly specialized structure? In the next section, I present a mean field model that assumes that the dense packing of sodium channels at the node leads to a cooperative dynamics of their gating dynamics. In theoretical models, channel coupling was demonstrated to effectively change the slope of the activation curve of voltage-gated channels (Liu \& Dilger, 1993). The authors of this study modeled a one-dimensional chain lattice of voltage-gated channels, where each channel was assumed to interact with its neighboring channels. Using direct numerical simulations, they further showed that a two-dimensional lattice of voltagegated channels exhibits the same behavior. The analysis of the mean field model in the following section shows that there can even occur a discontinuity in the activation curve if the coupling between channels exceeds a critical value.

\subsubsection{Sodium channel coupling at the node of Ranvier}

To describe the coupling of sodium channels at the node, a two-state scheme will be used, in which I assume that each channel can be either in the closed $(\mathrm{C})$ or open $(\mathrm{O})$ state. Between those states, the channel can switch with rates $\alpha$ and $\beta$ :

$$
\begin{gathered}
\alpha(V) \\
C \stackrel{ }{\rightleftharpoons} O \\
\beta(V)
\end{gathered}
$$

The rates $\alpha$ and $\beta$ depend on the applied voltage $V$ across the membrane. The opening of the sodium channel is believed to incorporate at least three identical subunits. Here, I will discuss the simplest case, in which a single channel is modeled as a two-state system. For the point I would like to make, this restriction is not crucial, but the analysis of the model is considerably simpler.

I will assume that there are $N$ channels $\left\{c_{i}\right\}$ in the membrane, where each $c_{i}$ can take the values $\{0,1\}$, which corresponds to the closed (0) or open (1) state. A generic Hamiltonian which describes the total energy of the pair-wise coupled $N$-channel system is then given by:

$$
H=-\sum_{i=1}^{N} \varepsilon\left(c_{i}, V\right)-\frac{1}{2} \sum_{\{i, j\}} \varepsilon_{i j}^{(c)}\left(c_{i}, c_{j}\right),
$$

where $\varepsilon( \pm 1, V)$ is the single-channel energy in the closed or open state respectively, and $\varepsilon_{i j}(\cdot, \cdot)$ is the energy contribution due to coupling between pairs of channels. The single channel energy is in the simplest case given by $z\left(V-V_{0}\right) c_{i}$, where $V$ denotes the applied potential across the membrane, $V_{0}$ a voltage offset and $z$ the number of charges that need to be moved from the closed to the open state. 


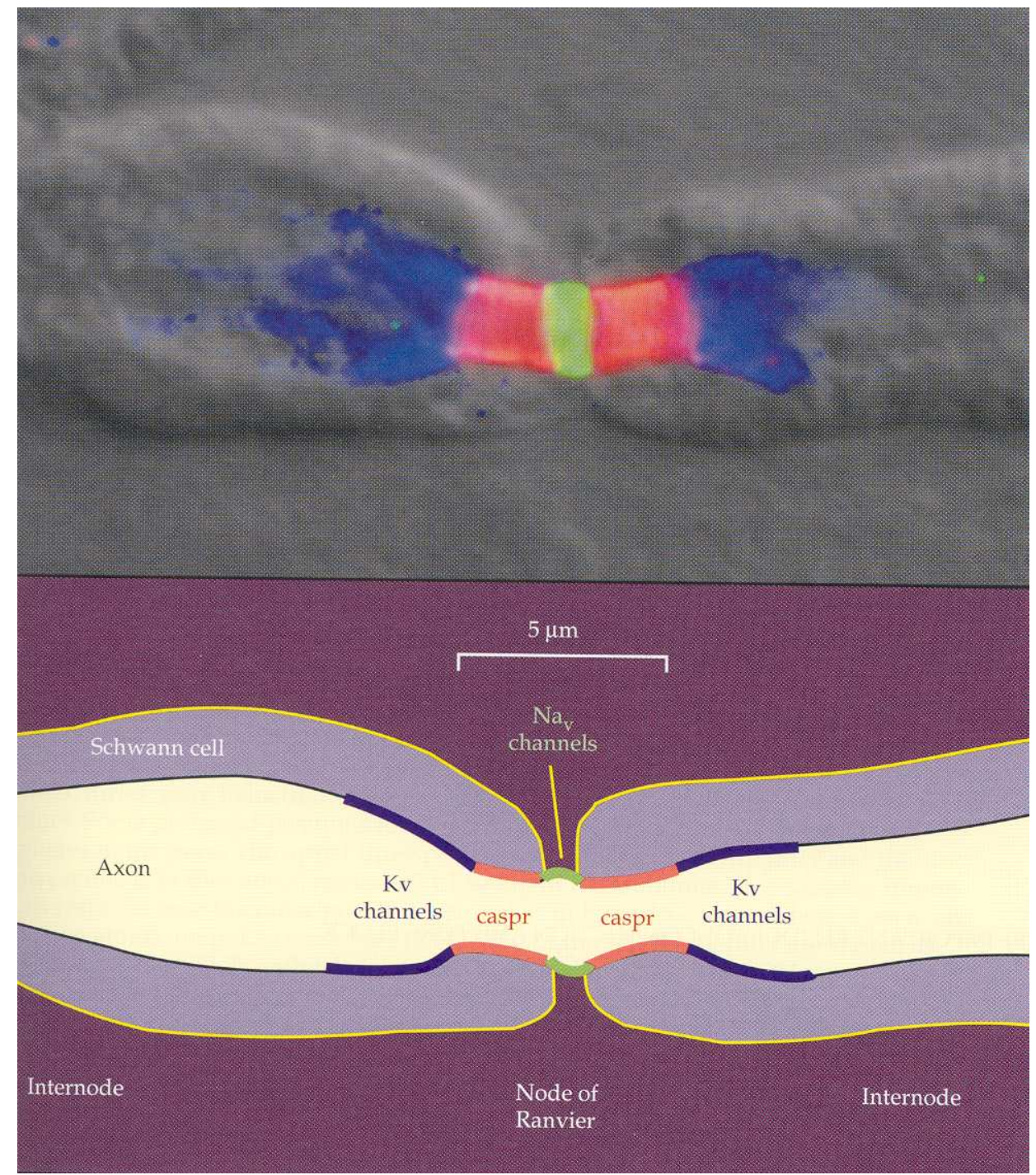

Figure 4.9: Clustering of ion channels in a myelinated nerve fiber. Flourescence micrograph (top) and schematic representation (bottom) of a short stretch of a rat myelinated nerve fiber including one node of Ranvier and the paranodal and juxtaparanodal regions on each side. A special type of voltage-gated sodium channels $\left(N a_{V} 1.6\right)$ is clustered at the node (green). The extracellular protein Caspr (red) lies on either side of the node in the paranodel region and marks the end of the myelin. Voltage gated potassium channels (blue) lie in the juxtaparanode, which is the initial region of the internodal axon. (Adapted from Rasband \& Shrager (2000)) 


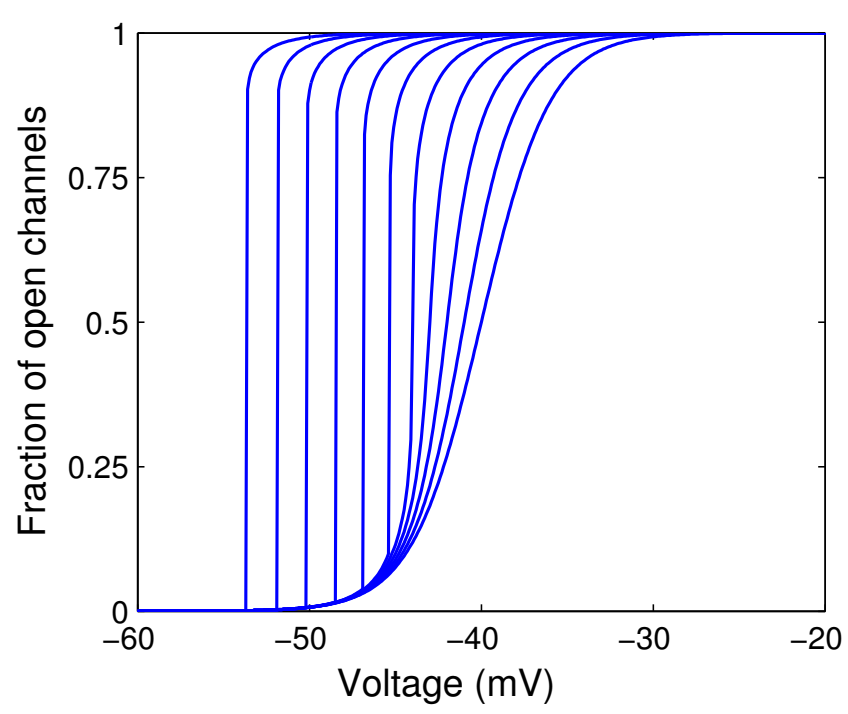

Figure 4.10: Solutions of the selfconsistent Eq. (4.38) for the opening probability of voltage-gated sodium channels. Increasing values of the coupling lead to an increasing sharpness of the activation curve. If a critical coupling strength is reached, the activation curve even exhibits a discontinuity. The voltage at which the discontinuity occurs depends on the coupling strength and can thus dynamically shift. Parameters: $\beta=2, z=1, V_{0}=-40 \mathrm{mV}$. The effective coupling strength $v \alpha$ takes the values $0,2, \ldots, 20$.

The coupling is modeled in the following mean-field form:

$$
\sum_{\{i, j\}} \varepsilon_{i j}^{(c)}\left(c_{i}, c_{j}\right)=\sum_{i} v \alpha\langle c\rangle c_{i}
$$

in which each channel is on average coupled to $v$ neighboring channels with coupling constant $\alpha$.

Without coupling $(\alpha=0)$, the probability to find channel $i$ in the open state is given by:

$$
P^{(v=0)}\left(c_{i}=1\right)=\frac{e^{\beta E(1)}}{e^{\beta E(0)}+e^{\beta E(1)}}=\frac{1}{1+e^{-\beta z\left(V-V_{0}\right)}}=\frac{1}{2}\left(1+\tanh \left(\frac{\beta z\left(V-V_{0}\right)}{2}\right)\right),
$$

which is the usual sigmoidal activation curve, similar to the activation curve which was introduced by Hodgkin and Huxley for the activation of the sodium channel subunit $m(t)$. From this, a self-consistent equation for the opening probability of the coupled system can be derived:

$$
[O] \equiv P\left(c_{i}=1\right)=\frac{1}{2}\left\{1+\tanh \left(\frac{\beta}{2}\left(z\left(V-V_{0}\right)+v \alpha[O]\right)\right)\right\}
$$

where I abbreviated the opening probability, i.e. the fraction of open channels with $[O]$. The presented approach is formally identical to the Weiss mean-field approximation of a multi-dimensional Isingsystem (Reichl, 1998).

In Fig. 4.10, the impact of an increased effective coupling strength $v \alpha$ is displayed. With increasing coupling, the mean field opening probability gets sharper. When the coupling constant $\alpha$ crosses a critical value, $\alpha_{\text {crit }}=4 /(v \beta)$, the activation curve even becomes discontinuous. As demonstrated before, the opening probability, i.e. the channel activation curve, directly reflects the onset dynamics at AP initiation. Thus, the induced coupling could account for the fast AP onset dynamics. The second characteristic feature of AP initiation is the large variability of onset potentials. The variable onset of the activation curve with varying coupling strength suggests a potential mechanism for this feature.

The possibility that ion channels exhibit cooperative dynamics has been investigated since it became 
possible to measure the dynamics of single channels using the patch-clamp technique. Although several initial studies indicated that gating of different channels are independent (Neher et al., 1978; Sigworth, 1980, 1981), there are now a number of studies which show that channels can coordinate their opening and closing states. These studies, which used a variety of different methods, found evidence for coupled gating of acetylcholine receptor channels (Yeramian et al., 1986), gap junction channels (Veenstra et al., 1994) and ryanodine receptor channels (Marx et al., 1998). One study found even direct evidence for a cooperative gating in voltage-gated sodium channels (Iwasa et al., 1986). Future experiments have to resolve if a coupling between voltage-gated channels is a suitable mechanism to explain the sharp onset of APs in cortical neurons.

\subsubsection{Non-canonical sodium channel activation}

A third mechanism assumes that the sharp onset of APs in cortical neurons is due to an intrinsic noncanonical activation of single channels. If this was true, the question arises, as to why the numerous experiments, which assessed the activation of voltage-gated sodium channels, did not find this noncanonical activation. To answer this question, it is instrumental to consider the fraction of sodium channels that is involved in the initial phase of an AP. A rough estimate shows that, at the most, only $10 \%$ of all available channels have to open to initiate an AP. All studies which assessed the activation curve of sodium channels expressed in pyramidal cells showed that the overall shape of this curve can be fitted quite well with a Boltzmann function. In no study, however, was the initial part of the activation curve measured with high precision. Published data (e.g. shown in Fig. 4.11) suggests that there may be strong deviations from a Boltzmann-fit exactly in the region which is relevant for AP initiation. A threshold-like start of the activation curve in this region could explain the measured onset dynamics in cortical neurons.

Indeed, a simple model, which assumes two consecutive steps for the activation of a single channel would be sufficient to implement this activation dynamics:

$$
\begin{aligned}
& \alpha_{1}(V) \quad \alpha_{2}(V) \\
& C_{1} \rightleftharpoons C_{2} \rightleftharpoons O \\
& \beta_{1}(V) \quad \beta_{2}(V)
\end{aligned}
$$

Assuming the standard rate functions for thermally activated state transitions,

$$
\alpha_{n}(V)=\exp \left(k_{n}\left(V-V_{n}\right)\right) ; \quad \beta_{n}(V)=\exp \left(-k_{n}\left(V-V_{n}\right)\right) \quad n \in\{1,2\},
$$

the fraction of open channels at a given voltage is given by:

$$
[O]=\frac{\exp \left(2\left(k_{1}+k_{2}\right) V\right)}{\exp \left(2\left(k_{1}+k_{2}\right) V\right)+\exp \left(2\left(k_{1} V+k_{2} V_{2}\right)\right)+\exp \left(2\left(k_{1} V_{1}+k_{2} V_{2}\right)\right)}
$$

If the activation of the initial step $C_{1} \rightleftharpoons C_{2}$ is sharp, the fraction of open channels will exhibit an initial kink, as shown in Fig. 4.12.

Moreover, the activation curve can be shifted by various mechanisms. Among them are changes in the external sodium concentration, external concentration of divalent ions (e.g. $\mathrm{Ca}^{2+}$ ) (Hille et al., 1975), and changes in $\mathrm{pH}$ (Woodhull, 1973) or phosphorylation (Cantrell \& Catterall, 2001), which might resolve the AP onset variability. 
A
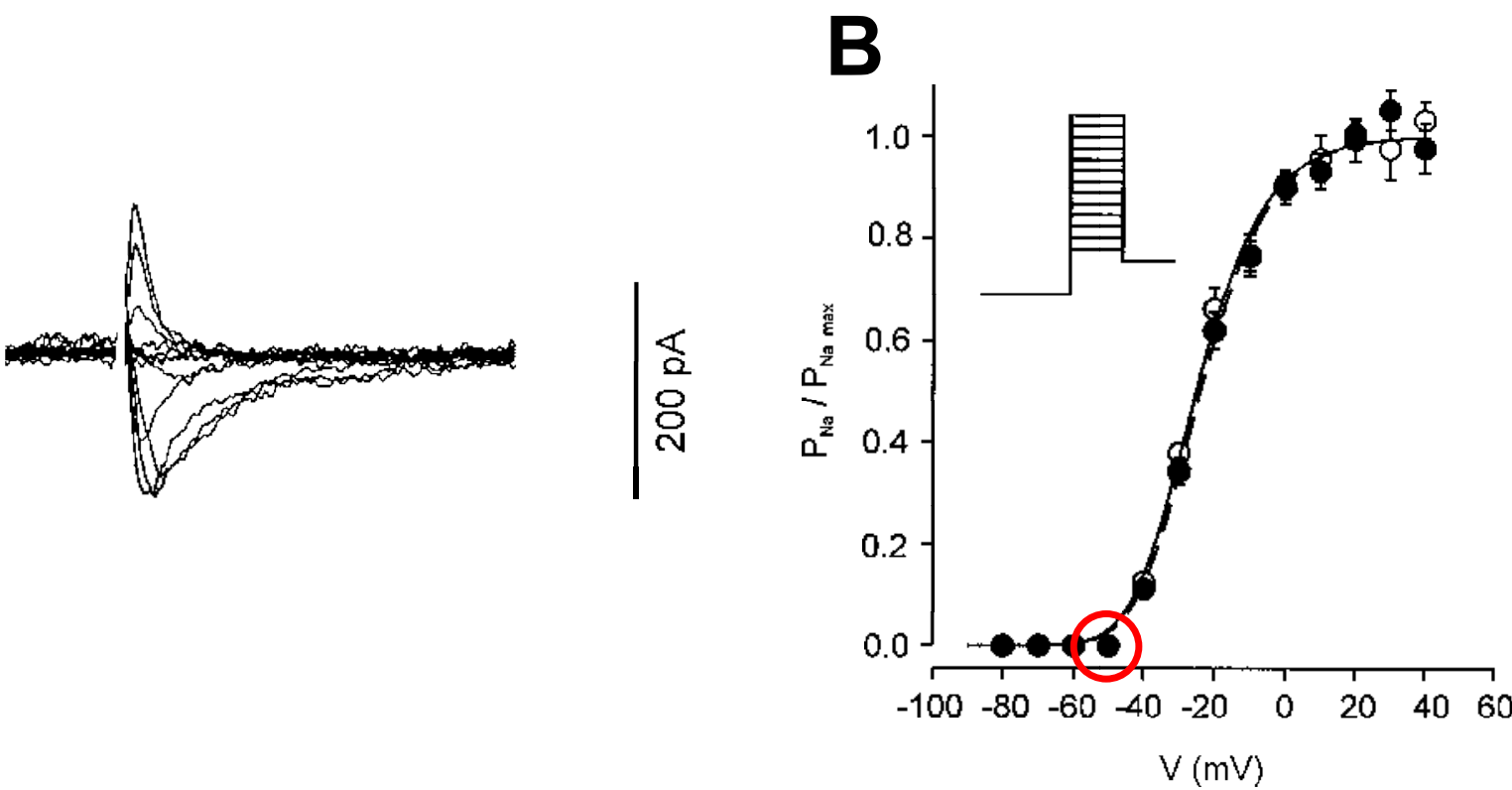

Figure 4.11: Voltage dependence of the activation of $\mathrm{Na}$ channels in patches of neuronal membrane. A: traces of $\mathrm{Na}$ currents recorded using a voltage-clamp protocol. The potential is first set to the holding potential $-90 \mathrm{mV}$, from where it is stepped to different command potentials ( $-80 \mathrm{mV}$ to $40 \mathrm{mV}$ in $10 \mathrm{mV}$ increments). B: Maximum current as a function of the command potential. Open circles and continuous curve: mean values and fit from 8 basket cell patches. Closed circles and dashed curve: mean values and fit from 13 pyramidal cell patches. Error bars are smaller than the symbol size. The curves are fits to Boltzmann functions raised to the third power. The initial part of the activation curve is not well fit by the Boltzmann function (red circle) and suggests a kink-like onset. (Adapted from Martina \& Jonas (1997))

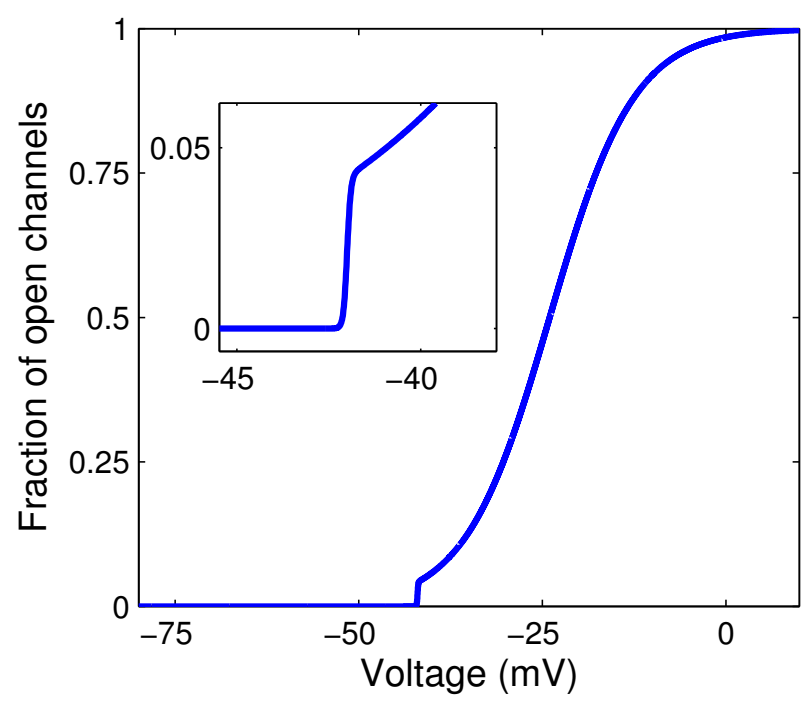

Figure 4.12: Activation curve resulting from a three-state kinetic model Eq. (4.39) of the voltage gated sodium channel. Assuming an initial step, which has a sharp activation, results in a kink-like onset behavior in the initial part of the activation curve. Parameters: $k_{1}=(0.1 \mathrm{mV})^{-1}, k_{2}=(11.5 \mathrm{mV})^{-1}$, $V_{1}=-42 \mathrm{mV}, V_{2}=-24 \mathrm{mV}$. 


\subsection{Summary}

In this chapter, I studied the AP initiation dynamics in in vivo and in vitro recordings of cortical neurons. Surprisingly, it turned out that AP initiation in these neurons exhibits an onset dynamics which is not compatible with standard Hodgkin-Huxley type models. APs are initiated much faster than predicted from standard sigmoidal shaped activation curves of voltage-gated sodium channels and at the same time in a large voltage range.

The anomalous AP initiation dynamics is not only present in in vivo recordings of cortical neurons from cat visual cortices, but is also observed in in vitro recordings of cortical neurons from rat, mouse and cat, and in neurons from the zebrafish olfactory bulb. This strongly suggests that the anomalous dynamics of cortical AP initiation is an intrinsic cellular property, found in neurons from different cortical and subcortical areas in many species. I discussed three candidate mechanisms to explain the anomalous dynamics of AP initiation. The first mechanism assumed that the discrete opening of voltage-gated channels in the membrane (channel stochasticity) leads to an abrupt opening of channels which is reflected in fast AP onsets. I showed, however, that with a realistic number of channels this affect can not account for the observed AP dynamics. I then presented two models, which can explain the fast onset dynamics. In the first model, I assumed that a coupling between voltage-gated channels at the densely packed node of Ranvier leads to a sharpening or even a discontinuity in the effective activation curve of the sodium channel population. In the second model I assumed that the opening of a channel subunit requires two subsequent kinetic steps. If the transition of the first step is sharp, the resulting activation curve exhibits a kink-like onset behavior at low potentials, compatible with patch-clamp recordings of voltage-gated sodium channels.

\subsection{Perspectives}

In the previous chapter, I showed that the AP onset dynamics crucially determines the speed with which ensembles of such neurons can respond to transient stimuli. This theoretical result indicates that the AP onset dynamics in cortical neurons is specifically optimized to encode rapidly varying stimuli.

I further showed that APs from cortical neurons do not only exhibit a fast onset dynamics but also a large variability in their onset potentials. Is this variability only a random dynamical epiphenomenon, unavoidable if APs initiate rapidly? In the next chapter I will introduce a phenomenological neuron model in which the variable onset potential is incorporated explicitly. It will turn out that the variable threshold serves as an effective adaptation mechanism which may play an important role in the ensemble coding of time-varying stimuli.

Still, many questions remain unanswered. What is the biophysical mechanism by which neurons surpass the Hodgkin-Huxley theory of AP initiation? What is the correct kinetic scheme for the activation of voltage-gated sodium channels? How do channels dynamically couple? Is there a sharpening of the AP initiation during development? What role does the spatial structure of pyramidal cells play in sharpening the AP onset dynamics? Future studies will have to answer these questions in order to completely characterize the dynamical and computational properties of cortical neurons. 


\section{Functional consequences of anomalous action potential initiation: An exactly solvable model}

\subsection{Introduction}

In the previous chapter, I presented evidence that AP initiation in cortical neurons is not explained by Hodgkin-Huxley type models of AP initiation. To understand the coding properties of neuronal populations, it is, however, crucial to have models at hand, which faithfully reproduce the dynamics exhibited by cortical neurons.

In chapter 3, a model was introduced, in which the impact of a fast AP onset dynamics was specifically assessed. Using this model, it was shown that there is a close connection between the speed with which a population of neurons can respond to time-dependent stimuli and the AP onset speed of single neurons in the population. One of the main results was that a fast AP onset dynamics is instrumental for representing rapid time-dependent input stimuli in the dynamic population firing response.

In this chapter, it is further analyzed, in which ways the AP initiation dynamics of cortical neurons is adapted for population coding. I will first show that in many neurons the variability of AP onset potentials in vivo, which was identified in the previous chapter as a key feature of cortical AP initiation, is highly correlated with the mean MP preceeding APs. I will then incorporate this "subthreshold adaptation mechanism" into a phenomenological neuron model. This model assumes a time-dependent threshold for AP initiation. The threshold integrates the subthreshold fluctuations of the MP with a time-constant which is directly deduced from the analysis of in vivo recordings. The dynamics of the MP is directly defined as a Gaussian stochastic process. Because of its simplicity, the model allows to give explicit expressions for many stationary and dynamical coding properties.

In particular, the model predicts that the subthreshold adaptation is instrumental in reducing the population response to slowly varying inputs, thus serving as a homeostatic adaptation mechanism, keeping the average output rate of the neuron fixed. Moreover, it turns out that the threshold adaptation in combination with a sharp AP onset turns the model into a high-pass filter. While slowly varying stimuli are suppressed, rapid stimuli are transmitted almost unattenuated.

Previous studies showed that at the onset of cortical APs in vivo, there is a correlation between the MP $V$ and its velocity $\dot{V}$ (Azouz \& Gray, 2000, 2003). The authors of these studies further proposed that this correlation serves as an effective threshold for the initiation of APs. In the Gaussian neuron model with threshold adaptation, it turns out that a correlation at AP onset between $V$ and $\dot{V}$ is a direct consequence of the adaptation mechanism.

To compare the predictions from the subthreshold adaptation mechanism with the predictions from a threshold in the $V-\dot{V}$ plane, I directly construct a model in which a threshold in the $V-\dot{V}$ plane is assumed for AP initiation. Surprisingly, its stationary firing rate strongly depends on the statistics of 


\section{Functional consequences of anomalous action potential initiation: An exactly solvable model}

the second derivative of the subthreshold MP fluctuations. In a realistic parameter regime, the model predicts firing rates which are more than one order of magnitude larger than predicted by the $V-\psi$ model. This indicates that it should be possible to tell the two mechanisms, subthreshold adaptation and a threshold in the $V-\dot{V}$ plane, apart experimentally.

To test the assumption that a threshold in the $V-\dot{V}$ plane is a good predictor of the occurrence of AP in real neurons, I thus analyze in vivo intracellular recordings of cortical neurons. It turns out that a threshold in the $V-\dot{V}$ plane is indeed a poor criterion for AP initiation. In most of the recordings analyzed, the predicted firing rate is by a factor of two larger than the actually recorded firing rate. In many cases the rates differ by more than one order of magnitude.

\subsection{Subthreshold adaptation}

In theoretical studies, the effects of threshold adaptation was so far only considered in models which assume spike-triggered adaptation currents (see e.g. (Tuckwell, 1978; Vasudevan \& Vittal, 1982; Wilbur \& Rinzel, 1983; Gerstner \& Kistler, 2002; Chacron et al., 2003; Benda \& Herz, 2003; Jolivet et al., 2004; Lindner \& Longtin, 2005)). Here, I will show evidence for a different adaptation mechanism, in which the threshold for AP initiation depends on the subthreshold fluctuations of the MP. I will first present experimental evidence for this adaptation mechanism, by showing that the potential at which APs initiate correlates with the mean MP preceeding it. I will then assess the consequences for dynamic population coding in a phenomenological neuron model. All in vivo intracellular recordings which were used were acquired and preprocessed as described in the previous chapter.

\subsection{Experimental evidence}

What is the relative influence of the mean MP preceeding an AP and the AP onset potential? To answer this question systematically, the correlation between the onset potentials $\left\{V_{\text {thres }}^{(i)}\right\}$ of APs in traces of in vivo intracellular recordings and the exponentially weighted mean MP preceeding them was calculated. The time constant $\tau$ of the exponential weighting was chosen such that the resulting correlation $r_{S A}$ was maximal:

$$
r_{S A}=\max _{\tau} \operatorname{Corr}\left(\left\{V_{\text {thres }}^{(i)}\right\},\left\{\int_{0}^{t_{i}} e^{-\left(t^{\prime}-t_{i}\right) / \tau} V\left(t^{\prime}\right) d t^{\prime}\right\}\right)
$$

In Fig. 5.1A, a sample scatter plot is shown, which demonstrates that for many traces, there is a strong correlation between the AP onset potentials and the mean MP preceeding them. The inset shows a histogram of the time-constants $\tau$ from different recordings, which vary from $10 \mathrm{~ms}$ to $100 \mathrm{~ms}$ with a mean value of about $30 \mathrm{~ms}$. In Fig. 5.1B a scatter plot is shown, graphing the $r_{S A}$ vs. the correlation coefficient between the AP onset potential and the ISI preceeding APs. Most points lie below the diagonal, indicating that the mean MP preceeding APs is a better predictor of the variability of the AP onset potentials than the ISI. 

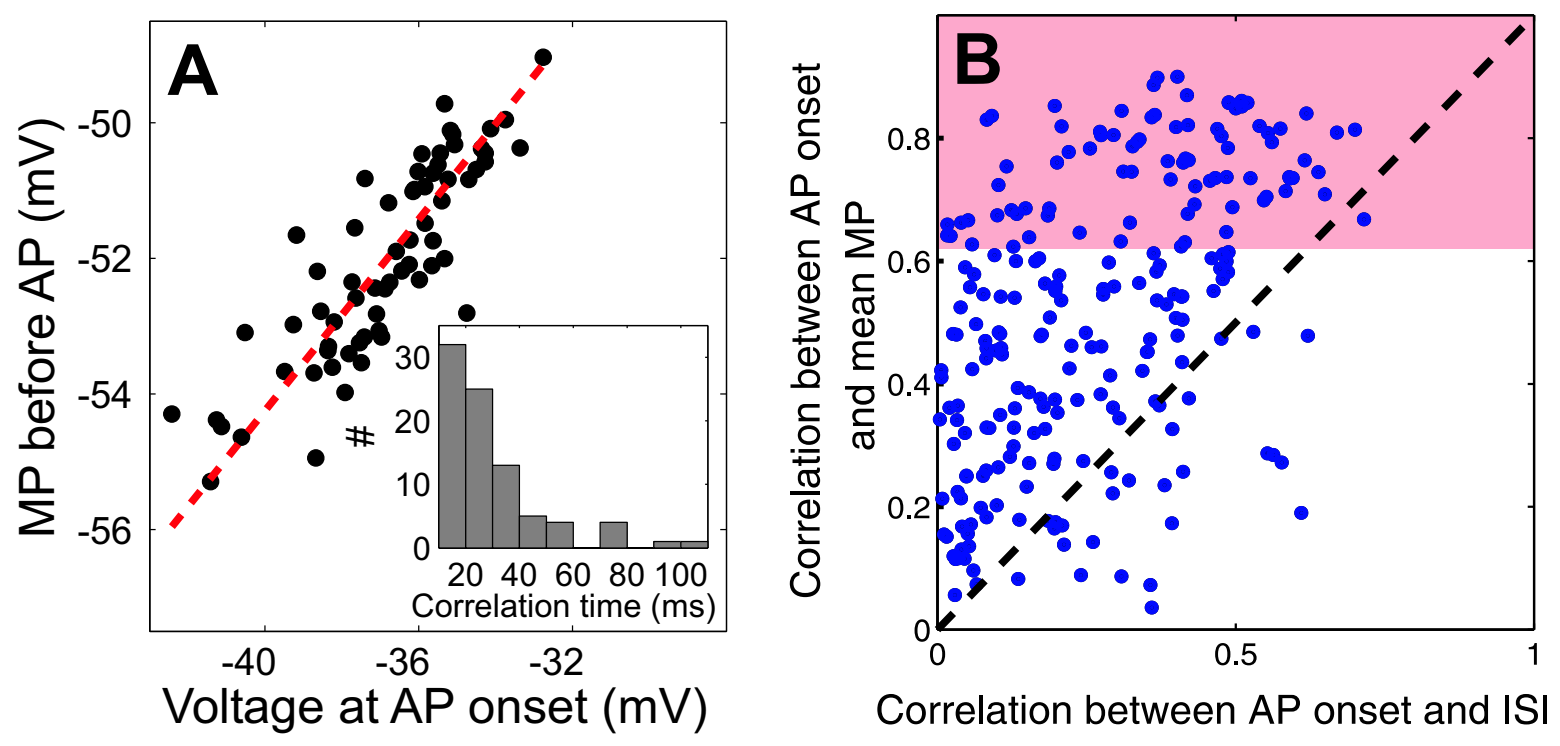

Figure 5.1: (A) AP onset potentials are highly correlated with the mean MP preceeding them. Representative scatter plot of AP onset potentials vs. the mean MP before the APs $(r=0.86, \tau=30 \mathrm{~ms})$. Inset: Histogram of the measured threshold time constants $(r>0.62, N=85)$. (B) Correlation between the MP before AP onset and the ISI before AP onset. Both covary, the MP correlation is typically larger. All data points in the red shaded region were taken for the analysis in $(A)$.

To determine the threshold time constants, the correlation between exponentially weighted prespike MPs with the AP onset potentials was maximized with respect to the time constant of the exponential filter. 


\section{Functional consequences of anomalous action potential initiation: An exactly solvable model}

\subsection{The Gaussian neuron model with subthreshold adaptation ( $V-\psi$ model)}

To understand the functional implications of the variable onset potential, I constructed a phenomenological neuron model, the Gaussian neuron model with subthreshold adaptation ( $V-\psi$ model). In this model, unlike in other phenomenological models, such as the LIF model, the dynamics of the MP is not defined by a differential equation, but directly as a Gaussian stochastic process $V(t)$ with given statistical properties. This process models the fluctuating MP, which is typical for cortical neurons in vivo (see Fig. 4.3). The theory of Gaussian random fields was first introduced into theoretical neuroscience in (Wolf \& Geisel, 1998), to describe the dynamics of pinwheels in orientation selectivity maps in the visual cortex.

The statistical properties of the MP fluctuations are completely characterized by the correlation function of the MP $C(\tau)$ and its mean value $\langle V(t)\rangle$, assuming that the fluctuations of the subthreshold MP are stationary. In the following analysis I will assume that the correlation function is of the form:

$$
C(\tau)=A\left(k_{2} \exp \left(-k_{1}|t|\right)-k_{1} \exp \left(-k_{2}|t|\right)\right)
$$

This choice ensures that the correlation function is twice differentiable at $t=0$, thus ensuring that the variance of the first temporal derivative of the MP exists. By choosing the parameters $A=\sqrt{2} \sigma_{V}^{3} / \sigma_{\dot{V}}$, $k_{1}=\sigma_{\dot{V}} /\left(\sqrt{2} \sigma_{V}\right)$ and $k_{2}=\sqrt{2} \sigma_{\dot{V}} / \sigma_{V}$, the variance of $u(t)$ and $\dot{u}(t)$ are given by:

$$
\begin{aligned}
& \left\langle(V(t)-\langle V(t)\rangle)^{2}\right\rangle=C(0)=\sigma_{V}^{2} \\
& \left\langle\dot{V}(t)^{2}\right\rangle=-\left.\frac{d^{2}}{d \tau^{2}} C(\tau)\right|_{\tau=0}=\sigma_{\dot{V}}^{2}
\end{aligned}
$$

where the dots denote first temporal derivatives.

So far, the model includes only passive membrane properties. AP generation is incorporated into the model by assuming a threshold potential $\psi(t)$, which explicitely depends on time. Each time the MP $V(t)$ crosses the time-dependent threshold from below, an AP is emitted. An important difference to the classical IF model is that there is no reset mechanism incorporated into the model. This may appear as a very crude simplification as the occurrence of an AP in a real neuron dramatically changes the MP time course and the intrinsic states of all voltage-gated channels. To reassure that we don't make a large error by introducing this approximation, it is expedient to first define the dynamical regime in which I will use the model in the following.

The correlation time of the MP $V(t)$ is given by:

$$
\tau_{c}=\sqrt{2} \ln \left(e+\sqrt{e^{2}-e}\right) \frac{\sigma_{V}}{\sigma_{\dot{V}}} \approx 2.2 \sigma_{V} / \sigma_{\dot{V}}
$$

For realistic parameters, $\sigma_{V}=3 \mathrm{mV}, \sigma_{\dot{V}}=2 \mathrm{mV} / \mathrm{ms}$, the correlation time is hence given by $\tau_{c} \approx$ $3.4 \mathrm{~ms}$. The average firing rate on the other hand does hardly ever exceed $10 \mathrm{~Hz}$, the spontaneous firing rate of a cortical neuron in vivo is, typically, even much lower $<5 \mathrm{~Hz}$. The mean ISI is then at least $100 \mathrm{~ms}$. This means that if the firing rate is reasonably small, the MP will not "remember" if it was reset after an AP was generated. The big advantage of the applied approximation is that many stationary and dynamic response properties can be calculated without any approximations. This is not 


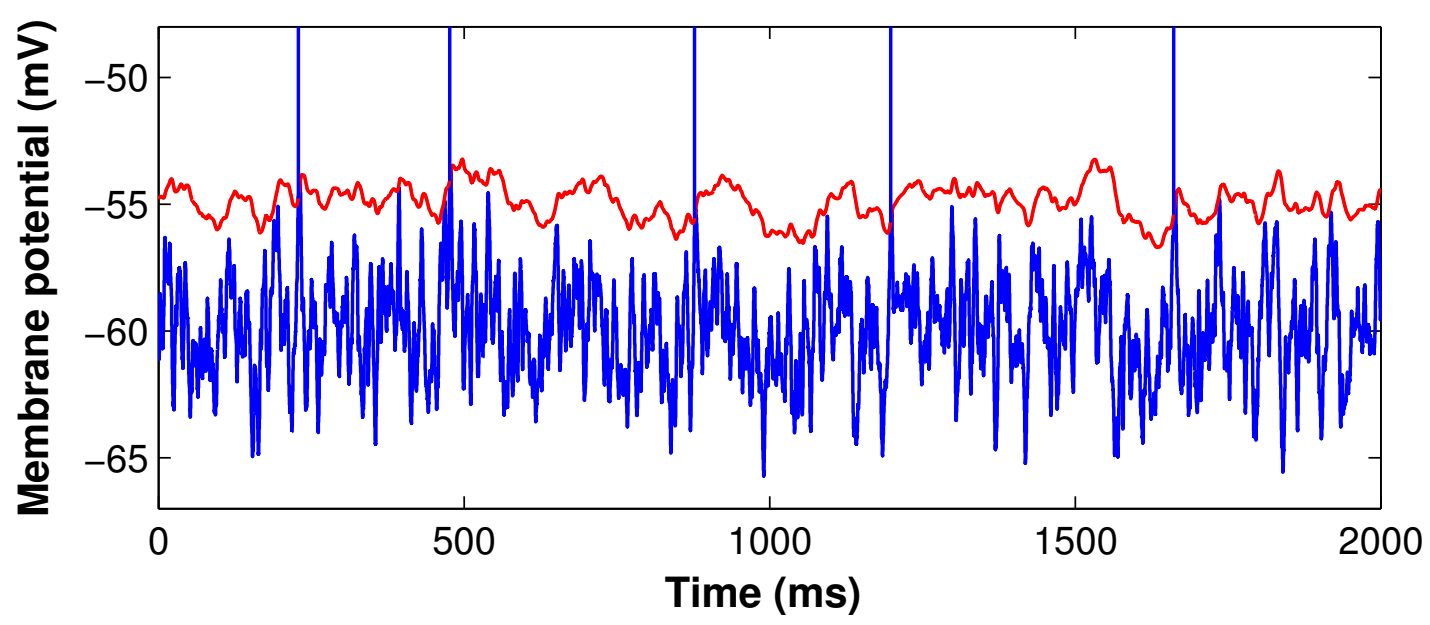

Figure 5.2: Sample trajectory of the $V-\psi$ model. The subthreshold MP trajectory is shown in blue, the time-dependent threshold in red. Whenever the MP reaches the threshold, an AP is generated (indicated as blue vertical lines). To simulate the Gaussian process, a two-dimensional OrnsteinUhlenbeck process was used. Parameters: $\sigma_{V}=2 \mathrm{mV}, \sigma_{\dot{V}}=1 \mathrm{mV} / \mathrm{ms}, \psi_{0}=5 \mathrm{mV}, \tau_{\psi}=30 \mathrm{~ms}$.

possible in standard IF models with realistic input currents, as I discussed in Chap. 3.

The dynamics of the threshold is modeled by a first order kinetics, driven by the MP $V(t)$ with a time constant $\tau_{\psi}$ :

$$
\tau_{\psi} \dot{\psi}(t)=\left(\psi_{0}-\psi(t)\right)+c\left(V(t)-V_{0}\right),
$$

where $\psi_{0}$ is the mean threshold voltage and $c$ the coupling between the threshold and the MP. In Fig. 5.2, a sample realization of a model trajectory is displayed. Whenever the MP crosses the timedependent threshold from below, an AP is emitted.

As I am interested in the population coding of the $V-\psi$ model, in the following population averaged quantities will be considered. As in the assessment of the population coding properties of the generalized $\theta$-neuron (cf. chapter 5), it will be assumed that the MP fluctuations in each neuron are independent. Before discussing the impact of the time varying threshold on the dynamic response properties, it will be shown, how stationary response properties are calculated in this model.

The average number of APs in a population of neurons in the time interval $(t, t+\Delta t]$ is given by:

$$
\begin{aligned}
\langle N\rangle & =\left\langle\int_{t}^{t+\Delta t} d t^{\prime} \delta\left(V\left(t^{\prime}\right)-\psi\left(t^{\prime}\right)\left|\dot{V}\left(t^{\prime}\right)-\dot{\psi}(t)\right| \Theta\left(\dot{V}\left(t^{\prime}\right)\right)\right\rangle\right. \\
& =\int_{t}^{t+d t} d t^{\prime}\left\langle\delta\left(V\left(t^{\prime}\right)-\psi\left(t^{\prime}\right)\left|\dot{V}\left(t^{\prime}\right)-\dot{\psi}(t)\right| \Theta\left(\dot{V}\left(t^{\prime}\right)\right)\right\rangle\right. \\
& =\int_{t}^{t+\Delta t} d t^{\prime} v\left(t^{\prime}\right),
\end{aligned}
$$

where the angular brackets $\langle\cdot\rangle$ denote population averages. In the last equation, I introduced the time-dependent firing rate $v(t)$, which is defined as:

$$
v(t)=\int_{-\infty}^{\infty} d V(t) d \dot{V}(t) d \psi(t) \delta(V(t)-\psi(t))|\dot{V}(t)-\dot{\psi}(t)| \Theta(\dot{V}(t)) P(V(t), \dot{V}(t), \psi(t))
$$




\section{Functional consequences of anomalous action potential initiation: An exactly solvable model}

Here $\delta(\cdot)$ denotes the Dirac distribution and $\Theta(\cdot)$ is the Heaviside function. The joint pdf of $V(t), \dot{V}(t)$ and $\psi(t), P(V(t), \dot{V}(t), \psi(t))$ is given by:

$$
P(V(t), \dot{V}(t), \psi(t))=\left((2 \pi)^{3 / 2} \sqrt{\operatorname{det} \mathbf{C}}\right)^{-1} \exp \left\{-\frac{1}{2}\left(\begin{array}{c}
V(t)-V_{0} \\
\dot{V}(t) \\
\psi(t)-\psi_{0}
\end{array}\right) \mathbf{C}^{-1}\left(\begin{array}{c}
V(t)-V_{0} \\
\dot{V}(t) \\
\psi(t)-\psi_{0}
\end{array}\right)^{T}\right\}
$$

Note that the pdf does not depend on the velocity of the threshold $\dot{\psi}(t)$, as $\dot{\psi}(t)$ is determined by $V(t)$ and $\psi(t)$ (Eq. (5.6)).

For the case of a constant threshold, $\psi=\psi_{0}$, the firing rate reduces to:

$v_{F T}=\left(2 \pi \sigma_{v} \sigma_{\dot{V}}\right)^{-1} \int_{-\infty}^{\infty} d V(t) d \dot{V}(t) \delta\left(V(t)-\psi_{0}\right)|\dot{V}(t)| \Theta(\dot{V}(t)) \exp \left\{-\frac{1}{2}\left(\frac{\left(V(t)-V_{0}\right)^{2}}{\sigma_{V}^{2}}+\frac{\dot{V}(t)^{2}}{\sigma_{\dot{V}}^{2}}\right)\right\}$,

since for a stationary process $V(t)$, the covariance $\langle V(t) \dot{V}(t)\rangle$ is zero. The integral for the firing rate can be solved explicitly in this case, yielding:

$$
v_{F T}=(\sqrt{8 \pi})^{-1} \exp \left\{-\frac{\psi_{0}^{2}}{2 \sigma_{V}^{2}}\right\} \frac{\sigma_{\dot{V}}}{\sigma_{V}}
$$

With threshold adaptation, the correlation matrix $\mathbf{C}$ also includes the covariances between the threshold $\psi(t)$, the MP $V(t)$ and its velocity $\dot{V}(t)$ :

$$
\begin{aligned}
\mathbf{C} & =\left(\begin{array}{ccc}
\left\langle V(t)^{2}\right\rangle-\langle V(t)\rangle^{2} & \langle V(t) \dot{V}(t)\rangle & \langle V(t) \psi(t)\rangle-\langle V(t)\rangle\langle\psi(t)\rangle \\
\langle V(t) \dot{V}(t)\rangle & \left\langle\dot{V}(t)^{2}\right\rangle & \langle\dot{V}(t) \psi(t)\rangle \\
\langle V(t) \psi(t)\rangle-\langle V(t)\rangle\langle\psi(t)\rangle & \langle\dot{V}(t) \psi(t)\rangle & \left\langle\psi(t)^{2}\right\rangle-\langle\psi(t)\rangle^{2}
\end{array}\right) \\
= & \left(\begin{array}{ccc}
\sigma_{V}^{2} & \frac{c \sigma_{V}^{3}\left(2 \sigma_{V}+3 \sqrt{2} \sigma_{\dot{V}} \tau_{\psi}\right)}{\left(\sigma_{V}+\sqrt{2} \sigma_{V} \tau_{\psi}\right)\left(2 \sigma_{V}+\sqrt{2} \sigma_{\dot{V}} \tau_{\psi}\right)} \\
0 & \sigma_{\dot{V}}^{2} & -\frac{2 c \sigma_{\dot{V}}^{2} \sigma_{V}^{2} \tau_{\psi}}{2 \sigma_{v}^{2}+3 \sqrt{2} \sigma_{V} \tau_{V} \tau_{\psi}+2 \sigma_{\dot{V}}^{2} \tau_{\psi}^{2}} \\
\frac{c \sigma_{V}^{3}\left(2 \sigma_{V}+3 \sqrt{2} \sigma_{\dot{V}} \tau_{\psi}\right)}{\left(\sigma_{V}+\sqrt{2} \sigma_{V} \tau_{\psi}\right)\left(2 \sigma_{V}+\sqrt{2} \sigma_{\dot{V}} \tau_{\psi}\right)} & -\frac{2 c \sigma_{\dot{V}}^{2} \sigma_{V}^{2} \tau_{\psi}}{2 \sigma_{v}^{2}+3 \sqrt{2} \sigma_{\dot{V}} \sigma_{V} \tau_{\psi}+2 \sigma_{\dot{V}}^{2} \tau_{\psi}^{2}} & \frac{c^{2} \sigma_{V}^{3}\left(2 \sigma_{V}+3 \sqrt{2} \sigma_{\dot{V}} \tau_{\psi}\right)}{\left(\sigma_{V}+\sqrt{2} \sigma_{\dot{V}} \tau_{\psi}\right)\left(2 \sigma_{V}+\sqrt{2} \sigma_{\dot{V}} \tau_{\psi}\right)}
\end{array}\right)
\end{aligned}
$$

In this case, the rate is given by the double integral:

$$
\begin{gathered}
v=\tau_{\psi}^{-1} \int_{-\infty}^{\infty} d V(t) \int_{-\infty}^{\infty} d \dot{V}(t)\left|\tau_{\psi} \dot{V}(t)-\left(\left(\psi_{0}-V(t)\right)+c\left(V(t)-V_{0}\right)\right)\right| \\
\Theta(\dot{V}(t)) P(V(t), \dot{V}(t)),
\end{gathered}
$$

in which the threshold dynamics is included implicitly in the pdf $P(\cdot, \cdot, \cdot)$ and in the modulus in the integral kernel. In Fig. 5.3, the stationary firing rate as a function of $\tau_{\psi}$ for various values of $c$ is displayed. For values of $c$ close to 1 , the rate is almost zero for $\tau_{\psi} \rightarrow 0$ and increases to the value $v_{F T}$ predicted by Eq. (5.13). For decreasing values of $c$, the rate increases but always stays below $v_{F T}$.

Intuitively, for small values of $\tau_{\psi}$ the threshold follows the MP, thus suppressing all threshold crossings. For $\tau_{\psi} \rightarrow \infty$, the threshold becomes constant and its variance $\sigma_{\psi}^{2}$ goes to zero as $3 c^{2} \sigma_{V}^{3} /\left(\sqrt{2} \sigma_{\dot{V}} \tau_{\psi}\right)$. Thus, in this limit the model approaches the stationary rate of the Gaussian neuron with a fixed thresh- 


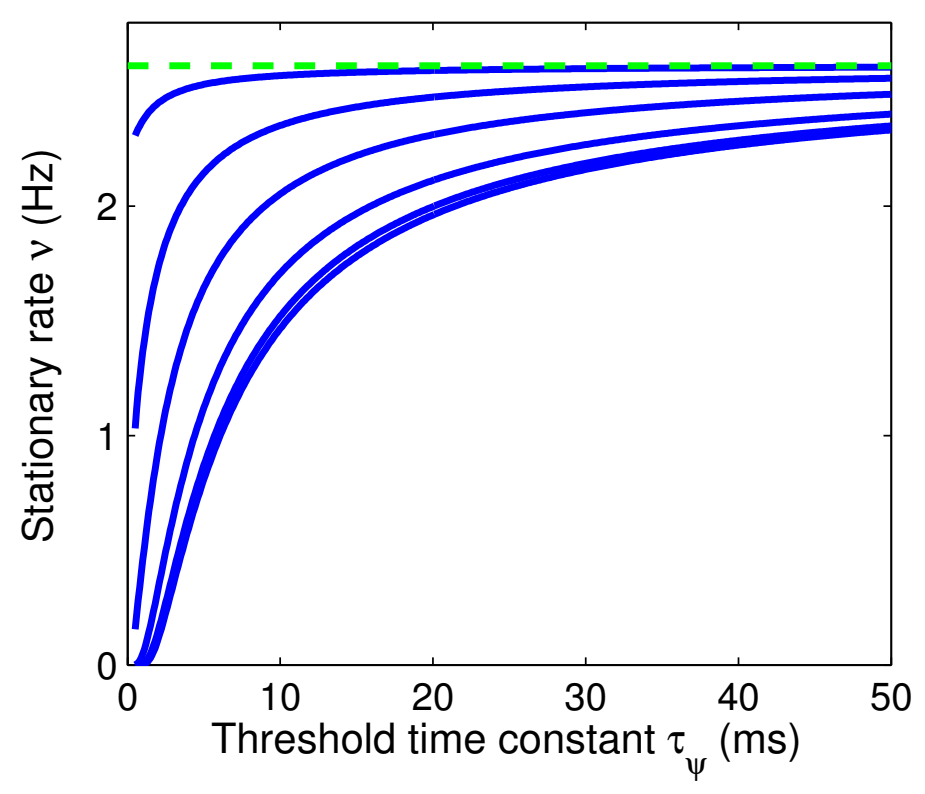

Figure 5.3: Stationary rate of the $V-\psi$ model. Solid lines: Stationary rate $v$ calculated via Eq. (5.16) for different values of the threshold coupling constant $c(0.01,0.1,0.25,0.5,0.75,1.0)$. Dashed line: Rate in the limit $c \rightarrow 0$.

The rate increases for larger values of $\tau_{\psi}$, as the threshold integrates over an increasingly long subthreshold MP trajectory. When the coupling between the threshold and then MP is increased, the rate decreases. Parameters: $\sigma_{V}=2 \mathrm{mV}, \sigma_{V}=3 \mathrm{mV} / \mathrm{ms}, \psi_{0}=6 \mathrm{mV}$. 


\section{Functional consequences of anomalous action potential initiation: An exactly solvable model}

old.

\subsection{Spike-triggered average membrane potential}

An important quantity used to characterize the MP trajectories which lead to the generation of APs is the spike-triggered average MP. Within the formalism of the $V-\psi$ model this quantity can be calculated in a straightforward way:

$$
\begin{aligned}
& \operatorname{STA}(\tau)= v^{-1}\left\langle\left(V(t-\tau)-V_{0}\right) \delta(V(t)-\psi(t)) \Theta(\dot{V})|\dot{V}(t)-\dot{\psi}(t)|\right\rangle \\
&= v^{-1} \int_{-\infty}^{\infty} d V(t) d V(t-\tau) d \dot{V}(t) d \psi(t)\left(V(t-\tau)-V_{0}\right) \delta(V(t)-\psi(t)|\dot{V}(t)-\dot{\psi}(t)| \Theta(\dot{V}(t)) \\
& P_{\mathrm{STA}}(V(t), V(t-\tau), \dot{V}(t), \psi(t))
\end{aligned}
$$

This integral now involves the joint pdf of $V(t-\tau), V(t), \dot{V}(t)$ and $\psi(t)$. The covariances of the latter three degrees of freedom were already needed in the previous section to calculate the rate. The covariances with $V(t-\tau)$ are given by:

$$
\begin{aligned}
&\langle V(t) V(t-\tau)\rangle-\langle V(t)\rangle\langle V(t-\tau)\rangle=C(\tau) \\
&\langle\dot{V}(t) V(t-\tau)\rangle-\langle\dot{V}(t)\rangle\langle V(t-\tau)\rangle=\left.\left(\frac{d}{d t} C(t-s)\right)\right|_{s \rightarrow t-\tau} \\
&=\sqrt{2} \sigma_{V} \sigma_{\dot{V}}\left(e^{-\frac{\sqrt{2} \tau \sigma_{\dot{V}}}{\sigma_{V}}}+e^{-\frac{\tau \sigma_{\dot{V}}}{\sqrt{2} \sigma_{V}}}\right) \\
&\langle\psi(t) V(t-\tau)\rangle-\langle\psi(t)\rangle\langle V(t-\tau)\rangle=c \tau^{-1} \int_{-\infty}^{0} d t C(t+\tau) e^{t / \tau} \\
&=\frac{c \sigma_{V}^{3} e^{-\tau\left(\frac{\sqrt{2} \sigma_{\dot{V}}}{\sigma_{V}}+\tau_{\psi}^{-1}\right)}}{2 \sigma_{V}^{4}-5 \sigma_{\dot{V}}^{2} \sigma_{V} \tau_{\psi}^{2}+2 \sigma_{\dot{V}}^{4} \tau_{\psi}^{4}} \\
&\left(6 \sqrt{2} e^{\frac{\sqrt{2} \sigma_{\dot{V}} \tau}{\sigma_{V}}} \sigma_{\dot{V}}^{3} \tau_{\psi}^{3}+e^{\tau / \tau_{\psi}}\left(2 e^{\frac{\tau \sigma_{\dot{V}}}{\sqrt{2} \sigma_{V}}}\left(2 \sigma_{V}+\sqrt{2} \sigma_{\dot{V}} \tau_{\psi}\right)\left(\sigma_{v}^{2}-2 \sigma_{\dot{V}}^{2} \tau_{\psi}^{2}\right)\right.\right. \\
&\left.\left.-\left(\sigma_{V}+\sqrt{2} \sigma_{\dot{V}} \tau_{\psi}\right)\left(2 \sigma_{V}^{2}-\sigma_{\dot{V}}^{2} \tau_{\psi}^{2}\right)\right)\right) \\
&\langle V(t-\tau) V(t-\tau)\rangle-\langle V(t-\tau)\rangle\langle V(t-\tau)\rangle=\sigma_{V}^{2}
\end{aligned}
$$

The pdf $P_{\mathrm{STA}}(V(t), V(t-\tau), \dot{V}(t), \psi(t))$ is then given by:

$$
\begin{aligned}
& P_{\mathrm{STA}}(V(t), V(t-\tau), \dot{V}(t), \psi(t))=\left(2 \pi \sqrt{\operatorname{det} \mathbf{C}_{\mathrm{STA}}}\right)^{-1} \\
& \exp \left\{-\frac{1}{2}\left(\begin{array}{c}
V(t)-V_{0} \\
V(t-\tau)-V_{0} \\
\dot{V}(t) \\
\psi(t)-\psi_{0}
\end{array}\right) \mathbf{C}_{\mathrm{STA}}^{-1}\left(\begin{array}{c}
V(t)-V_{0} \\
V(t-\tau)-V_{0} \\
\dot{V}(t) \\
\psi(t)-\psi_{0}
\end{array}\right)\right\},
\end{aligned}
$$


where the correlation matrix $\mathbf{C}_{\mathrm{STA}}$ extends the matrix $\mathbf{C}$ with the correlators of $V(t-\tau)$. The STA is then given by the following three-fold integral:

$$
\begin{aligned}
\operatorname{STA}(\tau)= & v^{-1} \int_{-\infty}^{\infty} d V(t-\tau) \int_{0}^{\infty} d \dot{V}(t) \int_{-\infty}^{\infty} d \psi(t) \\
& \left(V(t-\tau)-V_{0}\right)|\dot{V}(t)-\dot{\psi}(t)| P_{\mathrm{STA}}(\psi(t), V(t-\tau), \dot{V}(t), \psi(t)),
\end{aligned}
$$

with $\dot{\psi}(t)$ defined by Eq. (5.6). In Fig. 5.4, the STAs for different values of $c$ and threshold time constants $\tau_{\psi}$ are shown. Although the dynamics of the MP and of the threshold contains no oscillatory components, the STA exhibits an undershoot behavior. This time-course of the STA is, however, intuitively interpreted: To elicit an AP it is instrumental to first depolarize the MP over the time $\tau_{\psi}$, such that the threshold moves down. A rapid depolarization will then elicit an AP. As the pdf of the MP is Gaussian, this time course is more probable than a direct depolarization starting from the mean MP.

In in vitro recordings of pyramidal cells in which fluctuating currents were injected, this form of the STA was indeed observed (Mainen \& Sejnowski, 1995). It was previously attributed to a oscillatory subthreshold behavior in these neurons. Cortical pyramidal neurons were, however, shown to exhibit Type-I excitable behavior. In this case, an oscillatory subthreshold component would not be expected (Tateno et al., 2004). In the $V-\psi$ model, the undershoot of the STA is a direct consequence of the subthreshold adaptation, which was also likely to be present in the recordings mentioned.

Again, the $V-\psi$ model is the only proposed phenomenological neuron model which allows to calculate the STA analytically. In conventional IF models, one has to resort to numerical simulations, which are typically time-consuming and give only approximate results.

\subsection{Dynamic response theory}

In this section I will assess the dynamic response properties of the $V-\psi$ model. It will turn out that, contrary to the standard LIF model, it is even possible to calculate the response properties exactly, and to give explicit expressions in the linear response regime.

\subsubsection{Response function for inputs with large amplitudes}

To assess the time-dependent response properties of the $V-\psi$ model, a low-pass filtered signal $f(t)$ is added to the MP $V(t)$. The signal $f(t)$ is given by:

$$
\tau_{M} \dot{f}(t)=-f(t)+\operatorname{Signal}(t),
$$

where $\tau_{M}$ denotes the relaxation time of the membrane.

For the following analysis, I assume that the time-dependent input signal is given by a cosine with amplitude $A$ and frequency $\omega$. The function $f(t)$ is thus given by:

$$
f(t)=\frac{A \tau_{M}^{-2}}{\tau_{M}^{-2}+\omega^{2}}\left(\cos (\omega t)+\tau_{M} \omega \sin (\omega t)\right)
$$



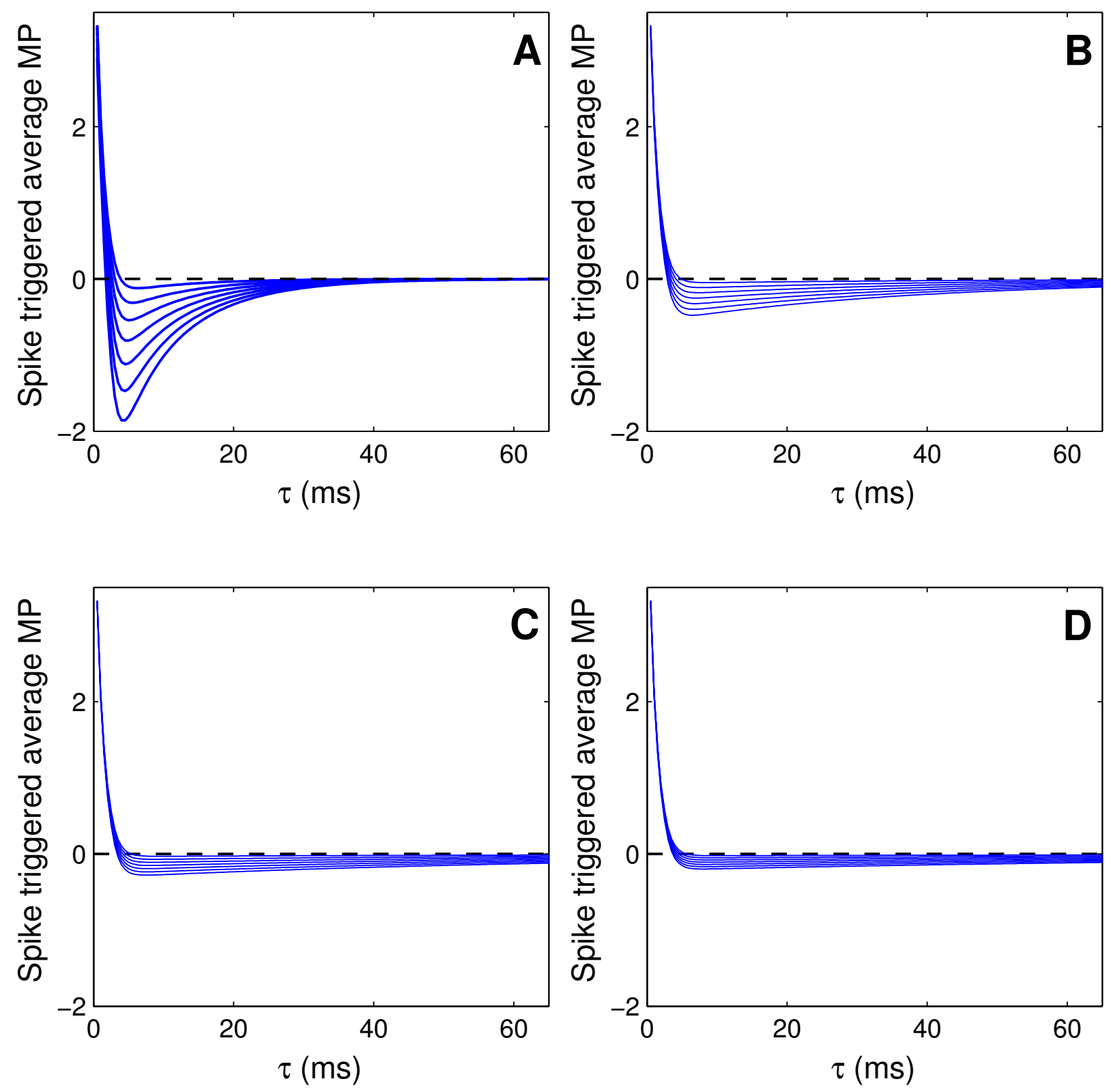

Figure 5.4: Spike-triggered average MP of the $V-\psi$ model. (A-D): Different threshold time constants $\tau_{\psi}(A: 10 \mathrm{~ms}, B: 40 \mathrm{~ms}, C: 70 \mathrm{~ms}, \mathrm{D}: 100 \mathrm{~ms})$. In each panel, the STA is shown for different threshold coupling constants $c$. For small values of $\tau_{\psi}$ and a strong coupling, the STA exhibits a pronounced undershoot, which decreases for increasing values of $\tau_{\psi}$ and decreasing coupling constants $c(0.1$, $0.25,0.4,0.55,0.7,0.85,1.0)$. Parameters: $\sigma_{V}=2 \mathrm{mV}, \sigma_{\dot{V}}=3 \mathrm{mV} / \mathrm{ms}, \psi_{0}=5 \mathrm{mV}$. 
Since the threshold time constant $\tau_{\psi}$ is typically much larger than the membrane time constant, the threshold dynamics can be approximated by:

$$
\psi(t)=A c \tau_{M} \frac{\left(1-\tau_{M} \tau_{\psi} \omega^{2}\right) \cos (\omega t)+\left(\tau_{M}+\tau_{\psi}\right) \omega \sin (\omega t)}{\left(1+\tau_{M}^{2} \omega^{2}\right)\left(1+\tau_{\psi}^{2} \omega^{2}\right)},
$$

which is a twice low-pass filtered cosine, where the filter frequencies are given by the inverse membrane time constant $\tau_{M}^{-1}$ and the inverse threshold time constant $\tau_{\psi}^{1}$.

Evaluating the integral Eq. (5.12) with $V(t) \rightarrow V(t)+f(t)$ and the time-dependent threshold Eq. (5.6), yields:

$$
\begin{aligned}
v_{T D}(t)=\left(2 \pi \sigma_{V}\right)^{-1} \exp \left(-\frac{(f(t)-\psi(t))^{2}}{2 \sigma_{V}^{2}}\right) \\
\\
\cdot\left\{\sigma_{\dot{V}} \exp \left(-\frac{\dot{f}(t)^{2}}{2 \sigma_{\dot{V}}^{2}}\right)+\sqrt{\pi / 2} \dot{f}(t)\left(1+\operatorname{erf}\left(\dot{f}(t) /\left(\sqrt{2} \sigma_{\dot{V}}\right)\right)\right)\right\}
\end{aligned}
$$

Fig. 5.5 shows the response for three different modulation frequencies and compares it with direct numerical simulations. For very slow input modulations $\left(\omega \ll \tau_{\psi}^{-1}\right)$, the threshold faithfully follows the MP modulations, i.e. $\psi(t)=f(t)$. As $\dot{f}(t)$ is negligible in this case, the rate becomes timeindependent:

$$
\lim _{\omega \rightarrow 0} v_{T D}(t)=\frac{\sigma_{\dot{V}}}{2 \pi \sigma_{V}} \exp \left(-\frac{\psi_{0}^{2}}{2 \sigma_{V}^{2}}\right)
$$

If the threshold variability is reduced by decreasing the coupling between the MP and the dynamic threshold, the suppression of the response at low frequencies is lost.

For very fast input modulations $\left(\omega \gg \tau_{c}^{-1}\right)$, the threshold $\psi(t)$ and the low-passed signal $f(t)$ are approximately constant, while the amplitude of the temporal derivative $\dot{f}(t)$ becomes independent of $\omega$, leading to a large response to larger frequencies. This is reflected in the raster plots in Fig. 5.5B. Although an individual neuron fires on average with a low rate of less than $5 \mathrm{~Hz}$, APs are time locked to the phase of the stimulation. To study the transmission properties of the population response for intermediate frequencies systematically, I will now investigate the linear response properties.

\subsubsection{Linear response theory}

To characterize the response in the linear response regime, I expand $v_{T D}(t)$ in powers of $A$ :

$$
v(t)=v_{0}+A v_{1}(\omega) \cos (\omega t+\varphi)+\mathscr{O}\left(A^{2}\right)
$$

The stationary rate $v_{0}$, the transmission function $v_{1}(\omega)$ and the relative phase shift are given by:

$$
v_{0}=\frac{\sigma_{V}}{2 \pi \sigma_{\dot{V}}} \exp \left(-\frac{\psi_{0}^{2}}{2 \sigma_{V}^{2}}\right)
$$




\section{Functional consequences of anomalous action potential initiation: An exactly solvable model}
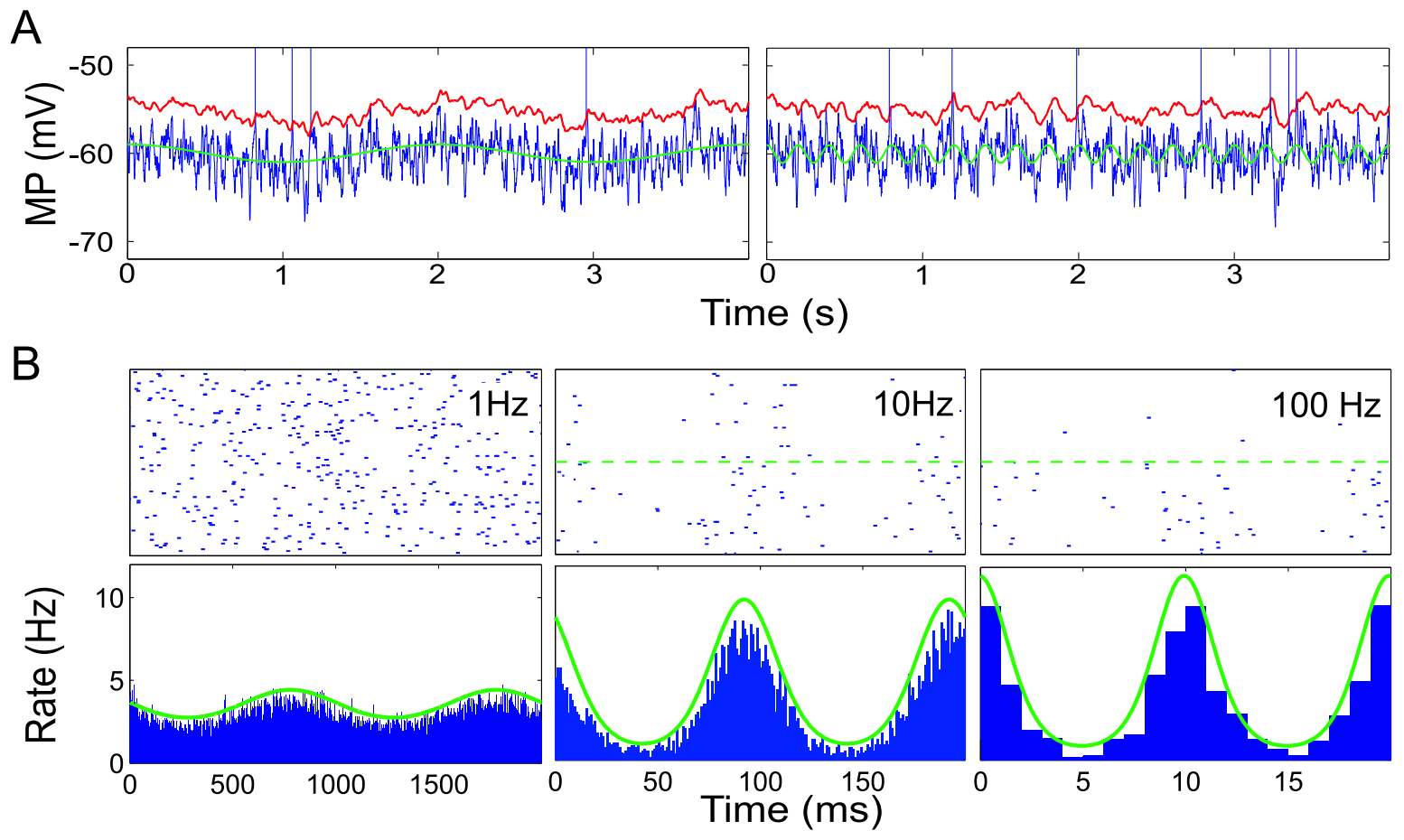

Figure 5.5: (A) MP (blue) and dynamic threshold (right) of the $V-\psi$ model in response to a cosinestimulus (green) with a modulation frequency of $1 \mathrm{~Hz}$ (left panel) and $10 \mathrm{~Hz}$ (right panel). (B) Firing rate response of an ensemble of neurons to a $1 \mathrm{~Hz}, 10 \mathrm{~Hz}$ and $100 \mathrm{~Hz}$ stimulus. Upper panels: Raster plots of 100 neurons. The green dashed line separates raster plots of 50 randomly selected neurons (above the line) from 50 neurons which generate at least one AP in the displayed time interval (below the line). Lower panels: Instantaneous firing rate (1 ms bins) averaged of 10000 independent neurons. Green curve: Firing rate in the limit $\tau_{\psi} \gg \omega^{-1}$.

Already for a modulation amplitude of $10 \mathrm{~Hz}$, the threshold dynamics does not follow the stimulation. The ensemble rate, however, phase locks to the stimulation. The amplitude of the modulation even grows with increasing frequencies. This shows that a variable threshold results in a high-pass filter characteristics. Parameters: $\sigma_{V}=2 \mathrm{mV}, \sigma_{\dot{V}}=1 \mathrm{mV} / \mathrm{ms}, \psi_{0}=5 \mathrm{mV}, \tau_{\psi}=30 \mathrm{~ms}$ 


$$
\begin{aligned}
& v_{1}(\omega)= \exp \left(-\frac{\psi_{0}^{2}}{2 \sigma_{V}^{2}}\right) \\
& \cdot \frac{\left(\pi \omega^{2}\left(1+\tau_{\psi}^{2} \omega^{2}\right) \sigma_{V}^{4}+2 \psi_{0} \sigma_{\dot{V}}\left(\sqrt{2 \pi} c \tau_{\psi} \omega^{2} \sigma_{V}^{2}+\psi_{0}\left((c-1)^{2}+\tau_{\psi}^{2} \omega^{2}\right) \sigma_{\dot{V}}\right)\right)^{1 / 2}}{\sqrt{8} \pi \sigma_{V}^{3}\left(\left(1+\tau_{M}^{2} \omega^{2}\right)\left(1+\tau_{\psi}^{2} \omega^{2}\right)\right)^{1 / 2}} \\
& \varphi(\omega)=\arctan \left(-\frac{\omega\left(\sqrt{2 \pi}\left(1+\tau_{\psi}^{2} \omega^{2}\right) \sigma_{V}^{2}+2 \psi_{0}\left(c \tau_{\psi}+\tau_{M}\left(c-1-\tau_{\psi}^{2} \omega^{2}\right)\right) \sigma_{\dot{V}}\right)}{\sqrt{2 \pi} \tau_{M} \omega^{2}\left(1+\tau_{\psi}^{2} \omega^{2}\right) \sigma_{V}^{2}+2 \psi_{0}\left(1-c+\tau_{\psi}\left(c \tau_{M}+\tau_{\psi}\right) \omega^{2}\right) \sigma_{\dot{V}}}\right)
\end{aligned}
$$

In the limit $\omega \rightarrow \infty$, the amplitude of the rate modulation $v_{1}(\omega)$ becomes constant and independent of $c$ :

$$
\lim _{\omega \rightarrow \infty} v_{1}(\omega)=\left(\sqrt{8 \pi} \tau_{M} \sigma_{V}\right)^{-1} \exp \left(-\frac{\psi_{0}^{2}}{2 \sigma_{V}^{2}}\right)
$$

The relative phase lag goes to zero:

$$
\lim _{\omega \rightarrow \infty} \varphi(\omega)=0
$$

This means that even in linear response, the model responds with a finite amplitude to modulations with very high frequencies, although the modulation amplitude of $f(t)$ and $\psi(t)$ goes to zero in the limit $\omega \rightarrow \infty$. This counterintuitive result has the same origin as the artificial response behavior of IF models, which I discussed in chapter 3 . It is a direct consequence of a sharp threshold for AP initiation.

I would like to point out that the transmission function $v_{1}(\omega)$ will naturally exhibit a cut-off frequency for large frequencies, if AP initiation is modeled more realistically. In this case, the same universal high-frequency limit, as discussed in Sec. 3.8 will apply and lead to a $\omega^{-1}$ decay for large frequency modulations.

In the limit $\omega \rightarrow 0$, the response function is given by:

$$
v_{1}(0)=\sqrt{2 / \pi}(c-1) \frac{\psi_{0} \tau_{M} \sigma_{\dot{V}}}{\sigma_{V}^{2}} v_{1}(\infty)
$$

Note that for a complete coupling of the threshold to the MP (i.e. $c=1$ ), the transmission function $v_{1}(0)$ is zero. In this case the threshold just follows the MP adiabatically, thus suppressing a modulation of the time-dependent firing rate.

The phase $\varphi(\omega)$ goes to zero, except for the case $c=1$, where it goes to $-\pi / 2$. The transition between the two limiting regimes $\omega \rightarrow 0$ and $\omega \rightarrow \infty$ is given by the full form of $v_{1}(\omega)$ from Eq. (5.31), as displayed in Fig. 5.6. The transmission functions for different values of the stationary firing rate and coupling constants exhibit a maximum, which is approximately at $\tau_{\psi}^{-1}$. The phase typically exhibits two maxima. The phase first advances the driving up to the frequency at which the transmission exhibits a maximum. It then lags behind the driving and goes to zero for large frequencies.

For $c=1$, the transmission function goes to zero for $\omega \rightarrow 0$ and the phase to $-\pi / 2$, as predicted by Eq. (5.35). For larger values of $c$, the overall transmission function shifts to larger amplitudes, the overall shape, however, does not change. Taken together, the suppression of low frequency modulation for large values of the coupling constant in combination with the facilitation of the response at high frequencies equips this neuron model with a high-pass filter characteristics. 


\section{Functional consequences of anomalous action potential initiation: An exactly solvable model}
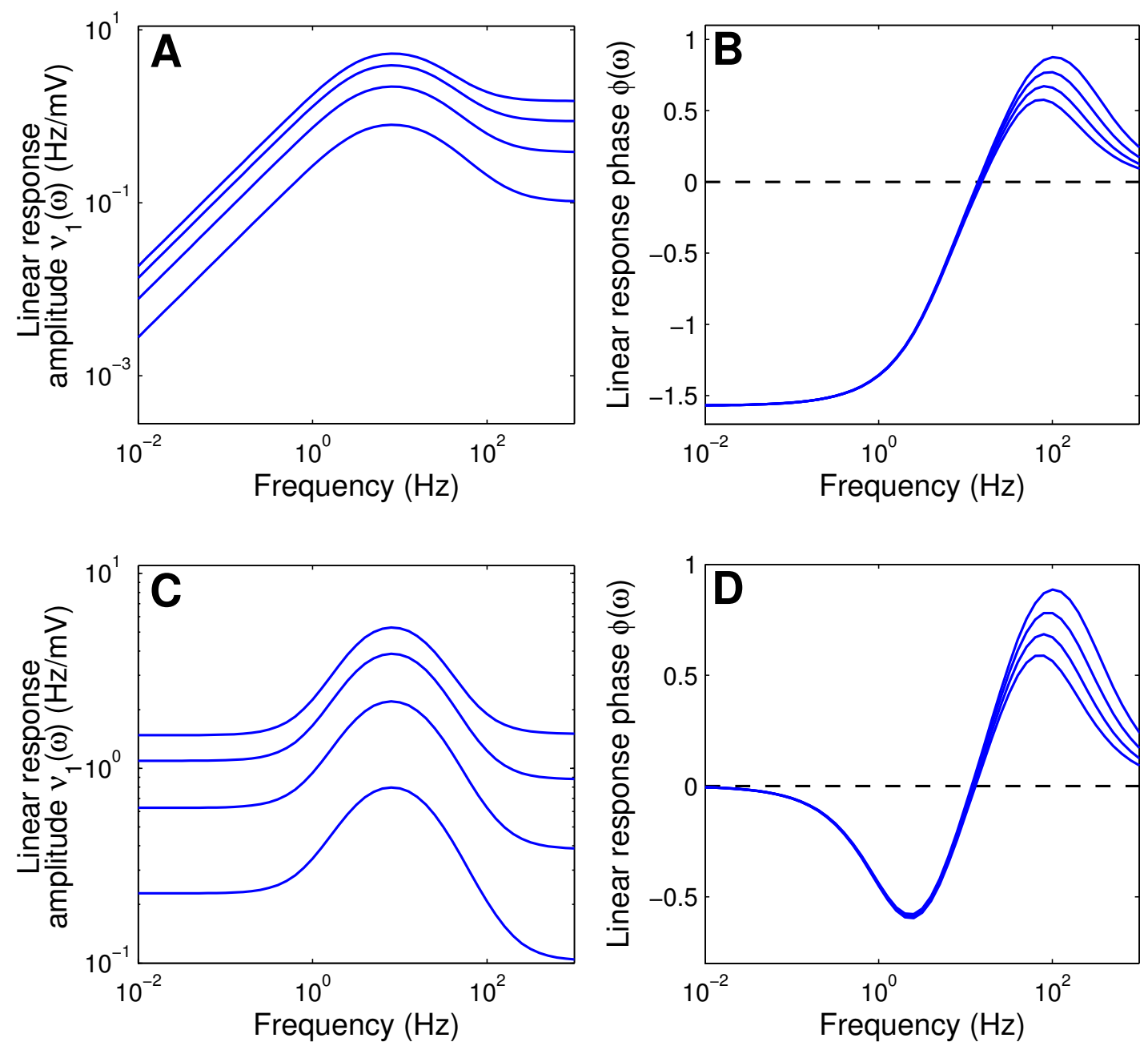

Figure 5.6: Linear response transmission function and phase shift of the $V-\psi$ model. (A) Transmission amplitude $v_{1}(\omega)$ and (B) phase for the case of a fully coupled threshold $(c=1)$ and different stationary firing rates $(1 \mathrm{~Hz}, 5 \mathrm{~Hz}, 15 \mathrm{~Hz}, 30 \mathrm{~Hz})$. (C,D) Transmission amplitude and phase for a partially coupled threshold $(c=0.75)$.

The transmissions functions exhibit a pronounced resonance and settle on a finite value for $\omega \rightarrow \infty$. For the case of a fully coupled threshold, the transmissions function goes to zero for $\omega \rightarrow 0$. Parameters: $\sigma_{V}=1.5,1.75,2.0,2.25 \mathrm{mV}, \sigma_{\dot{V}}=1 \mathrm{mV} / \mathrm{ms}, \tau_{M}=5 \mathrm{~ms}, \tau_{\psi}=30 \mathrm{~ms}$ and $\psi_{0}=5 \mathrm{mV}$. 


\section{$5.7 V-\dot{V}$ correlation at action potential onset}

\subsubsection{An alternative mechanism to explain the AP onset variability in vivo}

For cortical neurons from visual cortex, an alternative mechanism for the variability of onset potentials was proposed (Azouz \& Gray, 2000, 2003). The authors of these studies showed that the onset potentials of APs in vivo is correlated with the MP velocity at AP onset. In their recordings, the threshold for AP initiation was lower when the membrane depolarized rapidly and higher when the membrane depolarized slowly (see Fig. 5.7). From this result, the authors concluded that there is a combined threshold of the MP and its velocity. Shortly after the study had appeared, a correlation between AP onset potentials and the velocity was confirmed for hippocampal neurons (Henze \& Buzsáki, 2001).

\subsection{2 $V-\dot{V}$ correlation in the $V-\psi$ model}

Could the $V-\dot{V}$ correlation at AP onset be a consequence of a subthreshold adaptation mechanism instead of representing a mixed $V-\dot{V}$ threshold? To answer this question one has to look at the pdf $P(\psi, \dot{V})$ of the threshold and the MP velocity. In the $V-\psi$ model, this quantity is given by:

$$
P(\psi, \dot{V})=\exp \left(-\frac{1}{2}\left(\left(\begin{array}{c}
\psi-\psi_{0} \\
\dot{V}
\end{array}\right)\left(\begin{array}{cc}
\left\langle\psi^{2}-\psi_{0}^{2}\right\rangle & \langle\psi \dot{V}\rangle \\
\langle\psi \dot{V}\rangle & \left\langle\dot{V}^{2}\right\rangle
\end{array}\right)^{-1}\left(\begin{array}{c}
\psi-\psi_{0} \\
\dot{V}
\end{array}\right)\right)\right)
$$

where the explicit time index was omitted. Maximizing the exponent for every value of $\dot{V}$ gives a linear relation between $\psi$ and $\dot{V}$ :

$$
\psi=-c \frac{\Sigma^{2} \tau_{\psi}}{\Sigma^{2}+3 \Sigma \tau_{\psi} / \sqrt{2}+\tau_{\psi}^{2}} \dot{V}+\psi_{0},
$$

with $\Sigma=\sigma_{V} / \sigma_{\dot{V}}$. This implies that the observed correlation between the AP onset potential and the MP velocity may indeed be a direct consequence of a subthreshold adaptation of the AP onset potential in in vivo recordings of cortical neurons. A consequence would be that the correlation line given by Eq. (5.37) is not a threshold for AP initiation. In Fig. 5.8 the pdf $P(\psi, \dot{V})$ is depicted together with the linear relation Eq. (5.37) and subthreshold MP trajectories which cross the "threshold"-line without leading to an AP. In this example, the subthreshold crosses the correlation line approx. 20 times more often than predicted by the neurons' stationary rate, which is typical in the model for many parameter values tested.

\subsection{Gaussian neuron with a threshold in the $V-\dot{V}$ plane}

The example above showed that there can be a large mismatch between the prediction of APs from a moving threshold and from a threshold in the $V-\dot{V}$ plane. The formalism of the Gaussian neuron model directly allows to calculate the rate in the case of a threshold in $V-\dot{V}$ plane and compare the rates for both models systematically. 
A

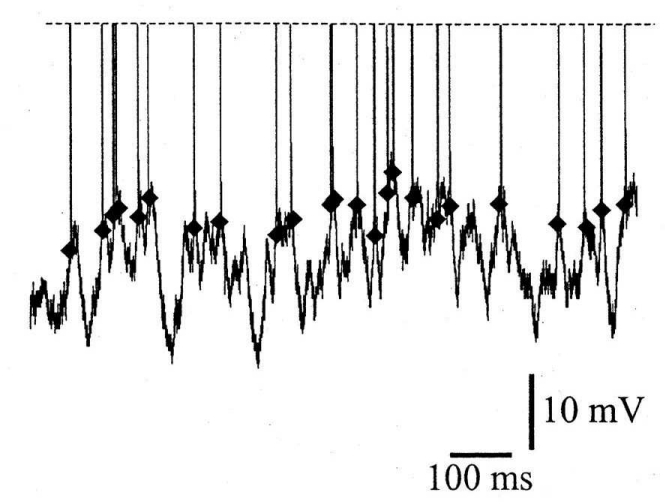

$\mathrm{C}$

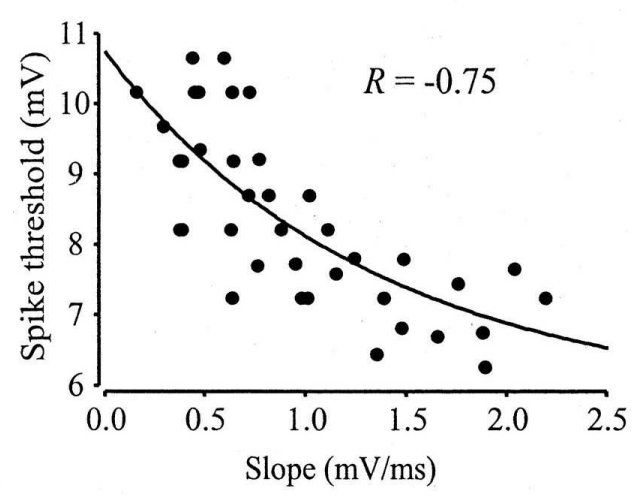

B

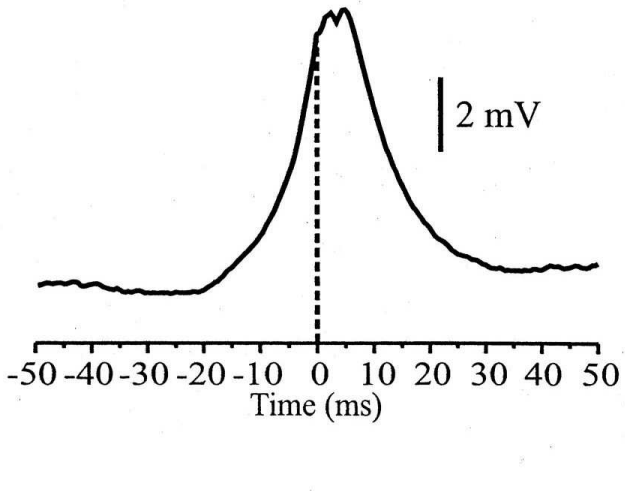

$\mathrm{D}$

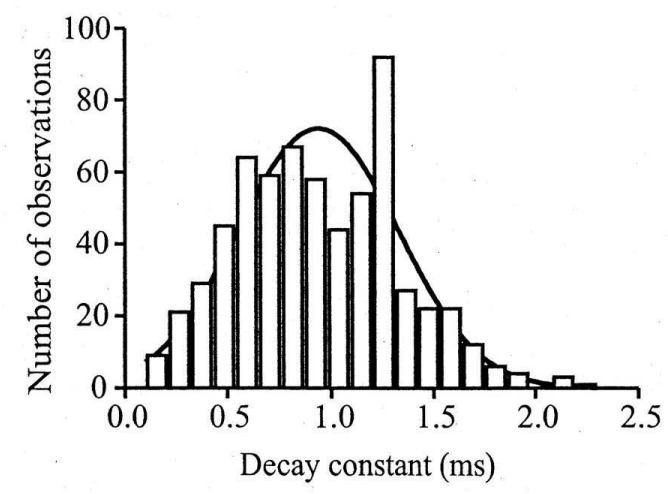

Figure 5.7: Spike threshold is inversely correlated with the rate of membrane depolarization. (A) Example of the raw data collected from an intrinsic bursting cell during the response to a drifting grating. (B) Spike-triggered average of the MP for all spikes occurring during the presentation of the visual stimulus. The dashed line corresponds to the time of AP generation. The mean rise time (from baseline to peak) for this cell was $12 \mathrm{~ms}$. (C) Scatter plot of dV/dt preceding a spike vs. spike threshold. The data in $\mathrm{C}$ were fit by the equation $V_{\mathrm{th}}=a+b \exp \left(-\dot{V}_{\mathrm{th}} / c\right)$. (D) Histogram of the decay constants $c$, obtained from all trials that were significantly fit by the equation in (C). (Adapted from Azouz \& Gray (2000)) 


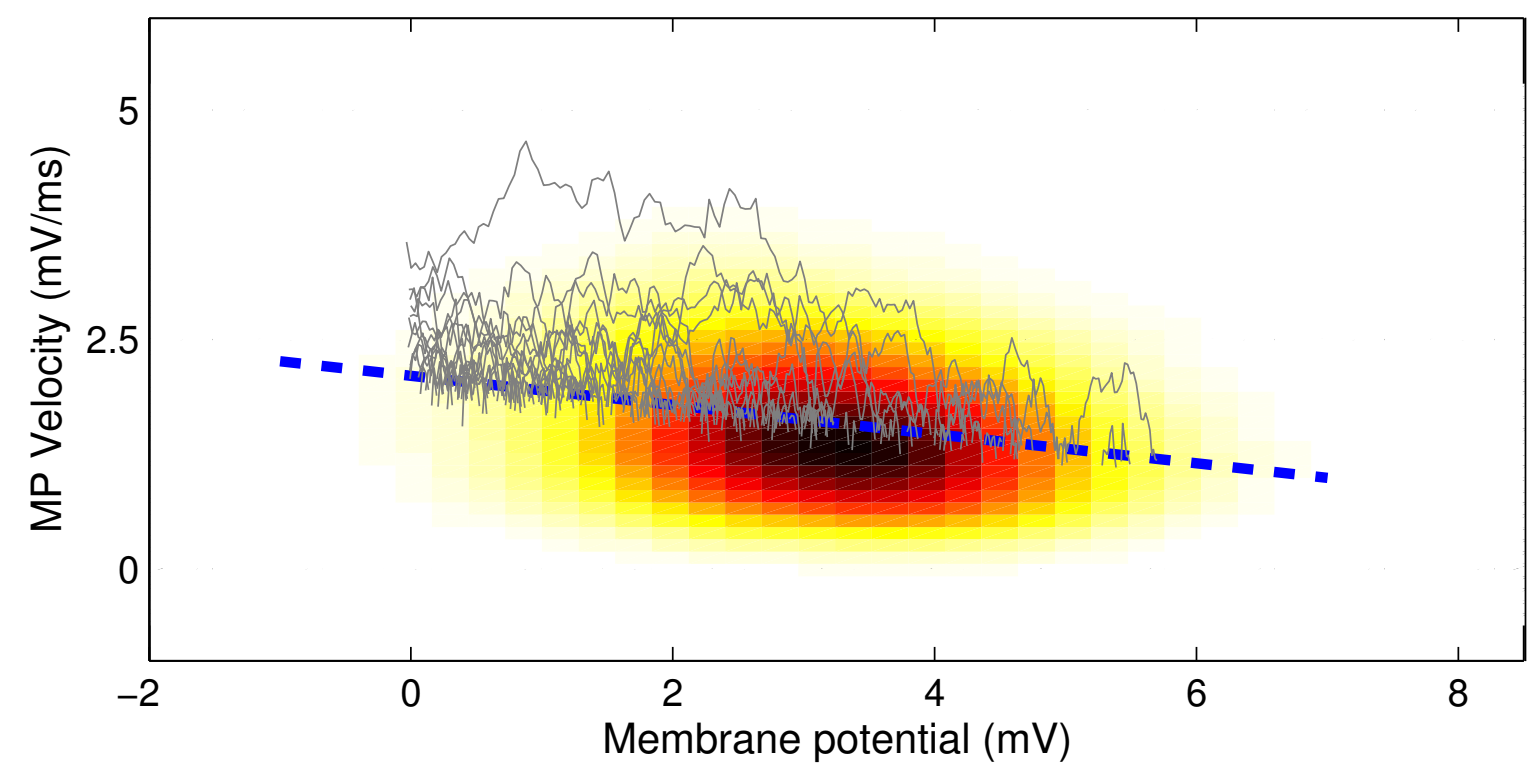

Figure 5.8: Is a threshold in the phase plane a good predictor for AP initiation? The conditional pdf $P(\psi \mid \dot{V})$ (color coded) and its major axis (dashed line), which is the putative threshold for AP initiation, is shown. Gray trajectories: Parts of simulated MP trace of 1s length which cross the correlation line. The stationary rate of the neuron was chosen as $12 \mathrm{~Hz}$. In this example, there are approx. 20 times as many crossings of the "threshold" than emitted APs. $\sigma_{V}=2 \mathrm{mV}, \sigma_{\dot{V}}=1 \mathrm{mV} / \mathrm{ms}$, $\psi_{0}=3.5 \mathrm{mV}, \tau_{\psi}=15 \mathrm{~ms}$ 


\section{Functional consequences of anomalous action potential initiation: An exactly solvable model}

As before, the stationary rate is given by an ensemble average of the number of threshold crossings:

$$
\begin{aligned}
v= & \int_{-\infty}^{\infty} d V d \dot{V} d \ddot{V} \delta(f(V, \dot{V})) \\
& \left|\frac{\partial f}{\partial V} \dot{V}+\frac{\partial f}{\partial \dot{V}} \ddot{V}\right| \Theta(\dot{V}) P(V, \dot{V}, \ddot{V}), \\
= & \frac{1}{2} \int_{-\infty}^{\infty} d V d \ddot{V}|a(a V+b)-\ddot{V}| P(V, a V+b, \ddot{V}),
\end{aligned}
$$

where $f(V(t), \dot{V}(t))=a V(t)+b-\dot{V}(t)$ parameterizes the linear threshold. The kernel of the integral now incorporates the second derivative of the MP $\ddot{V}(t)$ and thus its statistics has to be included into the density:

$$
P(V, \dot{V}, \ddot{V})=\left((2 \pi)^{3 / 2} \operatorname{det} \mathbf{C}_{\mathbf{v} \dot{\mathbf{v}}}\right)^{-1} \exp \left(-\frac{1}{2}\left(\begin{array}{c}
V-V_{0} \\
\dot{V} \\
\ddot{V}
\end{array}\right)^{T} \mathbf{C}_{\mathbf{v} \dot{\mathbf{v}}}{ }^{-1}\left(\begin{array}{c}
V-V_{0} \\
\dot{V} \\
\ddot{V}
\end{array}\right)\right)
$$

with the correlation matrix:

$$
\mathbf{C}_{\mathbf{V} \dot{\mathbf{V}}}=\left(\begin{array}{ccc}
\sigma_{V}^{2} & 0 & -\sigma_{\dot{V}}^{2} \\
0 & \sigma_{\dot{V}}^{2} & 0 \\
-\sigma_{\dot{V}}^{2} & 0 & \sigma_{\ddot{V}}^{2}
\end{array}\right)
$$

Fig. 5.9 shows the dependence of the firing rate on the variance of $\ddot{V}(t)$ for the Gaussian neuron model with a constant voltage threshold, an adapting threshold and a threshold in the $V-\dot{V}$ plane. The plot starts at a finite value of $\sigma_{\tilde{V}}^{2}$, as this variance is bounded from below, which is easily derived using Jensen's inequality:

$$
\sigma_{\ddot{V}}^{2} \geq \sigma_{\dot{V}}^{4} / \sigma_{V}^{2}
$$

Unexpectedly, the firing rate of the $V-\dot{V}$ threshold model increases linearly with $\sigma_{\tilde{V}}^{2}$, while it stays constant for the other two models. Moreover, the firing rate of this model is typically above the firing rates of the two other models. For realistic values of $\sigma_{\ddot{V}} \approx 3$, the $V-\dot{V}$ threshold model even predicts a firing rate which is larger by a factor of 20 .

This huge discrepancy in the predicted firing rate allows to assess for real neurons whether AP initiation is well described by a threshold in the $V-\dot{V}$ plane, or if the observed correlation in the $V-\dot{V}$ plane is merely a consequence of the subthreshold adaptation mechanism. Thus, in the following section, I directly test the assumption that a threshold in the $V-\dot{V}$ plane is a predictor for the generation of APs for in vivo intracellular recordings.

\subsection{Comparison with in vivo recordings}

In the previous section I showed that the assumption of a slowly varying threshold for AP initiation can lead to a $V-\dot{V}$ correlation at AP onset. I further showed that in the $V-\psi$ model, this correlation line does not "predict" the occurrence of APs in the sense of a threshold crossing, as conjectured on the basis of in vivo recordings (Azouz \& Gray, 2000, 2003). For realistic parameter values, it even predicts firing rates which are by orders of magnitude larger than for the threshold adaptation model. 


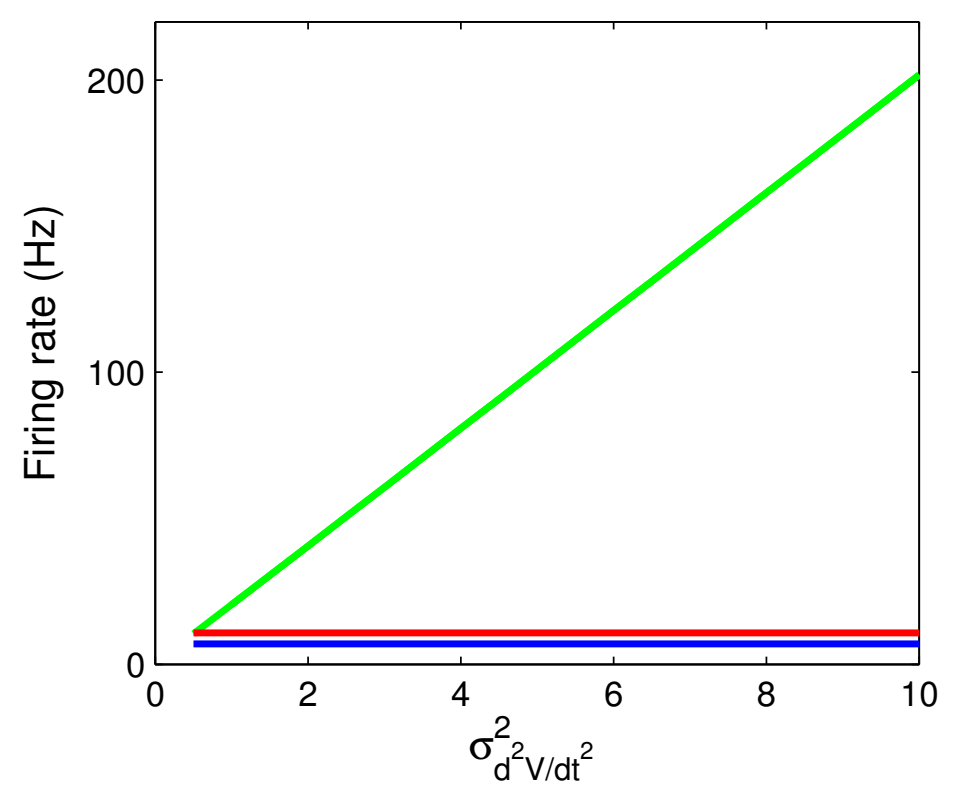

Figure 5.9: Stationary firing rate dependence of the $V-\psi$ model (blue), with fixed threshold (red) and with a threshold in the $V-\dot{V}$ plane (green) on $\sigma_{\tilde{V}}^{2}$. While the firing rate is practically independent of $\sigma_{\vec{V}}$ in the model with a fixed threshold, as well as in the $V-\psi$ model, it increases linearly in a model which assumes a threshold for AP initiation in the $V-\dot{V}$ plane. Parameters: $\sigma_{V}=2 \mathrm{mV}$, $\sigma_{\dot{V}}=1 \mathrm{mV} / \mathrm{ms}, \psi_{0}=4 \mathrm{mV}, a=-0.17, b=2.12$. The parameters $a$ and $b$ are the parameters from the $V-\dot{V}$ correlation line.

In this section, I directly test if a threshold in the $V-\dot{V}$ plane is a good predictor for AP initiation.

\subsubsection{Methods}

In vivo intracellular recordings from 27 cat visual cortex pyramidal cells were separated into stimulus conditions leading to a high and low firing rate and a non-stimulus condition (spontaneous activity). Only stationary recordings were analyzed (see Sec. 4.3 and Sec. 4.5.2). For each AP in a recording, the time when it reached the maximum was determined by quadratic interpolation. The set of times of all AP maxima in a recording will in the following be denoted by $\left\{t_{i}\right\}$.

To study the statistics of the MP leading to the generation of APs, all APs were aligned with respect to their maxima. For a range from $0.5 \mathrm{~ms}$ and $5 \mathrm{~ms}$ prior AP maximum, the linear correlation coefficient $r(\tau)$ between the set $\left\{V\left(t_{i}-\tau\right)\right\}$ and $\left\{\dot{V}\left(t_{i}-\tau\right)\right\}$ as a function of $\tau$ was calculated. It turned out that for each stimulus condition, $r(\tau)$ exhibited a maximum at times $\tau_{\text {opt }}$ at which $V(t)$ and $\dot{V}(t)$ were maximally statistically dependent.

Then, the linear regression line through the set of $\left\{V\left(\tau_{\text {opt }}\right)\right\}$ and $\left\{\dot{V}\left(\tau_{\text {opt }}\right)\right\}$ was determined at the offset $\tau_{\text {opt }}$ :

$$
\dot{V}\left(\tau_{\mathrm{opt}}\right)=a V\left(\tau_{\mathrm{opt}}\right)+b,
$$




\section{Functional consequences of anomalous action potential initiation: An exactly solvable model}

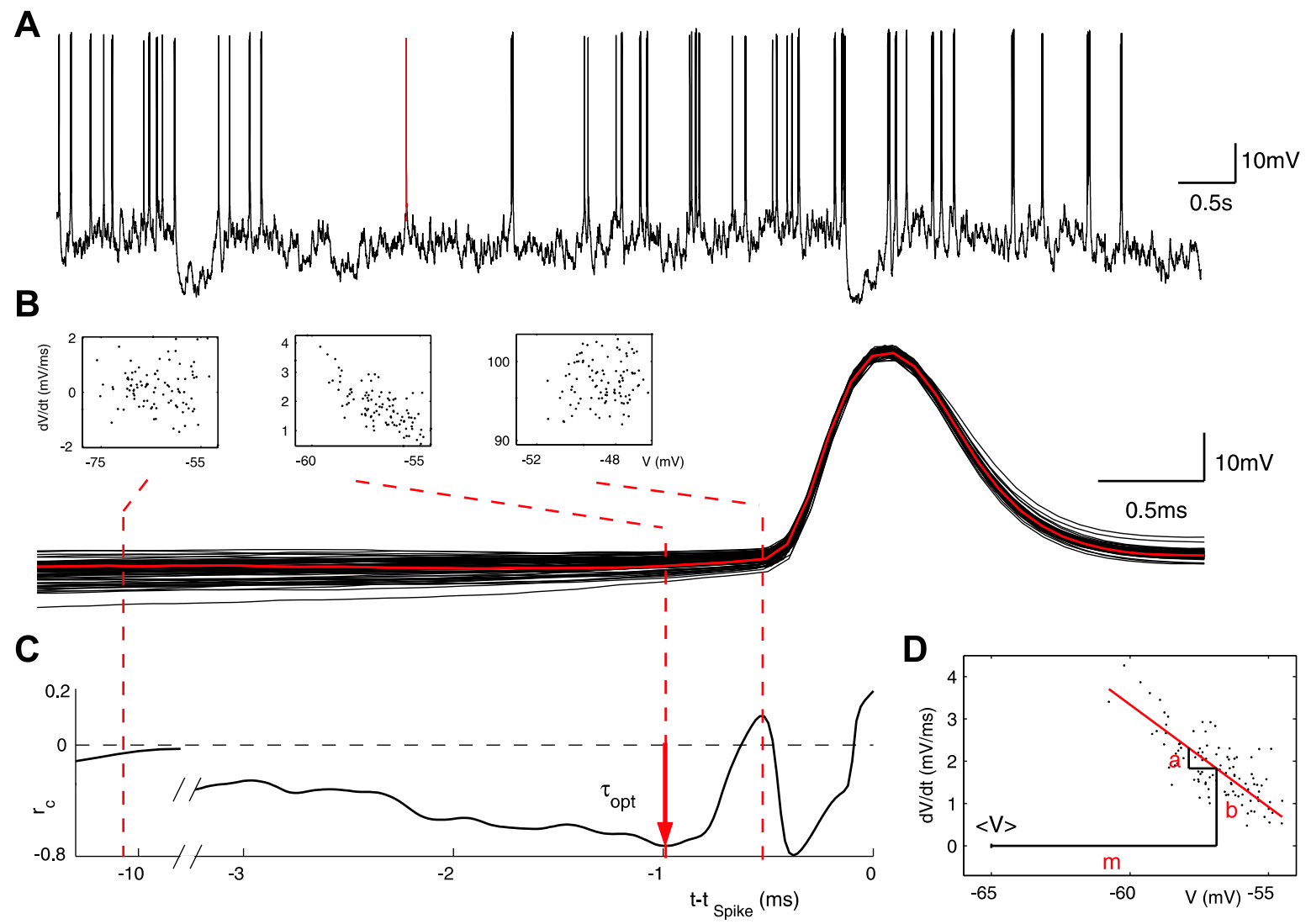

Figure 5.10: Data analysis method to determine $V-\dot{V}$ correlation. (A) Intracellular in vivo recording from a visual cortical neuron showing spontaneous activity. (B) All APs from (A) are superimposed such that their maxima become aligned. The insets are scatter plots of the MP $V(t)$ vs. its velocity $\dot{V}(t)$ for different temporal offsets (denoted by the dashed lines). While $V$ and $\dot{V}$ are practically uncorrelated at AP onset and far away from AP onset, they are maximally correlated at a time inbetween, defining an optimal temporal offset $\tau_{\text {opt }}$. (C) Correlation coefficient $r(\tau)$ of the MP $V(t)$ and its velocity $\dot{V}(t)$. At $\tau_{\text {opt }}$, the correlation coefficient exhibits a minimum. (D) At $\tau=\tau_{\text {opt }}$, a linear regression line with offset $b$ and slope $a$ is determined. 
where,

$$
\begin{aligned}
& a=(\langle V \dot{V}\rangle-\langle V\rangle\langle\dot{V}\rangle) /\left(\left\langle V^{2}\right\rangle-\langle V\rangle^{2}\right) \\
& b=\left(\langle\dot{V}\rangle\left\langle V^{2}\right\rangle-\langle V\rangle\left\langle\dot{V}^{2}\right\rangle\right) /\left(\left\langle V^{2}\right\rangle-\langle V\rangle^{2}\right) .
\end{aligned}
$$

From the correlation matrix, the $2 \sigma$-correlation ellipse was determined, in which $95 \%$ of all data points are expected to be, assuming a Gaussian distribution.

To assess, if in cortical neurons there is a $V-\dot{V}$ threshold for AP initiation, the number of trajectories which cross the correlation but do not lead to the emission of an AP within the next 10 ms (non-AP trajectories) was determined. To further characterize these trajectories, I also assessed the maximum distance which non-AP trajectories depart from the correlation line and the duration they spend above the correlation line. To measure the distance, the data in the $V-\dot{V}$ plane was first normalized by dividing their coordinates by the standard deviations of $\left\{V\left(t_{i}-\tau\right)\right\}$ and $\left\{\dot{V}_{i}\left(t_{i}-\tau\right)\right\}$, respectively. The distance was subsequently measured along the major axis of the $2 \sigma$-correlation ellipse which is perpendicular to the correlation line.

\subsubsection{Results: AP initiation in vivo is not a threshold process in the $V-\dot{V}$ plane}

For all traces, the temporal offset $\tau_{\mathrm{opt}}$, was detected reliably, it ranged from $0.5 \mathrm{~ms}$ to $3.9 \mathrm{~ms}$ with a mean value of $1.6 \mathrm{~ms}$. The correlation coefficient $r\left(\tau_{\mathrm{opt}}\right)$ ranged from -0.31 to -0.97 with a mean value of -0.65 .

How faithfully does the correlation ellipse predict the occurrence of APs? In Fig. 5.11A, a typical in vivo trace of spontaneous activity is shown. The assumption that the $V-\dot{V}$-correlation line is a threshold for AP initiation leads to many spurious APs (Fig. 5.11B). In the phase-plane representation, subthreshold trajectories which do not lead to an AP cross the correlation line and can even depart from it much further than the correlation ellipse (Fig. 5.11C). Fig. 5.11D shows all superimposed APs and the grey trajectories from Fig. 5.11C in the $V(t)-t$ space. The gray trajectories span the same voltage range as the AP trajectories and only divert once the AP has started.

In Fig. 5.12, the results are summarized for all in vivo traces and stimulus conditions analyzed. For most stimulus conditions, the correlation line in the $V-\dot{V}$-plane predicts firing rates which are by a factor of 2 larger than the measured firing rate, irrespective of the stimulus condition. For some stimulus conditions, the predicted rates are even larger by more than one order of magnitude. Moreover, the discrepancy between actual and predicted firing rate was larger for stimulus conditions exhibiting a low firing rates. For high firing rates above $20 \mathrm{~Hz}$, the predicted firing gets closer to the measured firing rate.

A reason for the large difference in predicted and actually measured firing rate could be due to non-AP trajectories which spend only a very short amount of time over the correlation line, which would be attributed to measurement noise. To test for this hypothesis, in Fig. 5.13, the durations, AP trajectories spend in the $2 \sigma$-correlation ellipse are compared with the durations non-AP trajectories spend over the correlation line. $40 \%$ of all non-AP trajectories spend more than $0.2 \mathrm{~ms}$ over the correlation line with no substantial difference between the different stimulus conditions. The duration which AP trajectories spent in the correlation ellipse is in $29 \%$ of all stimulus conditions larger than $0.2 \mathrm{~ms}$ with no qualitative difference between different stimulus conditions. This clearly demonstrates that 


\section{Functional consequences of anomalous action potential initiation: An exactly solvable model}
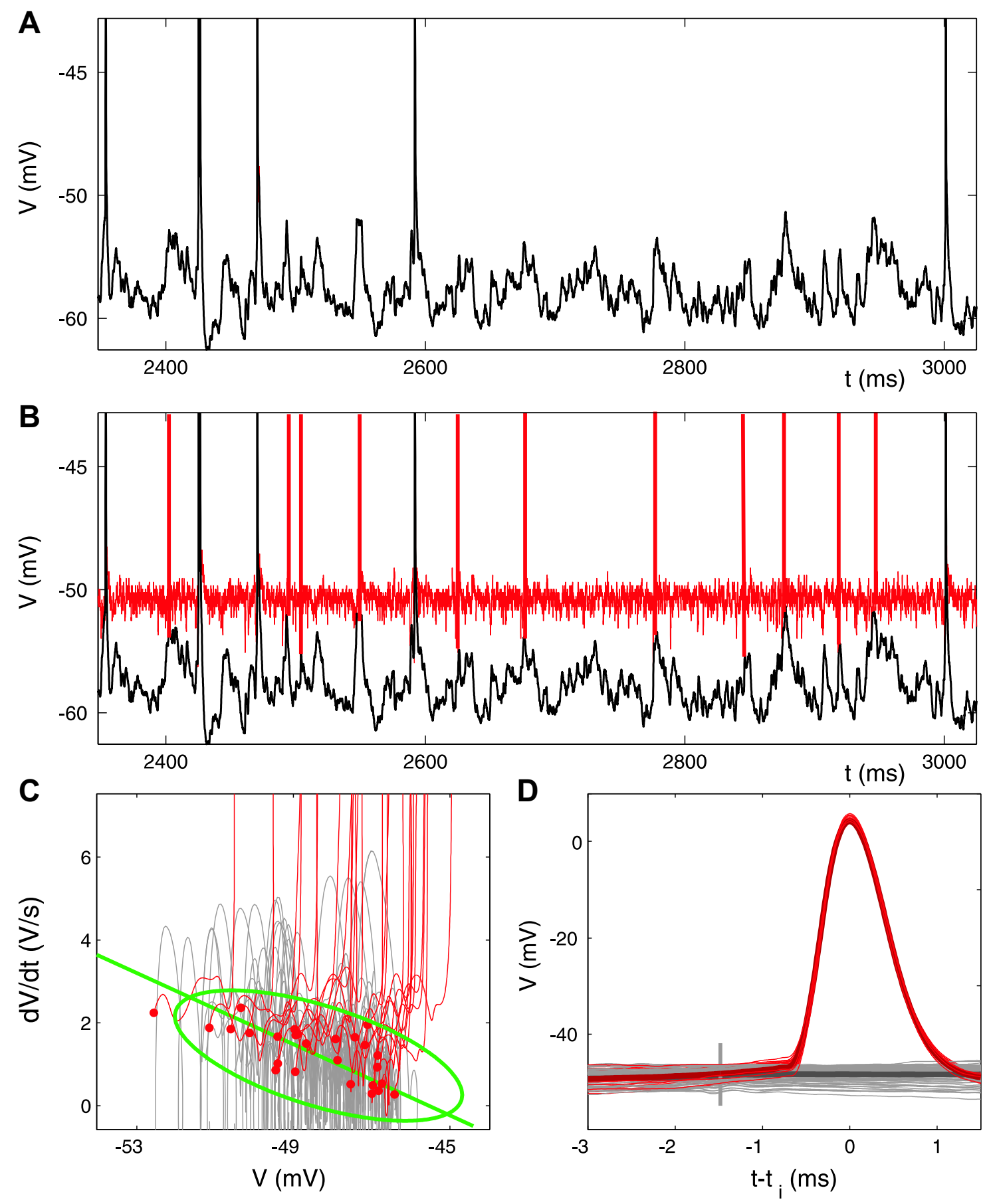

Figure 5.11: $V-\dot{V}$-correlation line is not a threshold for AP initiation in vivo. (A) Intracellular in vivo recording from a visual cortical neuron showing spontaneous activity. (B) Same trace as in (A) with moving $V-\dot{V}$ threshold (red). The $V-\dot{V}$-threshold predicts many spurious APs (red). (C) Phase plane representation of an in vivo trace. Magnification of the correlation ellipse and line (green), trajectories which cross the correlation line (gray), but do not lead to an AP, and AP trajectories with starting points (red). There are many non-AP trajectories, which can even depart far above the ellipse, demonstrating that the $V-\dot{V}$-correlation line is not a threshold for AP initiation. (D) Trajectories which do not lead to an AP, but cross the $V-\dot{V}$-correlation line (gray), superimposed at $\tau_{\text {opt }}$ (gray vertical line) and AP trajectories (red), superimposed with respect to their maxima. 


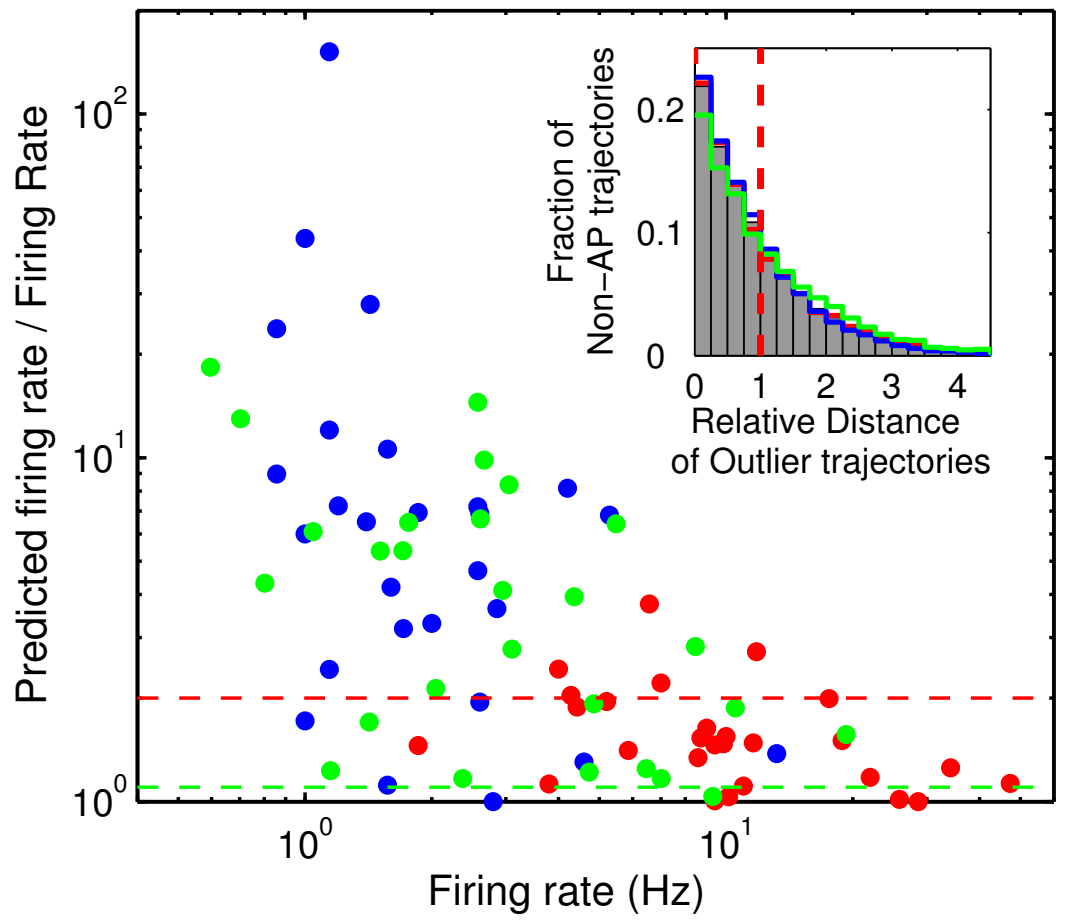

Figure 5.12: Ratio of predicted firing rate and actual firing rate vs. firing rate for all stimulus conditions and cells (green: low rate, red: high rate, green: spontaneous activity).

Green dashed line: $10 \%$ deviation of predicted rate from actual firing rate. Almost all data points $(93 \%)$ deviate more than $10 \%$ from the real firing rate (above green dashed line), most data points $(53 \%)$ deviate even more than $100 \%$ (red dashed line). Inset: Histogram showing the mean value of outlier distances for all recordings. On top, separate histograms for different stimulus conditions (same color code as in main panel). This value is larger than 1 for a substantial fraction of conditions (36\%), extending up to 4.5 . 


\section{Functional consequences of anomalous action potential initiation: An exactly solvable model}
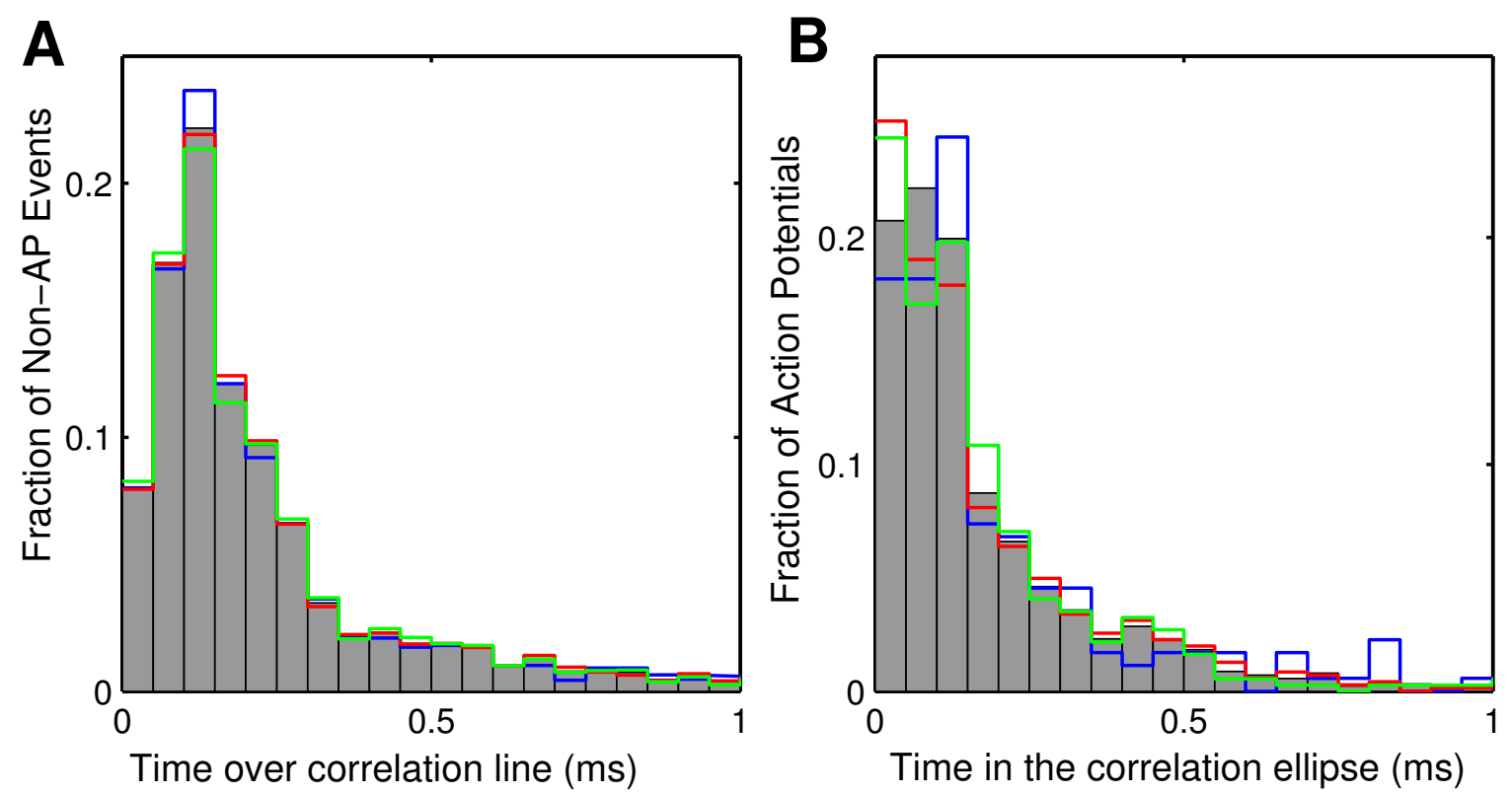

Figure 5.13: How long do AP and non-AP trajectories spend above the correlation line? (A) Histogram showing the time which non-AP trajectories stay over the correlation line. (B) Histogram showing the time which AP trajectories stay above the correlation line before leaving the $2 \sigma$ correlation ellipse. (Color code as in Fig. 5.12). Based on these histograms, both types of trajectories are indistinguishable.

the duration which trajectories spend over the correlation line does not distinguish AP from non-AP trajectories.

\subsection{Summary and discussion}

The dynamical consequences of a subthreshold adaptation mechanism were assessed in a novel phenomenological neuron model. This model is based on an experimentally observed correlation between AP onset potentials and the mean MP preceding it. Because of its simplicity, the model allowed to calculate many of its dynamic population coding properties analytically. I first showed that the modeled threshold adaptation mechanism leads to a reduction of the stationary firing rate. I then calculated the spike-triggered average MP (STA) which, surprisingly, exhibits an undershoot-like behavior. This undershoot was shown to vanish in the limit of an uncoupled threshold.

I then assessed the dynamic population response properties. It was shown that the combination of an adaptive threshold and a fast AP onset dynamics turns the population response into a high-pass filter, suppressing slowly varying input stimuli while rapid inputs are transmitted unattenuated. In the remainder of the chapter, I tested a previously proposed mechanism to explain the AP onset variability of cortical neurons in vivo. In this mechanism, it is assumed that an observed correlation between AP onset potentials and the velocity at AP onset serves as an effective threshold for AP initiation. It turns out that this correlation is a direct consequence of a subthreshold adaptation mechanism, but is not a threshold for AP initiation, which I tested in in vivo recordings. I showed that the firing rates which 
would be predicted by a $V-\dot{V}$ threshold are typically larger by factors between two and 200 than the real firing rates.

In previous theoretical and modeling studies, the effects of threshold adaptation were exclusively studied in models which assume spike-triggered adaptation currents (Tuckwell, 1978; Vasudevan \& Vittal, 1982; Wilbur \& Rinzel, 1983; Chacron et al., 2003; Benda \& Herz, 2003; Jolivet et al., 2004; Lindner \& Longtin, 2005). These models predict a strong correlation between the ISI preceding an AP and AP onset potentials. In particular, they predict a vanishing adaptation effect for very low firing rates, as the typical ISI becomes very large. Both predictions are not compatible with the analyzed experimental in vivo recordings, which exhibit for many recordings only a small correlation between AP onset potentials and the preceding ISI, and a large correlation with the preceding mean MP. Functionally, both adaptation mechanisms lead to a suppression of slowly varying signals. It might thus be that both mechanisms are present in cortical neurons and take effect in different dynamical regimes.

In the $V-\psi$ model, the subthreshold adaptation leads to an undershoot behavior of the STA. This undershoot was also observed in in vitro recordings of pyramidal neurons, in which fluctuating input currents were injected (Mainen \& Sejnowski, 1995). The authors of this study attributed it to a subthreshold oscillatory dynamics of the MP. Its was shown, however, that pyramidal neurons in the cortex typically exhibit Type-I excitability (Tateno et al., 2004), for which no intrinsic oscillatory behavior is expected. Thus, it might well be that the observed oscillatory dynamics is a fingerprint of the subthreshold adaptation mechanism. A similar STA time-course was observed in an independent experimental study in pyramidal neurons (Nowak et al., 1997).

The simplicity of the $V-\psi$ model enabled a complete analytical characterization of the dynamic response properties. To my knowledge, this is the only model in which it is possible to calculate the linear and non-linear population response properties in a closed analytical form in the presence of a realistically fluctuating MP. This analytical treatment was possible because the model does not incorporate a reset mechanism after the emission of a spike, which, however, does not have an effect in the regime analyzed. The dynamical flaw the model suffers from, as with all IF models, is a nondecaying transmission function in the limit $\omega \rightarrow \infty$ (cf. chapter 3). A way to circumvent this problem would be to incorporate the subthreshold adaptation into a model with a dynamic AP onset, such as the generalized $\theta$-neuron.

\subsection{Perspectives}

In this chapter, the experimentally observed subthreshold adaptation mechanism was assessed in a phenomenological neuron model. A central issue is of course to understand the biophysical origin of this mechanism. As discussed in the previous chapter, several candidate mechanisms could possibly explain the large variability of the AP onset potentials. Among them are phosphorylation or dynamical coupling of voltage-gated channels, various second messenger mechanisms or, so far, unidentified voltage-gated channels (e.g. a special potassium channel subtype) which could lower or raise the onset potentials. Future in vitro experiments, in which the chemical milieu of a neuron can be well controlled and in which individual channels can be selectively blocked pharmacologically, will have to elucidate the biophysical basis of this mechanism.

A second important issue concerns the question: Which neuron model faithfully translates synaptic input currents into sequences of emitted APs, best? A first step to answer this question was undertaken 


\section{Functional consequences of anomalous action potential initiation: An exactly solvable model}

in this chapter, where a phenomenological approach was used to model the dynamics of cortical neurons and to uncover the dynamical consequences for information processing in neuronal populations.

The straightforward extension to this is to develop a model systematically and to identify "features" which predict the occurrence and the exact timing of APs in cortical neurons. Indeed, first studies show that this approach can predict the occurrence and timing of APs with a high accuracy (Fröhlich et al., 2005). This approach facilitates Support Vector Machines, a data classification concept from machine learning (Schölkopf \& Smola, 2002). Using this method, it was possible to predict APs in a reduced data set with prediction accuracies of more than $90 \%$. This accuracy is much higher than all previous approaches, which tried to model the detailed biophysical dynamics of cortical neurons (Jolivet et al., 2004).

The further identification and analysis of these types of models for different types of cortical neurons (e.g. interneurons) will be an important prerequisite for the dynamically faithful modeling of neural networks and the identification of the emergent properties of neural computation. 


\section{Summary and outlook}

In this thesis, I investigated the action potential (AP) initiation dynamics in cortical neurons and its consequences for dynamical population coding in the cortex.

In chapter 3, I assessed the population coding properties of a reduced neuron model, a generalization of the $\theta$-neuron, a canonical model of Type-I excitable neuronal membranes, in an in vivo-like regime and compared the model predictions with recent experimental studies. As a key result, I derived, using a novel method, a close connection between the speed, with which a population of such neurons can transmit time-varying stimuli and the single-neurons' AP onset dynamics. Surprisingly, the speed with which a population can respond to time-varying stimuli can be independent of the neurons membrane time constant or stationary firing rate. If the AP onset speed is very rapid, fast signals up to $1 \mathrm{kHz}$ can be transmitted unattenuated, although each neuron in the population fires at a rate which can be lower by two orders of magnitude.

In chapter 4, I assessed the AP onset dynamics in in vivo and in vitro recordings of cortical pyramidal neurons. I showed that onset dynamics is characterized by two key features: A very rapid onset and a large variability of onset potentials. It turned out that both features are mutually exclusive in HodgkinHuxley type neuron models and, unexpectedly, that the AP initiation in cortical neurons is outside the range of behaviors of this model class. To explain the biophysical origin of the anomalous dynamics of cortical APs, I discussed three hypothetical mechanisms.

In chapter 5, I studied the consequences of the AP onset variability observed in in vivo recordings of cortical neurons in a novel phenomenological neuron model, the $V-\psi$ model. I showed that in many recordings a large fraction of the variability of the onset potentials is explained by a subthreshold adaptation mechanism. Studying the dynamic response properties of the $V-\psi$ model, for which I derived explicit analytical expressions, revealed that the key features of cortical AP initiation turn the dynamical population response into a high-pass filter. I further demonstrated that the subthreshold adaptation mechanism can explain a recently observed correlation between the MP and its velocity at AP onset in in vivo intracellular recordings of cortical neurons.

To study the dynamical response properties in chapter 3 , a generalization of the $\theta$-neuron was used, which is a canonical phase oscillator model of excitable neuronal membranes exhibiting Type-I excitability. To model an in vivo-like situation, a fluctuating synaptic input current was added to the model equations. Using the Fokker-Planck equation which describes the dynamics of the population response, I calculated both the stationary and dynamical response properties in linear response theory. As revealed by the coefficient of variation, the stationary response is characterized by irregular spiking activity, which is typical for the spiking activity of cortical neurons in vivo. I then derived the high frequency limit of the dynamical transmission function. It turned out that the decay of the transmission function in this limit is universal and independent of model details. Surprisingly, it qualitatively depends on the oscillator phase $\theta_{s}$ at which a spike is emitted: If at $\theta_{s}$ the dynamics is insensitive to external inputs, transmission functions decay as (i) $\omega^{-3}$ for the case of a modulation of a temporally uncorrelated synaptic input current and (ii) as $\omega^{-2}$ for a modulation of the mean input current in the 


\section{Summary and outlook}

presence of a temporally correlated and uncorrelated synaptic input current and in the case of a modulation of the amplitude of a correlated synaptic input current. If the insensitivity condition is lifted, all transmission functions decay as $\omega^{-1}$, which is the behavior reported for conductance-based neuron models.

Using a novel sparse matrix representation of the systems Fokker-Planck operator, I then computed the full transmission function for arbitrary frequencies and both types of input stimulation in three distinct dynamical regimes: subthreshold, critical and suprathreshold. Frequencies up to the stationary rate of the neuron are in general transmitted unattenuated. If the AP onset dynamics is slow, all transmission functions decay rapidly to zero for larger frequencies. If, however, the AP onset speed is increased, the onset of the decay can be shifted to very large frequencies up to $1 \mathrm{kHz}$, where the transmission amplitude for modulations of the noise amplitude is typically much larger than for a modulation in the mean input current.

Comparing these results with the dynamical population response properties of real neurons, I showed that the generalized $\theta$-neuron exhibits a similar response behavior: For both model and real neurons the critical frequency up to which stimuli can be transmitted unattenuated can be much larger than the neuronal membrane time constant or the stationary firing rate. Moreover, the model makes an important prediction: To dynamically encode rapidly varying stimuli in a population of neurons, the AP onset dynamics has to be very rapid.

To elucidate the population coding properties in the cortex, I thus investigated in chapter 4 the AP initiation dynamics in in vivo and in vitro recordings of cortical neurons. It turned out that their onset dynamics is characterized by two key features: A very rapid onset and a large variability of onset potentials. I then quantitatively compared the observed onset dynamics with simulations of conductance-based neuron models of cortical neurons. I first showed that in this class of models the initiation dynamics is limited by the activation of voltage-gated sodium channels. I then demonstrated that the two key features of cortical AP initiation are mutually exclusive in this class of models. Modeled APs either exhibit a rapid onset dynamics or they are initiated in a large voltage range, but never both together. This suggests that a basic assumption of the theory is violated qualitatively.

I then presented three hypothetical mechanisms, that may explain the anomalous dynamics of AP initiation in cortical neurons. The first hypothesis assumes that channel stochasticity, due to a finite number of channels in a neuronal membrane, can effectively lead to a fast and variable onset behavior. Although it turns out that channel stochasticity leads to an increased noisiness of the AP onset dynamics, it can not account for the experimentally observed dynamical features. In the second hypothesis, a cooperative coupling of sodium channels at the node of Ranvier was assumed, where sodium channels are densely packed and APs are assumed to be initiated. A phenomenological model revealed that this coupling can qualitatively account for the fast onset dynamics and large variability at AP onset. The third hypothesis assumes that the activation curve of sodium channels exhibits a non-canonical step at low voltages. I showed that even a simple, two-step kinetic scheme of the channel activation would suffice to implement this hypothesis and explain the fast initiation dynamics of cortical APs.

The analysis of the initiation dynamics of cortical neurons in vivo additionally revealed a subthreshold adaptation mechanism, which is characterized by a correlation between the mean membrane potential before an AP is emitted and the onset potential of this AP. In chapter 5, I assessed the consequences of this adaptation mechanism for the dynamical population coding in a new phenomenological neuron model, the $V-\psi$ model. In contrast to conventional neuron models, in which the dynamics is determined in terms of stochastic differential equations, this model is constructed by directly defining a stochastic process. This alternative approach allows to give explicit analytical expressions for many 
quantities which describe the stationary and dynamical responses of a population of such neurons. It turns out that the adaptation mechanism generally leads to an overall reduction of the stationary firing rate. I then calculated the spike-triggered membrane potential average (STA). Surprisingly, the STA is characterized by an undershoot behavior, although the neuronal dynamics only incorporates relaxation processes. I further showed that in the model, this behavior is exclusively due to the subthreshold adaptation mechanism and that it is also typically found in measurements of the STA in in vitro recordings of cortical neurons.

Then, the dynamical firing rate response of the $V-\psi$ model was assessed. I derived the response function for stimulations of arbitrary amplitude as well as in the linear response regime. In accordance with the result on the stationary response properties, I showed that the subthreshold adaptation leads to a reduction of the transmission function at low frequencies. Together with an increase of the transmission function at high frequencies, due to the rapid AP onset dynamics, it turned out that the overall population firing rate response has a high-pass filter characteristics.

These results are closely connected to the question whether a cortical neuron acts as an integrator or as a coincidence detector (Koch et al., 1995; König et al., 1996). The conventional view is that, for single neurons, the discrimination between these two functional modes is determined by the membrane time constant of the neuron. On the other hand, the results derived in this thesis suggest that the limiting factor for the speed of population coding is the action potential initiation dynamics. This dynamics turned out to be specifically tailored in cortical neurons for rapid processing of fast stimuli and for adaptation in response to slow stimuli.

In the last section, I assessed a previously proposed mechanism for AP initiation in cortical neurons, a threshold in phase space, i.e. in the combined space of the membrane potential and its first temporal derivative. This threshold is based on the observation that in in vivo recordings, one can find a strong correlation between the membrane potential and its velocity at AP onsets. I showed that in the $V-\psi$ model, this correlation is a direct consequence of the subthreshold adaptation mechanism and does not serve as a threshold for AP initiation. The membrane potential can cross the correlation line an order of magnitude more often than APs are generated. Because of the robustness of the prediction, I further tested if a correlation line in phase space is a threshold for AP initiation in in vivo recordings of cortical neurons. The analysis of these recordings confirms the model result: In many recordings, in particular in those which exhibited low firing rates, the predicted firing rates are an order of magnitude larger than the actual rates observed, indicating that AP initiation in cortical neurons involves processes with long time constants which are not captured by criteria that incorporate only local properties of the membrane potential.

The issues worked out in my thesis open several perspectives for future research. By studying the population coding in reduced neuron models, I predicted that there is a close relation between the AP onset dynamics and the population coding properties of cortical neurons. I further showed that APs in cortical neurons appear to be specifically tailored to initiate rapidly. Obviously, the next step is to systematically assess the connection between the AP onset dynamics and the population coding properties in in vitro recordings. A first step in this direction was started in collaboration with Dr. Clemens Boucsein from the Bernstein Center for Computational Neuroscience in Freiburg.

Recent theoretical studies proposed a different mechanism to overcome the time-scale imposed by the single neurons' membrane-time constant, and to allow a population of connected neurons to respond faithfully to rapid stimuli (van Vreeswijk \& Sompolinsky, 1996). This mechanism, is based on a balance of strong excitatory and inhibitory feedback connections between neurons in a population. 
The existence of this balanced state has not yet been shown and the results presented in this thesis question its necessity for rapid information processing in the cortex.

Nevertheless, feedback connections are obviously a key property of cortical architecture on all spatial scales. The matrix method developed in chapter 3, proved to be a very useful tool to study the dynamical response properties of populations of non-interacting neurons. This method can also be extended to describe the dynamics of networks of interconnected neurons. Thus, the further investigation of the influence of feedback connections on the dynamical population coding properties of the neuron models discussed in this thesis appears to be a promising extension of the results presented.

A very crucial issue that needs to be addressed in future studies concerns the biophysical basis of AP initiation. It came quite as a surprise when we found that the classical Hodgkin-Huxley theory does not correctly describe the AP initiation in cortical neurons, as this process is central for the dynamical information processing in the cortex. I presented two hypothetical mechanisms which could explain the observed behavior. Future experimental studies have to reveal the mechanisms which the "anomalous" AP initiation in cortical neurons is based on. An important step in this direction is to characterize the dynamics of voltage-gated sodium channels in a physiological relevant regime and to assess up to which degree processes such as phosphorylation (Cantrell \& Catterall, 2001) or other second messenger pathways have an impact on the gating kinetics of voltage-gated channels and consequently on the dynamics of single neurons.

Yet, as large scale modeling of neuronal networks is becoming more and more feasible, there is an urgent need for the identification of simple neuron models which realistically reproduce the dynamical features of cortical neurons. An essential step in this direction is to systematically derive phenomenological neuron models which can reproduce the subthreshold dynamics and the AP generation of real neurons. This issue was first addressed in a study by Fourcaud-Trocmé et al. (2003). The authors of this study developed a phenomenological neuron model, the EIF model, which could reproduce the stationary and dynamical linear response properties of a high-dimensional conductance-based neuron model. In this thesis, I used a similar approach by studying the dynamical population response of the generalized $\theta$-neuron. However, I did not attempt to reproduce the dynamics of conductance-based models, because, as I showed in chapter 4, the dynamics of cortical AP initiation substantially differs from the dynamics of this class of models. Besides a rapid onset, which can be easily accounted for in the generalized $\theta$-neuron, cortical neurons also exhibit a large variability in their AP onsets. The dynamical consequences of this variability were specifically assessed in chapter 5 , using the $V-\psi$ model. Naturally, a straightforward extension would be to combine the generalized $\theta$-neuron and the $V-\psi$ model. The model parameters should then be adjusted such that the resulting response properties match the dynamical response properties of cortical neurons.

A second, alternative approach to construct realistic phenomenological neuron models was established in (Fröhlich et al., 2005): Based on a large number of features constructed from the subthreshold membrane potential fluctuations of cortical neurons in vivo, we derived the subset of features which predicts the occurrence and timing of APs with a high precision, using Support Vector Machine algorithms (Schölkopf \& Smola, 2002). This approach has the advantage that it allows for the systematic construction of models without an a-priori bias.

It is important to keep in mind, however, that cortical neurons are divided into many functional classes, and that even within one class, neurons exhibit a large variability in their intrinsic dynamical properties. Therefore, it can not be expected that it will be possible to derive one neuron model which faithfully describes the dynamics of all neurons in the brain. The functional importance of this heterogeneity is an important issue which should also be assessed in future studies. 
Methodologically, I tried to establish in this thesis a balance between the development and analysis of simple, yet dynamically realistic phenomenological models, and the analysis of recordings of real neurons. I believe that this approach is most fruitful to uncover and understand the dynamical mechanisms that are implemented in the cortex and which enable the brain to accomplish complex sensory integrations and higher computations. This approach has to be contrasted with a modeling approach in which as much detail as possible is put together, by e.g. constructing spatially distributed neuron models which are subsequently interconnected in a complicated fashion. From what I learned during my thesis, it is too easy in such an approach to oversee essential features which may be crucial for a realistic modeling of neuronal computation.

Taken together, I am confident that the results presented in this thesis are a small step towards the understanding of the dynamics of cortical processing and, consequently, towards the long term goal to understand the functioning of the brain. 


\section{Bibliography}

Abbott, L. (1999). Lapicque's introduction of the integrate-and-fire model neuron (1907). Brain Res Bull, 50, 303-304.

Abramowitz, M. \& Stegun, I. (1972). Tables of Mathematical Functions. (New York: Dover Publications).

Anderson, J., Lampl, I., Gillespie, D., \& Ferster, D. (2000). The contribution of noise to contrast invariance of orientation tuning in cat visual cortex. Science, 290, 1968-1972.

Arieli, A., Sterkin, A., Grinvald, A., \& Aertsen, A. (1996). Dynamics of ongoing activity: explanation of the large variability in evoked cortical responses. Science, 273, 1868-1871.

Attwell, D. \& Laughlin, S. (2001). An energy budget for signaling in the grey matter of the brain. J Cereb Blood Flow Metab, 21, 1133-1145.

Azouz, R. \& Gray, C. (2000). Dynamic spike threshold reveals a mechanism for synaptic coincidence detection in cortical neurons in vivo. Proc Natl Acad Sci U S A, 97, 8110-8115.

Azouz, R. \& Gray, C. (2003). Adaptive coincidence detection and dynamic gain control in visual cortical neurons in vivo. Neuron, 37, 513-523.

Benda, J. \& Herz, A. (2003). A universal model for spike-frequency adaptation. Neural Comput, 15, $2523-2564$.

Bernander, O., Douglas, R., Martin, K., \& Koch, C. (1991). Synaptic background activity influences spatiotemporal integration in single pyramidal cells. Proc Natl Acad Sci U S A, 88, 11569-11573.

Bernstein, J. (1868). Über den zeitlichen Verlauf der negativen Schwankungen des Nervenstroms. Pflügers Archiv, 1, 173-207.

Braitenberg, V. \& Schüz, A. (1998). Cortex: Statistics and Geometry of Neuronal Connectivity. 2nd thoroughly revised edition of: Anatomy of the Cortex. Statistics and Geometry (1991). (Springer Verlag).

Brumberg, J. (2002). Firing pattern modulation by oscillatory input in supragranular pyramidal neurons. Neuroscience, 114, 239-246.

Brunel, N. (2000). Dynamics of sparsely connected networks of excitatory and inhibitory spiking neurons. J Comput Neurosci, 8, 183-208.

Brunel, N., Chance, F., Fourcaud, N., \& Abbott, L. (2001). Effects of synaptic noise and filtering on the frequency response of spiking neurons. Phys Rev Lett, 86, 2186-2189. 
Brunel, N. \& Hakim, V. (1999). Fast global oscillations in networks of integrate-and-fire neurons with low firing rates. Neural Comput, 11, 1621-1671.

Brunel, N. \& Latham, P. (2003). Firing rate of the noisy quadratic integrate-and-fire neuron. Neural Comput, 15, 2281-2306.

Brunel, N. \& Wang, X. (2001). Effects of neuromodulation in a cortical network model of object working memory dominated by recurrent inhibition. J Comput Neurosci, 11, 63-85.

Burkitt, A. (2001). Balanced neurons: analysis of leaky integrate-and-fire neurons with reversal potentials. Biol Cybern, 85, 247-255.

Calvin, W. \& Stevens, C. (1967). Synaptic noise as a source of variability in the interval between action potentials. Science, $155,842-844$.

Calvin, W. \& Stevens, C. (1968). Synaptic noise and other sources of randomness in motoneuron interspike intervals. J Neurophysiol, 31, 574-587.

Cantrell, A. \& Catterall, W. (2001). Neuromodulation of Na+ channels: an unexpected form of cellular plasticity. Nat Rev Neurosci, 2, 397-407.

Chacron, M., Pakdaman, K., \& Longtin, A. (2003). Interspike interval correlations, memory, adaptation, and refractoriness in a leaky integrate-and-fire model with threshold fatigue. Neural Comput, $15,253-278$.

Chance, F., Abbott, L., \& Reyes, A. (2002). Gain modulation from background synaptic input. Neuron, 35, 773-782.

Chiu, C. \& Weliky, M. (2001). Spontaneous activity in developing ferret visual cortex in vivo. J Neurosci, 21, 8906-8914.

Chiu, S. (1980). Asymmetry currents in the mammalian myelinated nerve. J Physiol, 309, 499-519.

Clark, B., Monsivais, P., Branco, T., London, M., \& Häusser, M. (2005). The site of action potential initiation in cerebellar Purkinje neurons. Nat Neurosci, 8, 137-139.

Coddington, E. \& Levinson, N. (1955). Theory of ordinary differential equations. (New York: McGraw-Hill).

Colbert, C. \& Johnston, D. (1996). Axonal action-potential initiation and Na+ channel densities in the soma and axon initial segment of subicular pyramidal neurons. J Neurosci, 16, 6676-6686.

Colbert, C. \& Pan, E. (2002). Ion channel properties underlying axonal action potential initiation in pyramidal neurons. Nat Neurosci, 5, 533-538.

Colet, P., San Miguel, M., Casademunt, J., \& Sancho, J. (1989). Relaxation from a marginal state in optical bistability. Phys Rev A, 39, 149-156.

Creutzfeldt, O. (1983). Cortex Cerebri. (Berlin: Springer).

Denker, M., Timme, M., Diesmann, M., Wolf, F., \& Geisel, T. (2004). Breaking synchrony by heterogeneity in complex networks. Phys Rev Lett, 92, 074103-074103. 
Destexhe, A. \& Paré, D. (1999). Impact of network activity on the integrative properties of neocortical pyramidal neurons in vivo. J Neurophysiol, 81, 1531-1547.

Destexhe, A., Rudolph, M., Fellous, J., \& Sejnowski, T. (2001). Fluctuating synaptic conductances recreate in vivo-like activity in neocortical neurons. Neuroscience, 107, 13-24.

DeWeese, M., Wehr, M., \& Zador, A. (2003). Binary spiking in auditory cortex. J Neurosci, 23, 7940-7949.

Diesmann, M., Gewaltig, M., \& Aertsen, A. (1999). Stable propagation of synchronous spiking in cortical neural networks. Nature, 402, 529-533.

Engel, A., Fries, P., König, P., Brecht, M., \& Singer, W. (1999). Temporal binding, binocular rivalry, and consciousness. Conscious Cogn, 8, 128-151.

Ermentrout, G. \& Kopell, N. (1986). Parabolic bursting in an excitable system coupled with a slow oscillation. SIAM-J. Appl. Math., 2, 233-253.

Ermentrout, G. \& Rinzel, J. (1984). Beyond a pacemaker's entrainment limit: phase walk-through. Am J Physiol, 246, 102-106.

Fellous, J., Rudolph, M., Destexhe, A., \& Sejnowski, T. (2003). Synaptic background noise controls the input/output characteristics of single cells in an in vitro model of in vivo activity. Neuroscience, $122,811-829$.

Fitzhugh, R. (1960). Thresholds and plateaus in the Hodgkin-Huxley nerve equations. J Gen Physiol, $43,867-896$.

Fitzpatrick, D., Batra, R., Stanford, T., \& Kuwada, S. (1997). A neuronal population code for sound localization. Nature, 388, 871-874.

Fourcaud, N. \& Brunel, N. (2002). Dynamics of the firing probability of noisy integrate-and-fire neurons. Neural Comput, 14, 2057-2110.

Fourcaud-Trocmé, N., Hansel, D., van Vreeswijk, C., \& Brunel, N. (2003). How spike generation mechanisms determine the neuronal response to fluctuating inputs. J Neurosci, 23, 11628-11640.

Fröhlich, H., Naundorf, B., Volgushev, M., \& Wolf, F. (2005). Which features trigger action potentials in cortical neurons in vivo? In International Joint Conference on Neural Networks (IJCNN).

Gardiner, C. (2004). Handbook of Stochastic Methods, 3nd edition. (Berlin: Springer).

Georgopoulos, A., Schwartz, A., \& Kettner, R. (1986). Neuronal population coding of movement direction. Science, 233, 1416-1419.

Gerstein, G. \& Mandelbrot, B. (1964). Random walk models for the spike activity of a single neuron. Biophys J, 71, 41-68.

Gerstner, W. (2000). Population dynamics of spiking neurons: fast transients, asynchronous states, and locking. Neural Comput, 12, 43-89.

Gerstner, W. \& Kistler, W. (2002). Spiking Neuron Models. (Cambridge: Cambridge University Press). 
Grassberger, P. \& Procaccia, I. (1983). Characterization of strange attractors. Phys Rev Lett, 50, 346-349.

Guckenheimer, J. (1975). Isochrons and phaseless sets. J Math Biol, 1, 259-273.

Gutkin, B. \& Ermentrout, G. (1998). Dynamics of membrane excitability determine interspike interval variability: a link between spike generation mechanisms and cortical spike train statistics. Neural Comput, 10, 1047-1065.

Hansel, D. \& Mato, G. (2003). Asynchronous states and the emergence of synchrony in large networks of interacting excitatory and inhibitory neurons. Neural Comput, 15, 1-56.

Hansel, D. \& Sompolinsky, H. (1996). Chaos and synchrony in a model of a hypercolumn in visual cortex. J Comput Neurosci, 3, 7-34.

Häusser, M., Stuart, G., Racca, C., \& Sakmann, B. (1995). Axonal initiation and active dendritic propagation of action potentials in substantia nigra neurons. Neuron, 15, 637-647.

Hebb, D. (1949). The Organization of Behavior; a Neuropsychological Theory. (New York: Wiley).

Henze, D. \& Buzsáki, G. (2001). Action potential threshold of hippocampal pyramidal cells in vivo is increased by recent spiking activity. Neuroscience, 105, 121-130.

Hille, B. (2001). Ion Channels of Excitable Membranes. 3. Edition. (Sunderland, MA: Sinauer).

Hille, B., Woodhull, A., \& Shapiro, B. (1975). Negative surface charge near sodium channels of nerve: divalent ions, monovalent ions, and pH. Philos Trans R Soc Lond B Biol Sci, 270, 301-318.

Hodgkin, A. \& Huxley, A. (1952). A quantitative description of membrane current and its application to conduction and excitation in nerve. J Physiol, 117, 500-544.

Huguenard, J., Hamill, O., \& Prince, D. (1988). Developmental changes in Na+ conductances in rat neocortical neurons: appearance of a slowly inactivating component. J Neurophysiol, 59, 778-795.

Huguenard, J., Hamill, O., \& Prince, D. (1989). Sodium channels in dendrites of rat cortical pyramidal neurons. Proc Natl Acad Sci U S A, 86, 2473-2477.

Huxley, F. (1963). The quantitative analysis of excitation and conduction in nerve. In Nobel Lectures, Physiology or Medicine 1963-1970. (Elsevier Publishing Company, Amsterdam).

Iwasa, K., Ehrenstein, G., Moran, N., \& Jia, M. (1986). Evidence for interactions between batrachotoxin-modified channels in hybrid neuroblastoma cells. Biophys J, 50, 531-537.

Jacobson, G., Diba, K., Yaron-Jakoubovitch, A., Oz, Y., Koch, C., Segev, I., \& Yarom, Y. (2005). Subthreshold voltage noise of rat neocortical pyramidal neurones. J Physiol, 564, 145-160.

Jolivet, R., Lewis, T., \& Gerstner, W. (2004). Generalized integrate-and-fire models of neuronal activity approximate spike trains of a detailed model to a high degree of accuracy. J Neurophysiol, 92, 959-976.

Kantz, H. \& Schreiber, T. (1997). Nonlinear time series analysis. (Cambridge: Cambridge University Press). 
Knight, B. (1972). Dynamics of encoding in a population of neurons. J Gen Physiol, 59, 734-766.

Knight, B. (2000). Dynamics of encoding in neuron populations: some general mathematical features. Neural Comput, 12, 473-518.

Knight, B., Omurtag, A., \& Sirovich, L. (2000). The approach of a neuron population firing rate to a new equilibrium: an exact theoretical result. Neural Comput, 12, 1045-1055.

Koch, C., Bernander, O., \& Douglas, R. (1995). Do neurons have a voltage or a current threshold for action potential initiation? J Comput Neurosci, 2, 63-82.

König, P., Engel, A., \& Singer, W. (1996). Integrator or coincidence detector? The role of the cortical neuron revisited. Trends Neurosci, 19, 130-137.

Lapicque, L. (1907). Recherches qantitatives sur l'excitation electrique des nerfs traitée comme une polarization. J Physiol Pathol Gen, 9, 620-635.

Laughlin, S. \& Sejnowski, T. (2003). Communication in neuronal networks. Science, 301, 18701874.

Lee, C., Rohrer, W., \& Sparks, D. (1988). Population coding of saccadic eye movements by neurons in the superior colliculus. Nature, 332, 357-360.

Lehoucq, R., Sorensen, D., \& Yang, C. (1998). Arpack User's Guide: Solution of Large-Scale Eigenvalue Problems With Implicitly Restarted Arnoldi Methods. (Philadelphia: SIAM).

Lennie, P. (2003). The cost of cortical computation. Curr Biol, 13, 493-497.

Lindner, B. \& Longtin, A. (2005). Effect of an exponentially decaying threshold on the firing statistics of a stochastic integrate-and-fire neuron. J Theor Biol, 232, 505-521.

Lindner, B., Longtin, A., \& Bulsara, A. (2003). Analytic expressions for rate and CV of a type I neuron driven by white gaussian noise. Neural Comput, 15, 1760-1787.

Lindner, B. \& Schimansky-Geier, L. (2001). Transmission of noise coded versus additive signals through a neuronal ensemble. Phys Rev Lett, 86, 2934-2937.

Liu, Y. \& Dilger, J. (1993). Application of the one- and two-dimensional Ising models to studies of cooperativity between ion channels. Biophys J, 64, 26-35.

Lübke, J., Markram, H., Frotscher, M., \& Sakmann, B. (1996). Frequency and dendritic distribution of autapses established by layer 5 pyramidal neurons in the developing rat neocortex: comparison with synaptic innervation of adjacent neurons of the same class. J Neurosci, 16, 3209-3218.

MacKinnon, R. (1991). Determination of the subunit stoichiometry of a voltage-activated potassium channel. Nature, 350, 232-235.

Mainen, Z. \& Sejnowski, T. (1995). Reliability of spike timing in neocortical neurons. Science, 268, $1503-1506$.

Margrie, T., Brecht, M., \& Sakmann, B. (2002). In vivo, low-resistance, whole-cell recordings from neurons in the anaesthetized and awake mammalian brain. Pflügers Arch, 444, 491-498. 
Martina, M. \& Jonas, P. (1997). Functional differences in Na+ channel gating between fast-spiking interneurones and principal neurones of rat hippocampus. J Physiol, 505, 593-603.

Marx, S., Ondrias, K., \& Marks, A. (1998). Coupled gating between individual skeletal muscle Ca2+ release channels (ryanodine receptors). Science, 281, 818-821.

Mattia, M. \& Del Giudice, P. (2002). Population dynamics of interacting spiking neurons. Phys Rev E Stat Nonlin Soft Matter Phys, 66, 051917-051917.

Mazurek, M. \& Shadlen, M. (2002). Limits to the temporal fidelity of cortical spike rate signals. Nat Neurosci, 5, 463-471.

McCulloch, W. \& Pitts, W. (1943). A logical calculus of ideas immanent in nervous activity. Bull Math Biophys, 5, 115-133.

Morris, C. \& Lecar, H. (1981). Voltage oscillations in the barnacle giant muscle fiber. Biophys J, 35, 193-213.

Naundorf, B., Geisel, T., \& Wolf, F. (2003). The Intrinsic Time Scale of Transient Neuronal Responses. xxx.lanl.gov, physics/0307135.

Naundorf, B., Geisel, T., \& Wolf, F. (2005a). Action potential onset dynamics and the response speed of neuronal populations. J Comput Neurosci, 18, 297-309.

Naundorf, B., Geisel, T., \& Wolf, F. (2005b). Dynamical Response Properties of a Canonical Model of Type-I Membranes. Neurocomputing, 65, 421-428.

Naundorf, B., Wolf, F., \& Volgushev, M. (2005c). Unique features of action potential initiation in cortical neurons. Submitted to Nature, Under review.

Neher, E., Sakmann, B., \& Steinbach, J. (1978). The extracellular patch clamp: a method for resolving currents through individual open channels in biological membranes. Pflügers Arch, 375, 219-228.

Nernst, W. (1888). Zur Kinetik der in Lösung befindlichen Körper. Erste Abhandlung. Theorie der Diffusion. Z Phys Chem, 2, 613-637.

Neumcke, B. \& Stämpfli, R. (1982). Sodium currents and sodium-current fluctuations in rat myelinated nerve fibres. J Physiol, 329, 163-184.

Nowak, L., Sanchez-Vives, M., \& McCormick, D. (1997). Influence of low and high frequency inputs on spike timing in visual cortical neurons. Cereb Cortex, 7, 487-501.

O' Kusky, J. \& Colonnier, M. (1982). A laminar analysis of the number of neurons, glia, and synapses in the adult cortex (area 17) of adult macaque monkeys. J Comp Neurol, 210, 278-290.

Paré, D., Lang, E., \& Destexhe, A. (1998). Inhibitory control of somatodendritic interactions underlying action potentials in neocortical pyramidal neurons in vivo: an intracellular and computational study. Neuroscience, $84,377-402$.

Patlak, J. (1991). Molecular kinetics of voltage-dependent Na+ channels. Physiol Rev, 71, 1047-1080.

Pei, X., Volgushev, M., Vidyasagar, T., \& Creutzfeldt, O. (1991). Whole cell recording and conductance measurements in cat visual cortex in-vivo. Neuroreport, 2, 485-488. 
Poirazi, P., Brannon, T., \& Mel, B. (2003). Pyramidal neuron as two-layer neural network. Neuron, 37, 989-999.

Polsky, A., Mel, B., \& Schiller, J. (2004). Computational subunits in thin dendrites of pyramidal cells. Nat Neurosci, 7, 621-627.

Populin, L. \& Yin, T. (1998). Behavioral studies of sound localization in the cat. J Neurosci, 18, $2147-2160$.

Prut, Y., Vaadia, E., Bergman, H., Haalman, I., Slovin, H., \& Abeles, M. (1998). Spatiotemporal structure of cortical activity: properties and behavioral relevance. J Neurophysiol, 79, 2857-2874.

Ramon y Cajal, S. (1906). The structure and connexions of neurons. In Nobel Lectures, Physiology or Medicine 1901-1921. (Elsevier Publishing Company, Amsterdam).

Rasband, M. \& Shrager, P. (2000). Ion channel sequestration in central nervous system axons. J Physiol, 525, 63-73.

Reichl, L. (1988). Transitions in the Floquet Rates of a Driven Stochastic System. J. Stat. Phys., 53, 41.

Reichl, L. (1998). A Modern Course in Statistical Physics. 2. Edition. (Berlin: Wiley).

Riehle, A., Grün, S., Diesmann, M., \& Aertsen, A. (1997). Spike synchronization and rate modulation differentially involved in motor cortical function. Science, 278, 1950-1953.

Rinzel, J. \& Ermentrout, G. (1998). Analysis of neural excitability and oscillations. In Methods in Neuronal Modeling: From Ions to Networks. Second Edition, C. Koch \& I. Segev, eds. (Mit Press, Cambridge).

Risken, H. (1996). The Fokker Planck Equation: Methods of Solution and Applications. (Berlin: Springer).

Rudolph, M. \& Destexhe, A. (2003). The discharge variability of neocortical neurons during highconductance states. Neuroscience, 119, 855-873.

Sanchez, J., Dani, J., Siemen, D., \& Hille, B. (1986). Slow permeation of organic cations in acetylcholine receptor channels. J Gen Physiol, 87, 985-1001.

Schneidman, E., Freedman, B., \& Segev, I. (1998). Ion channel stochasticity may be critical in determining the reliability and precision of spike timing. Neural Comput, 10, 1679-1703.

Schölkopf, B. \& Smola, A. J. (2002). Learning with Kernels. (Cambridge, MA: MIT Press).

Shadlen, M. \& Newsome, W. (1998). The variable discharge of cortical neurons: implications for connectivity, computation, and information coding. J Neurosci, 18, 3870-3896.

Shamir, M. \& Sompolinsky, H. (2004). Nonlinear population codes. Neural Comput, 16, 1105-1136.

Sigworth, F. (1980). The conductance of sodium channels under conditions of reduced current at the node of Ranvier. J Physiol, 307, 131-142.

Sigworth, F. (1981). Covariance of nonstationary sodium current fluctuations at the node of Ranvier. Biophys J, 34, 111-133. 
Silberberg, G., Bethge, M., Markram, H., Pawelzik, K., \& Tsodyks, M. (2004). Dynamics of population rate codes in ensembles of neocortical neurons. J Neurophysiol, 91, 704-709.

Softky, W. \& Koch, C. (1993). The highly irregular firing of cortical cells is inconsistent with temporal integration of random EPSPs. J Neurosci, 13, 334-350.

Steinmetz, P., Manwani, A., Koch, C., London, M., \& Segev, I. (2000). Subthreshold voltage noise due to channel fluctuations in active neuronal membranes. J Comput Neurosci, 9, 133-148.

Stratonovich, R. (1963). Topics in the Theory of Random Noise, Vols. I and II. (New York: Gordon and Breach).

Strogatz, S. (2001). Nonlinear Dynamics and Chaos: With Applications to Physics, Biology, Chemistry and Engineering. (Philadelphia: Perseus Books Group).

Stuart, G., Schiller, J., \& Sakmann, B. (1997). Action potential initiation and propagation in rat neocortical pyramidal neurons. J Physiol, 505, 617-632.

Takens, F. (1981). Dynamical Systems and Turbulence. (New York: Springer Lecture Notes in Mathematics. Springer).

Tateno, T., Harsch, A., \& Robinson, H. (2004). Threshold firing frequency-current relationships of neurons in rat somatosensory cortex: type 1 and type 2 dynamics. J Neurophysiol, 92, 2283-2294.

Tiesinga, P., José, J., \& Sejnowski, T. (2000). Comparison of current-driven and conductance-driven neocortical model neurons with Hodgkin-Huxley voltage-gated channels. Phys Rev E Stat Phys Plasmas Fluids Relat Interdiscip Topics, 62, 8413-8419.

Tiesinga, P., Thomas, P., Fellous, J.-M., \& Sejnowski, T. (2002). Reliability, precision and the neuronal code. In Society for Neuroscience Abstracts 2002, Orlando.

Trefethen, N. \& Bau, D. (1997). Numerical Linear Algebra. (Philadelphia: SIAM).

Tsodyks, M. \& Sejnowski, W. (1995). Rapid state switching in balanced cortical network models. Network-Computation in Neural Systems, 6, 111-124.

Tuckwell, H. (1978). Recurrent inhibition and afterhyperpolarization: effects on neuronal discharge. Biol Cybern, 30, 115-123.

Tuckwell, H., Wan, F., \& Rospars, J. (2002). A spatial stochastic neuronal model with OrnsteinUhlenbeck input current. Biol Cybern, 86, 137-145.

Uhlenbeck, G. \& Ornstein, L. (1930). On the theory of brownian motion. Phys. Rev., 36, 823-841.

van Kampen, N. (1981). Ito versus Stratonovich. J Stat Phys, 24, 175-187.

van Vreeswijk, C. \& Sompolinsky, H. (1996). Chaos in neuronal networks with balanced excitatory and inhibitory activity. Science, 274, 1724-1726.

van Vreeswijk, C. \& Sompolinsky, H. (1998). Chaotic balanced state in a model of cortical circuits. Neural Comput, 10, 1321-1371.

VanRullen, R. \& Thorpe, S. (2001). Is it a bird? Is it a plane? Ultra-rapid visual categorisation of natural and artifactual objects. Perception, 30, 655-668. 
Vasudevan, R. \& Vittal, P. (1982). Time-dependent barriers and first-passage times-different types of neuronal models. Neurol Res, 4, 63-87.

Veenstra, R., Wang, H., Beyer, E., \& Brink, P. (1994). Selective dye and ionic permeability of gap junction channels formed by connexin45. Circ Res, 75, 483-490.

Volgushev, M. \& Eysel, U. (2000). Noise makes sense in neuronal computing. Science, 290, 19081909.

Volgushev, M., Pernberg, J., \& Eysel, U. (2002). A novel mechanism of response selectivity of neurons in cat visual cortex. J Physiol, 540, 307-320.

Volgushev, M., Vidyasagar, T., Chistiakova, M., Yousef, T., \& Eysel, U. (2000). Membrane properties and spike generation in rat visual cortical cells during reversible cooling. J Physiol, 522, 59-76.

Wang, X., Liu, Y., Sanchez-Vives, M., \& McCormick, D. (2003). Adaptation and temporal decorrelation by single neurons in the primary visual cortex. J Neurophysiol, 89, 3279-3293.

White, J., Rubinstein, J., \& Kay, A. (2000). Channel noise in neurons. Trends Neurosci, 23, 131-137.

Wilbur, W. \& Rinzel, J. (1983). A theoretical basis for large coefficient of variation and bimodality in neuronal interspike interval distributions. J Theor Biol, 105, 345-368.

Winfree, A. (1967). Biological rhythms and the behavior of populations of coupled oscillators. J Theor Biol, 16, 15-42.

Winfree, A. (2001). The geometry of biological time. Second Edition. (New York: Springer).

Wolf, F. \& Geisel, T. (1998). Spontaneous pinwheel annihilation during visual development. Nature, $395,73-78$.

Woodhull, A. (1973). Ionic blockage of sodium channels in nerve. J Gen Physiol, 61, 687-708.

Yeramian, E., Trautmann, A., \& Claverie, P. (1986). Acetylcholine receptors are not functionally independent. Biophys J, 50, 253-263.

Young, M. \& Yamane, S. (1992). Sparse population coding of faces in the inferotemporal cortex. Science, 256, 1327-1331. 


\section{Acknowledgments}

Many people contributed to the successful creation of this thesis.

First of all, I would like to thank Prof. Theo Geisel for re-accepting me in his group. It is due to him that I had the opportunity to work in a group with a unique atmosphere of friendliness and excellent working conditions. He did not only teach me fundamental skiing techniques, but also fully supported me in developing myself scientifically. Thank you Theo!

This thesis would not have been possible in this form without the constant and creative support of Dr. Fred Wolf. Fred not only introduced me into the field of theoretical neuroscience, but also taught me how to write papers and fight for their publication, how to give talks and how to work scientifically. He constantly encouraged me, to broaden my view on different scientific topics and, at the same time, helped me to always focus on the important questions. Thank you very much, Fred!

During the last three years, I collaborated with different people, some of them made their way into this thesis, some didn't. I am particularly indebted to Dr. Maxim Volgushev who generously provided me with his experimental data. The discussions with him greatly expanded my knowledge and helped me to shape my view on experimental neuroscience.

I would like to thank Holger Fröhlich, with who I not only worked together to explore the world of Support Vector machines, but who is even more a good friend and a person you can spend the complete night discussing life and similarly involved topics.

For all administrative support, I would like to thank Corinna Trautsch, Regina Wunderlich and Katharina Jeremias.

For setting up and doing a great job in administrating the computer cluster, I would like to thank Yorck-Fabian Beensen, Wolf-Dieter Brandt, Ragnar Fleischmann, Denny Fliegner, Gerhard Nolte.

I would like to thank Vitaly Belik, Oliver Bendix, Dirk Brockmann, Kai Bröking, Vincent David, Michael Denker, Silke Dodel, Markus Diesmann, Ragnar Fleischmann, Sven Goedeke, Joachim Hass, Dominik Heide, Holger Hennig, Michael Herrmann, Moritz Hiller, Min Huang, Lars Hufnagel, Sven Jahnke, Matthias Kaschube, Christoph Kirst, Marten Kopp, Tsampikos Kottos, Anna Levina, Antonio Mendez-Bermudez, Raoul Memmesheimer, Abigail Morrison, Alexander Ossipov, Manamohan Prusty, Mathias Puhlmann, Lars Reichl, Holger Schanz, Hendrik (Hecke) Schrobsdorff, Michael Schnabel and Tom Tetzlaff. All of you contributed in your own way to the great working atmosphere which I experienced during the last three years.

Special thanks go to Marc Timme with who I published my first scientific paper.

I am greatly indebted to my girlfriend Eleanor Livesey. Elli gave me constant personal support, proof read the thesis and accepted all my personal ups and downs with great understanding and love Thank you so much, Elli!

Last but not least, I would like to thank my parents for their constant support during the past years. 